\title{
Can Sustainability Standards Promote Socioeconomic Development in the Small Farm Sector? Insights from Coffee Producers in Uganda
}

\author{
Dissertation \\ to obtain the doctoral degree
}

in the International Ph. D. Program for Agricultural Sciences in Goettingen (IPAG) at the Faculty of Agricultural Sciences,

Georg-August-University Goettingen, Germany

presented by

Eva-Marie Meemken

born in Lemgo, Germany

Goettingen, March 2017 
D7

Name of supervisor: Prof. Dr. Matin Qaim

Name of co-supervisor: Prof. Dr. Stephan von Cramon-Taubadel

Name of co-supervisor: Prof. Stephan Klasen, Ph.D.

Date of dissertation: 18.05.2017 


\section{Summary}

Sustainable food production and consumption are important goals for an increasing number of consumers, policy makers, and other actors along global food chains. This is also reflected in the $12^{\text {th }}$ United Nations' Sustainable Development Goal, which emphasizes the need for sustainable consumption and production patterns. As part of this trend, sustainability standards and related labeling of consumer products have gained in importance. Sustainability standards for food products address environmental, social welfare, and human rights issues - and often focus on tropical export crops produced by smallholder farmers in developing countries. Do poor farmers benefit from the boom in sustainability standards? A growing body of literature has analyzed this question, with mixed results. This dissertation is composed of three papers, contributing to this literature in different ways. All three papers are based on a farm household survey conducted in 2015 with smallholder coffee producers in Uganda. In total, we interviewed 455 farm households. Most of these households were already interviewed in 2012, allowing us to use panel data methods to address some of our research questions. Among the sample households, some are certified under Fairtrade, Organic, or UTZ. Other households are not certified under any standard. In each household, we interviewed male and female household members, to the extent possible. This allows us to analyze household-level and individual-level issues and effects.

The number of impact studies on sustainability standards and their effects on farm households in developing countries is growing. Yet, results are mixed and the evidence is not conclusive. We identified several research gaps, which are addressed in the papers presented in this dissertation. The existing work has primarily analyzed effects in terms of purely economic indicators, such as prices or incomes. Welfare outcomes such as household nutrition, child education, and gender equality have received very little scientific attention. Further, most existing studies have looked at the effect of one standard in one country, limiting our understanding of dissimilar effects of different types of standards. Further, almost all available studies are based on cross-sectional data, so that issues of possible selection bias are more difficult to address. To our knowledge, all available, quantitative studies are based on household-level data, so that issues of intra-household distribution of costs and benefits cannot be analyzed. Finally, farmers' preferences have hardly received attention in the literature.

The first paper analyzes and compares effects of Fairtrade and Organic standards on consumption expenditures, child education, and household nutrition, contributing to a 
better understanding of the dissimilar welfare effects of different standards. The analysis is based on panel data, using both survey rounds: 2012 and 2015. In our panel data econometric models (i.e. fixed effects and random effects models) Organic and Fairtrade are included as binary, time-variant treatment variables. We find that both Organic and Fairtrade have positive effects on aggregate living standards. However, notable differences are observed with respect to nutrition and education. Organic contributes to improved nutrition but has no effect on education. For Fairtrade it is exactly the other way around. We explore the mechanisms behind these differences. We conclude that sustainability standards can have positive welfare effects for farm households, which is consistent with most of the existing evidence. Yet, our results also highlight that different standards can have dissimilar effects on various areas of household welfare. Understanding such differences between standards is not possible when considering one aggregate measure of living standard alone. Diverse development objectives require impact analyses with more disaggregated social welfare measures, as used in this study.

This second paper is the first quantitative study to explore heterogeneous treatment effects on women and men. We focus on two standards that explicitly address gender issues, namely Fairtrade and UTZ. Fairtrade and UTZ certified farmer organizations have to comply with non-discrimination policies. Further, they are encouraged to organize workshops on gender equality; to implement special programs tailored to women farmers' needs; and to promote women's participation in regular trainings. Our research objective is to assess if Fairtrade and UTZ benefit individuals in male-headed households (i.e. male household heads and female spouses) and in female-headed households (i.e. female household heads). Our analysis is based on cross-sectional, gender-disaggregated data from our sample of Ugandan coffee producers. For the statistical analysis, we use entropy balancing, allowing us to control for observed heterogeneity between certified and non-certified farmers. To reduce possible bias from unobserved heterogeneity, we use estimates of farmers' willingness to accept (WTA) sustainability standards as a conditioning variable in reweighting the data. We analyze the effect of UTZ and Fairtrade standards on various outcome variables that capture different areas of empowerment. These include gendered asset ownership, participation in trainings and farmer group meetings, access to financial services, and time allocation. We find that sustainability standards increase household assets - including women's assets. In male-headed households, standards also affect the distribution of wealth: While in non-certified households most assets are owned by the male household head alone, in certified households most assets are owned jointly by the male head and female spouse. Certified farmers also have better access to agricultural extension, 
irrespective of their gender. Yet, sustainability standards have no effect on women's access to financial services. We conclude that sustainability standards may not completely eliminate gender disparities, but can at least contribute towards this goal.

The third paper analyzes farmers' preferences for sustainability standards. Standards that are better tailored to farmers' needs and preferences may be more attractive and feasible for a larger number of farmers, thus resulting in higher adoption rates. In this study, we specifically look at gender differences in preferences. A gender focus is important, because coffee and other certified export crops are often controlled by men. Our analysis is based on a choice experiment, which was conducted separately with male and female members of farm households. This choice experiment was included as part of the 2015 survey with Ugandan coffee producers. Attributes included in the choice experiment are: (1) the price received for the coffee sold, (2) agricultural training, (3) gender policies, (4) quality requirements, (5) handling of chemical pesticides, and (6) record keeping. For the statistical analysis, we use mixed logit models. Results indicate that farmers have positive attitudes towards sustainability standards in general. While they dislike bans of productivity-enhancing inputs, agricultural trainings are appreciated. Farmers also prefer standards that encourage better farm management and quality upgrading, suggesting that certification requirements may serve to encourage desirable investments in the small farm sector. Female farmers have a higher preference for sustainability standards than male farmers. Also within households, significant preference heterogeneity between women and men is found. However, we find that both male and female farmers appreciate special female support. In line with the second paper, we conclude that a stronger focus on gender policies could be an interesting option to further explore in some situations.

Based on all three papers, we conclude that sustainability standards can have broader welfare implications, which cannot be captured by only looking at short-term economic effects. Further, effects of - and preferences for - sustainability standards can be heterogeneous, and may vary within households. We also show that different types of standards can have different types of effects.

Generally, our results are in line with many previous studies, suggesting that sustainability standards can be beneficial for farmers who are able to adopt them. However, various studies also draw less positive conclusions. Thus, based on the existing body of literature, sustainability standards should neither be promoted as a silver bullet for poverty reduction, nor should they be seen as an empty promise. 


\section{Acknowledgements}

I thank my supervisor, Prof. Dr. Matin Qaim, for his guidance, helpful feedback, and extraordinary quick responses. Further, I thank Prof. Qaim for being a very good mentor and encouraging me to pursue a career in research. I am looking forward to future collaboration during the next year - and hopefully beyond.

I am also delighted to have Prof. Dr. Stephan von Cramon-Taubadel and Prof. Stephan Klasen, $\mathrm{PhD}$ as members of my thesis committee. Thank you very much for your support!

I thank my coauthors Prakashan Veettil (IRRI) and David Spielman (IFPRI) for hosting me in Delhi and Washington, for fruitful discussions, and their input and support. I also thank Haruna Sekabira for the great collaboration during our field work in Uganda and for helping me to better understand (coffee production in) Uganda. Our survey in Uganda would not have been possible without our committed enumerators, each farmer who participated in our survey, and many other people supporting us. I also thank Brian Chiputwa for sharing the 2012 survey data.

My special thanks also go to my (GlobalFood) friends and colleagues. Being in Goettingen was great - because of you. Thank you for your support, great scientific and non-scientific discussions, and numerous amazing evenings. I especially thank Kerstin Grosch and Malte Ehrich - Goettingen will be less unique to me without you. I also thank my office colleague Dennis Ochieng for enriching discussions about choice experiments.

Finally, I would like to express my deepest gratitude to my parents Ingrid and Dieter Meemken for supporting all my decisions, finally leading me to aim for a $\mathrm{Ph} . \mathrm{D}$.

This research was financially supported by the German Research Foundation (DFG). 


\section{Table of contents}

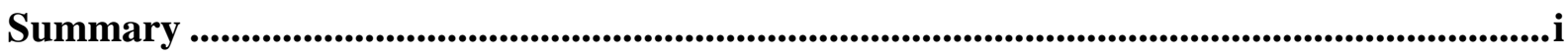

Acknowledgements ..................................................................................................................iv

List of tables ..............................................................................................................................viii

List of figures ..........................................................................................................................................ix

1 General introduction ................................................................................................................... 1

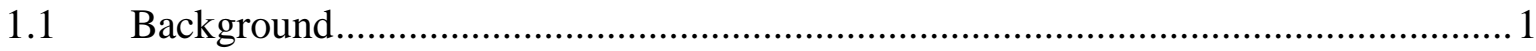

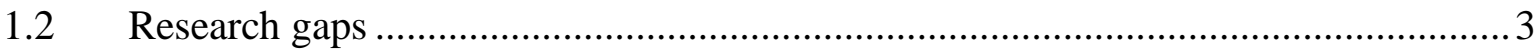

$1.3 \quad$ Research objectives and approach ................................................................

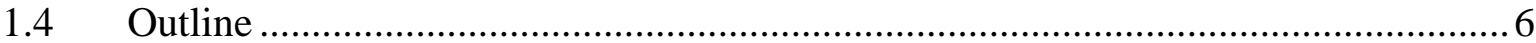

2 Trading off nutrition and education? A panel data analysis of the dissimilar welfare effects of Organic and Fairtrade standards ........................................................... 7

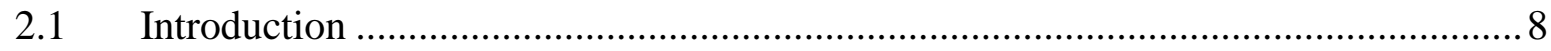

2.2 Sustainability standards in theory and practice ......................................................

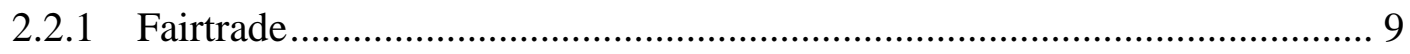

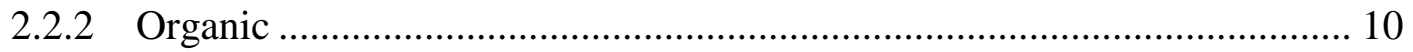

2.2.3 Possible economic impacts ..................................................................... 10

2.2.4 Possible impacts on child education ......................................................... 11

2.2.5 Possible impacts on nutrition................................................................... 12

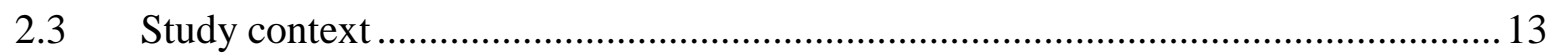

2.3.1 Coffee production in Uganda ………………………………………….... 13

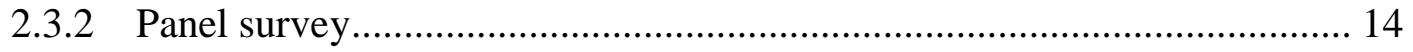

2.3.3 Services of farmer organizations ............................................................. 15

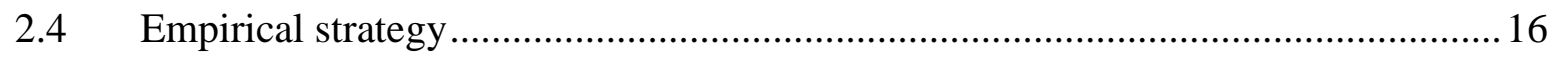

2.4.1 Measurement of outcome variables ........................................................ 16

2.4.2 Panel regression models ............................................................................. 17

2.4.3 Cross-section models........................................................................... 18

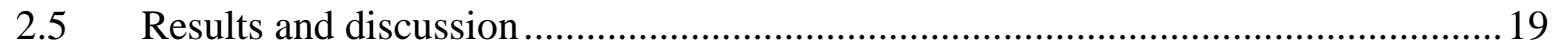

2.5.1 Descriptive statistics ............................................................................. 19

2.5.2 Regression results ............................................................................ 21 
2.5.3 Fairtrade certified households 23

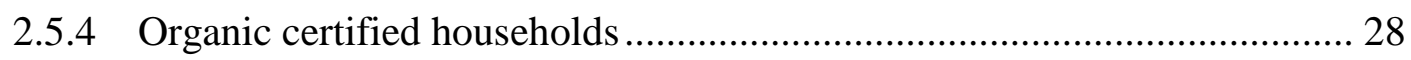

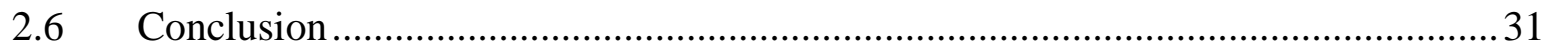

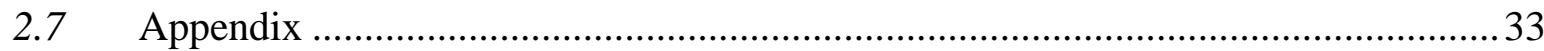

3 Can private food standards promote gender equality in the small farm sector? ......36

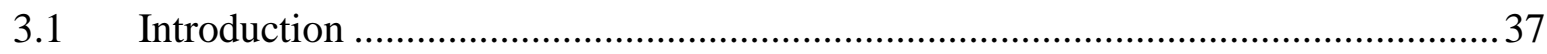

3.2 Sustainability standards, gender components, and possible effects on gender equality

3.2.1 Gender components of Fairtrade and UTZ ............................................. 39

3.2.2 Possible effects on economic empowerment........................................ 40

3.2.3 Possible effects on the division of labor and women's workload ............. 42

3.2.4 Possible effects on social capital ........................................................ 42

3.2.5 Possible effects on the access to services ............................................. 43

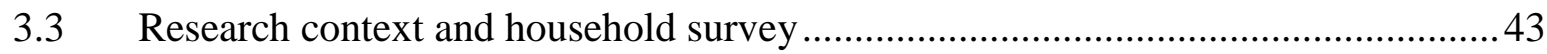

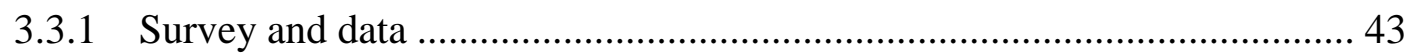

3.3.2 Gender policies implemented by farmer organizations ........................... 45

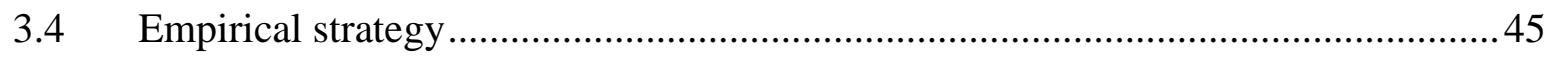

3.4.1 Selection and measurement of outcome variables.................................... 45

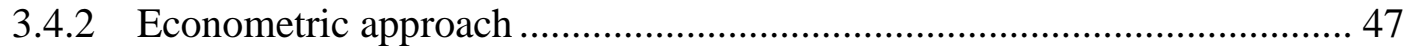

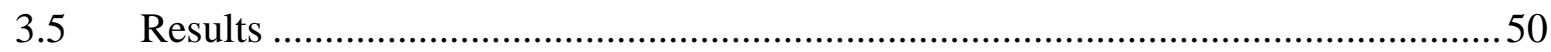

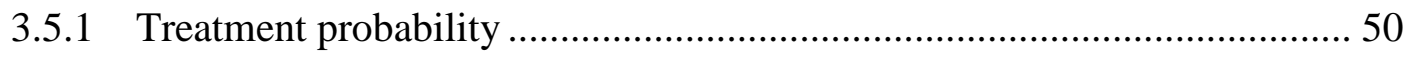

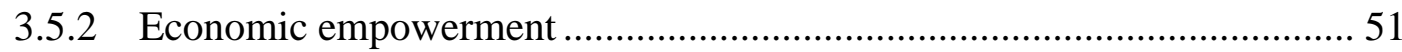

3.5.3 Division of labor within households and workload .................................. 56

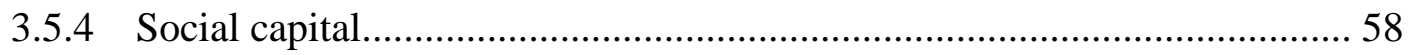

3.5.5 Access to extension and financial services.......................................... 58

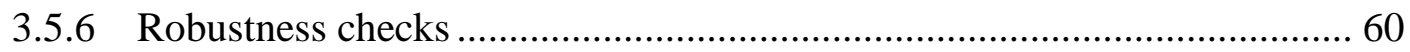

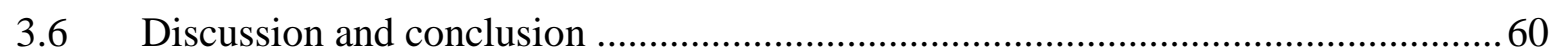

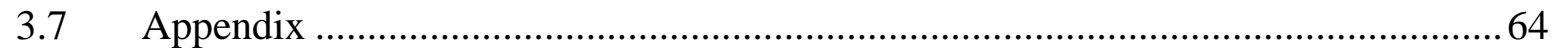

4 Towards improving the design of sustainability standards - A gendered analysis of farmers' preferences.................................................................................................6 67

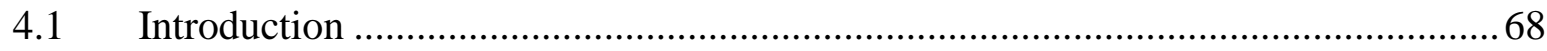




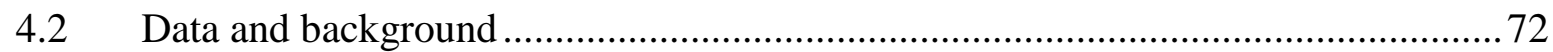

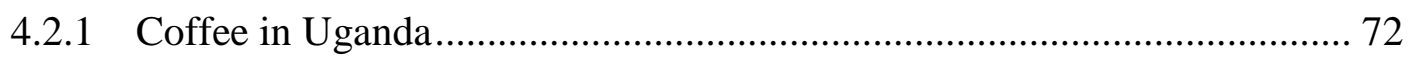

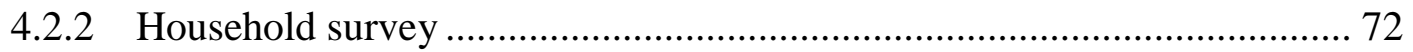

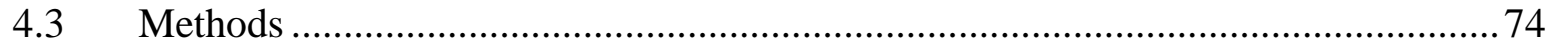

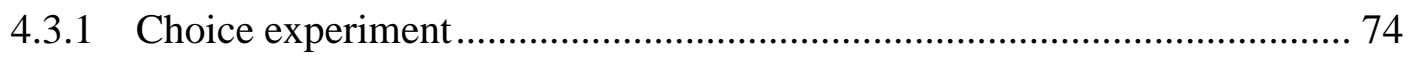

4.3.2 Selection of standard attributes and attribute levels ................................ 75

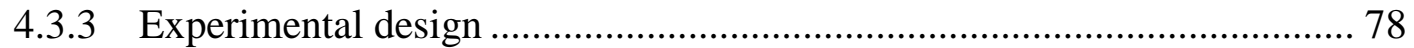

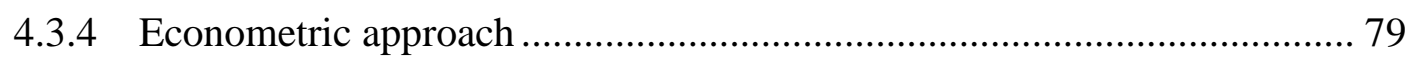

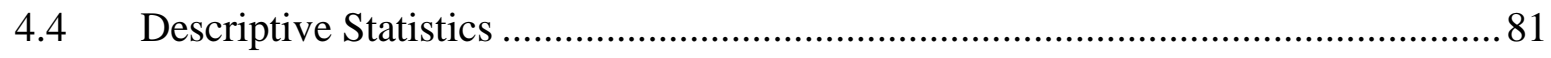

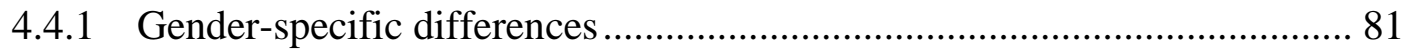

4.4.2 Differences between certified and non-certified households .................... 82

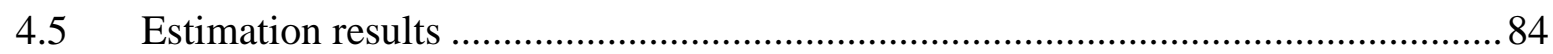

4.5.1 General preferences for sustainability standards .................................. 84

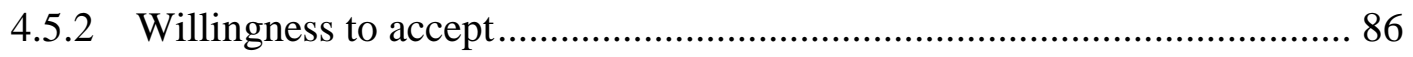

4.5.3 Preference heterogeneity due to previous experience with standards ........ 88

4.5.4 Gender-specific differences in preferences .............................................. 89

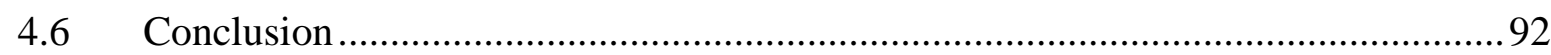

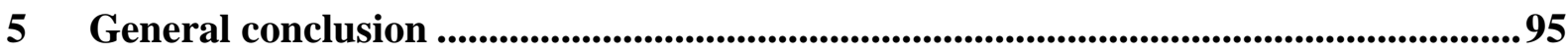

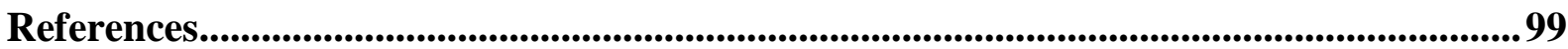

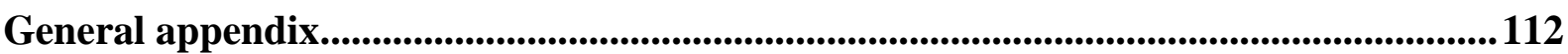




\section{List of tables}

Table 1: Number of sampled households by year and certification status 14

Table 2: Descriptive statistics by certification status (pooled data for 2012 and 2015). 20

Table 3: Effects of certification on household expenditure (panel data models) ........... 22

Table 4: Farming practices by certification status (pooled data for 2012 and 2015) ..... 23

Table 5: Effects of certification on education expenditure (panel data models) ............ 26

Table 6: Effects of certification on individual child education (cross-section data models)

Table 7: Effects of certification on energy and micronutrient deficiency (probit panel data models)

Table A1: Predicated probabilities of participation in Fairtrade and Organic certification

Table A2: Regressions for subsample of non-certified households 34

Table A3: Effects of certification on energy and micronutrient deficiency (linear probability models) 35

Table 8: Overview of sample households and individuals by certification status. 44

Table 9: Treatment probabilities (household level)....................................................... 51

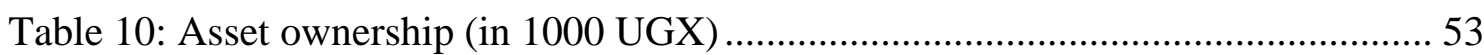

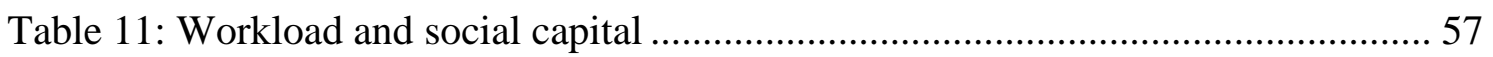

Table 12: Access to extension and financial services.................................................... 59

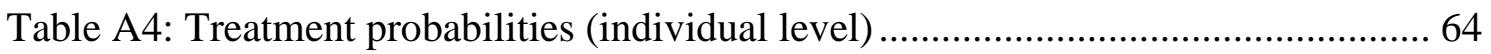

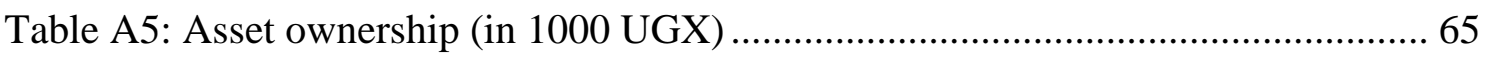

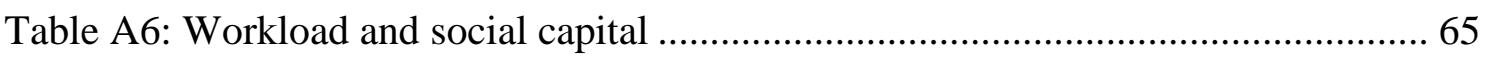

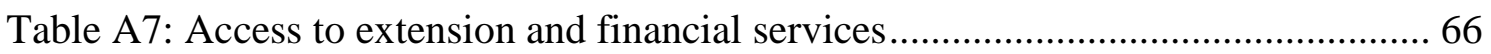

Table 13: Number of sample households by certification status................................. 73

Table 14: Number of individual respondents by gender and decision-making power... 74

Table 15: Overview of attributes and attribute levels used in the choice experiment.... 76

Table 16: Summary statistics by gender (individual level) ....................................... 81

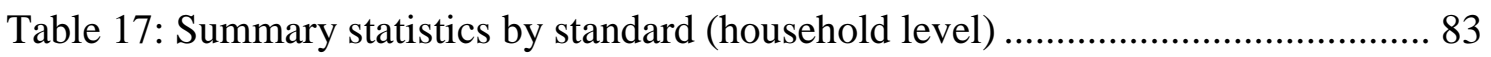

Table 18: Mixed logit model estimates - Base specification and differences by

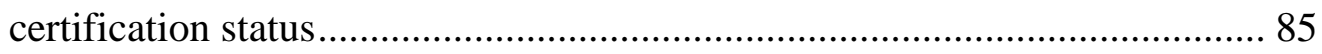

Table 19: Willingness to accept (WTA) estimates (UGX per kilogram of coffee)........ 87

Table 20: Mixed logit model estimates - Specifications to analyze gender differences 90 


\section{List of figures}

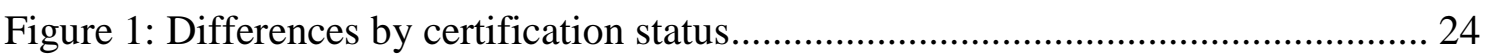

Figure 2: Asset ownership (in 1000 UGX) in male-headed households by category and certification status

Figure 3: Who controls crop revenues in male-headed households? (Percent of

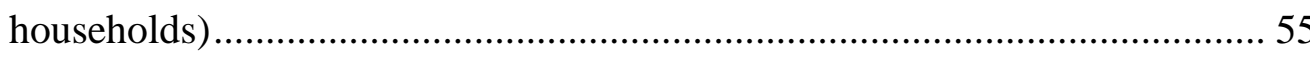

Figure 4: Tasks in coffee production in male-headed households by certification status (percent of households) ............................................................................ 56

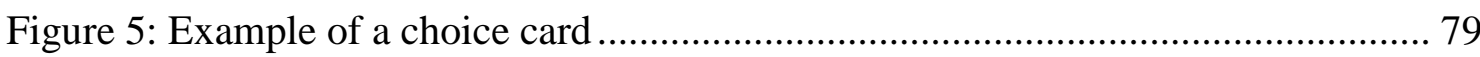




\section{General introduction}

\subsection{Background}

Sustainability standards - such as Fairtrade or Organic - are gaining in importance in global food chains (ITC 2015). These standards are designed to address environmental, social welfare, and human rights issues. Many sustainability standards specifically focus on tropical export crops produced by smallholder farmers and workers in developing countries, such as coffee, cocoa, or tea. Certified coffee, which is predominantly produced by smallholder producer organizations, plays a particularly prominent role in certified markets (Raynolds, Murray, and Heller 2007; ITC 2011, 2015). The area under certified coffee tripled from about one million to about three million hectares between 2008 and 2013. In 2013, an estimated 30 percent of the global coffee area was certified under one of the main certification schemes - 4C Association, Fairtrade, Organic, Rainforest Alliance, and UTZ (ITC 2015). ${ }^{1}$

The proliferation of sustainability standards is attributable to different factors. Sustainable food production and consumption are important goals for an increasing number of consumers, policy makers, and other actors along global food chains. This is also reflected in the $12^{\text {th }}$ United Nations' Sustainable Development Goal, which emphasizes the need for sustainable consumption and production patterns. An increasing number of consumers is willing to pay higher prices for certified products (Hoogland, Boer, and Boersema 2007; Grunert, Hieke, and Wills 2014; Potts et al. 2014). Sustainability certified products are nowadays available in many western supermarkets, facilitating the access to these products and improving their visibility (Elder, Lister, and Dauvergne 2013). Further, development agencies have played a key role in promoting farmer adoption of sustainability standards as a tool for environmental stewardship - and to link farmers to lucrative export markets (Bacon 2005; Handschuch, Wollni, and Villalobos 2013).

Can sustainability standards actually serve to improve the livelihoods of poor farmers and workers while protecting the environment? This question is controversially discussed - and relevant for consumers, development agencies, and other actors along

\footnotetext{
${ }^{1}$ The total area under coffee is about 10 million hectares (FAO 2015). The figures regarding the certified area are an approximation. A major challenge in this estimation is the lack of information on the area that is double certified (e.g. Organic + Fairtrade). See ITC (2015) for further information.
} 
certified value chains. Further, the proliferation of sustainability standards implies that an increasing number of the rural poor are directly or indirectly affected by these standards.

The scientific literature about various aspects of sustainability standards is growing. Generally, the research focus lies on smallholder farmers rather than on workers (Raynolds 2017). This may be because substantially more farmers than workers are certified under standards such as Fairtrade. ${ }^{2}$ One strand of literature uses qualitative data to explore the implications for farmers (e.g. Bacon 2010; Lyon, Bezaury, and Mutersbaugh 2010; Valkila and Nygren 2010; Tallontire, Opondo, and Nelson 2014). While these studies provide important insights, qualitative data do not allow inference about the net welfare effects of standards. Further, results may not be representative, because the samples in many of the qualitative studies are not selected randomly. A second strand of literature uses theoretical approaches to develop hypotheses about price and welfare effects of sustainability standards (e.g. Chambolle and Poret 2013; Yu and Bouamra-Mechemache 2015; Omidvar and Giannakas 2015). Unless tested with empirical data, theoretical models are difficult to verify. A third strand of literature uses household survey data and statistical methods to analyze the effect of standards on farmers (Maertens and Swinnen 2009; Ruben and Fort 2012; Chiputwa, Spielman, and Qaim 2015; van Rijsbergen et al. 2016; Jena, Stellmacher, and Grote 2017). This dissertation contributes to this third strand of literature, as further described below. Impact studies typically focus on one setting and compare a group of certified farmers against a group of non-certified farmers. As all available studies rely on observational data, their key challenge is to deal with farmer self-selection into certification. While earlier studies often failed to address possible issues of selection bias, more recent impact studies have used various econometric techniques to deal with this problem. Methods commonly employed include instrumental variable approaches (e.g. Jones and Gibbon 2011; Parvathi and Waibel 2016; Chiputwa and Qaim 2016) and propensity score matching (Jena et al. 2012; Ruben and Fort 2012; Chiputwa, Spielman, and Qaim 2015; Ibanez and Blackman 2016; Ranjan Jena and Grote 2016). Available impact studies can also be grouped according to the selection of outcome variables, capturing economic, social, or ecological development outcomes.

\footnotetext{
${ }^{2}$ About 1.45 million smallholder farmers are members of Fairtrade producer organizations and 204 thousand workers are employed by Fairtrade hired labor organizations (Fairtrade International 2015).
} 
A large number of studies have examined economic implications of sustainability standards, looking at prices, revenues, incomes, or consumption expenditures. Many studies suggest that standards can create economic benefits for farmers (Bolwig, Gibbon, and Jones 2009; Jones and Gibbon 2011; Kleemann, Abdulai, and Buss 2014; Chiputwa, Spielman, and Qaim 2015; Parvathi and Waibel 2016; Jena, Stellmacher, and Grote 2017). Certified farmers often receive a price premium, contributing to higher revenues, incomes/expenditures, and reduced poverty rates (Bolwig, Gibbon, and Jones 2009; Chiputwa, Spielman, and Qaim 2015; Karki, Jena, and Grote 2016). However, various studies also suggest that economic effects are marginal or negative (Wollni and Zeller 2007; Valkila 2009; Jena et al. 2012; Ruben and Fort 2012). The price premium may not always be sufficiently high to cover investments required for standardcompliant production (Jena et al. 2012; Ibanez and Blackman 2016). Further, in some situations, farmers may not be able to sell their entire production within certified markets, given excess supply (Méndez, Bacon, Olson, Petchers et al. 2010; Ruben and Fort 2012). Mixed results suggest that contextual factors (region, crop, institutional environment, etc.) may influence economic outcomes. Further, effects may vary by standard, as standards differ in terms of their specific certification requirements (Ruben and Zuniga 2011; Chiputwa, Spielman, and Qaim 2015; van Rijsbergen et al. 2016; Jena, Stellmacher, and Grote 2017).

A few studies have looked at social outcomes such as health (Asfaw, Mithöfer, and Waibel 2010), social capital (Elder, Zerriffi, and Le Billon 2012), child education (Gitter et al. 2012), gender and household nutrition (Chiputwa and Qaim 2016), or multidimensional poverty measures (Ayuya et al. 2015). Most studies find positive effects. Yet, the evidence on social development goals is not conclusive, given the small number of studies and the specific selection of proxy variables used in each study.

Somewhat more studies have examined if sustainability standards promote environmental stewardship (e.g. Blackman and Naranjo 2012; Kleemann and Abdulai 2013; Elder, Zerriffi, and Le Billon 2013; Ibanez and Blackman 2016; Takahashi and Todo 2017), with overall promising results. For instance, Blackman and Naranjo (2012) found that certified farmers use more agro-ecological practices and less 'environmentally harmful' practices.

\subsection{Research gaps}

The number of impact studies on sustainability standards is growing. Yet, results are mixed and the evidence is not conclusive. We identified five research gaps, which are 
relevant from a development perspective: First, most available studies have focused on short term economic effects (e.g. prices, revenues, or income). Welfare outcomes such as household nutrition, child education, and gender equality have received very little scientific attention. This is a shortcoming because analyzing welfare effects beyond purely economic indicators is of particular importance for achieving the United Nation's Sustainable Development Goals (SDGs). Second, most available studies are based on cross-sectional data. ${ }^{3}$ As a result, issues of possible selection bias are more difficult to address. Further, measuring the effect of standards in a given year may not reflect longer-term effects, especially if only economic outcomes such as prices are considered. Third, most studies analyze the effect of one particular standard in one country. Consequently, comparisons between different standards in the same setting are hardly possible, limiting our understanding of dissimilar effects of different types of standards. Fourths, to our knowledge, all available, quantitative studies are based on householdlevel data. Issues of intra-household distribution of costs and benefits cannot be analyzed if the unit of observation is the household. From a development perspective, this is a shortcoming because standards - as other development interventions - may affect individuals within households in different ways (Doss 2001; Quisumbing et al. 2015). Likewise, heterogeneous treatment effects on female-headed and male-headed households have not been considered yet. Fifths, quantitative studies typically focus on the effect of sustainability standards on farmers. Farmers' preferences and attitudes have hardly received attention. Yet, it is known that farmers' preferences can influence their decision on which marketing channel to use, whether or not to participate in voluntary contractual agreements (Schipmann and Qaim 2011; Ochieng, Veettil, and Qaim 2017), or to adopt new farming practices (Ward et al. 2014; Marenya, Smith, and Nkonya 2014). By better addressing farmers' needs and preferences, adoption of sustainability standards may become more attractive and feasible for a larger number of farmers.

\subsection{Research objectives and approach}

This dissertation is composed of three papers, which address the aforementioned research gaps. All three papers are based on a farm household survey conducted in 2015 with smallholder coffee producers in Uganda. Most of the sample households were already interviewed in 2012 (by a different research team), allowing us to analyze some

\footnotetext{
${ }^{3}$ Exceptions include Parvathi and Waibel (2016) and van Rijsbergen et al. (2016).
} 
aspects based on panel data. ${ }^{4}$ Some of the sample households are Fairtrade, UTZ, or Organic certified; others are not certified under any standard. In each household, we interviewed male and female household members, to the extent possible. Our questionnaire covered farm, household, and contextual characteristics. We also collected detailed information on welfare indicators (e.g. asset ownership, household nutrition, and child education). Moreover, our survey included a choice experiment, which was conducted with male and female household members separately.

The first paper analyzes and compares the effect of Fairtrade and Organic ${ }^{5}$ standards, contributing to a better understanding of dissimilar welfare effects of sustainability standards. We focus on welfare outcomes that have received little attention so far, including food and non-food consumption expenditures, household nutrition, and child education. We hypothesize that Fairtrade and Organic have dissimilar effects on different areas of household welfare, because the two standards differ substantially in terms of their certification requirements. Our analysis is based on panel data and panel data econometric models, allowing us to reduce possible issues of selection bias.

The second paper is the first in-depth analysis of gendered implications of sustainability standards, based on quantitative data. We focus on Fairtrade and UTZ, ${ }^{6}$ because these standards include specific components on women's empowerment and gender equality. Given these gender components, we hypothesize that Fairtrade and UTZ may contribute to improving gender equality. We use cross-sectional data collected at the household and individual level. We analyze how Fairtrade and UTZ affect individuals in maleheaded households (i.e. male household heads and female spouses) and in femaleheaded households (i.e. female household heads). As outcomes, we consider different variables, capturing various areas of empowerment. These include gendered asset ownership, access to agricultural and financial services, and time allocation. For the statistical analysis, we use entropy balancing (Hainmueller 2012) to control for observed heterogeneity between certified and non-certified farmers. Entropy balancing

\footnotetext{
${ }^{4}$ We used the same questionnaire in 2012 and 2015. However, in 2015, we added some sections to the questionnaire. Two of the three papers are based on these new sections. Hence, we only use panel data for one (i.e. the first) paper.

${ }^{5}$ In this study, we disregard UTZ certified sample farmers. We focus on Fairtrade and Organic because these two standards are very dissimilar in terms of their specific requirements, as further explained in chapter 2. Given substantial difference in their design, we considered the comparison of Organic and Fairtrade particularly interesting.

${ }^{6}$ In this paper, we disregard Organic households. Organic standards do not include specific gender components, so that it unclear why the standard should affect gender relations. As we are interested in the effect of 'gender sensitive standards' we focus on UTZ and Fairtrade in this paper.
} 
is a novel weighting approach that can be employed to balance treatment and control groups in terms of their covariate distribution. To reduce possible bias from unobserved heterogeneity, we use estimates of farmers' willingness to accept (WTA) sustainability standards as a conditioning variable in reweighting control group observations. WTA estimates were obtained through a choice experiment, on which the third paper is based.

The third paper analyzes farmers' preferences for sustainability standards, based on choice experimental data. As mentioned, farmers' preferences have hardly received scientific attention. We are aware of only three choice experimental studies related to sustainability standards (Hope, Borgoyary, and Agarwal 2008; Ibnu et al. 2015; Vlaeminck et al. 2015). These studies look at concrete cases of existing standards, such as Fairtrade and Organic. We add to this literature by examining farmers' preferences for more generic, hypothetical attributes of sustainability standards, involving economic, social, and environmental components. Further, we employ a gendered research design: The choice experiment was conducted with male and female household members. Additionally, our choice experiment includes features of sustainability standards aimed at reducing gender inequality. Attributes included in the choice experiment are: (1) the price received for the coffee sold, (2) agricultural training, (3) gender policies, including credits for women and specific training on gender equality, (4) quality requirements and related harvesting and post-harvest management practices, (5) handling and use of chemical pesticides, and (6) record keeping. We hypothesize that farmers' preference for these attributes are heterogeneous, and may vary by gender, decision making power, and certification status.

\subsection{Outline}

The rest of this dissertation is organized as follows: Chapter 2 presents the first paper, analyzing the dissimilar effects of Fairtrade and Organic using panel data. In Chapter 3, the second paper is presented, which focusses on gendered implications of sustainability standards. The third paper, which explores farmers' preferences for sustainability standards based on choice experimental data, is presented in Chapter 4. Chapter 5 draws broader conclusions and policy implications, based on all three papers. Further, chapter 5 also discusses general limitations of the research design. The questionnaire used in 2015 is displayed in the general appendix. Details about the sampling procedure are included in the three papers themselves. 


\title{
2 Trading off nutrition and education? A panel data analysis of the dissimilar welfare effects of Organic and Fairtrade standards ${ }^{7}$
}

\begin{abstract}
Millions of smallholder farmers in developing countries participate in different types of sustainability standards. A growing body of literature has analyzed the welfare effects of such participation, with mixed results. Yet, there are important knowledge gaps. First, most existing studies look at the effects of one standard in one country. When comparing between studies, it is not clear whether dissimilar outcomes are driven by differences in standards or local conditions. Second, most studies use cross-section, observational data, so that selectivity issues remain a challenge. Third, the existing work has primarily analyzed effects in terms of purely economic indicators, such as income, ignoring other dimensions of household welfare. We address these shortcomings using panel data from small-scale coffee producers in Uganda and comparing the effects of two of the most popular sustainability standards, namely Organic and Fairtrade. Welfare effects are analyzed in terms of household expenditures, child education, and nutrition. Results show that Organic and Fairtrade both have positive effects on total consumption expenditures. However, notable differences are observed in terms of the other outcomes. Organic contributes to improved nutrition but has no effect on education. For Fairtrade it is exactly the other way around. We explore the mechanisms behind these differences.
\end{abstract}

Key words: certification, education, food standards, nutrition, panel data, welfare

\footnotetext{
7 This paper is co-authored by David Spielman (DS) and Matin Qaim (MQ). I (EM) developed the research idea, collected the survey data in 2015, did the data analysis, and wrote the paper. DS and MQ commented at the various stages of the research and contributed to writing and revising the paper.
} 


\subsection{Introduction}

Sustainability standards and certification schemes, such as Fairtrade and Organic, are gaining importance in international food markets. Often, these standards serve as a link between poor agricultural producers in developing countries and wealthy consumers in industrialized countries (Swinnen and Vandeplas 2011). Especially for higher-value foods, such as coffee, tea, or cocoa, rich-country consumers are increasingly concerned not only about product quality, but also about the environmental, social, and human rights implications during the process of production. Even though the details of sustainability standards are not always fully transparent, many consumers are willing to pay more for certified products with a sustainability label (Hoogland, Boer, and Boersema 2007; Grunert, Hieke, and Wills 2014; Potts et al. 2014; ITC 2015).

Depending on the particular standard, certification requirements may involve rules on environmentally friendly farming practices, democratic structures of farmer organizations, non-discrimination, or prohibition of child labor, just to name a few. Compliance is typically audited by an independent certification body. For farmers in developing counties, voluntary participation in such certification schemes can facilitate access to more lucrative export markets (Jones and Gibbon 2011; Kleemann, Abdulai, and Buss 2014; Chiputwa, Spielman, and Qaim 2015). However, meeting the requirements can be difficult and costly, especially for marginalized farmers (Handschuch, Wollni, and Villalobos 2013). In a smallholder context, group certification approaches are often encouraged, in order to reduce the cost for individuals (Bolwig, Gibbon, and Jones 2009; Chiputwa, Spielman, and Qaim 2015).

There is a growing literature about the impacts of sustainability standards on smallholder farmers in developing counties (e.g. Bolwig, Gibbon, and Jones 2009; Méndez, Bacon, Olson, Petchers et al. 2010; Jena et al. 2012; Chiputwa, Spielman, and Qaim 2015). Most existing studies focus on short-term economic indicators, such as prices or income, using cross-section data from a single year, often without properly establishing causality (Dragusanu, Giovannucci, and Nunn 2014). A few studies have looked at indicators beyond purely economic ones, including health, gender equality, child education, nutrition, and ecological sustainability (Arnould, Plastina, and Ball 2009; Gitter et al. 2012; Becchetti, Castriota, and Michetti 2013; Ibanez and Blackman 2016; Chiputwa and Qaim 2016). The results are fairly diverse, without conclusive evidence on whether or not sustainability standards actually promote rural development. Each study typically analyzes the effects of one particular standard in one country. Hence, comparisons between different standards in the same setting are hardly possible. 
Very few studies compare two or more standards, but these do not go beyond purely economic indicators (Méndez, Bacon, Olson, Petchers et al. 2010; Ruben and Zuniga 2011; Chiputwa, Spielman, and Qaim 2015; van Rijsbergen et al. 2016).

Our study adds to the existing literature in three ways. First, we analyze and compare the welfare effects of two popular sustainability standards, Fairtrade and Organic, in the same setting. The analysis builds on a survey of small-scale coffee producers in Uganda. Second, we use panel data collected in two survey rounds from the same farmers. Panel data models help to reduce selectivity issues and thus facilitate identification of causal effects. Third, we consider a set of outcome variables that capture several dimensions of household welfare, namely consumption expenditures, child education, and nutrition. A better understanding of the multidimensionality of impacts is important given that in the past the reduction in income poverty was more successful than the achievement of some of the other pressing development goals.

\subsection{Sustainability standards in theory and practice}

There are over 200 sustainability-oriented standards in use today (ITC 2016). In the coffee sector, 4C Association, Fairtrade, Organic, Rainforest Alliance, and UTZ are the most popular ones. Around 30 percent of the world's coffee production area was certified under one of these five standards in 2013 (ITC 2015). In this study, we focus on Fairtrade and Organic. The general principles of these two standards are briefly described in the following subsections, before an overview of expected and actual impacts on different dimensions of household welfare is provided. This overview further motivates the empirical analysis in subsequent sections.

\subsubsection{Fairtrade}

About 1.5 million smallholder farmers in developing countries are members of producer organizations that are certified by Fairtrade International. More than 50 percent of these farmers are coffee producers (Fairtrade International 2015).

Key features of the Fairtrade standard for small producer organizations are the Fairtrade minimum price and the Fairtrade premium. The Fairtrade minimum price is a floor price that becomes relevant whenever the world market price falls below a certain threshold. The Fairtrade premium is an additional amount of money paid to certified farmer organizations as an incentive for continued participation (Fairtrade International 2011b). In 2014, Fairtrade farmer organizations received an average premium of about 10,000 
US dollars, equivalent to about 70 dollars per member. Farmer organizations typically invest the premium in agricultural or marketing facilities, capacity development, and other economic services to their members. About 10 percent of the Fairtrade premium is used for social community projects, such as investments in health and education (Fairtrade International 2015).

Fairtrade farmer organizations are required to respect and promote principles such as non-discrimination, health and occupational safety, and the ban of child labor. Children under the age of 18 years must not be involved in exploitative or dangerous work. Further, children under the age of 15 cannot be employed by Fairtrade farmer organizations and cannot work on farms, except for times after school or during holidays. While Fairtrade primarily focuses on social and economic principles, the standard also promotes certain agricultural practices to protect the environment, such as integrated pest management and soil conservation measures (Fairtrade International 2011a).

\subsubsection{Organic}

Worldwide, about 2.3 million agricultural producers in 172 countries are certified Organic. The largest share of these producers ( 86 percent) lives in developing countries (FiBL and IFOAM 2016). There are various Organic standards; most are based on the rules of the International Federation of Organic Agriculture Movements (IFOAM).

Organic agriculture is based on the principles of health, ecology, fairness, and care (IFOAM 2014). While IFOAM also promotes certain social and economic objectives, certification requirements mainly focus on environmental issues. The application of chemical pesticides and synthetic fertilizers is banned. Further, farmers are trained to employ agricultural practices that improve and sustain soil fertility and nutrient cycles, such as intercropping, crop rotation, legume cultivation, and the use of organic fertilizers. Unlike Fairtrade, Organic certification is not associated with a guaranteed price premium. The expectation is rather that the market will reward farmers for complying with Organic principles.

\subsubsection{Possible economic impacts}

Certification is associated with a range of possible costs and benefits. Certification can be a tool to link farmers to higher-value export markets, which can be associated with higher and more stable prices (Kleemann, Abdulai, and Buss 2014; Chiputwa, Spielman, and Qaim 2015; Parvathi and Waibel 2016). However, not in all cases are 
average prices received by certified farmers higher than those received by their noncertified colleagues (Ruben and Fort 2012; Jena et al. 2012). Moreover, in some cases farmers cannot sell their entire harvest in certified value chains, due to excess supply of certified products (Méndez, Bacon, Olson, Petchers et al. 2010; Ruben and Fort 2012).

Beyond output price effects, certification may influence yields, product quality, or production costs in positive or negative ways through banning or encouraging the use of certain inputs, specific training of farmers, or the provision of credit, equipment, and marketing services (Becchetti and Costantino 2008; Bolwig, Gibbon, and Jones 2009; Méndez, Bacon, Olson, Petchers et al. 2010; Handschuch, Wollni, and Villalobos 2013). Required agricultural practices may sometimes also increase labor costs (Ibanez and Blackman 2016).

Costs and benefits of certification are often highly context-specific. Many studies focusing on Africa conclude that Organic and Fairtrade can have positive economic impacts (Bolwig, Gibbon, and Jones 2009; Jones and Gibbon 2011; Kleemann, Abdulai, and Buss 2014; Chiputwa, Spielman, and Qaim 2015). In Latin America, in contrast, studies sometimes find less positive effects of certification, especially in the coffee sector (Valkila 2009; Jena et al. 2012; Ruben and Fort 2012). Compared to Africa, the average input intensity in coffee production in Latin America, as well as mean yield and quality levels, are higher even without certification. Under those circumstances, certification may not further increase yield and quality levels.

\subsubsection{Possible impacts on child education}

Improvements in child education are an important mechanism to build up human capital, help households escape poverty in the medium and long run, and contribute to development more broadly. Private demand for education tends to increase with income. Hence, if farm households benefit economically from certification under a sustainability standard, they may decide to invest more in child education. Specifically, if Fairtrade or Organic standards increase incomes, households will find it easier to pay for school or tutor fees, learning materials, or school uniforms.

Beyond income gains, sustainability standards can also affect child education through other channels. As mentioned, Fairtrade restricts child labor, thus reducing the opportunity cost of time that children spend in school. Additionally, farmer organizations sometimes use parts of the Fairtrade premium to invest in educational programs. Bacon et al. (2008) describe how educational scholarships provided by a Fairtrade farmer organization in Nicaragua improved school attendance. Such programs 
can also raise awareness of the importance of child education in the community. Three studies have analyzed the impact of Fairtrade on child education in Latin America. Gitter et al. (2012) showed that Fairtrade certification increased schooling among secondary school children by 0.7 years. Arnould, Plastina, and Ball (2009) and Becchetti, Castriota, and Michetti (2013) found that children of Fairtrade producers are twice as likely to be enrolled in school as children of non-certified producers.

To our knowledge, there is no study that has looked at the effect of Organic certification on child education. Effects may possibly differ from those of Fairtrade. Organic standards do not explicitly address issues of child labor. Organic farming practices are often more labor-intensive. As a result, demand for child labor and thus the opportunity cost of time that children spend in school may possibly increase.

\subsubsection{Possible impacts on nutrition}

In smallholder farm households, agricultural products are partly sold and partly kept for home consumption. Certification can potentially affect household diets and nutrition through market and subsistence pathways. The market pathway will primarily be through higher cash revenues from agricultural sales. These additional cash revenues may then be used to purchase more - or more nutritious - foods. However, it is not guaranteed that households will actually use additional income from certified cash crop production to buy food. The literature suggests that income from different types of crops is sometimes earmarked for specific (non-food) purposes (Duflo and Udry 2004). Hence, the outcome will depend on the types of crops produced and sold, and also on who within the household controls the cash revenues.

The subsistence pathway may play a role because certification could affect the types of crops grown and livestock kept for home consumption. As mentioned, Organic farmers are encouraged to cultivate legumes, have longer crop rotations, and practice intercropping to enhance soil fertility and reduce pest infestation levels. Such measures tend to increase on-farm production diversity. Recent studies suggest a positive association between on-farm production diversity and dietary quality in smallholder farm households, especially in subsistence-oriented environments (Jones, Shrinivas, and Bezner-Kerr 2014; Sibhatu, Krishna, and Qaim 2015).

We are aware of only two studies that have explicitly analyzed the effects of sustainability certification on household diets and nutrition. Chiputwa and Qaim (2016) found that certification helps to improve dietary quality through positive effects on income and gender equality. Becchetti and Costantino (2008) also showed better-quality 
diets in certified households, which they attributed primarily to higher farm production diversity. Both studies looked at Organic and Fairtrade certified farms together, without disentangling the effects of each standard, as we do here. Given that Organic and Fairtrade have different principles, effects on diets and nutrition may differ as well.

\subsection{Study context}

\subsubsection{Coffee production in Uganda}

Coffee plays an important role in Uganda's economy. Coffee is one of the country's main foreign exchange earners and an important source of employment for the rural poor. About 3.5 million households depend on the coffee sector (UCDA 2016). Arabica and Robusta coffee are both grown in Uganda, but Robusta is more important, accounting for 85 percent of the country's coffee production. Robusta is grown at somewhat lower altitudes than Arabica, in regions up to 1200 meters above sea level (UCDA 2016). Given its lower quality, Robusta is traded at lower prices than Arabica. In general, world market prices for coffee can be quite volatile, even though prices paid to producers in Uganda were relatively stable in recent years (ICO 2016).

Robusta coffee is predominantly grown by smallholder farmers with land holdings between 0.5 and 2.5 hectares (UCDA 2016). Farmers typically rely on family labor. Access to agricultural inputs and extension services is limited. As a result, coffee yields are relatively low. In addition, poor-quality infrastructure, inappropriate storage practices, and lack of modern processing facilities limit the opportunities for value addition and the overall returns to coffee cultivation (ITC 2012).

Recently, the Ugandan government has promoted farmer participation in coffee certification schemes with the intention to increase the value of exports. The National Coffee Export Strategy has set a goal of increasing the amount of certified coffee by 5 percent each year (ITC 2012). Perhaps as a result, Uganda has the largest Organic certified area and the largest number of Organic producers among all countries in Africa. Organic coffee is grown on about 6 percent of Uganda's total area under this crop (FiBL and IFOAM 2016). Similarly, the number of Fairtrade certified farmers has also been growing in recent years. Currently, around 55,000 farmers and workers are Fairtrade certified in Uganda, most of them in the coffee sector (Fairtrade International 2015). 


\subsubsection{Panel survey}

The empirical analysis builds on two waves of a farm household survey that were conducted in 2012 and 2015. Households to be included were selected using a two-stage sampling strategy. In the first stage, two farmer organizations located in Luwero and Bukomansimbi (previously Masaka) districts, central Uganda, were purposively selected. Both organizations produce Robusta coffee and face similar agro-ecological conditions. One is certified under Fairtrade, the other under Organic. It is important to note, however, that not all members of these farmer organizations actually participate in certification. Whether or not to participate in certification remains a voluntary decision of individual households.

In the second sampling stage, in both farmer organizations certified and non-certified households were randomly selected based on complete member lists. In 2012, a total of 355 households were interviewed. In 2015, we conducted interviews with the same households, to the extent possible. Out of the original sample of 355 households, we were unable to interview 24 in 2015. To mitigate the effects of attrition, we replaced these 24 households with 24 other households that were also randomly selected (Hirano et al. 2001). Additionally, we increased the non-certified subsample by 30 additional, randomly selected households in 2015. For the analysis, we use the unbalanced panel including 409 households.

Table 1 provides an overview of the sample households by year and certification scheme. Certification is time-variant; farmers can enter or exit existing certification schemes as they wish. Out of the 331 households that were interviewed in both survey waves, 62 were Organic certified throughout, four newly entered, and 28 exited Organic certification between 2012 and 2015. Further, 103 households were Fairtrade certified in both years, 16 newly entered, and two exited the Fairtrade scheme between 2012 and 2015.

Table 1: Number of sampled households by year and certification status

\begin{tabular}{lcc}
\hline Certification status & 2012 & 2015 \\
\hline Not certified & 146 & 193 \\
Fairtrade certified & 108 & 121 \\
Organic certified & 101 & 71 \\
\hline Subtotal & 355 & 385 \\
Total & & 409 \\
\hline
\end{tabular}


Interviews were conducted by local enumerators, who were trained and supervised by the researchers. We used almost the same questionnaires in 2012 and 2015, covering a broad range of farm, household, and contextual characteristics. The interviews were conducted with the household heads. For diet and nutrition related questions (see details below), we also involved the main person in the household responsible for food purchases and cooking.

In addition to the household survey, in 2015 we also carried out focus group discussions with farmers and semi-structured interviews with key informants, such as agricultural extension officers, leaders of farmer organizations, coffee traders, and representatives of local development organizations. The purpose of these focus group discussions and interviews was to gain deeper insights into local conditions, coffee production, and farmers' perceptions of certification.

\subsubsection{Services of farmer organizations}

As explained, some of the coffee-producing households in our sample were certified under Fairtrade or Organic standards, while others were not. Irrespective of their certification status, households do not have binding contracts with the farmer organization or other buyers. That is, even certified farmers are free to sell their coffee in non-certified channels if they wish. This happens especially when price differences between certified and non-certified channels are small and cash is urgently needed. Most farmers sell their coffee as sundried cherries - either to middlemen or to the farmer organizations. The Fairtrade certified organization has an own facility to mill the coffee, thus being able to sell shelled green beans directly to exporters in Kampala.

Both farmer organizations offer additional services to their members, especially services related to agricultural training. The Fairtrade certified organization further operates its own input shop, where trained staff offers advice to farmers on how to apply fertilizers and pesticides. The Fairtrade certified organization also operates a credit scheme, which allows farmers to pre-finance inputs or make other types of farming investments. The input shop, the credit scheme, and also the milling facility were financed based on the Fairtrade premium received by the organization. 


\subsection{Empirical strategy}

Our objective is to analyze how Fairtrade and Organic certification affect different dimensions of household welfare, including consumption expenditures, child education, and nutrition. In this section, we explain the measurement of the outcome variables and the econometric modeling approaches used.

\subsubsection{Measurement of outcome variables}

We use consumption expenditures as a general proxy for household living standards (Klasen 2000). Consumption expenditures include the value of all food and non-food items consumed by household members. Data on non-food purchases were captured on an annual basis, referring to the 12 months prior to each survey wave. Food expenditures were calculated based on a seven-day food consumption recall at the household level. Food expenditures capture the value of all food items consumed, irrespective of whether the food was purchased, home-produced, or acquired from other sources. To aggregate food and non-food expenditures we converted both into daily values expressed in Ugandan shillings (UGX). The official consumer price index was used to adjust for inflation between the two survey waves (World Bank 2016).

To measure child education, the survey questionnaire included a special section on education related expenditures (school or tutor fees, uniforms, learning materials, transportation costs to reach the school etc.). Public schools are free in Uganda, but uniforms and learning materials (pencils, notebooks etc.) have to be purchased. Otherwise, children are not allowed to attend classes. Further, tutorials (extra classes) are common in Uganda and have to be paid for. Some parents also decide to send their children to private schools, which are generally considered better but charge tuition fees. We therefore expect expenditures to be a good proxy of the quantity and quality of actual education received. To be comparable, we divided household expenditures on education by the number of children in primary and secondary school age.

Education expenditures at the household level were collected in 2012 and 2015. To further increase precision, in 2015 we additionally collected individual-level education expenditures for each child living in the household. These individual-level expenditure data, as well as the number of schooling years completed by each child, are used as additional proxies of child education. 
Outcomes in terms of diets and nutrition are measured based on the seven-day consumption recall, covering quantities and values of more than 100 different food items. A first indicator we use is food expenditures, calculated as explained above. In addition, we converted the quantities of food items consumed into energy and nutrient levels, which is a common approach to analyze issues of household food security and dietary quality (de Haen, Klasen, and Qaim 2011; Chege, Andersson, and Qaim 2015). We used local food composition tables for Uganda for these calculations (Hotz, Lubowa, and Sison 2012). In terms of nutrients, we focus on iron, zinc, and vitamin A, because deficiencies in these micronutrients cause large public health problems in many developing countries (Black et al. 2008). To account for the fact that requirements differ by age and gender, quantities consumed at the household level are expressed per adult equivalent (AE). We classify households as deficient when the calculated daily consumption level remains below international recommendations (FAO, WHO, and UNU). ${ }^{8}$

\subsubsection{Panel regression models}

To analyze the effects of Fairtrade and Organic certification on household welfare, we estimate panel regression models of the following type:

$$
Y_{i t}=\beta_{0}+\beta_{1} \text { Certified }_{i t}+\beta_{2} X_{i t}+\beta_{3} Z_{i}+\varepsilon_{i t}
$$

where $Y_{i t}$ represents the different welfare measures referring to household $i$ in year $t$. We estimate different models for each welfare indicator. Certified is the treatment dummy that takes a value of one when the household is certified under Fairtrade or Organic, and zero otherwise. As mentioned, the certification status can vary over time. $X_{i t}$ and $Z_{i}$ are vectors of time-variant and time-invariant farm, household, and contextual characteristics.

In additional models, we further disaggregate the treatment variable as follows:

$$
Y_{i t}=\beta_{0}+\beta_{1} \text { Fairtrade }_{i t}+\beta_{2} \text { Organic }_{i t}+\beta_{3} X_{i t}+\beta_{4} Z_{i}+\varepsilon_{i t}
$$

\footnotetext{
${ }^{8}$ Recommended consumption levels per AE and day are as follows: $2400 \mathrm{kcal}$ for energy; $18 \mathrm{mg}$ for iron; $15 \mathrm{mg}$ for zinc; $625 \mu \mathrm{g}$ retinol equivalents for vitamin A.
} 
where Organic and Fairtrade are two treatment dummies, which are mutually exclusive in our case because none of the farmers in our sample is certified under both standards. The models in equation (2) allow us to identify possible differences in impacts between Fairtrade and Organic.

The models in equations (1) and (2) are estimated with random effects (RE) and fixed effects (FE) estimators. RE estimators are more efficient, but can lead to biased estimates of the treatment effect when unobserved factors are jointly correlated with certification and the welfare outcomes. This is tested with a Hausman test. FE estimators control for time-invariant unobserved heterogeneity, so that the treatment effect estimates suffer less from possible selection bias.

For the models with continuous dependent variables (consumption expenditures, food expenditures, non-food expenditures, education expenditures), we use log-linear specifications. For the models with dummy dependent variables (energy and micronutrient deficiencies), we use probit specifications. As probit models cannot be estimated with FE estimators, we additionally use linear probability models as robustness checks.

\subsubsection{Cross-section models}

For the individual level child education variables we only have cross-section data, as these were only collected in 2015 . For these outcome variables, we estimate regression models of the following type:

$$
Y_{j i}=\beta_{0}+\beta_{1} \text { Fairtrade }_{i}+\beta_{2} \text { Organic }_{i}+\beta_{3} C_{j i}+\beta_{3} H_{i}+\varepsilon_{j i}
$$

where $Y_{j i}$ represents the education indicator referring to child $j$ in household $i . C_{j i}$ and $H_{i}$ are child level and household level control variables. Organic and Fairtrade are the two treatment dummies, as before. All variables in equation (3) refer to 2015.

Education expenditure per child is a continuous dependent variable, for which we use a log-linear specification. Individual years of schooling is a count variable, for which we use a Poisson model. ${ }^{9}$ Since many households have more than one child, we estimate standard errors with cluster correction at the household level.

\footnotetext{
${ }^{9}$ We tested if the data follow a Poisson distribution and detected no overdispersion.
} 
One problem with the cross-section models in equation (3) is that the estimated treatment effects for Fairtrade and Organic may suffer from selection bias due to unobserved heterogeneity. To test and control for such bias, we use an instrumental variable (IV) approach. As both treatment variables may potentially be endogenous, we need at least two instruments that are correlated with certification but uncorrelated with child education.

We use distance from the farm household to the main building of the Fairtrade organization as an instrument for Fairtrade certification. As is shown in table A1 in the appendix, households located closer to this building are more likely to be Fairtrade certified. This is plausible because the building is where the staff of the farmer organization (management, extension officers etc.) and also the coffee milling facility are based. Closeness means that households are more exposed and have better access to Fairtrade activities and services. On the other hand, distance to the Fairtrade building does not influence child education through pathways other than certification. This was tested by regressing the education variables on the instrument and other controls for the subsample of non-certified households (table A2 in the appendix). One might have expected that the building of the Fairtrade organization is located in a setting with good infrastructure, which could improve households' access to education through various channels. But this is not the case. The building is located in the coffee growing area, away from tarmac roads and not close to schools or other public services.

We use altitude of the farm location as an instrument for Organic certification. Altitude has been used previously as an instrument for certification (Chiputwa and Qaim 2016). While differences in altitudes in our sample are relatively small, altitude is negatively correlated with Organic certification (table A1, appendix). This is probably related to clustering effects. On the other hand, altitude does not directly influence education (table A2, appendix). Unlike Arabica coffee, the quality of Robusta is less influenced by altitude. Altitude differences in our sample have no direct effect on coffee prices, household income levels, or other variables that would affect child education.

\subsection{Results and discussion}

\subsubsection{Descriptive statistics}

Table 2 provides descriptive statistics. Organic, Fairtrade, and non-certified households differ significantly in terms of various characteristics. Heads of non-certified households are significantly younger than heads of Organic and Fairtrade certified 
households. Organic households are more often headed by females than non-certified households. Fairtrade household heads are better educated than their non-certified counterparts.

Table 2: Descriptive statistics by certification status (pooled data for 2012 and 2015)

\begin{tabular}{|c|c|c|c|c|}
\hline & $\begin{array}{c}\text { (1) } \\
\text { Full sample }\end{array}$ & $\begin{array}{c}(2) \\
\text { Not certified }^{\mathrm{a}}\end{array}$ & $\begin{array}{c}(3) \\
\text { Fairtrade }^{b}\end{array}$ & $\begin{array}{c}(4) \\
\text { Organic }^{c}\end{array}$ \\
\hline \multicolumn{5}{|l|}{ Household, farm, contextual characteristics } \\
\hline Household size (AE) & $\begin{array}{c}4.92 \\
(2.54)\end{array}$ & $\begin{array}{l}4.64^{* * *} \\
(2.51)\end{array}$ & $\begin{array}{c}4.90 \\
(2.21)\end{array}$ & $\begin{array}{l}5.50^{* * *} \\
(2.91)\end{array}$ \\
\hline Female headed household (1/0) & $\begin{array}{c}0.22 \\
(0.42)\end{array}$ & $\begin{array}{c}0.21 \\
(0.41)\end{array}$ & $\begin{array}{c}0.18 \\
(0.39)\end{array}$ & $\begin{array}{l}0.31^{\text {*** }} \\
(0.47)\end{array}$ \\
\hline Household head schooling (yrs.) & $\begin{array}{c}6.63 \\
(3.64)\end{array}$ & $\begin{array}{c}6.48 \\
(3.46)\end{array}$ & $\begin{array}{l}7.92^{* * *} \\
(3.47)\end{array}$ & $\begin{array}{l}5.22^{* * *} \\
(3.65)\end{array}$ \\
\hline Household head age (yrs.) & $\begin{array}{c}53.14 \\
(14.20)\end{array}$ & $\begin{array}{c}49.40^{\text {*** }} \\
(14.99)\end{array}$ & $\begin{array}{l}56.60^{* * * *} \\
(12.27)\end{array}$ & $\begin{array}{l}55.89^{* * *} \\
(13.23)\end{array}$ \\
\hline Distance to tarmac road $(\mathrm{km})$ & $\begin{array}{c}17.74 \\
(10.23)\end{array}$ & $\begin{array}{l}18.58^{* *} \\
(12.96)\end{array}$ & $\begin{array}{c}14.19^{\text {**** }} \\
(6.19)\end{array}$ & $\begin{array}{c}20.82^{* *} \\
(6.45)\end{array}$ \\
\hline \multicolumn{5}{|l|}{ Household expenditures (UGX) } \\
\hline Food and non-food expenditures /AE/day & $\begin{array}{c}4938.65 \\
(2679.73)\end{array}$ & $\begin{array}{c}4535.90^{* * * *} \\
(2651.40)\end{array}$ & $\begin{array}{c}5611.70^{* * * *} \\
(2808.67)\end{array}$ & $\begin{array}{c}4836.32 \\
(2383.49)\end{array}$ \\
\hline Non-food expenditure /AE/day & $\begin{array}{c}1659.65 \\
(1659.25)\end{array}$ & $\begin{array}{l}1420.29^{* * *} \\
(1554.91)\end{array}$ & $\begin{array}{c}2368.51^{* * *} \\
(1966.63)\end{array}$ & $\begin{array}{l}1187.63^{*} \\
(975.51)\end{array}$ \\
\hline Food expenditure /AE/day & $\begin{array}{c}3279.00 \\
(1794.33)\end{array}$ & $\begin{array}{l}3115.61^{* *} \\
(1758.57)\end{array}$ & $\begin{array}{c}3243.19 \\
(1725.29)\end{array}$ & $\begin{array}{c}3648.70^{* * * *} \\
(1907.95)\end{array}$ \\
\hline Total expenditure on education & $\begin{array}{c}3202.34 \\
(5120.05)\end{array}$ & $\begin{array}{l}2079.83^{* * *} \\
(3848.00)\end{array}$ & $\begin{array}{l}5276.91^{* * *} \\
(6931.11)\end{array}$ & $\begin{array}{c}2652.63^{*} \\
(3377.05)\end{array}$ \\
\hline \multicolumn{5}{|l|}{ Household nutrition } \\
\hline Energy deficiency $(1 / 0)^{d}$ & $\begin{array}{c}0.37 \\
(0.48)\end{array}$ & $\begin{array}{c}0.38 \\
(0.49)\end{array}$ & $\begin{array}{c}0.44 \\
(0.50)\end{array}$ & $\begin{array}{l}0.25^{* * *} \\
(0.43)\end{array}$ \\
\hline Iron deficiency $(1 / 0)^{\mathrm{e}}$ & $\begin{array}{c}0.48 \\
(0.50)\end{array}$ & $\begin{array}{c}0.51 \\
(0.50)\end{array}$ & $\begin{array}{c}0.57 \\
(0.50)\end{array}$ & $\begin{array}{l}0.29^{* * * *} \\
(0.45)\end{array}$ \\
\hline Zinc deficiency $(1 / 0)^{f}$ & $\begin{array}{c}0.78 \\
(0.42)\end{array}$ & $\begin{array}{c}0.79 \\
(0.41)\end{array}$ & $\begin{array}{c}0.84 \\
(0.37)\end{array}$ & $\begin{array}{l}0.66^{* * * *} \\
(0.47)\end{array}$ \\
\hline Vitamin A deficiency $(1 / 0)^{\mathrm{g}}$ & $\begin{array}{c}0.39 \\
(0.49) \\
\end{array}$ & $\begin{array}{l}0.42^{*} \\
(0.50)\end{array}$ & $\begin{array}{c}0.49 \\
(0.50)\end{array}$ & $\begin{array}{l}0.19^{* * *} \\
(0.39)\end{array}$ \\
\hline Observations & 740 & 339 & 229 & 172 \\
\hline $\begin{array}{l}\text { a Significance level in this column refers } \\
\text { schemes) and the control group. } \\
\text { b Significance level in this column refers } \\
\text { group. } \\
\text { b Significance level in this column refers } \\
\text { group. } \\
\text { d } \text { Energy consumption }<2400 \mathrm{kcal} / \mathrm{AE} / \mathrm{day} \\
{ }^{\mathrm{e}} \text { Iron consumption }<18.27 \mathrm{mg} / \mathrm{AE} / \mathrm{day} \\
{ }^{\mathrm{f}} \text { Zinc consumption }<15 \mathrm{mg} / \mathrm{AE} / \mathrm{day} \\
{ }^{\mathrm{g}} \text { Vitamin A consumption }<625 \mu \mathrm{g} \text { RE/AF } \\
\text { Standard deviations in parentheses. }{ }^{*} p<0\end{array}$ & $\begin{array}{l}\text { e difference } \\
\text { e difference } \\
\text { he difference }\end{array}$ & $\begin{array}{l}\text { etween scheme } \\
\text { etween Fairtrad } \\
\text { etween Organic }\end{array}$ & $\begin{array}{l}\text { participants } \\
\text { participants } \\
\text { participants }\end{array}$ & $\begin{array}{l}\text { all certification } \\
\text { and the control } \\
\text { and the control }\end{array}$ \\
\hline
\end{tabular}


Table 2 also reveals that the three groups differ significantly in terms of the welfare indicators. While Fairtrade households have higher non-food expenditures, Organic households spend more on food per AE. And higher food expenditures in Organic households are associated with lower levels of energy and micronutrient deficiencies.

\subsubsection{Regression results}

We start the regression analysis by providing an overview of the impact of certification in general, before focusing on the differences between Fairtrade and Organic. The first set of regression estimates is shown in table 3. For each model, results with RE and FE estimators are shown. The test statistics, which are displayed in the lower part of the table, reject the null hypothesis of zero correlation with the error term, so we focus on the FE results for interpretation.

The first two columns of table 3, which are estimates of the model explained in equation (1), suggest that certification increases household welfare in terms of total consumption expenditures. Columns (3) and (4) show estimates of the model in equation (2), with disaggregation of the treatment variable by certification scheme. The effects of Fairtrade and Organic are very similar. The coefficient of 0.32 for Fairtrade in column (4) implies that Fairtrade certification increases total consumption expenditure by 37 percent. ${ }^{10}$ Organic certification increases expenditure by about 36 percent.

Looking at food and non-food expenditures separately (columns 5-12), we find that Fairtrade certification more than doubles non-food expenditures, but has no significant effect on food expenditures. The opposite holds for Organic certification, which increases food expenditures by almost 30 percent, but has no significant effect on nonfood expenditures. Below, we discuss possible reasons for the dissimilar impact of Fairtrade and Organic.

\footnotetext{
${ }^{10}$ The percentage effect of dummy variables in log-linear models is calculated as $[\exp (\hat{\beta})-1] \times 100$.
} 
Table 3: Effects of certification on household expenditure (panel data models)

\begin{tabular}{|c|c|c|c|c|c|c|c|c|c|c|c|c|}
\hline & \multicolumn{4}{|c|}{ Total expenditure } & \multicolumn{4}{|c|}{ Non-food expenditure } & \multicolumn{4}{|c|}{ Food expenditure } \\
\hline & (1) & (2) & (3) & (4) & (5) & (6) & (7) & (8) & (9) & (10) & (11) & (12) \\
\hline & RE & FE & RE & FE & $\mathrm{RE}$ & $\mathrm{FE}$ & $\mathrm{RE}$ & $\mathrm{FE}$ & RE & $\mathrm{FE}$ & $\mathrm{RE}$ & $\mathrm{FE}$ \\
\hline Certified (1/0) & $\begin{array}{l}0.21^{* * * *} \\
(0.04)\end{array}$ & $\begin{array}{l}0.31^{* * * *} \\
(0.08)\end{array}$ & & & $\begin{array}{l}0.36^{* * * *} \\
(0.07)\end{array}$ & $\begin{array}{l}0.49^{* * * *} \\
(0.17)\end{array}$ & & & $\begin{array}{l}0.14^{* * * *} \\
(0.04)\end{array}$ & $\begin{array}{l}0.22^{* * *} \\
(0.08)\end{array}$ & & \\
\hline Fairtrade $(1 / 0)$ & & & $\begin{array}{l}0.24^{* * * *} \\
(0.05)\end{array}$ & $\begin{array}{l}0.32^{* *} \\
(0.14)\end{array}$ & & & $\begin{array}{c}0.62^{* * * *} \\
(0.09)\end{array}$ & $\begin{array}{l}0.82^{* * *} \\
(0.41)\end{array}$ & & & $\begin{array}{c}0.06 \\
(0.04)\end{array}$ & $\begin{array}{c}0.14 \\
(0.14)\end{array}$ \\
\hline Organic (1/0) & & & $\begin{array}{l}0.18^{* * * *} \\
(0.05)\end{array}$ & $\begin{array}{c}0.31^{\text {*** }} \\
(0.11)\end{array}$ & & & $\begin{array}{c}0.06 \\
(0.08)\end{array}$ & $\begin{array}{c}0.29 \\
(0.20)\end{array}$ & & & $\begin{array}{l}0.22^{* * * *} \\
(0.05)\end{array}$ & $\begin{array}{l}0.26^{* *} \\
(0.11)\end{array}$ \\
\hline Year=2015 & $\begin{array}{l}0.09^{* * * *} \\
(0.03)\end{array}$ & $\begin{array}{c}0.10^{* * * *} \\
(0.04)\end{array}$ & $\begin{array}{c}0.09^{* * * *} \\
(0.03)\end{array}$ & $\begin{array}{c}0.10^{* * * *} \\
(0.04)\end{array}$ & $\begin{array}{l}0.39^{* * * *} \\
(0.05)\end{array}$ & $\begin{array}{l}0.39^{* * * *} \\
(0.06)\end{array}$ & $\begin{array}{c}0.37^{* * * *} \\
(0.05)\end{array}$ & $\begin{array}{c}0.36^{* * * *} \\
(0.06)\end{array}$ & $\begin{array}{l}-0.05 \\
(0.03)\end{array}$ & $\begin{array}{l}-0.05 \\
(0.04)\end{array}$ & $\begin{array}{l}-0.05 \\
(0.03)\end{array}$ & $\begin{array}{l}-0.04 \\
(0.04)\end{array}$ \\
\hline Household size (AE) & $\begin{array}{c}-0.14^{* * * *} \\
(0.02)\end{array}$ & $\begin{array}{c}-0.16^{* * * *} \\
(0.03)\end{array}$ & $\begin{array}{c}-0.14^{* * * *} \\
(0.02)\end{array}$ & $\begin{array}{c}-0.16^{* * * *} \\
(0.03)\end{array}$ & $\begin{array}{c}-0.11^{* * * *} \\
(0.04)\end{array}$ & $\begin{array}{c}-0.18^{* * *} \\
(0.07)\end{array}$ & $\begin{array}{c}-0.11^{* * * *} \\
(0.04)\end{array}$ & $\begin{array}{c}-0.19^{* * * *} \\
(0.07)\end{array}$ & $\begin{array}{c}-0.15^{* * * *} \\
(0.02)\end{array}$ & $\begin{array}{c}-0.16^{* * * *} \\
(0.04)\end{array}$ & $\begin{array}{c}-0.15^{* * *} \\
(0.02)\end{array}$ & $\begin{array}{c}-0.15^{* * * *} \\
(0.04)\end{array}$ \\
\hline Household size squared & $\begin{array}{l}0.01^{* * * *} \\
(0.00)\end{array}$ & $\begin{array}{l}0.00^{* * *} \\
(0.00)\end{array}$ & $\begin{array}{l}0.01^{\text {**** }} \\
(0.00)\end{array}$ & $\begin{array}{l}0.00^{* * *} \\
(0.00)\end{array}$ & $\begin{array}{c}0.00 \\
(0.00)\end{array}$ & $\begin{array}{c}0.00 \\
(0.00)\end{array}$ & $\begin{array}{c}0.00 \\
(0.00)\end{array}$ & $\begin{array}{c}0.00 \\
(0.00)\end{array}$ & $\begin{array}{l}0.01^{* * * *} \\
(0.00)\end{array}$ & $\begin{array}{l}0.00^{*} \\
(0.00)\end{array}$ & $\begin{array}{l}0.01^{* * *} \\
(0.00)\end{array}$ & $\begin{array}{l}0.00^{*} \\
(0.00)\end{array}$ \\
\hline Female headed $(1 / 0)$ & $\begin{array}{l}-0.11^{\text {** }} \\
(0.05)\end{array}$ & $\begin{array}{c}-0.52^{* * * *} \\
(0.18)\end{array}$ & $\begin{array}{l}-0.11^{\text {*** }} \\
(0.05)\end{array}$ & $\begin{array}{c}-0.52^{* * * *} \\
(0.18)\end{array}$ & $\begin{array}{c}-0.29^{* * *} \\
(0.10)\end{array}$ & $\begin{array}{l}-0.96^{* *} \\
(0.48)\end{array}$ & $\begin{array}{c}-0.25^{* * * *} \\
(0.09)\end{array}$ & $\begin{array}{c}-0.95^{* *} \\
(0.46)\end{array}$ & $\begin{array}{l}-0.06 \\
(0.05)\end{array}$ & $\begin{array}{c}-0.41^{* * * *} \\
(0.15)\end{array}$ & $\begin{array}{l}-0.07 \\
(0.05)\end{array}$ & $\begin{array}{c}-0.41^{* * *} \\
(0.16)\end{array}$ \\
\hline Household head school (yrs.) & $\begin{array}{l}0.02^{* * * *} \\
(0.01)\end{array}$ & $\begin{array}{l}-0.01 \\
(0.01)\end{array}$ & $\begin{array}{c}0.02^{* * * *} \\
(0.01)\end{array}$ & $\begin{array}{l}-0.01 \\
(0.01)\end{array}$ & $\begin{array}{l}0.05^{* * * *} \\
(0.01)\end{array}$ & $\begin{array}{l}-0.02 \\
(0.02)\end{array}$ & $\begin{array}{l}0.04^{* * * *} \\
(0.01)\end{array}$ & $\begin{array}{l}-0.02 \\
(0.02)\end{array}$ & $\begin{array}{c}0.01 \\
(0.01)\end{array}$ & $\begin{array}{c}0.01 \\
(0.02)\end{array}$ & $\begin{array}{l}0.01^{* * *} \\
(0.01)\end{array}$ & $\begin{array}{c}0.01 \\
(0.02)\end{array}$ \\
\hline Household head age (yrs.) & $\begin{array}{l}0.00^{* * *} \\
(0.00)\end{array}$ & $\begin{array}{l}-0.01^{*} \\
(0.01)\end{array}$ & $\begin{array}{l}0.00^{* * *} \\
(0.00)\end{array}$ & $\begin{array}{l}-0.01^{*} \\
(0.01)\end{array}$ & $\begin{array}{l}-0.00 \\
(0.00)\end{array}$ & $\begin{array}{l}-0.02 \\
(0.01)\end{array}$ & $\begin{array}{l}-0.00 \\
(0.00)\end{array}$ & $\begin{array}{l}-0.02 \\
(0.01)\end{array}$ & $\begin{array}{c}0.00^{* * * *} \\
(0.00)\end{array}$ & $\begin{array}{l}-0.01^{*} \\
(0.00)\end{array}$ & $\begin{array}{l}0.00^{* * * *} \\
(0.00)\end{array}$ & $\begin{array}{l}-0.01^{*} \\
(0.00)\end{array}$ \\
\hline Distance to road $(\mathrm{km})$ & $\begin{array}{c}0.00 \\
(0.00)\end{array}$ & $\begin{array}{l}-0.00 \\
(0.00)\end{array}$ & $\begin{array}{c}0.00 \\
(0.00)\end{array}$ & $\begin{array}{l}-0.00 \\
(0.00)\end{array}$ & $\begin{array}{c}0.00 \\
(0.00)\end{array}$ & $\begin{array}{l}-0.00 \\
(0.01)\end{array}$ & $\begin{array}{l}0.01^{* * *} \\
(0.00)\end{array}$ & $\begin{array}{l}-0.00 \\
(0.01)\end{array}$ & $\begin{array}{l}0.00^{* *} \\
(0.00)\end{array}$ & $\begin{array}{c}0.00 \\
(0.00)\end{array}$ & $\begin{array}{c}0.00 \\
(0.00)\end{array}$ & $\begin{array}{c}0.00 \\
(0.00)\end{array}$ \\
\hline Constant & $\begin{array}{l}8.43^{* * * *} \\
(0.10) \\
\end{array}$ & $\begin{array}{l}9.52^{* * *} \\
(0.33)\end{array}$ & $\begin{array}{l}8.44^{* * * *} \\
(0.11)\end{array}$ & $\begin{array}{l}9.52^{* * *} \\
(0.33)\end{array}$ & $\begin{array}{l}6.77^{* * * *} \\
(0.20)\end{array}$ & $\begin{array}{l}8.56^{* * * *} \\
(0.64)\end{array}$ & $\begin{array}{l}6.83^{* * *} \\
(0.20) \\
\end{array}$ & $\begin{array}{l}8.53^{* * * *} \\
(0.64)\end{array}$ & $\begin{array}{l}8.18^{* * * *} \\
(0.10)\end{array}$ & $\begin{array}{l}8.94^{* * * *} \\
(0.30)\end{array}$ & $\begin{array}{l}8.15^{* * *} \\
(0.10)\end{array}$ & $\begin{array}{l}8.95^{* * *} \\
(0.30)\end{array}$ \\
\hline No. of observations & 740 & 740 & 740 & 740 & 740 & 740 & 740 & 740 & 740 & 740 & 740 & 740 \\
\hline No. of households & 409 & 409 & 409 & 409 & 409 & 409 & 409 & 409 & 409 & 409 & 409 & 409 \\
\hline F-value & & $18.72^{* * *}$ & & $16.85^{\text {*** }}$ & & $17.97^{* * *}$ & & $15.48^{* * *}$ & & $18.72^{* * *}$ & & $7.93^{\text {*** }}$ \\
\hline Wald $\chi^{2}$ & $231.04^{* * * *}$ & & $229.74^{* * * *}$ & & $170.77^{* * * *}$ & & $197.28^{* * * *}$ & & $168.87^{* * *}$ & & $178.69^{* * *}$ & \\
\hline Hausman test $\chi^{2}$ & $29.68^{* * *}$ & & $30.46^{* * *}$ & & $30.56^{* * *}$ & & $33.77^{* * *}$ & & $14.84^{*}$ & & 13.93 & \\
\hline Sargan-Hansen test $\chi^{2}$ & $37.47^{* * * *}$ & & $38.15^{* * *}$ & & $40.27^{* * *}$ & & $44.17^{* * *}$ & & $17.85^{* *}$ & & $16.95^{* *}$ & \\
\hline
\end{tabular}

Cluster robust standard errors in parentheses. The dependent variable in all models is the logarithm of expenditure per adult equivalent (AE). RE, random effects. FE, fixed effects.

$p<0.1,{ }^{* *} p<0.05,{ }^{* * *} p<0.01$ 


\subsubsection{Fairtrade certified households}

Certification may help to increase coffee yields, quality, and income through improving farmers' access to technology, inputs, knowledge, and higher-value output markets. However, the particular requirements and services offered differ by certification scheme, so that the concrete effects may differ too. As explained, the Fairtrade farmer organization provides a number of services to its members, such as agricultural training, provision of credits, value addition through its own coffee mill, and easier access to inputs through the organizations' farm input shop.

Indeed, Fairtrade certified farmers in our sample use more productivity-enhancing inputs than Organic certified or non-certified farmers (table 4). Better production and marketing conditions for coffee also seem to contribute to a higher degree of specialization. Figure 1 (panel A) shows that Fairtrade farmers use a larger share of their total land for coffee production than the other two subsamples. These observations are in line with research on Fairtrade effects in other countries and regions (Ruben and Zuniga 2011; Ruben and Fort 2012).

Table 4: Farming practices by certification status (pooled data for 2012 and 2015)

\begin{tabular}{|c|c|c|c|}
\hline & $\begin{array}{c}(1) \\
\text { Not certified }^{\mathrm{a}}\end{array}$ & $\begin{array}{c}(2) \\
\text { Fairtrade }^{b} \\
\end{array}$ & $\begin{array}{c}(3) \\
\text { Organic }^{c}\end{array}$ \\
\hline \multirow{3}{*}{ Use of pesticides } & \multicolumn{3}{|c|}{ Share of households } \\
\hline & $0.44^{* *}$ & $0.58^{* * * *}$ & $0.08^{* * *}$ \\
\hline & $(0.50)$ & $(0.49)$ & $(0.27)$ \\
\hline \multirow[t]{2}{*}{ Use of chemical fertilizers } & 0.17 & $0.34^{* * *}$ & $0.01^{* * * *}$ \\
\hline & $(0.37)$ & $(0.48)$ & $(0.08)$ \\
\hline \multirow[t]{2}{*}{ Cultivation of legumes } & 0.87 & 0.88 & $0.93^{* *}$ \\
\hline & $(0.34)$ & $(0.33)$ & $(0.26)$ \\
\hline Observations & 339 & 229 & 172 \\
\hline \multicolumn{4}{|c|}{$\begin{array}{l}\text { Significance level in this column refers to the difference between non-certified households and all } \\
\text { certified households combined. } \\
\text { b Significance level in this column refers to the difference between Fairtrade and non-certified } \\
\text { households. } \\
{ }^{c} \text { Significance level in this column refers to the difference between Organic and non-certified households. }\end{array}$} \\
\hline
\end{tabular}

Figure 1 (panel B) shows that Fairtrade farmers also receive higher average coffee prices than their Organic certified and non-certified counterparts. To some extent, this may be due to the guaranteed Fairtrade minimum price. However, even when Fairtrade farmers sell in non-certified channels, they often fetch higher prices due to better quality and higher levels of processing. The key informant interviews with traders and other stakeholders of the coffee value chain confirmed that the coffee from Fairtrade farmers 
is generally considered of high quality in the local context. Similar observations were also made elsewhere (Ruben and Fort 2012; van Rijsbergen et al. 2016).

Figure 1: Differences by certification status

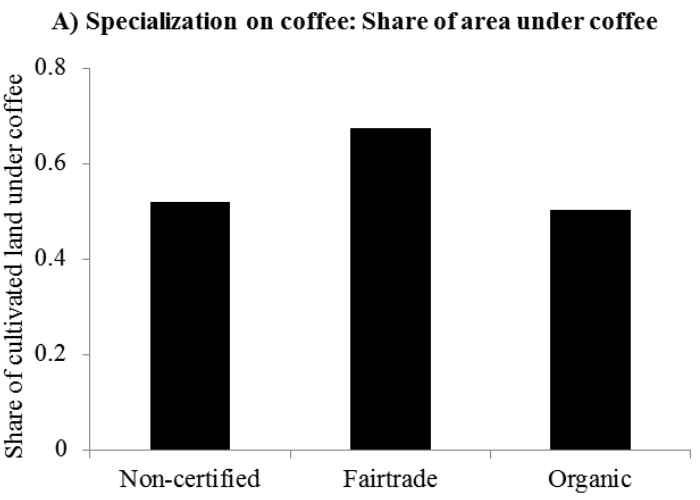

B) Median coffee prices

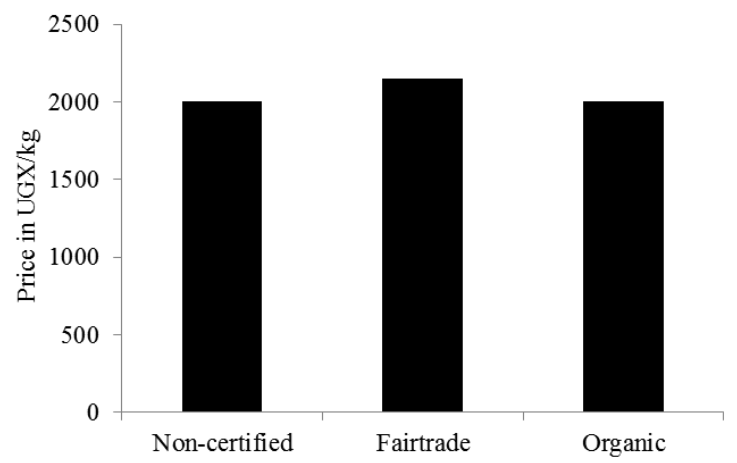

C) Production diversity: No. of crop and livestock species

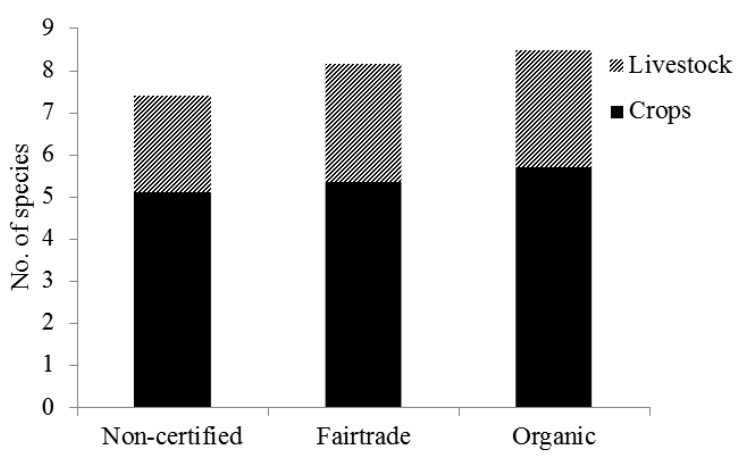

D) Market orientation: No. of crops sold (partly or completely)

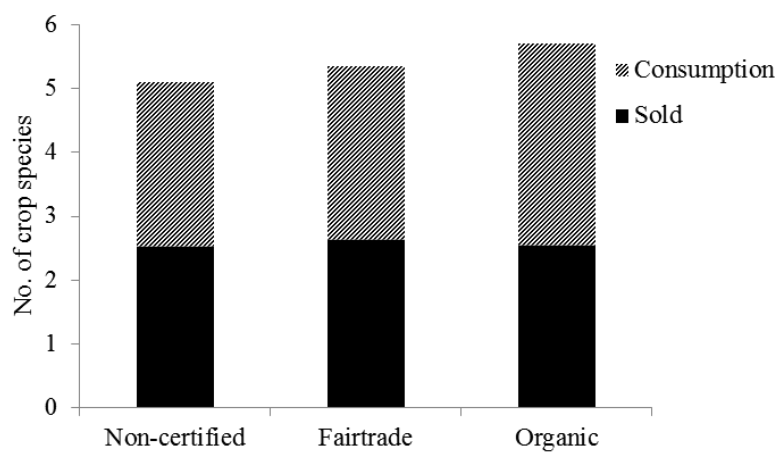

The effects discussed so far can explain why Fairtrade farmers have higher incomes from coffee production than the other two groups, but why is this income spent more on non-food goods and services than on food? This question can be answered by analyzing the utilization of different types of income (Duflo and Udry 2004). Food expenditures occur on a regular basis and are typically made from more regular sources of income. However, coffee income is more seasonal. Larger revenues accrue twice a year during or shortly after the two main coffee harvesting seasons. This money is typically not used for regular food expenditures, but is rather spent on clearing bills or making investments into more durable non-food items, such as school uniforms and learning materials. Indeed, participants in our focus group discussions explained that "coffee pays for children's education." This education effect of higher coffee income is further 
reinforced by the fact that the Fairtrade standard restricts child labor, thus reducing the opportunity cost of attending school, as explained above.

The regression results in table 5 confirm that Fairtrade certification increases expenditures on child education significantly, even after controlling for other relevant factors. In columns (1) to (4), the dependent variable is household education expenditures divided by the number of children in primary school age. In columns (5) to (8), household education expenditures are divided by the number of children in primary and secondary school age. In both versions, the Fairtrade effect is positive and highly significant. The coefficient estimate of 0.90 in column (8) of table 5 suggests that Fairtrade certified households spend 146 percent more on child education than noncertified households. 
Table 5: Effects of certification on education expenditure (panel data models)

\begin{tabular}{|c|c|c|c|c|c|c|c|c|}
\hline & \multicolumn{4}{|c|}{ Expenditure per child of primary school age } & \multicolumn{4}{|c|}{ Exp. per child of primary or secondary school age } \\
\hline & (1) & (2) & (3) & (4) & $(5)$ & (6) & (7) & $(8)$ \\
\hline & RE & FE & $\mathrm{RE}$ & FE & $\mathrm{RE}$ & FE & RE & FE \\
\hline Certified (1/0) & $\begin{array}{l}0.58 \\
(0.14)\end{array}$ & $\begin{array}{c}0.73 \\
(0.45)\end{array}$ & & & $\begin{array}{l}0.61 \\
(0.15)\end{array}$ & $\begin{array}{c}0.69 \\
(0.43)\end{array}$ & & \\
\hline Fairtrade $(1 / 0)$ & & & $\begin{array}{l}0.85^{* \pi *} \\
(0.17)\end{array}$ & $\begin{array}{l}1.03^{* * \pi} \\
(0.39)\end{array}$ & & & $\begin{array}{l}0.92^{\text {*** }} \\
(0.17)\end{array}$ & $\begin{array}{l}0.90^{* \pi} \\
(0.36)\end{array}$ \\
\hline Organic $(1 / 0)$ & & & $\begin{array}{c}0.26 \\
(0.17)\end{array}$ & $\begin{array}{c}0.65 \\
(0.56)\end{array}$ & & & $\begin{array}{c}0.23 \\
(0.18)\end{array}$ & $\begin{array}{c}0.63 \\
(0.53)\end{array}$ \\
\hline Year $=2015$ & $\begin{array}{c}0.68^{\text {*** }} \\
(0.12)\end{array}$ & $\begin{array}{c}0.68^{\text {*** }} \\
(0.16)\end{array}$ & $\begin{array}{l}0.65^{* \pi \times} \\
(0.12)\end{array}$ & $\begin{array}{l}0.67^{* \pi *} \\
(0.17)\end{array}$ & $\begin{array}{c}0.64^{* \pi \times} \\
(0.12)\end{array}$ & $\begin{array}{c}0.67^{\pi * \pi} \\
(0.14)\end{array}$ & $\begin{array}{l}0.60^{* \pi * \pi} \\
(0.12)\end{array}$ & $\begin{array}{c}0.67^{* \pi+\pi} \\
(0.15)\end{array}$ \\
\hline No. of children primary school age & $\begin{array}{c}-0.24^{* * *} \\
(0.05)\end{array}$ & $\begin{array}{l}-0.14 \\
(0.09)\end{array}$ & $\begin{array}{c}-0.22^{* *_{*}} \\
(0.05)\end{array}$ & $\begin{array}{l}-0.14^{*} \\
(0.09)\end{array}$ & $\begin{array}{l}-0.03 \\
(0.05)\end{array}$ & $\begin{array}{l}-0.01 \\
(0.08)\end{array}$ & $\begin{array}{l}-0.02 \\
(0.05)\end{array}$ & $\begin{array}{l}-0.01 \\
(0.08)\end{array}$ \\
\hline No. of household members ${ }^{a}$ & $\begin{array}{l}0.18^{\text {*ax }} \\
(0.03)\end{array}$ & $\begin{array}{c}0.08 \\
(0.06)\end{array}$ & $\begin{array}{l}0.19^{* \pi \cdot \pi} \\
(0.03)\end{array}$ & $\begin{array}{c}0.08 \\
(0.06)\end{array}$ & & & & \\
\hline No. of children secondary school age & & & & & $\begin{array}{l}-0.08 \\
(0.06)\end{array}$ & $\begin{array}{c}-0.24^{* *} \\
(0.11)\end{array}$ & $\begin{array}{l}-0.06 \\
(0.06)\end{array}$ & $\begin{array}{c}-0.25^{\text {** }} \\
(0.11)\end{array}$ \\
\hline No. of household members ${ }^{b}$ & & & & & $\begin{array}{c}0.17^{* * \pi} \\
(0.04)\end{array}$ & $\begin{array}{l}0.11^{* \pi \times 4} \\
(0.05)\end{array}$ & $\begin{array}{c}0.18^{\text {* }} \\
(0.04)\end{array}$ & $\begin{array}{l}0.11^{\text {. }} \\
(0.05)\end{array}$ \\
\hline Female headed household (1/0) & $\begin{array}{l}-0.05 \\
(0.19)\end{array}$ & $\begin{array}{l}-1.85 \\
(1.14)\end{array}$ & $\begin{array}{c}0.01 \\
(0.18)\end{array}$ & $\begin{array}{l}-1.84 \\
(1.14)\end{array}$ & $\begin{array}{l}-0.09 \\
(0.20)\end{array}$ & $\begin{array}{l}-1.96 \\
(1.03)\end{array}$ & $\begin{array}{l}-0.04 \\
(0.20)\end{array}$ & $\begin{array}{l}-1.96^{6} \\
(1.03)\end{array}$ \\
\hline Household head schooling (yrs.) & $\begin{array}{c}0.09^{\text {*3. }} \\
(0.02)\end{array}$ & $\begin{array}{l}-0.03 \\
(0.06)\end{array}$ & $\begin{array}{c}0.07^{\text {*3. }} \\
(0.02)\end{array}$ & $\begin{array}{l}-0.03 \\
(0.06)\end{array}$ & $\begin{array}{c}0.09^{* 3 x} \\
(0.02)\end{array}$ & $\begin{array}{l}-0.03 \\
(0.05)\end{array}$ & $\begin{array}{c}0.07^{\text {* }} \\
(0.02)\end{array}$ & $\begin{array}{c}-0.03 \\
(0.05)\end{array}$ \\
\hline Household head age (yrs.) & $\begin{array}{l}-0.00 \\
(0.01)\end{array}$ & $\begin{array}{c}0.00 \\
(0.02)\end{array}$ & $\begin{array}{l}-0.00 \\
(0.01)\end{array}$ & $\begin{array}{c}0.00 \\
(0.02)\end{array}$ & $\begin{array}{l}-0.00 \\
(0.01)\end{array}$ & $\begin{array}{l}-0.03 \\
(0.02)\end{array}$ & $\begin{array}{l}-0.01 \\
(0.01)\end{array}$ & $\begin{array}{l}-0.03 \\
(0.02)\end{array}$ \\
\hline Distance to primary school $(\mathrm{km})$ & $\begin{array}{l}-0.02 \\
(0.06)\end{array}$ & $\begin{array}{l}-0.10 \\
(0.08)\end{array}$ & $\begin{array}{l}-0.01 \\
(0.06)\end{array}$ & $\begin{array}{l}-0.10 \\
(0.08)\end{array}$ & $\begin{array}{l}-0.04 \\
(0.06)\end{array}$ & $\begin{array}{l}-0.10 \\
(0.08)\end{array}$ & $\begin{array}{l}-0.04 \\
(0.06)\end{array}$ & $\begin{array}{c}-0.09 \\
(0.08)\end{array}$ \\
\hline Distance to secondary school $(\mathrm{km})$ & $\begin{array}{l}-0.00 \\
(0.02)\end{array}$ & $\begin{array}{l}-0.00 \\
(0.03)\end{array}$ & $\begin{array}{c}0.00 \\
(0.02)\end{array}$ & $\begin{array}{l}-0.00 \\
(0.03)\end{array}$ & $\begin{array}{l}-0.00 \\
(0.02)\end{array}$ & $\begin{array}{l}-0.02 \\
(0.03)\end{array}$ & $\begin{array}{c}0.00 \\
(0.02)\end{array}$ & $\begin{array}{l}-0.01 \\
(0.03)\end{array}$ \\
\hline Constant & $\begin{array}{l}5.08^{2} \\
(0.34)\end{array}$ & $\begin{array}{l}6.54^{* 2 \pi} \\
(1.57)\end{array}$ & $\begin{array}{l}5.16^{5} \\
(0.35)\end{array}$ & $\begin{array}{l}6.46^{5} \\
(1.57)\end{array}$ & $\begin{array}{c}4.76^{*} \\
(0.39)\end{array}$ & $\begin{array}{l}7.89^{* \pi} \\
(1.42)\end{array}$ & $\begin{array}{c}4.87^{\text {. }} \\
(0.40)\end{array}$ & $\begin{array}{l}7.86^{\text {\% }} \\
(1.42)\end{array}$ \\
\hline No. of observations & 596 & 596 & 596 & 596 & 643 & 643 & 643 & 643 \\
\hline No. of households & 358 & 358 & 358 & 358 & 374 & 374 & 374 & 374 \\
\hline F-value & & $5.85^{\text {*a* }}$ & & $6.73^{\text {क*n }}$ & & $3.65^{\text {करत }}$ & & $3.83^{\text {कin }}$ \\
\hline Wald $\chi^{2}$ & $141.62^{\text {**a }}$ & & $164.59^{\text {*** }}$ & & $107.82^{* * \pi}$ & & $132.59^{\text {*** }}$ & \\
\hline Hausman test $\chi^{2}$ & $18.20^{\text {** }}$ & & $17.43^{*}$ & & $18.76^{* \pi}$ & & $18.05^{*}$ & \\
\hline Sargan-Hansen test $\chi^{2}$ & $16.82^{*}$ & & $16.61^{*}$ & & $17.37^{*}$ & & 16.59 & \\
\hline
\end{tabular}

${ }^{\mathrm{a}}$ Excludes household members of primary school age.

${ }^{\mathrm{b}}$ Excludes household members of primary or secondary school age.

Cluster robust standard errors in parentheses. The dependent variable in all models is the logarithm of expenditure. RE, random effects. FE, fixed effects.

${ }^{*} p<0.1,{ }^{* *} p<0.05,{ }^{* * *} p<0.01$ 
Table 6 shows results from the cross-section models explained in equation (3) with OLS and IV estimators. Columns (1) and (2) use individual education expenditure as dependent variable, whereas in columns (3) and (4) the dependent variable is individual years of schooling.

Table 6: Effects of certification on individual child education (cross-section data models)

\begin{tabular}{|c|c|c|c|c|}
\hline & \multicolumn{2}{|c|}{ Education expenditure (log) } & \multicolumn{2}{|c|}{ Years of schooling } \\
\hline & (1) & (2) & (3) & (4) \\
\hline & OLS & IV & OLS & IV \\
\hline \multirow[t]{2}{*}{ Fairtrade $(1 / 0)$} & $0.72^{* * *}$ & $0.64^{* * *}$ & $0.69^{* * *}$ & $0.66^{* * *}$ \\
\hline & $(0.19)$ & $(0.16)$ & $(0.13)$ & $(0.13)$ \\
\hline \multirow[t]{2}{*}{ Organic $(1 / 0)$} & 0.34 & 0.16 & 0.10 & -0.00 \\
\hline & $(0.22)$ & $(0.25)$ & $(0.15)$ & $(0.16)$ \\
\hline \multirow[t]{2}{*}{ Children primary school age } & $0.13^{* *}$ & $0.13^{* *}$ & $0.11^{* * * * *}$ & $0.11^{\text {*** }}$ \\
\hline & $(0.06)$ & $(0.06)$ & $(0.03)$ & $(0.03)$ \\
\hline \multirow[t]{2}{*}{ Children secondary school age } & 0.02 & 0.02 & -0.02 & -0.01 \\
\hline & $(0.06)$ & $(0.06)$ & $(0.03)$ & $(0.04)$ \\
\hline \multirow[t]{2}{*}{ Household members not school age } & 0.06 & 0.06 & -0.05 & -0.05 \\
\hline & $(0.05)$ & $(0.05)$ & $(0.03)$ & $(0.03)$ \\
\hline \multirow[t]{2}{*}{ Female headed household (1/0) } & 0.17 & 0.18 & $0.25^{*}$ & $0.26^{*}$ \\
\hline & $(0.23)$ & $(0.21)$ & $(0.13)$ & $(0.13)$ \\
\hline \multirow[t]{2}{*}{ Household head schooling (yrs.) } & $0.07^{* * *}$ & $0.07^{* * *}$ & $0.06^{* * * * *}$ & $0.05^{* * *}$ \\
\hline & $(0.02)$ & $(0.02)$ & $(0.02)$ & $(0.02)$ \\
\hline \multirow[t]{2}{*}{ Household head age (yrs.) } & $-0.01^{*}$ & -0.01 & -0.00 & -0.00 \\
\hline & $(0.01)$ & $(0.01)$ & $(0.01)$ & $(0.01)$ \\
\hline \multirow[t]{2}{*}{ Distance to primary school $(\mathrm{km})$} & -0.03 & -0.04 & 0.03 & 0.03 \\
\hline & $(0.08)$ & $(0.08)$ & $(0.04)$ & $(0.04)$ \\
\hline \multirow[t]{2}{*}{ Distance to secondary school (km) } & 0.01 & 0.01 & 0.01 & 0.01 \\
\hline & $(0.02)$ & $(0.02)$ & $(0.01)$ & $(0.02)$ \\
\hline \multirow[t]{2}{*}{ Female child (1/0) } & $0.24^{*}$ & $0.23^{* *}$ & $0.59^{* * * *}$ & $0.57^{* * *}$ \\
\hline & $(0.13)$ & $(0.11)$ & $(0.09)$ & $(0.09)$ \\
\hline \multirow[t]{2}{*}{ Age of child (yrs.) } & $0.06^{* * *}$ & $0.06^{* * *}$ & $0.63^{* * * * 3}$ & $0.63^{* * *}$ \\
\hline & $(0.02)$ & $(0.02)$ & $(0.01)$ & $(0.01)$ \\
\hline \multirow[t]{2}{*}{ Constant } & $10.39^{* * * *}$ & $10.43^{* * *}$ & & \\
\hline & $(0.42)$ & $(0.54)$ & & \\
\hline No. of observations & 1122 & 1122 & 1120 & 1120 \\
\hline No. of households (clusters) ${ }^{a}$ & 329 & 329 & 329 & 329 \\
\hline F-value & $4.16^{* * *}$ & & & \\
\hline Wald $\chi^{2}$ & & $72.91^{* * *}$ & $1137.09^{* * *}$ & $2210.51^{* * *}$ \\
\hline Hausman test $\chi^{2}$ & $24.46^{* *}$ & & 4.80 & \\
\hline Wald $\left(\chi^{2}\right)$ first stage Fairtrade ${ }^{b}$ & & $64.76^{* * *}$ & & $64.76^{* * *}$ \\
\hline Wald $\left(\chi^{2}\right)$ first stage Organic ${ }^{b}$ & & $37.58^{* * *}$ & & $37.58^{* * *}$ \\
\hline Goodness-of-fit $\chi^{2}$ & & & 91.31 & \\
\hline
\end{tabular}

The estimates confirm that Fairtrade certification significantly increases investments in child education. Furthermore, controlling for other factors, Fairtrade increases child 
schooling by 0.66 years. These results are similar to earlier findings on Fairtrade in other countries by Gitter et al. (2012), Arnould, Plastina, and Ball (2009), and Becchetti, Castriota, and Michetti (2013).

Results in table 6 further suggest that there is no discrimination against girls in child education. On the contrary, households spend about 30 percent more on girls' than boy's education. And, on average, girls stay 0.57 years longer in school than boys. These effects are independent of certification status and may possibly be explained by higher opportunity costs of attending school for boys and young men (Gitter et al. 2012). In any case, these effects are welcome from a women empowerment perspective.

\subsubsection{Organic certified households}

Unlike Fairtrade, for Organic certified households we do not find significant effects on education expenditures or on years of schooling (tables 5 and 6). This is consistent with results in table 4 that revealed significant positive effects of Organic certification on food expenditures, but not non-food expenditures. We explain likely mechanisms for these effects below.

As discussed, food expenditures capture the value of all food items consumed by the household, including subsistence production. Home-produced foods are important components of diets in smallholder farm households, and this is especially true for Organic certified households in our sample. Figure 1 shows that Organic certified households are less specialized in coffee production (panel A) and have more diversified farm production systems (panel C) than Fairtrade and non-certified households. This can be explained by the principles of the Organic standard that explicitly promote measures to increase production diversity. For instance, the large majority of Organic certified households grow legumes to improve and maintain soil fertility (table 4). Higher production diversity and a stronger focus on subsistence consumption tend to be associated with foregone benefits from specialization and lower cash incomes (Sibhatu, Krishna, and Qaim 2015). This can also explain the lower nonfood expenditures observed in Organic certified households.

We now analyze whether the higher food expenditures caused by Organic certification are also associated with improved household diets and nutrition. Regression results in table 7 confirm that Organic certification is associated with lower levels of energy and micronutrient deficiencies. The marginal effects suggest that Organic certification 
reduces the likelihood of energy deficiency by 19 percentage points, and the likelihood of iron, zinc, and vitamin A deficiencies by 12-24 percentage points.

However, the probit models in table 7 cannot be estimated with a FE estimator. In table A3 in the appendix we show alternative results, using a linear probability model and comparing RE and FE estimates. The FE coefficients for Organic certification are insignificant in all models except for vitamin A. Thus, while there is a clear positive association between Organic certification and dietary quality, we only have weak evidence to prove causality. The positive vitamin A effect of Organic certification is promising, because vitamin A deficiency is often particularly difficult to control without specific interventions. The reason is that the income elasticity of vitamin A consumption tends to be lower than that for many other micronutrients (Ecker and Qaim 2011). For Fairtrade certification, all diet and nutrition effects in tables 7 and A3 are statistically insignificant. 
Table 7: Effects of certification on energy and micronutrient deficiency (probit panel data models)

\begin{tabular}{|c|c|c|c|c|c|c|c|c|}
\hline & \multicolumn{2}{|c|}{ Energy deficiency } & \multicolumn{2}{|c|}{ Iron deficiency } & \multicolumn{2}{|c|}{ Zink deficiency } & \multicolumn{2}{|c|}{ Vitamin A deficiency } \\
\hline & (1) & $(2)$ & $(3)$ & (4) & $(5)$ & $(6)$ & $(7)$ & $(8)$ \\
\hline & $\mathrm{RE}$ & RE & RE & RE & RE & RE & RE & RE \\
\hline Certified (1/0) & $\begin{array}{l}-0.06 \\
(0.04)\end{array}$ & & $\begin{array}{l}-0.07^{* * *} \\
(0.04)\end{array}$ & & $\begin{array}{l}-0.04 \\
(0.03)\end{array}$ & & $\begin{array}{l}-0.07 \\
(0.04)\end{array}$ & \\
\hline Fairtrade $(1 / 0)$ & & $\begin{array}{c}0.05 \\
(0.04)\end{array}$ & & $\begin{array}{c}0.05 \\
(0.04)\end{array}$ & & $\begin{array}{c}0.03 \\
(0.04)\end{array}$ & & $\begin{array}{c}0.06 \\
(0.05)\end{array}$ \\
\hline Organic $(1 / 0)$ & & $\begin{array}{c}-0.19^{* * *} \\
(0.05)\end{array}$ & & $\begin{array}{c}-0.22^{* * *} \\
(0.05)\end{array}$ & & $\begin{array}{c}-0.12^{* * * *} \\
(0.04)\end{array}$ & & $\begin{array}{c}-0.24^{* * *} \\
(0.05)\end{array}$ \\
\hline Year $=2015$ & $\begin{array}{l}-0.04 \\
(0.04)\end{array}$ & $\begin{array}{l}-0.05 \\
(0.03)\end{array}$ & $\begin{array}{c}0.09^{* * * *} \\
(0.04)\end{array}$ & $\begin{array}{l}0.08^{* * *} \\
(0.04)\end{array}$ & $\begin{array}{c}0.04 \\
(0.03)\end{array}$ & $\begin{array}{c}0.03 \\
(0.03)\end{array}$ & $\begin{array}{l}0.14^{* * * *} \\
(0.04)\end{array}$ & $\begin{array}{c}0.12^{* * * *} \\
(0.04)\end{array}$ \\
\hline Household size (AE) & $\begin{array}{c}0.07^{* * *} \\
(0.02)\end{array}$ & $\begin{array}{c}0.07^{* * * *} \\
(0.02)\end{array}$ & $\begin{array}{l}0.07^{* * * *} \\
(0.02)\end{array}$ & $\begin{array}{c}0.07^{* * * *} \\
(0.02)\end{array}$ & $\begin{array}{c}0.07^{* * * *} \\
(0.02)\end{array}$ & $\begin{array}{c}0.06^{* * * *} \\
(0.02)\end{array}$ & $\begin{array}{c}0.02 \\
(0.02)\end{array}$ & $\begin{array}{c}0.02 \\
(0.02)\end{array}$ \\
\hline Household size (AE) squared & $\begin{array}{l}-0.00 \\
(0.00)\end{array}$ & $\begin{array}{l}-0.00 \\
(0.00)\end{array}$ & $\begin{array}{l}-0.00 \\
(0.00)\end{array}$ & $\begin{array}{l}-0.00 \\
(0.00)\end{array}$ & $\begin{array}{l}-0.00 \\
(0.00)\end{array}$ & $\begin{array}{l}-0.00 \\
(0.00)\end{array}$ & $\begin{array}{l}-0.00 \\
(0.00)\end{array}$ & $\begin{array}{l}-0.00 \\
(0.00)\end{array}$ \\
\hline Female headed household (1/0) & $\begin{array}{l}-0.04 \\
(0.05)\end{array}$ & $\begin{array}{l}-0.03 \\
(0.05)\end{array}$ & $\begin{array}{l}-0.05 \\
(0.04)\end{array}$ & $\begin{array}{l}-0.03 \\
(0.04)\end{array}$ & $\begin{array}{c}0.01 \\
(0.04)\end{array}$ & $\begin{array}{c}0.02 \\
(0.04)\end{array}$ & $\begin{array}{l}-0.04 \\
(0.06)\end{array}$ & $\begin{array}{l}-0.02 \\
(0.05)\end{array}$ \\
\hline Household head schooling (yrs.) & $\begin{array}{l}0.01^{*} \\
(0.01)\end{array}$ & $\begin{array}{c}0.00 \\
(0.01)\end{array}$ & $\begin{array}{c}0.02^{* * * *} \\
(0.00)\end{array}$ & $\begin{array}{l}0.01^{* * *} \\
(0.01)\end{array}$ & $\begin{array}{l}0.01^{*} \\
(0.00)\end{array}$ & $\begin{array}{c}0.00 \\
(0.00)\end{array}$ & $\begin{array}{l}0.01^{* * *} \\
(0.01)\end{array}$ & $\begin{array}{c}0.00 \\
(0.01)\end{array}$ \\
\hline Household head age (yrs.) & $\begin{array}{l}-0.00 \\
(0.00)\end{array}$ & $\begin{array}{l}-0.00 \\
(0.00)\end{array}$ & $\begin{array}{l}-0.00 \\
(0.00)\end{array}$ & $\begin{array}{l}-0.00^{*} \\
(0.00)\end{array}$ & $\begin{array}{c}-0.00^{* *} \\
(0.00)\end{array}$ & $\begin{array}{c}-0.00^{* * * *} \\
(0.00)\end{array}$ & $\begin{array}{c}0.00 \\
(0.00)\end{array}$ & $\begin{array}{c}0.00 \\
(0.00)\end{array}$ \\
\hline Distance to all-weather road $(\mathrm{km})$ & $\begin{array}{l}-0.00 \\
(0.00)\end{array}$ & $\begin{array}{l}-0.00 \\
(0.00)\end{array}$ & $\begin{array}{l}-0.00 \\
(0.00)\end{array}$ & $\begin{array}{l}-0.00 \\
(0.00)\end{array}$ & $\begin{array}{c}-0.01^{\text {***** }} \\
(0.00)\end{array}$ & $\begin{array}{c}-0.00^{* * *} \\
(0.00)\end{array}$ & $\begin{array}{l}-0.00^{*} \\
(0.00)\end{array}$ & $\begin{array}{l}-0.00 \\
(0.00)\end{array}$ \\
\hline No. of observations & 740 & 740 & 740 & 740 & 740 & 740 & 740 & 740 \\
\hline No. of households ${ }^{\text {a }}$ & 409 & 409 & 409 & 409 & 409 & 409 & 409 & 409 \\
\hline Wald $\chi^{2}$ & $47.25^{* * *}$ & $59.97^{* * *}$ & $59.12^{* * *}$ & $62.72^{* * *}$ & $63.66^{* * *}$ & $70.82^{* * *}$ & $25.84^{* * *}$ & $41.05^{* * *}$ \\
\hline
\end{tabular}




\subsection{Conclusion}

The empirical evidence on impacts of sustainability standards in the small farm sector is growing. We have contributed to this literature by comparing the effects of two popular sustainability standards, Organic and Fairtrade, on household welfare in Uganda. Unlike most previous research that built on cross-section data, we have used panel data that are more suitable to reduce selection bias. Moreover, we have looked at various indicators of household welfare, including consumption expenditures, child education, and nutrition.

In line with previous research, we have shown that Fairtrade and Organic certification have positive welfare effects in terms of total consumption (Chiputwa, Spielman, and Qaim 2015). However, in terms of the other welfare indicators we found remarkable differences. Fairtrade increases non-food expenditures and child education, whereas Organic increases food expenditures and to some extent nutrition. We found no effects of Fairtrade on food expenditures and nutrition, and no effects of Organic on non-food expenditures and child education. These differences in impacts were explained with differences in the principles of each standard and different types of services offered to certified households. Such insights are not only relevant for producers, but also for consumers, and other actors along certified value chains.

Our results suggest that food standards can be a tool to promote sustainability goals in the small farm sector. On the one hand, standards can contribute to higher household incomes. On the other hand - through trainings and recommended practices - standards also have the potential to raise awareness on issues such as education, nutrition, or gender equality. A precondition is that such social issues are specifically addressed in certification schemes. Fairtrade includes specific rules and activities to reduce child labor and increase education, but not to improve nutrition. Given widespread dietary deficiencies among smallholder farm households, the design of sustainability standards should place more emphasis on nutrition. Certification agencies could instigate participating farmer organizations to offer training on nutrition - as is already common practice for other topics such as environmental stewardship.

More generally, our results show that economic gains from agricultural development interventions are not necessarily reflected in terms of other welfare dimensions, such as child education, household diets, and nutrition. Analyzing welfare effects beyond purely economic indicators is of particular importance for achieving the United Nation's 
Sustainable Development Goals (SDGs). The analytical approaches proposed and used in this study can be further refined and possibly used also for the evaluation of other types of rural development projects and policies.

A few limitations of our study should be pointed out that could be addressed in followup research. First, the impacts of a food standard do not only depend on the principles of the standard itself, but also on the specific local conditions. Hence, the concrete results from our study in Uganda should not be generalized. Second, our study builds on a short panel with relatively little variation in the treatment variables. Longer panels with more variation would be useful to also look at impact dynamics and to further reduce endogeneity issues. Finally, although we looked at different areas of household welfare, not all dimensions of potential interest were actually captured. For instance, gender equality is one dimension that was not included here, but that would be relevant to include in future research. 


\subsection{Appendix}

Table A1: Predicated probabilities of participation in Fairtrade and Organic certification

\begin{tabular}{lcc}
\hline & $(1)$ & $(2)$ \\
& Fairtrade & Organic \\
\hline Distance to Fairtrade organization building $(\mathrm{km})$ & $-0.02^{* * *}$ & \\
& $(0.00)$ & $-0.00^{* * *}$ \\
Altitude $(\mathrm{m})$ & & $(0.00)$ \\
& & 0.02 \\
Number of children primary school age & -0.01 & $(0.02)$ \\
& $(0.01)$ & $0.05^{* * *}$ \\
Number of children secondary school age & 0.00 & $(0.02)$ \\
& $(0.01)$ & 0.01 \\
Number of household members not school age & 0.01 & $(0.01)$ \\
& $(0.00)$ & 0.00 \\
Female headed household (1/0) & 0.02 & $(0.05)$ \\
Household head schooling (yrs.) & $(0.03)$ & -0.00 \\
& 0.00 & $(0.01)$ \\
Household head age (yrs.) & $(0.00)$ & $0.00^{* *}$ \\
Distance to primary school (km) & 0.00 & $(0.00)$ \\
& $(0.00)$ & -0.01 \\
Distance to secondary school (km) & 0.00 & $(0.02)$ \\
Female (1/0) & $(0.01)$ & -0.00 \\
Age (yrs.) & -0.00 & $(0.01)$ \\
& $(0.00)$ & -0.01 \\
No. of individuals & -0.02 & $(0.02)$ \\
Wald $\chi^{2}$ & $(0.01)$ & 0.00 \\
Averaseholds (clusters) & $0.00^{*}$ & $(0.00)$ \\
\hline & $(0.00)$ & 1122 \\
& 1122 & $46.93^{* * *}$ \\
\hline
\end{tabular}

Average marginal effects are shown with cluster robust standard errors in parentheses.

${ }^{*} p<0.1,{ }^{* *} p<0.05,{ }^{* * *} p<0.01$ 
Table A2: Regressions for subsample of non-certified households

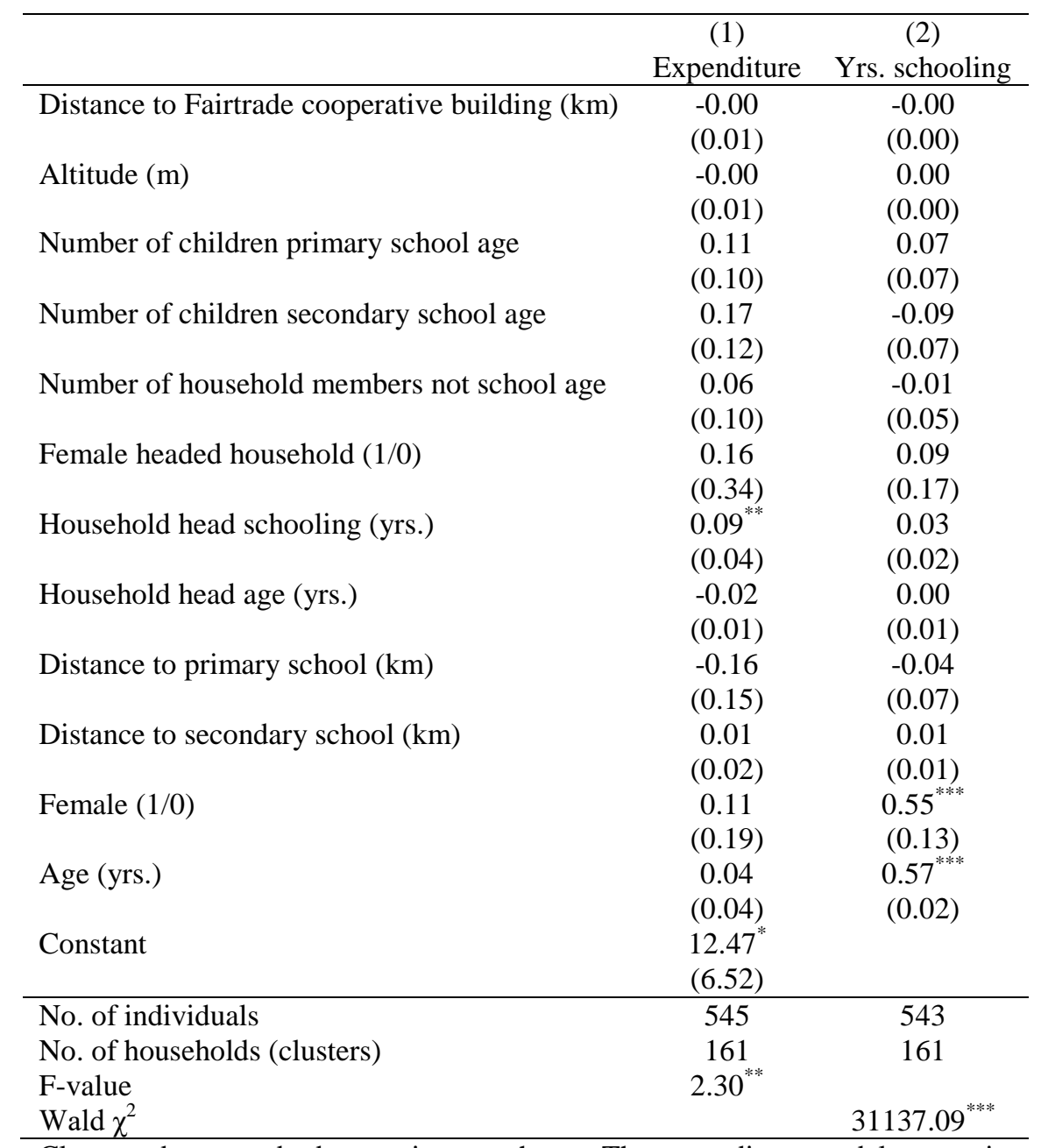

Cluster robust standard errors in parentheses. The expenditure model was estimated with OLS. The years of schooling model with a Poisson, for which marginal effects are shown.

${ }^{*} p<0.1,{ }^{* * *} p<0.05,{ }^{* * *} p<0.01$ 
Table A3: Effects of certification on energy and micronutrient deficiency (linear probability models)

\begin{tabular}{|c|c|c|c|c|c|c|c|c|}
\hline & \multicolumn{2}{|c|}{ Kcal } & \multicolumn{2}{|c|}{ Iron } & \multicolumn{2}{|c|}{ Zinc } & \multicolumn{2}{|c|}{ Vitamin A } \\
\hline & (1) & (2) & (3) & (4) & (5) & (6) & (7) & (8) \\
\hline & $\mathrm{RE}$ & FE & RE & FE & $\mathrm{RE}$ & FE & $\mathrm{RE}$ & $\mathrm{FE}$ \\
\hline \multirow[t]{2}{*}{ Fairtrade $(1 / 0)$} & 0.05 & -0.04 & 0.06 & 0.11 & 0.03 & -0.16 & 0.05 & 0.04 \\
\hline & $(0.04)$ & $(0.14)$ & $(0.05)$ & $(0.15)$ & $(0.03)$ & $(0.10)$ & $(0.04)$ & $(0.15)$ \\
\hline \multirow[t]{2}{*}{ Organic $(1 / 0)$} & $-0.16^{* * *}$ & 0.06 & $-0.21^{* * *}$ & 0.15 & $-0.13^{* * *}$ & -0.07 & $-0.22^{* * *}$ & $-0.31^{* *}$ \\
\hline & $(0.04)$ & $(0.10)$ & $(0.04)$ & $(0.11)$ & $(0.04)$ & $(0.12)$ & $(0.05)$ & $(0.13)$ \\
\hline \multirow[t]{2}{*}{ Year $=2015$} & -0.04 & -0.02 & $0.11^{* * * *}$ & $0.15^{* * * *}$ & 0.03 & 0.03 & $0.09^{* *}$ & $0.08^{* *}$ \\
\hline & $(0.03)$ & $(0.04)$ & $(0.03)$ & $(0.04)$ & $(0.03)$ & $(0.03)$ & $(0.04)$ & $(0.04)$ \\
\hline \multirow[t]{2}{*}{ Household size (AE) } & $0.06^{* * * *}$ & $0.09^{* * * * *}$ & 0.02 & $0.09^{* * *}$ & $0.09^{* * * *}$ & $0.13^{* * *}$ & $0.07^{* * * *}$ & $0.12^{* * *}$ \\
\hline & $(0.02)$ & $(0.04)$ & $(0.02)$ & $(0.04)$ & $(0.02)$ & $(0.03)$ & $(0.02)$ & $(0.04)$ \\
\hline \multirow[t]{2}{*}{ Household size (AE) squared } & -0.00 & -0.00 & -0.00 & $-0.00^{*}$ & $-0.00^{* * *}$ & $-0.00^{* *}$ & -0.00 & -0.00 \\
\hline & $(0.00)$ & $(0.00)$ & $(0.00)$ & $(0.00)$ & $(0.00)$ & $(0.00)$ & $(0.00)$ & $(0.00)$ \\
\hline \multirow[t]{2}{*}{ Female headed household (1/0) } & -0.02 & $0.38^{* * *}$ & -0.01 & 0.30 & 0.02 & $0.37^{* * * *}$ & -0.03 & $0.53^{* * * *}$ \\
\hline & $(0.04)$ & $(0.16)$ & $(0.05)$ & $(0.19)$ & $(0.04)$ & $(0.10)$ & $(0.04)$ & $(0.15)$ \\
\hline \multirow[t]{2}{*}{ Household head schooling (yrs.) } & 0.00 & -0.00 & 0.00 & 0.00 & 0.00 & 0.00 & $0.01^{* *}$ & -0.01 \\
\hline & $(0.00)$ & $(0.02)$ & $(0.01)$ & $(0.02)$ & $(0.00)$ & $(0.01)$ & $(0.01)$ & $(0.02)$ \\
\hline \multirow[t]{2}{*}{ Household head age (yrs.) } & -0.00 & 0.00 & 0.00 & 0.00 & $-0.00^{* * * *}$ & 0.00 & -0.00 & $0.01^{* *}$ \\
\hline & $(0.00)$ & $(0.00)$ & $(0.00)$ & $(0.01)$ & $(0.00)$ & $(0.00)$ & $(0.00)$ & $(0.00)$ \\
\hline \multirow[t]{2}{*}{ Distance to all-weather road $(\mathrm{km})$} & -0.00 & 0.00 & -0.00 & 0.00 & $-0.00^{* * *}$ & $-0.01^{* *}$ & -0.00 & -0.00 \\
\hline & $(0.00)$ & $(0.00)$ & $(0.00)$ & $(0.00)$ & $(0.00)$ & $(0.00)$ & $(0.00)$ & $(0.00)$ \\
\hline \multirow[t]{2}{*}{ Constant } & 0.16 & -0.37 & $0.24^{*}$ & -0.39 & $0.65^{* * * *}$ & 0.13 & $0.24^{* * *}$ & $-0.74^{* *}$ \\
\hline & $(0.11)$ & $(0.26)$ & $(0.12)$ & $(0.36)$ & $(0.09)$ & $(0.27)$ & $(0.10)$ & $(0.33)$ \\
\hline Observations & 740 & 740 & 740 & 740 & 740 & 740 & 740 & 740 \\
\hline No. of households & 409 & 409 & 409 & 409 & 409 & 409 & 409 & 409 \\
\hline F-value & & $6.10^{* * *}$ & & $3.44^{* * *}$ & & $5.46^{* * *}$ & & $6.64^{* * *}$ \\
\hline Wald $\chi^{2}$ & $113.52^{* * *}$ & & $57.60^{* * *}$ & & $134.90^{* * *}$ & & $160.93^{* * *}$ & \\
\hline Hausman test $\chi^{2}$ & $18.89^{* *}$ & & & & $26.83^{* * *}$ & & $30.03^{* * *}$ & \\
\hline Sargan-Hansen test $\chi^{2}$ & $18.73^{* *}$ & & & & $26.28^{* * *}$ & & $30.00^{* * *}$ & \\
\hline
\end{tabular}

Cluster robust standard errors in parentheses.

${ }^{*} p<0.1,{ }^{* * *} p<0.05,{ }^{* * *} p<0.01$ 


\title{
3 Can private food standards promote gender equality in the small farm sector?
}

\begin{abstract}
Agricultural commercialization can lift subsistence farmers in developing countries out of poverty - but can have adverse effects on gender equality. Here, we explore whether sustainability standards could serve as a vehicle to promote gender equality in smallholder cash crop production. We focus on two standards that explicitly address gender issues, namely Fairtrade and UTZ. This is the first quantitative study to analyze gendered implications of sustainability standards in depth. Our analysis is based on cross-sectional, gender-disaggregated data from certified and non-certified smallholder coffee producers in Uganda. We employ entropy balancing to control for observed heterogeneity between certified and non-certified farmers. To reduce possible bias from unobserved heterogeneity, we use estimates on farmers' willingness to accept (WTA) sustainability standards as a conditioning variable in reweighting the data. We find that sustainability standards increase household assets - including women's assets. In maleheaded households, standards also affect the distribution of wealth within households. While in non-certified households most assets are owned by the male household head alone, in certified households most assets are owned jointly by the male head and female spouse. Certified farmers also have better access to agricultural extension, irrespective of their gender. Yet, sustainability standards have no effect on women's access to financial services. We conclude that sustainability standards may not completely eliminate gender disparities, but can contribute towards this goal.
\end{abstract}

Keywords: cash crops, certification, gender, sustainability standards, women's empowerment 


\subsection{Introduction}

The United Nation's Sustainable Development Goals reinforce the importance of gender equality and women's empowerment for poverty reduction and food and nutrition security (UN 2016). Yet, achieving gender equality remains a challenge, especially in rural areas of developing countries, where poverty is particularity persistent (FAO 2011).

Agricultural commercialization is seen as an important strategy to lift subsistence farmers out of poverty (Maertens and Swinnen 2009; Rao and Qaim 2011). Yet, the evidence suggests that agricultural commercialization can have adverse effects on women's empowerment and gender equality. Women farmers may find it more difficult to participate in modern value chains, given gender disparities in terms of access to land and agricultural inputs and services (Maertens and Swinnen 2012; Quisumbing et al. 2015). Such gender disparities are not specific to any particular region, but hamper agricultural productivity and women's access to high value markets across the developing world (FAO 2011). Further, social norms and gender roles may limit women's engagement in cash crop production and marketing (Njuki et al. 2011; Handschuch and Wollni 2015). Therefore, cash crops are sometimes labelled 'men's crops' (Orr et al. 2016). Several studies also show that women may lose control over agricultural income from food crops, when these become profitable (von Braun and Kennedy 1994; Fischer and Qaim 2012; Chege, Andersson, and Qaim 2015). This is not only problematic from a women's empowerment perspective, but also from a broader welfare perspective (Doss 2013; Malapit and Quisumbing 2015). For instance, the evidence shows that women are likely to make expenditure decisions that promote child nutrition and wellbeing (Hoddinott and Haddad 1995).

Here, we explore if private food standards could serve to mitigate potentially negative effects of agricultural commercialization on gender equality. Private food standards are gaining in importance in global food chains - including for crops produced by smallholder farmers in developing countries (Maertens and Swinnen 2009; Lee, Gereffi, and Beauvais 2012). Private food standards cover a wide range of issues, such as food safety and quality, welfare, labor conditions, and environmental stewardship. Here, we focus on two popular standards that are aimed at promoting sustainability, namely UTZ and Fairtrade. UTZ and Fairtrade, among other sustainability standards, include specific components to promote women's empowerment and gender equality (Fairtrade International 2009; UTZ 2015c). For instance, certified farmer organizations have to comply with non-discrimination 
policies. Further, they are encouraged to organize workshops to raise awareness on gender equality; to implement special programs tailored to women farmers' needs; and to promote women farmers' participation in regular agricultural training sessions. However, not all sustainability standards include such gender components. Therefore, understanding whether such gender components prove effective in promoting gender equality could be useful.

A growing body of literature has analyzed welfare effects of sustainability standards on smallholder farm households, with mixed results (e.g. Bolwig, Gibbon, and Jones 2009; Jones and Gibbon 2011; Kleemann, Abdulai, and Buss 2014; Chiputwa, Spielman, and Qaim 2015; Chiputwa and Qaim 2016; Ibanez and Blackman 2016; van Rijsbergen et al. 2016; Mitiku et al. 2017). However, these studies typically focus on the household as the unit of observation. Thus, issues of intra-household distribution of costs and benefits cannot be analyzed. Further, it remains unclear whether sustainability standards can serve to promote gender equality and women's empowerment (Terstappen, Hanson, and McLaughlin 2013). A few quantitative studies look at gender issues (Ruben and Fort 2012; Chiputwa and Qaim 2016), but without analyzing gendered implications in detail - or based on individual-level data. Further, some qualitative studies describe experiences of female workers in the certified agro-processing or handicraft sector (e.g. Hutchens 2010; Bonnan-White, Hightower, and Issa 2013). Some qualitative studies (e.g. Lyon 2008; Lyon, Bezaury, and Mutersbaugh 2010; Bacon 2010; Loconto 2015) also explore gender aspects of food standards in the small farm sector (see chapter 3.2).

To our knowledge, this is the first quantitative study to analyze gendered implications of sustainability standards in depth. Our objectives are to analyze (1) if sustainability standards benefit women and men in male-headed households, (2) if costs and benefits associated with the adoption of sustainability standards are equally distributed within maleheaded households, and (3) if female-headed households benefit from sustainability standards. Thus, we analyze impacts on both female spouses and female household heads, which is seldom done. Our analysis is based on gender-disaggregated survey data from certified and non-certified smallholder coffee producers in Uganda. Some of the sample households are UTZ or Fairtrade certified; others are not certified. We analyze the effect of sustainability standards on various outcome variables, capturing different areas of empowerment within households and beyond. Outcome variables include gendered asset ownership, participation in trainings and farmer group meetings, access to financial services, and time allocation. For the econometric analysis, we use entropy balancing 
(Hainmueller 2012), allowing us to control for observed heterogeneity. To reduce possible bias from unobserved heterogeneity, we use estimates of farmers' willingness to accept (WTA) sustainability standards as a conditioning variable in reweighting the data.

\subsection{Sustainability standards, gender components, and possible effects on gender equality}

The following literature review motivates our empirical strategy. We hypothesize that sustainability standards can contribute to promoting gender equality, provided that gender issues are accounted for in the standard. In the following, we summarize how gender issues are addressed in the standards we focus on, namely Fairtrade and UTZ. Afterwards, we discuss how standards may affect different areas of empowerment, based on previous qualitative findings on gender and standards and related literature. We focus on the following areas of empowerment: economic empowerment, division of labor, social capital, and access to services. The importance of considering these areas is described below. We take up these areas of empowerment in our empirical strategy and in presenting our results.

\subsubsection{Gender components of Fairtrade and UTZ}

In this study, we analyze the gendered effects of Fairtrade ${ }^{11}$ and UTZ standards. We take these two standards as examples of sustainability standards that include gender components. We do not disaggregate between Fairtrade and UTZ in our analysis, because these two standards are very comparable, especially regarding their gender approach. UTZ and Fairtrade highlight their commitment to promote gender equality on their homepages and in several reports (Fairtrade International 2009, 2011b; UTZ 2015b, 2015c). Fairtrade has developed a gender strategy (Fairtrade International 2009) which is aimed at mainstreaming gender along Fairtrade value chains - from standard setting to implementation. UTZ recommends the use of its 'gender check list' to promote gender equality. Further, UTZ is piloting gender-sensitive approaches to auditing, which includes the training of auditors on gender issues (UTZ 2015c).

\footnotetext{
${ }^{11}$ We refer to Fairtrade standards set by Fairtrade International (Fairtrade International 2011b).
} 
Fairtrade and UTZ standards also specify a range of mandatory and suggested measures on gender equality. The specific goal of these measures is to raise awareness on gender issues and to strengthen women's position within households, farmer organizations, and communities (Fairtrade International 2009, 2011b; UTZ 2015b, 2015c). Measures can be broadly grouped into three categories. The first set of measures refers to non-discrimination policies. UTZ and Fairtrade farmer organizations have to respect the principle of nondiscrimination in recruiting and paying staff. Further, women employees have the right to maternity leave. Sexual harassment must not be accepted (Fairtrade International 2011b; UTZ 2015a, 2015c). UTZ additionally encourages farmer organizations to ensure equal representation of disadvantaged groups (incl. women) among their staff (e.g. extension officers or farmer organization leadership) (UTZ 2015a, 2015c).

The second set of measures relates to special gender programs. The implementation is voluntary. Fairtrade and UTZ farmer organizations are encouraged to implement workshops or trainings on gender equality, targeting both women and men. Further, the introduction of special programs or trainings tailored to women farmers' needs is also encouraged (Fairtrade International 2009, 2011b; UTZ 2015c). Fairtrade farmer organizations sometimes use parts of the Fairtrade premium ${ }^{12}$ for such programs (Fairtrade International 2011b). The third set of measures is aimed at increasing women's participation in regular (agricultural) trainings, group meetings, and other activities implemented by certified farmer organizations. To promote this goal, UTZ farmer organizations have to take specific measures: women have to be informed about training sessions; trainings have to be held at times feasible for women; and attendance of women and men during trainings has to be documented using participant lists (UTZ 2015a).

\subsubsection{Possible effects on economic empowerment}

Individual economic endowment (e.g. income, control over cash income, or asset ownership) is a key driver of women's empowerment (Kabeer 1999; Doss 2013; Johnson et al. 2016). Women who are employed (e.g. in the agro-processing sector) or who sell their own crops generate their own income, contributing to their economic empowerment (Maertens and Swinnen 2012). However, women's role in cash crop production and

\footnotetext{
${ }^{12}$ The Fairtrade premium is paid to Fairtrade farmer organizations and is to be invested in development projects (Fairtrade International 2011b).
} 
marketing may be limited - and cash crops and the income generated from sales are often controlled by men (Njuki et al. 2011). Most certified crops produced by smallholder farmers are traditional cash crops. Drawing from previous studies, we hypothesize that sustainability standards may contribute to women's economic empowerment (1) by improving women's access to markets and income or (2) by increasing women's control over income from cash crop production.

Some studies suggest that standards and certification requirements can promote women's role in cash crop production and their access to markets and income. For instance, Lyon, Bezaury, and Mutersbaugh (2010) found that, in Mesoamerica, Fairtrade-Organic standards promoted women's involvement in coffee production and marketing. Many women registered as farm operators and farmer group members. This development was attributable to high rates of men migration and certification requirements. Specifically, registered farm operators were required to be present during announced and unannounced certification audits, promoting women's role as registered farm operators. Kloos and Renaud (2014) found that access to certified markets increased women's involvement in cotton production in Benin. Cotton production and handling of chemical pesticides and synthetic fertilizers was locally perceived as a male task. Under Organic, the use of these inputs is prohibited, which increased social acceptance of women's Organic cotton production. Further, certified cotton was produced on the least fertile plots. Similarly, looking at West Africa, Bassett (2010) showed that women's role in cotton production increased due to the introduction of standards. Households aimed at accessing both certified and non-certified marketing channels. Male household heads continued to manage the largest share of the cotton area conventionally. Their spouses became members of the certified producer organization and produced certified cotton on a certain share of the area.

While standards and context-specific factors can increase women's access to markets and income, this does not always hold. Several studies suggest that women's role in marketing and supply chains does not change with the introduction of standards. Thus, some studies conclude that economic gains from standards and certification are captured by men (Lyon 2008; Bolwig 2012; Sen 2014; Loconto 2015). Chiputwa and Qaim (2016) also found that standards do not challenge men's role in coffee marketing. However, they showed that sustainability standards can nevertheless increase women's control over coffee revenues. They attributed this trend to non-discrimination policies and workshops on gender equality introduced by certified farmer organizations. 


\subsubsection{Possible effects on the division of labor and women's workload}

Agricultural tasks and responsibilities are often gender-specific in the small farm sector. Therefore, agricultural interventions may affect women's and men's workload in different ways (Doss 2001; Quisumbing et al. 2015). Women are often strongly involved in laborintensive activities (e.g. weeding, harvesting, washing, and sorting in the case of coffee). Sustainability standards and related certification requirements on farming and post-harvest management tend to increase labor demand for these female activities (Lyon, Bezaury, and Mutersbaugh 2010; Bolwig 2012). Especially Organic standards, which prohibit the use of chemical pesticides, may increase women's workload (Bolwig 2012). However, whether women's workload increases as a result of standards and related certification requirements also depends on whether capital is reinvested in equipment that can reduce demand for manual labor (Lyon, Bezaury, and Mutersbaugh 2010; Bolwig 2012). It is also known that household members may renegotiate the division of agricultural tasks and responsibilities when circumstances change (Orr et al. 2016). Theoretically, standards could have the same effect. However, previous studies suggests that sustainability standards neither change the gendered division of labor nor decision making in agriculture (Bolwig 2012; Ruben and Fort 2012).

\subsubsection{Possible effects on social capital}

Social capital and collective action are key to linking women farmers to markets and for their empowerment (Fischer and Qaim 2012; Handschuch and Wollni 2015). In the small farm sector, farmer organizations are one important platform to enhance social capital. Yet, women farmers are often excluded from farmer organizations (Doss 2001; Fischer and Qaim 2012). Sustainability standards may increase women's participation in farmer organizations, especially if measures are taken to promote women's participation. Yet, the evidence on whether sustainability standards deliver on this goal is mixed. Some qualitative studies suggest that sustainability standards fail to increase women's representation in farmer organizations (Lyon 2008; Sen 2014). Figures provided by Fairtrade International support that men often dominate certified farmer organizations. About 20 percent of the registered members in Fairtrade certified farmer organizations are women (Fairtrade International 2009). This may be problematic because being a registered member is often a precondition to influence decisions, e.g., on services to be provided by certified farmer organizations (Lyon 2008). Even in organizations with a higher share of female members, 
men often occupy leadership positions (Bacon 2010) and may disapprove women's active participation and ideas (Lyon 2008; Sen 2014). Additionally, women's participation in meetings may be passive or low (Lyon 2008; Sen 2014). Other studies highlight advances in terms of women participation in farmer groups and decision making, due to standards (Elder, Zerriffi, and Le Billon 2012). Further, women-led initiatives may emerge within certified farmer organizations (Bacon 2010; Elder, Zerriffi, and Le Billon 2012). There are also examples of women-only cooperatives, such as ‘café feminino' (Lyon 2008).

\subsubsection{Possible effects on the access to services}

Women farmers are often disadvantaged in terms of access to agricultural and financial services. Such gender disparities can explain low adoption of agricultural technologies, lower yields, and poor access to markets among women (Doss 2001; FAO 2011). Sustainability standards may improve women's access to such services. Certified farmer organizations usually offer agricultural trainings and other services to their members. Especially if specific measures are taken, women's participation in such trainings may increase. Women's access to information may also improve if female extension officers are employed by certified farmer organizations.

\subsection{Research context and household survey}

\subsubsection{Survey and data}

Our analysis builds on a household survey conducted in 2015 with small-scale coffee producers in Uganda. We employed a two-stage sampling strategy: First, we purposively selected two coffee farmer organizations, located in central Uganda. One of these organizations is Fairtrade certified, the other is UTZ certified. However, not all members of these farmer organizations are actually certified. For farm households, certification is a voluntary decision. Second, we randomly selected certified and non-certified households, based on complete membership lists provided by each farmer organization. 
We collected data at the household and individual level. ${ }^{13}$ In male-headed and femaleheaded households, we interviewed the male or female household head. ${ }^{14}$ Additionally, in male-headed households we interviewed female spouses. ${ }^{15}$ Interviews were conducted separately with male and female spouses. Table 8 provides an overview of the sample households by certification status. In total, we conducted interviews with 346 households. Among them 174 are certified and 172 are not certified. Within these households, we received individual responses from 548 individuals, including 233 male household heads, 244 female spouses, and 71 female household heads. ${ }^{16}$ In Table 8 the number of respondents, from whom we received individual responses, is displayed in parentheses.

Table 8: Overview of sample households and individuals by certification status

\begin{tabular}{rccc}
\hline & Certified & Non-certified & Total \\
\hline Total no. of households & 174 & 172 & 346 \\
Total no. of individuals & $311(278)$ & $303(270)$ & $614(548)$ \\
& & & \\
Male-headed households & 137 & 131 & 268 \\
Male household heads & $137(119)$ & $131(114)$ & $268(233)$ \\
Female spouses & $137(126)$ & $131(118)$ & $268(244)$ \\
& & & 78 \\
Female-headed households & 37 & $41(38)$ & $78(71)$ \\
Female household heads & $37(33)$ & &
\end{tabular}

Interviews were conducted by local enumerators, who were trained and supervised by the researchers. The questionnaire covers farm, household, and contextual characteristics. We also collected detailed information on household assets and individual asset ownership. The questionnaire further encompasses detailed questions on time allocation and participation in training sessions and other group actives.

\footnotetext{
13 When analyzing intra-household dynamics, two approaches are commonly used (Doss et al. 2014). Following the first, all adult household members are considered. Following, the second, only couples (household heads and their spouses) are considered. Here, we follow the second approach.

${ }^{14}$ In a few cases, the household head was not available for interviews. In such cases, we interviewed the female spouse.

${ }^{15}$ Some households in our sample $(\mathrm{N}=5)$ are polygamous. In such cases, we asked the household head, who was interviewed first, to identify the female spouse with the greatest decision-making power.

${ }^{16}$ Female household heads include widowed, single, divorced, or separated women. Married women who live alone (e.g. because their spouse migrated) are also considered female household heads.
} 


\subsubsection{Gender policies implemented by farmer organizations}

As discussed, Fairtrade and UTZ standards specify a range of mandatory and voluntary measures to promote gender equality (see gender components of Fairtrade and UTZ). As the set of gender measures may thus vary by farmer organization, we briefly summarize the measures implemented by the sample farmer organizations. Both farmer organizations meet standards on non-discrimination. For instance, both women and men are hired as extension and certification officers as well as for administrative positions. Further, workshops are organized to raise awareness on gender equality. The specific purpose of these workshops is help spouses and other household members to work as a team and to appreciate the work done by other household members. Additionally, both farmer organizations provide agricultural services to their members, including credits and agricultural trainings. Theoretically, any farmer could join training sessions on gender equality or farming, irrespective of her certification status. However, certified farmers are particularly encouraged to participate. Training sessions and regular interactions between farmers and certification officers serve to ensure that certified farmers understand and comply with certification requirements. Compliance of certified members is pivotal, because otherwise the farmer organization may lose its certificate.

\subsection{Empirical strategy}

Our goal is to analyze how sustainability standards affect female household heads (in female-headed households) and female spouses and male household heads (in male-headed households). We consider different areas of empowerment, following the categories defined earlier (i.e. economic empowerment, division of labor and workload, social capital, and access to services). For each category, we selected different outcome variables. After describing each outcome variable, we explain our econometric approach.

\subsubsection{Selection and measurement of outcome variables}

We use asset ownership as an indicator of women's economic empowerment and bargaining power. Asset ownership is a suitable proxy for these outcomes, because it determines individuals' options and livelihood opportunities (Doss et al. 2014; Quisumbing et al. 2015; Johnson et al. 2016). For instance, assets serve a as collateral when seeking credit. However, assets and related opportunities are often distributed unequally within 
households - and often favor men (Deere and Doss 2006; Doss et al. 2014). Looking at individual asset ownership allows us to assess how wealth is distributed within households - and if standards change the distribution of wealth within households. ${ }^{17}$ In this study, we look at the current market value of assets in 1000 Ugandan Shilling (UGX). Assets include durable consumption goods and productive assets, such as furniture, means of transportation, electronic devices, agricultural equipment, and livestock. We do not include assets such as land and housing, because these assets were probably acquired prior to certification. ${ }^{18}$ Consequently, including these assets would not allow us to detect changes in empowerment - and thus to test causality between standards and empowerment.

One challenge with using asset ownership as an empowerment indicator is that assets can be held jointly or individually (Quisumbing et al. 2015). In male-headed households, male household heads often have more rights than their spouses over assets that are held jointly (e.g. when it comes to selling assets) (Johnson et al. 2016). We address this challenge by analyzing individual asset ownership and joint asset ownership separately. In male-headed households, we look at (1) the value of total household assets, (2) the value of assets owned by the male household head, (3) the value of assets owned by the female spouse, and (4) joint ownership. In addition to the absolute value, we also consider relative figures (e.g. individual ownership of the male head as a percentage of total household assets). For female-headed households, we focus on the value of assets owned by the female household head (in absolute terms and as a percentage of total household assets).

To analyze how standards may affect the workload of women and men, we follow Alkire et al. (2013) and use a 24 hour recall that captures all activities pursued during the last day. These data are based on individual-level responses. We aggregated hours each person spent on farm, off-farm, and domestic work. As proposed by Alkire et al. (2013) we also asked for peoples' satisfaction regarding their time available for leisure activities. This variable can take values from one (very unsatisfied) to five (very satisfied).

\footnotetext{
${ }^{17}$ Other studies have used control over income as a proxy for women's empowerment (e.g. Chiputwa and Qaim 2016). Using control over income, it is not possible to assess how wealth is distributed within households. Therefore, we prefer looking at asset ownership. Asset ownership also reflects longer term economic development and expenditure decisions.

${ }^{18}$ Both farmer organizations adopted UTZ/Fairtrade standards around the year 2007.
} 
To proxy social capital, we asked respondents if they participated in farmer group meetings during the past twelve months. We also asked if they hold a leadership position in any group (e.g. famer, women's, religious group, etc.)

To analyze whether sustainability standards improve access to agricultural services, we asked respondents if they interacted with an extension officer; and if they participated in field days and agricultural trainings during the past twelve months. Agricultural trainings refer to sessions, demonstrations, and workshops on soil fertility, pest management, and coffee quality improvement. Regarding access to financial services, we asked respondents if they have a personal savings account, if they use mobile money, and if they are a member of a savings group.

\subsubsection{Econometric approach}

To assess the impact of sustainability standards on the outcomes described above, we compare certified against non-certified farmers. Specifically, we compare (1) male household heads in certified and non-certified households, (2) female spouses in certified and non-certified households, and (3) female household heads in certified and non-certified households. As we use observational data, we have to account for the fact that certified and non-certified farmers may differ in terms of observed and unobserved factors. If such factors are correlated with both the treatment (sustainability standards) and the outcomes (variables specified above), our estimation will be biased. To reduce such bias, we use entropy balancing (Hainmueller 2012). Entropy balancing belongs to the family of weighting and matching approaches, such as propensity score matching (PSM) and inverse probability weighting (IPW). Weighting and matching approaches can be used to address imbalances in the distribution of covariates among the treatment and control group. The idea is that when such imbalances are eliminated, the treatment assignment becomes ignorable and the treatment effect can be calculated.

Entropy balancing is a novel approach to covariate balancing, but has been employed for impact evaluation in the field of unemployment and health (Marcus 2013), policy interventions and development (Huang and Yeh 2014; Neuenkirch and Neumeier 2016), and education and earnings (Freier, Schumann, and Siedler 2015). Entropy balancing calculates weights for each untreated individual such that differences in the distribution of covariates between treatment and control group are eliminated. Technically speaking, this 
is a minimization problem, subject to the balancing and non-negativity constraint. Entropy balancing has two advantages over more established methods, such as PSM. First, simultaneously balancing all covariates can prove challenging using PSM (Hainmueller 2012). Low levels of covariate balancing are avoided using entropy balancing. Second, entropy balancing uses information from all observations, because no observation is given a weight of zero.

To obtain entropy weights, the researcher first has to select conditioning variables, i.e., variables that are accounted for in reweighting control group observations. All variables that may simultaneously affect the treatment assignment and the outcome should be included. We condition on a rich set of covariates, such as age, education, religion, and farm and contextual characteristics. In the second step, weights obtained through entropy balancing are used to calculate the mean difference in outcomes, or average treatment effect (ATT). Here, we use OLS regressions for continuous outcome variables (e.g. value of assets) and probit regressions for binary outcome variables (e.g. participation in trainings and access to financial services). In these regressions, we include the certification status as a binary treatment variable. As data are reweighted (i.e. balanced), control variables are not required in theses regressions.

The shortcoming of entropy balancing - and other weighting and matching approaches - is that it controls for observed heterogeneity only. In the presence of unobserved heterogeneity, treatment estimates will be biased. To reduce bias from unobserved confounders, we include estimates on farmers' willingness to accept (WTA) sustainability standards as a conditioning variable in reweighting the data. A similar approach was employed by Verhofstadt and Maertens (2014), using PSM. Estimates on farmers' WTA sustainability standards were obtained from a choice experiment carried out with the same farm households (Meemken, Veettil, and Qaim 2016). The choice experiment was used to analyze farmers' preferences for sustainability standards in general and for specific attributes of sustainability standards in particular. Attributes included in the choice experiment are (1) the price premium, (2) provision of agricultural extension, (3) gender policies, (4) requirements on coffee quality, (5) handling of chemical pesticides, and (6) record keeping. WTA estimates are obtained by dividing the coefficient of each attribute by the negative of the price coefficient. Aggregating WTA estimates of all attributes, we obtained farmers' overall WTA sustainability standards. Thus, our WTA variable is a good indicator for farmers' attitudes towards sustainability standards. This variable is useful for 
our analysis, because farmers' attitudes towards sustainability standards are likely correlated with a range of factors that may simultaneously influence farmers' decision to enter certification and our outcome variables. Many of such factors are usually difficult to measure, for instance farmers' openness towards new farming practices, learning ability, or motivation. By including WTA estimates we have a suitable proxy variable for such unobserved confounders, thereby reducing bias due to unobserved heterogeneity.

As a robustness check, we also use inverse probability weighting (IPW) (Hirano, Imbens, and Ridder 2003; Wooldridge 2007), instead of entropy weighting. The IPW estimator first calculates the treatment probability (i.e. the probability of entering certification in our case). Then, the inverse of the treatment probability is used as a weight in estimating the mean outcome of both the treatment and control group. Finally, the mean difference (i.e. treatment effect) is calculated, using the reweighted data. As IPW uses the inverse of the treatment probability, it assigns more weight to observations that receive an unlikely treatment. For instance, larger weights are given to those observations that are among the treated - although their treatment probability is low. The idea of IPW is to better account for all possible outcomes. Pirracchio, Resche-Rigon, and Chevret (2012) show that IPW yields unbiased treatment estimates, even when the sample size is as small as 40 . However, similar to other matching and weighting methods, treatment estimates will only be unbiased when all confounders are included as conditioning variables (i.e. in estimating the treatment probability). We use the same conditioning variables as for entropy balancing, including our WTA estimates. 


\subsection{Results}

We start the analysis by looking at the factors influencing the probability of adopting sustainability standards. Afterwards, we look at the effect of sustainability standards on economic empowerment. Then, we focus on the impact on the division of labor, social capital, and access to services. In each table we show descriptive statistics and average treatment effects (ATT).

\subsubsection{Treatment probability}

In table 9 we look at the factors affecting the probability of adopting sustainability standards. We estimated separate models for male-headed households (column 1) and female-headed households (column 2). We also calculated treatment probabilities at the individual level (i.e. for male household heads, female spouses, and female household heads). This table can be found in the appendix (table A4). We find that various farm, household, and contextual characteristics influence the likelihood of adopting standards. For instance, households with older and better educated heads are more likely to adopt standards (table 9). Further, Muslim households are less likely to adopt sustainability standards. Location-specific factors (e.g. altitude or remoteness) also affect the treatment probability. These factors may not only affect the treatment probability, but also outcomes. As explained (see econometric approach), we use entropy balancing to address such imbalances in the distribution of covariates. 
Table 9: Treatment probabilities (household level)

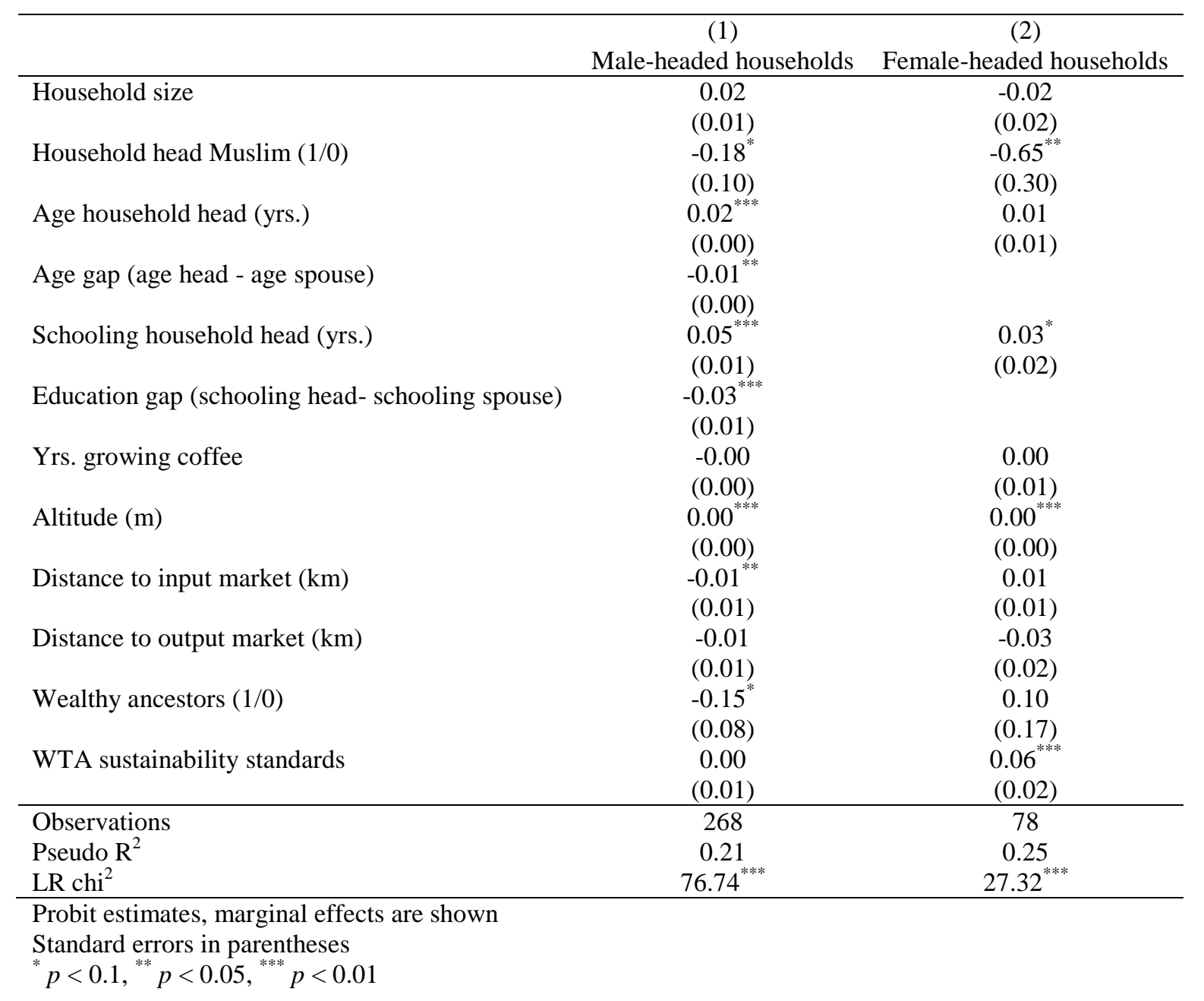

\subsubsection{Economic empowerment}

Figure 2 provides an overview of the distribution of wealth within male-headed households. Differentiating by certification status, we show the average value of assets (in 1000 UGX) owned by male heads, female spouses, or jointly. Generally, female spouses own few assets. Most assets are owned jointly or by male heads. Women's most valuable assets are livestock. Men's most valuable assets are means of transportation and livestock. Figure 2 also suggests that certified households own more assets than non-certified households. Further, the distribution of wealth seems to differ in certified and non-certified households. While in non-certified households the largest share of assets is owned by male heads alone, 
the largest share of assets in certified households is owned jointly by female spouses and male heads. Using entropy balancing, we assess if these differences are attributable to the adoption of standards.

Figure 2: Asset ownership (in 1000 UGX) in male-headed households by category and certification status

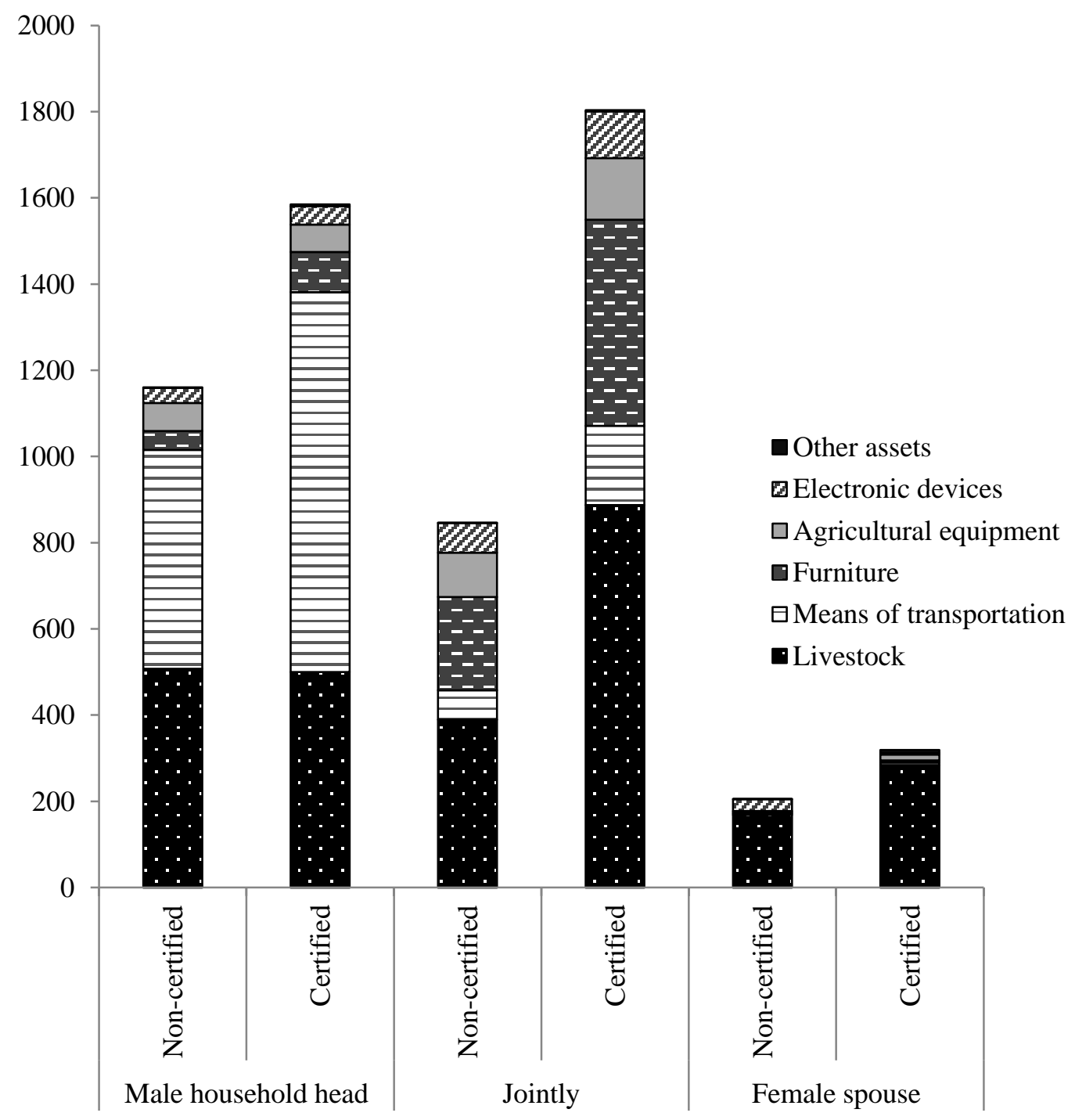

Regression results confirm descriptive statistics (see table 10). In male-headed households, sustainability standards increase household assets by 1162,940 UGX or by about 46 percent (column 4). The largest increase (912,630 UGX) is found for joint asset ownership. Yet, 
standards also have a significant and economically relevant effect on female spouses' assets (i.e. an increase of 146,780 UGX). The coefficient for male household heads' assets is positive but not significant (column 4). Despite this absolute increase, standards reduce the share of household assets owned by male heads alone. Consequently, standards increase the share of assets owned jointly.

Table 10: Asset ownership (in 1000 UGX)

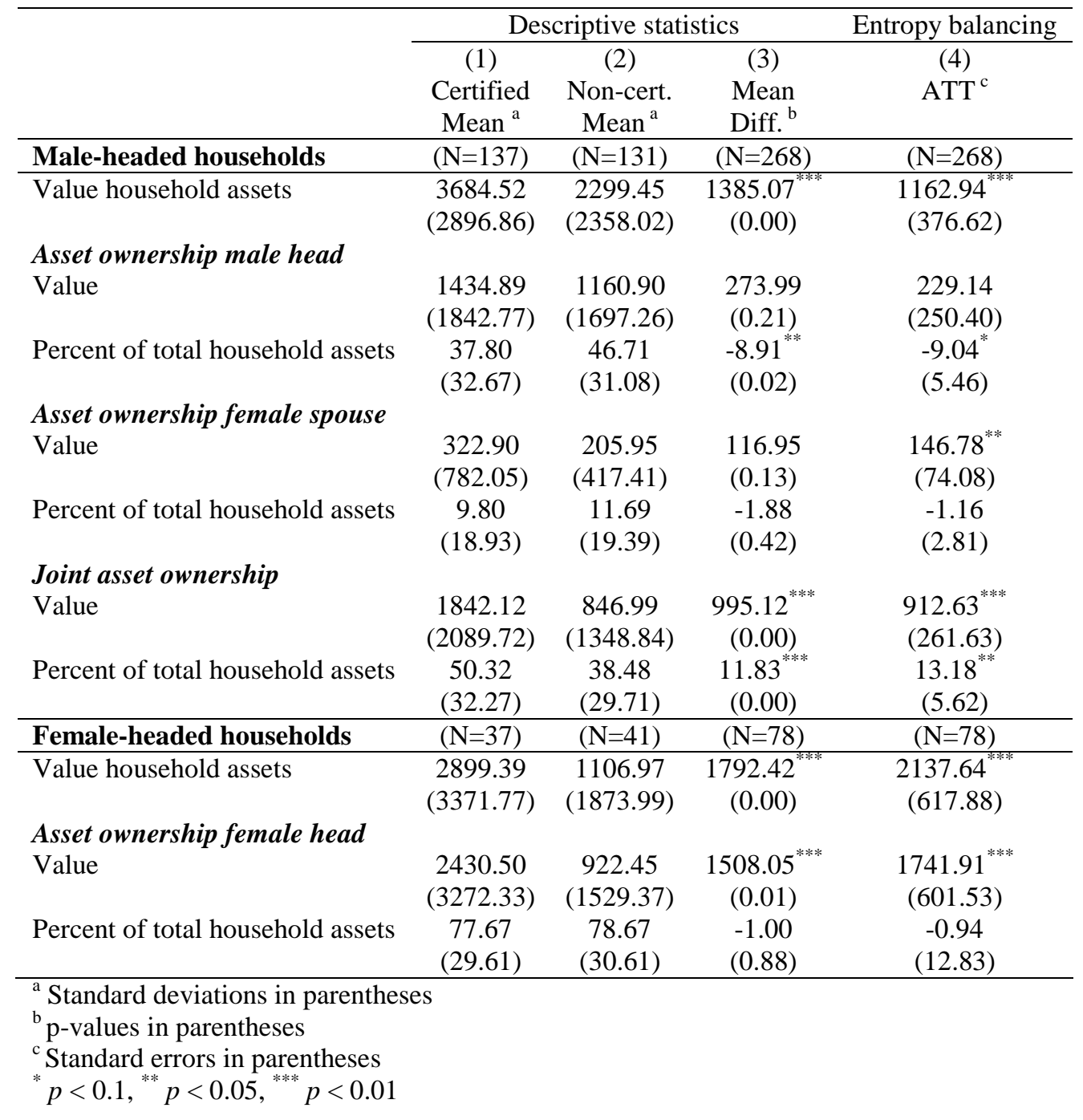

In the lower part of table 10, we focus on female-headed households. Sustainability standards increase the value of total household assets by 2137,640 or 280 percent (column 4). The largest share of household assets (over 70 percent) is owned by female heads. Thus, 
in female-headed households, sustainability standards increase overall household assets and women's individual assets almost to the same extent.

As coffee is the main income generating activity for most sample households, the increase in household wealth among certified households is likely related to higher coffee revenues. Coffee revenues are predominantly controlled by household heads. This holds for both male-headed and female-headed households. Specifically, in 92 percent of female-headed and in 67 percent of male-headed households, coffee revenues are controlled by the household head alone. The redistribution of wealth in certified, male-headed households may be attributable to female spouses' improved control over coffee incomes. To explore this possible pathway, we examine who controls coffee and other crop revenues in maleheaded households. ${ }^{19}$ Figure 3 provides an overview of the crops that are commonly produced and sold by sample households. Differentiating by crop and by certification status, figure 3 shows whether revenues are controlled by male household heads, female spouses, or jointly. We find that coffee revenues - irrespective of the certification status are controlled by male household heads in most male-headed households. Revenues from most other crops are controlled jointly or by female spouses alone. This is not surprising because most of these crops are food crops.

Figure 3 also suggests that, among certified households, revenues are less likely controlled by the male household head alone. Consequently, female spouses in certified households have greater control over crop revenues than their counterparts in non-certified households. This applies not only for coffee, as previously shown by Chiputwa and Qaim (2016), but for all crops.

19 We focus on male-headed households for this overview, because female heads in female-headed households usually control coffee revenues anyways. 
Figure 3: Who controls crop revenues in male-headed households? (Percent of households)

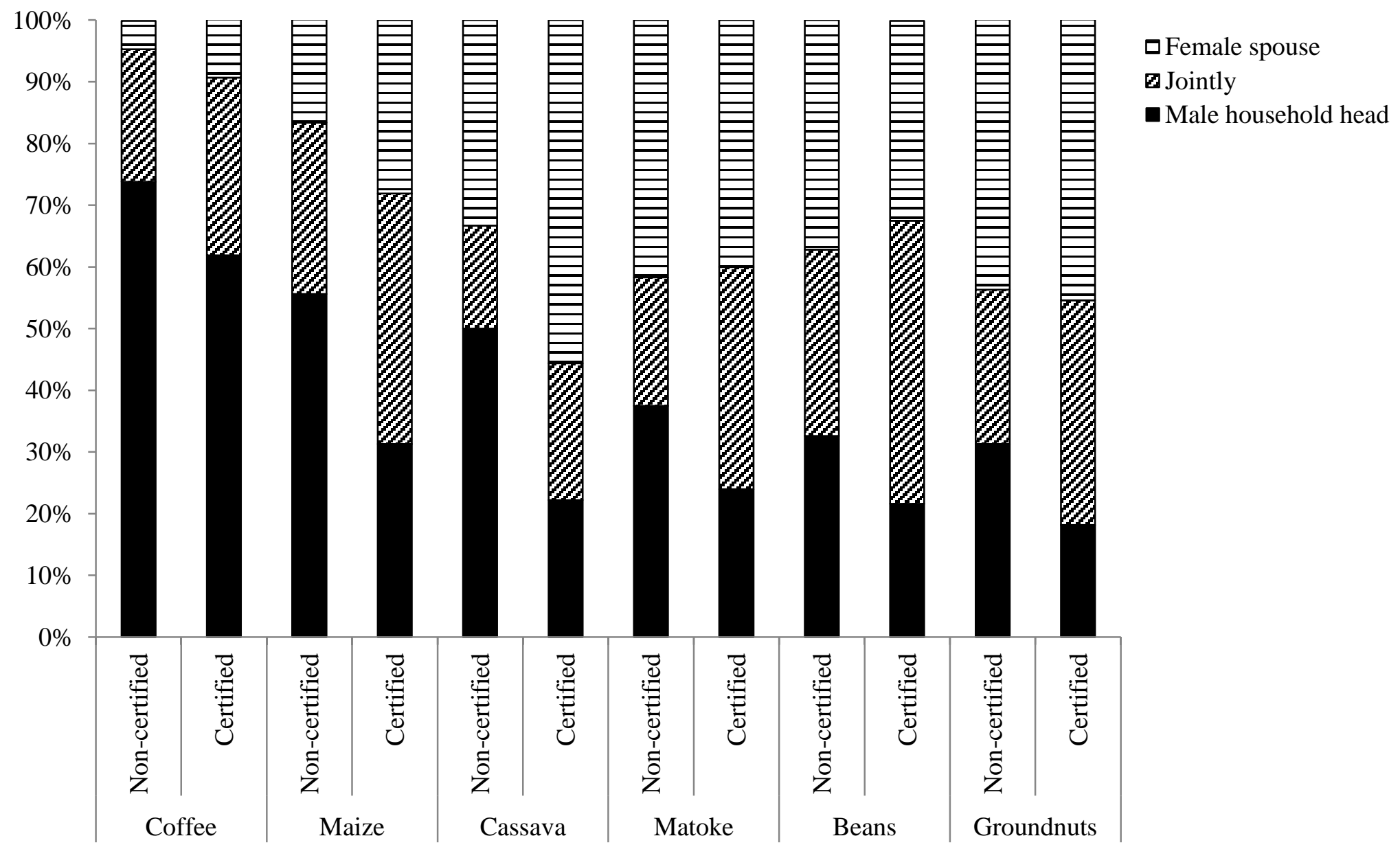

Note: Includes only male-headed households that produce and sell these crops (coffee: 346 households, maize: 134 households, cassava: 21 households, matoke: 72 households, beans: 79 households, groundnuts: 27 households). 


\subsubsection{Division of labor within households and workload}

Figure 4 displays tasks in coffee production in male-headed households by certification status. We find that men control production decisions and marketing in most households - irrespective of the certification status. Further, in both certified and non-certified households, harvesting is a joint activity, undertaken by spouses and other household members. The only difference between certified and non-certified households is observed with respect to control over coffee revenues, as discussed above (see economic empowerment).

Figure 4: Tasks in coffee production in male-headed households by certification status (percent of households)

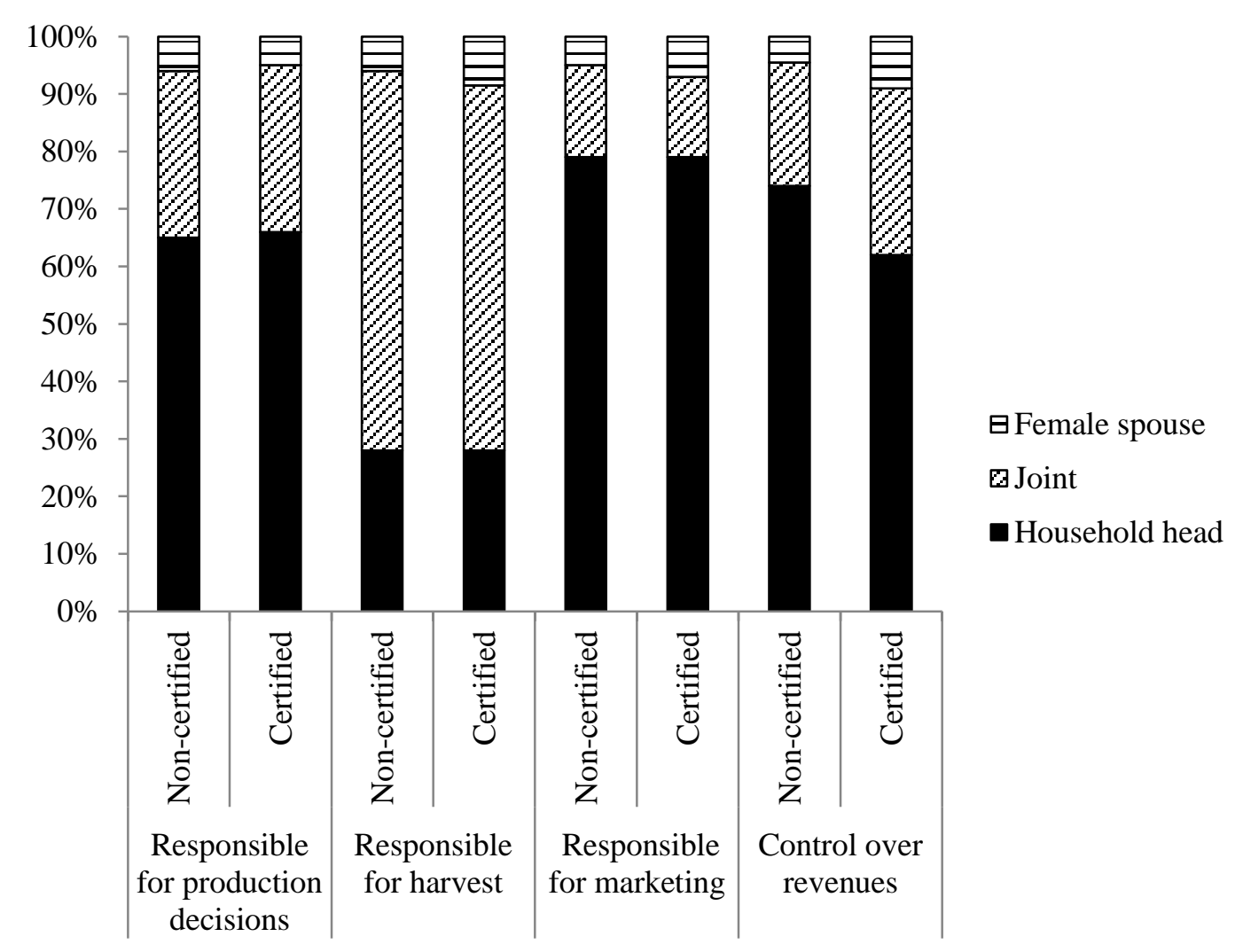

In table 11, we focus on women's and men's workload. Female spouses work on average about 10 hours per day - and about 90 minutes more than their husbands (column 1-2). Further, women in certified (male-headed and female-headed) households spend more time on farm, off-farm, and domestic work than their non-certified counterparts. However, these differences are small and neither significant before 
entropy balancing (column 3) nor after entropy balancing (column 4). Table 11 also suggests that female spouses are more satisfied with their time available for leisure activities than their husbands, despite a higher workload (column 1-2). Yet, sustainability standards have no effect on the level of satisfaction in male-headed households (column 4).

Table 11: Workload and social capital

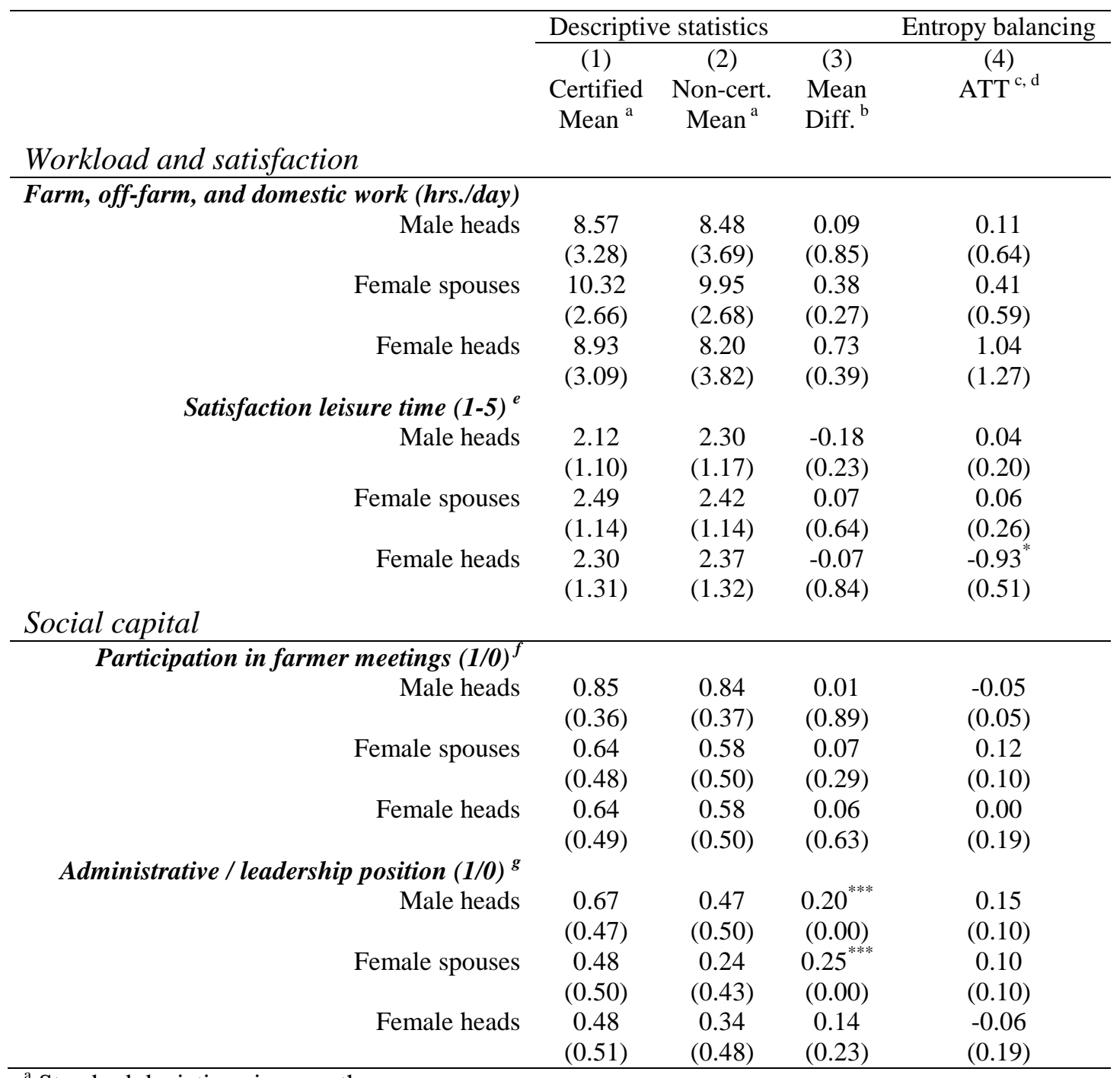

${ }^{\mathrm{a}}$ Standard deviations in parentheses

${ }^{\mathrm{b}} \mathrm{p}$-values in parentheses

${ }^{\mathrm{c}}$ Standard errors in parentheses

${ }^{\mathrm{d}}$ Probit regressions for social capital variables. Marginal effects are shown.

e The variable can take 5 values. One represents the lowest level (low satisfaction); five the highest level (high satisfaction).

${ }^{\mathrm{f}}$ During the past 12 months

${ }^{\mathrm{g}}$ Includes leadership positions in farmer, religious, women's, governmental, help, and trade groups

${ }^{*} p<0.1,{ }^{* *} p<0.05,{ }^{* * * *} p<0.01$ 


\subsubsection{Social capital}

In the lower part of table 11 we look at social capital. Male household heads are more likely to attend farmer group meetings than female spouses and female household heads (column 1-2). Sustainability standards do not change this pattern: they do not increase the probability that individuals participate in farmer group meetings (column 4). Table 4 also suggests that certified farmers are actively involved in group activities. Specifically, 48 percent of women and 67 percent of men in certified households hold administrative or leadership positions (column 1). Yet, differences between certified and non-certified individuals are insignificant after entropy balancing (column 4).

\subsubsection{Access to extension and financial services}

In table 12, we focus on agricultural services. About 48-76 percent of male household heads in certified households interact with extension officers and participate in field days (column 1). Had these men not entered certification, they would be 29-42 percentage points less likely to access such services (column 4). Certified female household heads are also likely to access agricultural information. Indeed, sustainability standards increase the likelihood that female household heads interact with extension offers or participate in training sessions by 54-59 percentage points. In contrast, sustainability standards have a smaller effect on female spouses' access to agricultural information. Standards increase the likelihood that female spouses interact with extension officers (by 31 percentage points), participate in field days (by 17 percentage points), and attend training sessions on coffee quality (by 21 percentage points). Yet, certified female spouses are as unlikely to attend training sessions on soil fertility and pest management as their non-certified counterparts (column 4).

Regarding financial services (lower part of table 12), we find that such services are especially accessed by male household heads. Sustainability standards further improve access to financial services among men (column 4), but have no effect on women's access to financial services. 
Table 12: Access to extension and financial services

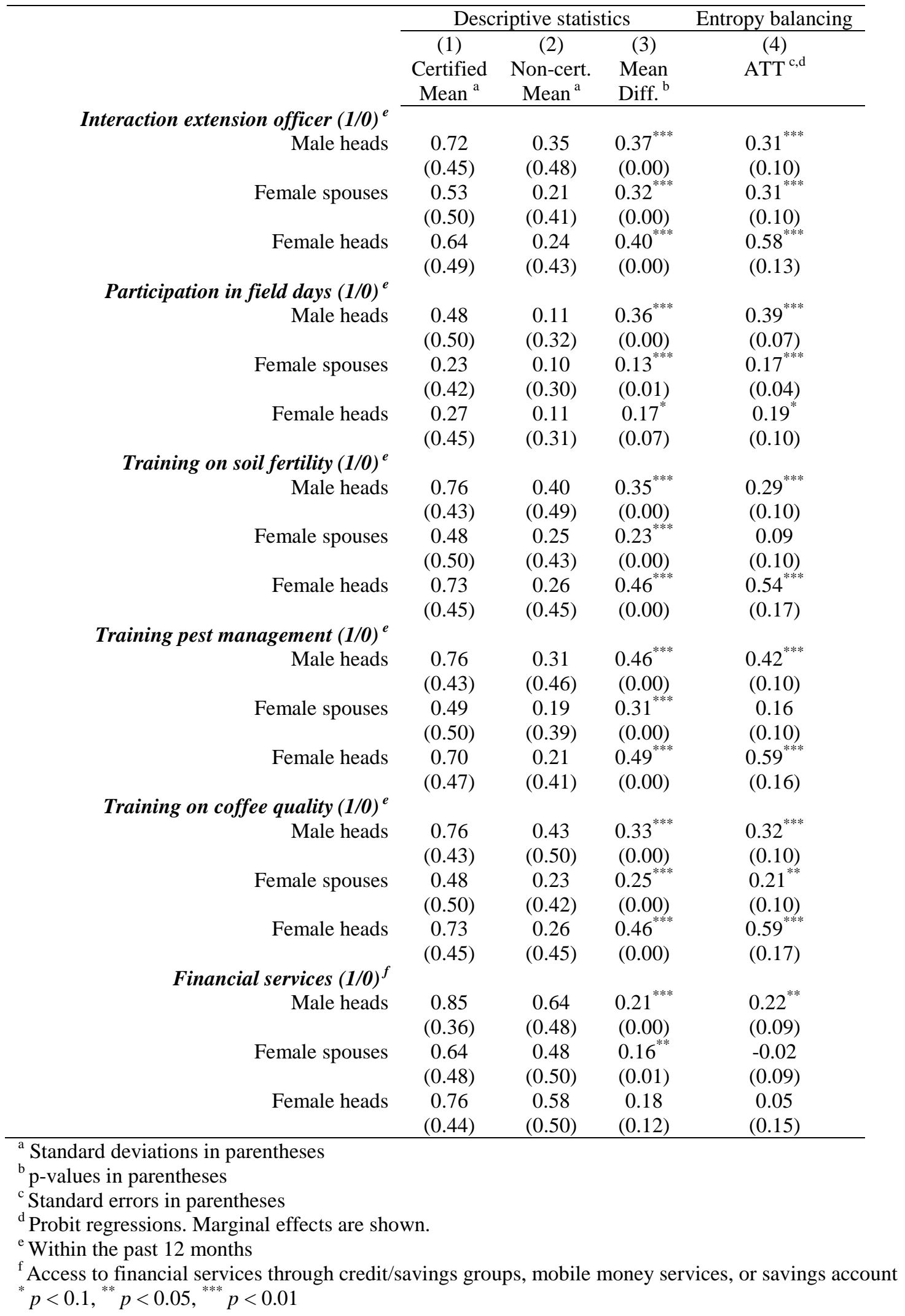




\subsubsection{Robustness checks}

As a robustness check, we estimate treatment effects for all outcomes using IPW. Results are displayed in the appendix (table A5-A7). Overall, these robustness checks support our findings, especially regarding the sign of the effects. However, in some cases, IPW estimates are significant at a higher level than estimates obtained through entropy balancing. Given these differences, we prefer using the more conservative estimates, obtained through entropy balancing. In many instances, the magnitude of the treatment effect is similar using entropy balancing and IPW. For instance, we find that standards increase the value of female spouses' assets by 146,780 UGX (using entropy balancing) or 147,650 UGX (using IPW) (see table 10 and table A5). However, in other cases, IPW and entropy balancing estimates are dissimilar, especially for groups with few observations (e.g. female-headed households). The exact magnitude of treatment effects should hence be interpreted with caution.

\subsection{Discussion and conclusion}

Achieving gender equality remains a challenge, especially in rural areas of developing countries, where poverty is particularly persistent. The literature suggests that agricultural commercialization may further increase gender disparities. Here, we have analyzed whether sustainability standards can contribute to mitigating such adverse effects on gender equality. We have focused on two sustainability standards that specifically address gender issues in cash crop production, namely Fairtrade and UTZ. Based on a household survey and gender-disaggregated data, we have analyzed the effect of sustainability standards on individuals in male-headed households (i.e. on male household heads and female spouses) and in female-headed households (i.e. on female household heads). Our results suggest that sustainability standards may not completely eliminate gender disparities, but can at least contribute towards this goal.

Our results regarding economic empowerment are promising and challenge earlier conclusions that economic benefits from sustainability standards are captured by men (Bolwig 2012; Sen 2014; Loconto 2015). Our results suggest that a differentiation between male-headed and female-headed households is useful, given heterogeneous effects. We hypothesized that sustainability standards may contribute to women's economic empowerment (1) by improving women's access to markets and income or (2) by increasing women's control over income from cash crop production. We find that the fist holds for female household heads; and the latter for female spouses. In female- 
headed households, sustainability standards increase total household assets and women's individual assets almost to the same extent. This is because most assets in female-headed households are owned by female heads. The positive welfare effect possibly stems from higher coffee revenues among certified households. Female household heads are usually responsible for coffee production and marketing, so that they directly benefit from the access to high value markets and higher incomes. More generally, these findings support that cash crops are seldom produced only by men and that the label 'men's crop' may not be applicable (Doss 2002; Orr et al. 2016).

In male-headed households, standards do not only increase household assets, they also affect the distribution of wealth within households. Specifically, standards increase the value of women's assets and jointly held assets significantly. Apart from this absolute increase, standards also increase the share of assets held jointly by male heads and female spouses. We also show that women in certified households have greater control over coffee and crop revenues than their non-certified counterparts. This may explain why women are able to increase their asset stock. Our results are promising because we find that women can benefit economically from standards - even if they are not directly involved in marketing. In fact, we find that standards to not challenge male heads' dominant role coffee production and marketing at all. Standard may affect the distribution of wealth within households through different channels. Our results suggest that standards increase overall household welfare (proxied here using the value of household assets). In wealthier households, wealth may be more equally distributed among household members. Further, standards may increase peoples' preferences for a more equal distribution of wealth - though workshops, policies, and rules on nondiscrimination and gender equality, implemented by certified farmer organizations.

Our results also differ from previous, qualitative studies regarding women's workload. We find no evidence that sustainability standards increase women's workload. Our results may differ from previous results (Lyon, Bezaury, and Mutersbaugh 2010; Bolwig 2012), because our sample farmers are not Organic certified - and can thus use chemical pesticides. However, our data do not capture seasonal differences and may thus disguise how the burden of work is distributed throughout the year. For instance, UTZ and Fairtrade include requirements on coffee quality. Meeting these requirements presupposes careful and repeated picking of coffee cherries. Although harvesting is usually a joint activity (i.e. undertaken by all household members) the burden of work during the harvest seasons may be unequally distributed within households. 
Results further indicate that sustainability standards improve farmers' access to agricultural information, irrespective of gender. However, male and female household heads benefit more from sustainability standards than female spouses in this regard. Female spouses are also less likely than their husbands to register as a farmer group member and to participate in farmer group meetings. As Lyon (2008) points out, being an active, registered member is a precondition to influence decisions on which services are provided by certified farmer organizations. We also find that sustainability standards improve men's access to financial services - but not women's access to financial services. Thus, more could be done to improve especially female spouses' access to agricultural services and to encourage their registration as farmer organization members.

One may argue that sustainability standards fail to challenge traditional gender roles and inequalities, if men's dominance in cash crop marketing and farmer organizations persists (Sen 2014; Loconto 2015). However, expectations regarding the effect of sustainability standards or other development interventions should be realistic. Gender equality should be understood as a long-term process. Standards alone will not eliminate gender disparities. Thus, although we find that standards cannot address gender disparities in all respects, we consider the positive trend promising. It should however be emphasized that gender components of sustainability standards are usually suggestions to certified farmer organizations rather than mandatory certification requirements. As a result, it depends on the farmer organization and contextual factors whether or not gender issues are accounted for in implementing standards. In the two farmer organizations in Uganda, gender policies were implemented. Elsewhere this may not always be the case. Thus, we agree with previous qualitative studies (Lyon 2008; Hutchens 2010) that gender issues could be addressed more explicitly in standards - for instance in the form of mandatory certification requirements. Thereby, sustainability standards may promote gender equality in certified households more broadly.

We want to point at a number of shortcomings of our study to encourage future research into this direction. Although our findings indicate that standards can contribute to improving gender equality, the exact magnitude of our estimates should be interpreted with caution. This holds especially for subgroups for which the sample size is small (i.e. female-headed households). Moreover, we are not able to capture possible spill-over effect. Certified farmers are strongly encouraged to participate in trainings on gender equality and farming management. However, non-certified members of the certified farmer organizations are not explicitly excluded from such trainings. They may also be influenced by gender-sensitive rules and policies. As we do not account for possible 
spill-over effects in our analysis, we may underestimate the effect of sustainability standards on gender equality. Future research into this direction would be useful to obtain more precise treatment effects.

Further, we have used observational, cross-sectional data for our impact assessment. As a result, we cannot rule out possible selection bias. We have used entropy balancing to control for observed heterogeneity; and estimates on farmers' willingness to accept (WTA) sustainability standards to reduce possible bias from unobserved heterogeneity. Future studies could employ research designs and methods that are better suited to deal with possible issues of selection bias.

To assess individual-level impacts, we have used data collected at the household level and at the individual level. We suggest there is a potential trade off in using either of these approaches, which has received little attention so far. Specifically, data obtained at the household level may suffer from bias, because households members may have different perceptions regarding their and other's decision making authority (Anderson, Reynolds, and Gugerty 2017) or asset ownership. In other words, interviewing only one household member (usually the household head) may not provide the full picture. In contrast, data obtained at the individual level may suffer from selection bias, because interviewing several household members in each household may not always be possible. For instance, in our case, we could not interview all spouses, as some persons were not available when we arrived to conduct the survey. Given these two potential sources of bias, it is not clear which approach is more accurate to assess individual-level impacts. Nevertheless, we emphasize that we have no reasons to believe that we only interviewed particularly empowered women, nor that we failed to interview particularly disempowered women. 


\subsection{Appendix}

Table A4: Treatment probabilities (individual level)

\begin{tabular}{|c|c|c|c|}
\hline & $\begin{array}{c}\text { (1) } \\
\text { Male heads }\end{array}$ & $\begin{array}{c}\text { (2) } \\
\text { Female spouses }\end{array}$ & $\begin{array}{c}\text { (3) } \\
\text { Female heads }\end{array}$ \\
\hline Household size & $\begin{array}{c}0.02 \\
(0.01)\end{array}$ & $\begin{array}{c}0.01 \\
(0.01)\end{array}$ & $\begin{array}{l}-0.03 \\
(0.03)\end{array}$ \\
\hline Muslim (1/0) & $\begin{array}{l}-0.20^{*} \\
(0.10)\end{array}$ & $\begin{array}{l}-0.18^{*} \\
(0.10)\end{array}$ & $\begin{array}{l}-0.64^{* *} \\
(0.30)\end{array}$ \\
\hline Age (yrs.) & $\begin{array}{l}0.02^{* * * *} \\
(0.00)\end{array}$ & $\begin{array}{l}0.02^{* * *} \\
(0.00)\end{array}$ & $\begin{array}{c}0.00 \\
(0.01)\end{array}$ \\
\hline Age gap (age head - age spouse) & $\begin{array}{l}-0.01^{* * *} \\
(0.00)\end{array}$ & $\begin{array}{l}0.01^{*} \\
(0.00)\end{array}$ & \\
\hline Schooling (yrs.) & $\begin{array}{l}0.05^{* * *} \\
(0.01)\end{array}$ & $\begin{array}{l}0.06^{* * * *} \\
(0.01)\end{array}$ & $\begin{array}{c}0.03 \\
(0.02)\end{array}$ \\
\hline Education gap (schooling head - spouse) & $\begin{array}{l}-0.03^{* *} \\
(0.01)\end{array}$ & $\begin{array}{c}0.02 \\
(0.01)\end{array}$ & \\
\hline Yrs. growing coffee & $\begin{array}{l}-0.00 \\
(0.00)\end{array}$ & $\begin{array}{c}0.00 \\
(0.00)\end{array}$ & $\begin{array}{c}0.00 \\
(0.01)\end{array}$ \\
\hline Altitude (m) & $\begin{array}{l}0.00^{* * *} \\
(0.00)\end{array}$ & $\begin{array}{l}0.00^{* * * *} \\
(0.00)\end{array}$ & $\begin{array}{l}0.00^{* * * *} \\
(0.00)\end{array}$ \\
\hline Distance to input market $(\mathrm{km})$ & $\begin{array}{l}-0.01 \\
(0.01)\end{array}$ & $\begin{array}{l}-0.02^{* *} \\
(0.01)\end{array}$ & $\begin{array}{c}0.01 \\
(0.01)\end{array}$ \\
\hline Distance to output market $(\mathrm{km})$ & $\begin{array}{l}-0.00 \\
(0.01)\end{array}$ & $\begin{array}{l}-0.01 \\
(0.01)\end{array}$ & $\begin{array}{l}-0.03 \\
(0.02)\end{array}$ \\
\hline Wealthy ancestors $(1 / 0)$ & $\begin{array}{l}-0.09 \\
(0.09)\end{array}$ & $\begin{array}{l}-0.16^{*} \\
(0.09)\end{array}$ & $\begin{array}{c}0.18 \\
(0.18)\end{array}$ \\
\hline WTA sustainability standards & $\begin{array}{c}0.00 \\
(0.01)\end{array}$ & $\begin{array}{c}0.00 \\
(0.01)\end{array}$ & $\begin{array}{l}0.05^{* *} \\
(0.02)\end{array}$ \\
\hline Observations & 233 & 244 & 71 \\
\hline $\begin{array}{l}\text { Pseudo } \mathrm{R}^{2} \\
\text { LR chi }\end{array}$ & $\begin{array}{c}0.19 \\
61.63^{\text {**** }}\end{array}$ & $\begin{array}{l}0.22 \\
74.84^{* * * *}\end{array}$ & $\begin{array}{c}0.25 \\
24.90^{* * * *}\end{array}$ \\
\hline
\end{tabular}

Probit estimates, marginal effects are shown

${ }^{*} p<0.1,{ }^{* *} p<0.05,{ }^{* * *} p<0.01$ 
Table A5: Asset ownership (in 1000 UGX)

\begin{tabular}{|c|c|c|}
\hline \multirow[b]{3}{*}{ Male-headed households } & \multicolumn{2}{|c|}{ IPW } \\
\hline & ATT & SE \\
\hline & & \\
\hline Value household assets & $1148.58^{* * * * *}$ & $(348.19)$ \\
\hline Asset ownership male head & & \\
\hline Value & 109.06 & $(254.95)$ \\
\hline Percent of total household assets & $-10.52^{* *}$ & $(4.80)$ \\
\hline Asset ownership female spouse & & \\
\hline Value & $147.65^{* *}$ & $(69.78)$ \\
\hline Percent of total household assets & -0.55 & $(2.41)$ \\
\hline Joint asset ownership & & \\
\hline Value & $926.86^{* * *}$ & $(220.69)$ \\
\hline Percent of total household assets & $12.43^{* * *}$ & $(4.65)$ \\
\hline Female-headed households & & \\
\hline Value household assets & $1775.60^{* * * *}$ & $(554.28)$ \\
\hline $\begin{array}{l}\text { Asset ownership female head } \\
\text { Value }\end{array}$ & $140925^{* * * *}$ & $(53030)$ \\
\hline Percent of total household assets & -7.13 & $(6.31)$ \\
\hline
\end{tabular}

Table A6: Workload and social capital

\begin{tabular}{|c|c|c|}
\hline \multirow{3}{*}{ Workload and satisfaction } & \multicolumn{2}{|c|}{ IPW } \\
\hline & ATT & SE \\
\hline & & \\
\hline \multicolumn{3}{|l|}{ Farm, off-farm, and domestic work (hrs./day) } \\
\hline Male heads & -0.07 & $(0.50)$ \\
\hline Female spouses & 0.17 & $(0.59)$ \\
\hline Female heads & 0.19 & $(0.68)$ \\
\hline \multicolumn{3}{|l|}{ Satisfaction leisure time $(1-5)^{e}$} \\
\hline Male heads & 0.00 & $(0.17)$ \\
\hline Female spouses & -0.01 & $(0.22)$ \\
\hline Female heads & -0.60 & $(0.40)$ \\
\hline \multicolumn{3}{|l|}{ Social capital } \\
\hline \multicolumn{3}{|l|}{ Participation in farmer meetings (1/0) } \\
\hline Male heads & -0.04 & $(0.05)$ \\
\hline Female spouses & $0.16^{*}$ & $(0.08)$ \\
\hline Female heads & 0.07 & $(0.16)$ \\
\hline \multicolumn{3}{|l|}{ Administrative / leadership position (1/0) } \\
\hline Male heads & $0.17^{* *}$ & $(0.08)$ \\
\hline Female spouses & $0.20^{* * *}$ & $(0.08)$ \\
\hline Female heads & -0.03 & $(0.16)$ \\
\hline
\end{tabular}


Table A7: Access to extension and financial services

\begin{tabular}{rcc}
\hline & \multicolumn{2}{c}{ IPW } \\
& ATT & SE \\
\hline Interaction extension officer (1/0) & & \\
Male heads & $0.35^{* * *}$ & $(0.08)$ \\
Female spouses & $0.34^{* * *}$ & $(0.07)$ \\
Female heads & $0.45^{* * *}$ & $(0.13)$
\end{tabular}

Participation in field days (1/0)

Male heads $0.39^{* * *}(0.05)$

Female spouses $0.15^{* * *} \quad(0.05)$

Female heads $\quad 0.09 \quad(0.12)$

Training on soil fertility (1/0)

Male heads $0.31^{* * * *}(0.08)$

Female spouses $0.19^{* * *} \quad(0.07)$

Female heads $0.43^{* * *} \quad(0.13)$

Training pest management (1/0)

Male heads $0.44^{* * *} \quad(0.07)$

Female spouses $0.26^{* * *} \quad(0.07)$

Female heads $\quad 0.50^{* * * *} \quad(0.13)$

Training on coffee quality (1/0)

Male heads $0.32^{* * *}(0.08)$

Female spouses $\quad 0.27^{* * * *} \quad(0.07)$

Female heads $0.46^{* * *}(0.13)$

Financial services (1/0)

Male heads $\quad 0.22^{* * * *} \quad(0.07)$

\begin{tabular}{lll} 
Female spouses $\quad 0.02 \quad(0.07)$ \\
\hline
\end{tabular}

\begin{tabular}{lll} 
Female heads $\quad 0.07 \quad(0.12)$ \\
\hline
\end{tabular}

${ }^{*} p<0.1,{ }^{* * *} p<0.05,{ }^{* * *} p<0.01$ 


\title{
4 Towards improving the design of sustainability standards - A gendered analysis of farmers' preferences $^{20}$
}

\begin{abstract}
Recently, sustainability standards have gained in importance for export crops produced in developing countries. Several studies analyzed whether such standards deliver on their promise to improve the livelihoods of poor farmers, with mixed results. Here, we ask whether the design of standards could be improved such that farm households benefit more. An assessment of what particular features of standards hamper or facilitate participation requires a better understanding of farmers' preferences. Our contribution is twofold: First, based on a choice experiment we analyze how farmers evaluate actual and hypothetical features of standards. Data were collected from smallscale coffee producers in Uganda. Second, this is the first quantitative study on standards employing a gendered research design. A gender focus is important, because coffee and other certified export crops are often controlled by men. The choice experiment included features of standards aimed at reducing gender inequality and was conducted separately with male and female members of farm households. Results indicate that farmers have positive attitudes towards sustainability standards in general. While they dislike bans of productivity-enhancing inputs, agricultural training and special female support are appreciated. Many also see requirements that have to be met for certification as a welcome nudge to invest in better farm management and quality upgrading. Female farmers have a higher preference for standards than male farmers. Also within households, significant preference heterogeneity between women and men is found.
\end{abstract}

Keywords: Choice experiment, farmer preferences, sustainability standards, gender, mixed logit models

\footnotetext{
${ }^{20}$ This paper is accepted for publication in Word Development. It is co-authored by Prakashan C. Veettil (PCV) and Matin Qaim (MQ). I (EM) developed the research idea, collected the survey data in 2015, did the data analysis, and wrote the paper. PCV and MQ commented at the various stages of the research and contributed to writing and revising the paper.
} 


\subsection{Introduction}

Recently, sustainability standards and certification schemes have gained in importance for export crops produced in developing countries. Particularly remarkable is the development of the certified coffee sector. The area under certified coffee tripled from about one million to about three million hectares between 2008 and 2013. In 2013, an estimated 30 percent of the global coffee area was certified under one of the main certification schemes - 4C Association, Fairtrade, Organic, Rainforest Alliance, and UTZ (ITC 2015). ${ }^{21}$ This rapid spread is attributable to different factors. Sustainability standards address environmental, human rights, and welfare issues along agricultural value chains. An increasing number of consumers is willing to pay for such processrelated attributes (ITC 2011; Potts et al. 2014). Further, development agencies have played a key role in promoting and facilitating famer participation in certification schemes (Bacon 2005; Handschuch, Wollni, and Villalobos 2013). Increasingly, private companies also evolve as important players. More and more public and private sector organizations develop new sustainability standards. According to the International Trade Center, there are now over 200 different standards with a focus on sustainability (ITC 2016).

The literature about various aspects of sustainability standards is growing. One strand of literature analyzes whether such standards actually deliver on their promise to promote environmental sustainability (Méndez, Bacon, Olson, Morris et al. 2010; Blackman and Naranjo 2012; Kleemann and Abdulai 2013) and socioeconomic development (Bacon 2005; Bolwig, Gibbon, and Jones 2009; Jones and Gibbon 2011; Chiputwa, Spielman, and Qaim 2015) in smallholder production of coffee and other tropical export crops. The results are mixed. A few studies suggest that the price premium farmers receive is sometimes too small to recover the costs associated with certification (Beuchelt and Zeller 2011; Weber 2011; Ibanez and Blackman 2016). Others find that farmers benefit from higher output prices and agricultural and social services often provided in certification schemes (Bolwig, Gibbon, and Jones 2009; Jones and Gibbon 2011; Kleemann, Abdulai, and Buss 2014). Concrete outcomes seem to depend on the type of standard and a variety of contextual factors. A second strand of literature explores

\footnotetext{
${ }^{21}$ The total area under coffee is about 10 million hectares (FAO 2015). The figures regarding the certified area are an approximation. A major challenge in this estimation is the lack of information on the area that is double certified (e.g. Organic + Fairtrade). See ITC (2015) for further information.
} 
determinants of farmers' adoption of sustainability standards, particularly exploring whether marginalized households are possibly excluded (Kersting and Wollni 2012; Handschuch, Wollni, and Villalobos 2013).

Here, we ask whether the design of sustainability standards could be improved such that farm households benefit more. We explicitly focus on smallholder farmers' subjective preferences, without addressing the broader question as to how standards should optimally look like in order to contribute to sustainability in its various dimensions. This broader question - while also highly relevant - is beyond the scope of this article. ${ }^{22}$ The smallholder perspective is partial but important, because many of the world's poor depend on the small farm sector as their main source of income and employment. The proliferation of sustainability standards in international food markets means that more and more smallholders are directly or indirectly affected.

A good understanding of the features of standards that are particularly liked and disliked by farmers is important to better tailor related programs to smallholder conditions. Yet, the available evidence in this direction is scant. Existing impact studies focus on the overall - effect of specific standards such as Fairtrade and Organic. Thus, very little is known on how specific design attributes (e.g. the price premium, pesticide bans, agricultural training, or rules on postharvest management) contribute to the overall effect. Similarly, existing studies on determinants of farmers' adoption of sustainability standards focus on the decision to adopt a specific standard. It remains unclear which of the design attributes are particularly difficult for farmers to comply with. Standards with varying design attributes are usually not observed in the same setting, so observational data alone are of limited value. We employ a choice experimental approach to analyze farmers' preferences for sustainability standards in general, and for specific design attributes in particular.

Focusing on farmers' preferences is important for at least two reasons. First, it is known that farmers' preferences can influence their decision on which marketing channel to use, whether or not to participate in voluntary contractual agreements (Schipmann and Qaim 2011; Ochieng, Veettil, and Qaim 2017), or to adopt new farming practices (Ward

\footnotetext{
${ }^{22}$ Determining to what extent sustainability standards contribute to sustainable development from a more holistic perspective would require a multi-method approach and data collection at various stages of national and international supply chains to capture the multiple dimensions of sustainability and the diverse views of the many actors involved. Such research needs to build on suitable combinations of quantitative tools with qualitative methods and participatory approaches (Janssen and Anderies 2013; Méndez et al., 2013).
} 
et al. 2014; Marenya, Smith, and Nkonya 2014). By better addressing farmers' needs and preferences, adoption of sustainability standards may become more attractive and feasible for a larger number of farmers. Second, addressing farmers' preferences can be promoted as a goal in itself. Over the last two decades, community-driven and participatory approaches to poverty reduction have become more popular in the research and development community (Méndez, Bacon, and Cohen 2013). Against this background, organizations that set and define sustainability standards have increasingly introduced participatory mechanisms to ensure that farmers' points of view are properly considered (Potts et al. 2014). For instance, Fairtrade International and the International Federation of Organic Agriculture Movements (IFOAM) emphasize their commitment to including the voices of their members in developing and modifying standards. ${ }^{23}$

In our choice experiment, we differentiate between the preferences of male and female farmers. It is well known that technical and institutional innovations can affect men and women differently (Alderman et al. 1995; Doss 2001). Sustainability standards often focus on tropical export crops, such as coffee or cocoa. While women tend to provide a substantial amount of labor for cash crop production, they are often less involved in the marketing of these crops than men, as evidence from Africa, Asia, and Latin America shows (Lyon 2008; Sen 2014; Chiputwa and Qaim 2016). ${ }^{24}$ A few studies therefore conclude that men capture most of the economic benefits from certification (Lyon 2008; Bolwig 2012; Sen 2014; Loconto 2015). Also, certain certification requirements - such as the ban of herbicides - may further increase the workload of women (Bolwig 2012). Finally, farmer organizations, which play an important role in certification, are often the domain of men. For instance, about 80 percent of the registered members of Fairtrade certified farmer organizations are men (Fairtrade International 2009). As a result, women may have little say in decisions on services to be provided and projects to be

\footnotetext{
${ }^{23}$ IFOAM is the umbrella organization for Organic agriculture. While various Organic standards exist, many are based on - or considered equivalent to - standards developed by IFOAM. IFOAM's standards were developed through a participatory process and were approved by its members. IFOAM's members and affiliates are based in over 100 countries and represent stakeholders along agricultural value chains. IFOAM also supports participatory certification (participatory guarantee systems). Participatory certification is an alternative to third party certification. For further information see IFOAM's homepage (ifoam.bio/en).

${ }^{24}$ There can be exceptions to this typical gender pattern in specific situations. For instance, Lyon, Bezaury, and Mutersbaugh (2010) found that in some regions of Central America, where many men migrated to urban areas, female farmers are strongly involved in marketing Organic coffee. Kloos \& Renaud (2014) found that women engagement in Organic cotton production was accepted in Benin, because Organic production does not require the handling of pesticides, which is considered a male task in the local context.
} 
implemented (Lyon 2008; Bacon 2010; Sen 2014). ${ }^{25}$ Related challenges and opportunities are increasingly recognized by standard-setting bodies, because gender equality is an important component of sustainable development (Fairtrade International 2011a). Scientific evidence suggests that gender policies introduced through sustainability standards can be effective in improving women's bargaining power (Chiputwa and Qaim 2016).

Our contribution to the literature is threefold. First, we propose a new way to think about sustainability standards, namely as a package of requirements (e.g. the use of pesticides is prohibited; quality requirements have to be met) and benefits (e.g. farmers are paid a price premium; they are offered training, credit, or inputs). These requirements and benefits could be combined in various ways, which is particularly relevant when designing new standards or trying to further improve existing ones.

Second, we use a choice experiment - designed building on participatory methods - to analyze farmers' preferences for specific certification requirements and benefits. The empirical analysis focuses on smallholder coffee producers in Uganda. We are aware of only three choice experimental studies related to sustainability standards (Hope, Borgoyary, and Agarwal 2008; Ibnu et al. 2015; Vlaeminck et al. 2015). These studies look at concrete cases of existing standards, such as Fairtrade and Organic. We add to this literature by examining farmers' preferences for more generic, hypothetical attributes of sustainability standards, involving economic, social, and environmental components.

Third, this is the first quantitative study on sustainability standards with a comprehensive gendered research design. In the choice experiment, we include attributes of standards that specifically focus on gender issues and support for female farmers. In addition, we build on gender-disaggregated data to capture the perspective of different individuals within each household. In each household, we interviewed a male and a female household member. We also account for gender in the econometric analysis. We compare preferences of men and women and further disaggregate the group of women into female spouses and female household heads.

\footnotetext{
${ }^{25}$ For instance, under Fairtrade only registered members can serve on the organizations' boards. Only board members have voting rights on how to spend the Fairtrade premium. The Fairtrade premium is money paid to farmer organizations that can be spent on projects to promote economic and social development.
} 


\subsection{Data and background}

\subsubsection{Coffee in Uganda}

Over 70 percent of Uganda's population live in rural areas and have agricultural landholdings (UBOS 2016). Coffee is the country's main foreign-exchange earner and a major source of income and employment for 3.5 million families (UCDA 2016). Over 60 percent of Uganda's coffee production is exported to the European Union (UIA 2016). Both Arabica and Robusta coffee are grown. Yet, the main share of the country's coffee production (85 precent) is Robusta, which is grown at altitudes up to 1200 meters. Uganda has two harvest seasons for coffee: March-June and SeptemberNovember (UIA 2016).

Robusta coffee is grown predominantly by smallholder farmers. The average farm size ranges from 0.5 to 2.5 hectares. Coffee is usually intercropped with bananas and beans. Shade trees are traditional elements in this farming system (UCDA 2016; UIA 2016). Most farmers use few inputs and rely on family labor. Access to agricultural services, such as trainings and credits, is limited (UBOS 2010). Other challenges facing the Ugandan coffee sector are coffee diseases, poor soil management and fertility, and use of old, unproductive coffee trees. Average yields are low $(750 \mathrm{~kg}$ per hectare for Robusta). Poor infrastructure and volatile world market prices for coffee further reduce farmers' profits (UCDA 2016).

With some regional variation, about 80 percent of the coffee-producing households in Uganda are headed by males (UBOS 2010). As in most other African countries, coffee is a male-dominated crop. Women tend to have little control over coffee revenues and production decisions, even though they provide a substantial share of the manual labor.

\subsubsection{Household survey}

To select households for the survey and choice experiment, we used a multi-stage sampling strategy. We purposively selected three farmer organizations in Luwero and Masaka districts, central Uganda, where Robusta coffee is grown. All three selected farmer organizations are certified. One farmer organization is certified under Fairtrade, one under UTZ, and one under Organic standards. However, for individual farmers, certification is a voluntary decision. Thus, all three farmer organizations have certified and non-certified members. Based on complete membership lists provided by each farmer organization, we stratified between certified and non-certified members before 
randomly selecting households to be included. In total, we included 453 households (see table 13).

Table 13: Number of sample households by certification status

\begin{tabular}{lcc}
\hline Certification status & $\begin{array}{c}\text { Number of } \\
\text { households }\end{array}$ & $\begin{array}{c}\text { Number of individuals } \\
\text { interviewed in these households }\end{array}$ \\
\hline Not certified & 193 & 302 \\
Fairtrade & 120 & 196 \\
Organic & 70 & 106 \\
UTZ & 70 & 110 \\
\hline Total & 453 & 714 \\
\hline
\end{tabular}

All selected households were visited in their homestead for conducting face-to-face interviews and the choice experiment. The interviews and the choice experiment were conducted by a team of local enumerators under the supervision of the researchers. To the extent possible, in each household we targeted primary and secondary decisionmakers, defined as household members above 18 years of age who make or influence decisions for the entire household. Decision-making relates to agricultural production and other types of household investments or purchases. We refer to the household head as the primary decision-maker. The secondary decision-maker is usually the spouse of the household head. In households where these structures did not apply (e.g. femaleheaded households), we assessed whether there are other adult decision-makers, whom we interviewed if they were of the opposite sex as the primary decision-maker.

In total, we interviewed 397 primary and 317 secondary decision-makers (table 14). Male primary decision-makers are mostly married, whereas female primary decisionmakers are usually widowed, divorced, or single. Secondary decision-makers are mainly female spouses of male household heads; in some cases secondary decision-makers are older children or other relatives. All respondents were interviewed separately. In the Ugandan context, conducting separate interviews with male and female household members was possible and accepted. 
Table 14: Number of individual respondents by gender and decision-making power

\begin{tabular}{lcc}
\hline Respondent category & $\begin{array}{c}\text { Number of individuals } \\
\text { interviewed }\end{array}$ & $\begin{array}{c}\text { Total number of individuals } \\
\text { existing in each category }\end{array}$ \\
\hline $\begin{array}{l}\text { Male primary decision-maker (household } \\
\text { heads) }\end{array}$ & 307 & 355 \\
$\begin{array}{l}\text { Male secondary decision-maker (e.g. adult } \\
\text { sons in female-headed households) }\end{array}$ & 19 & 27 \\
$\begin{array}{l}\text { Female primary decision-maker (household } \\
\text { heads) }\end{array}$ & 91 & 99 \\
$\begin{array}{l}\text { Female secondary decision-maker (e.g. } \\
\text { spouses) }\end{array}$ & 297 & 331 \\
\hline Total & 714 & 812 \\
\hline
\end{tabular}

In some households, we were unable to conduct the interview and choice experiment with two individuals, either because a secondary decision-maker did not exist or because one of the individuals was absent. Table 14 shows that we were able to interview 88 percent of all existing primary and secondary decision-makers. Using data from the sociodemographic part of the household questionnaire, which captured key variables for all household members, we compared age and education between actually interviewed and absent individuals. For male primary and secondary decision-makers, as well as for female primary decision-makers, no statistically significant differences were detected. Only for female secondary decision-makers, we found slightly higher education levels $(\mathrm{p}=0.08)$ for absent than for actually interviewed individuals. Hence, we cannot rule out a certain level of self-selection bias in our sample. However, the comparisons suggest that the bias is small. Further, in our analysis we explicitly account for preference heterogeneity, so that possible underrepresentation of particular groups is not a major concern.

\subsection{Methods}

\subsubsection{Choice experiment}

We employ a choice experiment to analyze farmers' preferences for different features of sustainability standards. Choice experiments belong to the family of attribute-based methods, used to elicit information on preferences for actual or hypothetical products, services, or policies (Holmes and Adamowicz 2003). The method draws on Lancaster's theory of consumer choice and McFadden's extension of discrete choice theory 
(McFadden 1973). It is assumed that individuals derive utility from the attributes of a good, rather than from the good itself. Transferred to our case, we consider sustainability standards as a combination of design attributes. These attributes can be grouped into benefits (e.g. a price premium) and requirements (e.g. chemical pesticides are banned). When deciding whether or not to adopt a particular standard, farmers will evaluate the expected benefits and requirements. We are interested in possible genderspecific differences, so we analyze individual preferences of male and female household members.

Following the random utility framework, we can express utility associated with the adoption of a given standard as follows:

$\boldsymbol{U}_{i j}=\boldsymbol{V}_{i j}+\boldsymbol{e}_{\boldsymbol{i j}}=\alpha \boldsymbol{X}_{j}+\boldsymbol{\beta} \boldsymbol{Z}_{\boldsymbol{i}}+\boldsymbol{e}_{\boldsymbol{i j}}$ where $\quad \boldsymbol{U}_{\boldsymbol{i j}}=\left\{\begin{array}{c}\boldsymbol{U}_{\boldsymbol{M}_{i j}} ; \text { Utility for men } \\ \boldsymbol{U}_{\boldsymbol{F}_{i j}} ; \text { Utility for women }\end{array}\right.$

Utility $(U)$ for male $\left(U_{M}\right)$ or female $\left(U_{F}\right)$ farmer $i$ associated with standard $j$ can be decomposed into a deterministic element $(V)$ and a stochastic element $(e)$, where the latter captures unobserved factors that determine farmers' choices. The deterministic part can be further decomposed into a choice-specific part $(X)$ and an individual-specific part $(Z) . X$ is the vector of attributes of standard $j$ for which we want to measure farmers' preferences. $Z$ is a vector of individual, farm, household, and contextual characteristics that determine preferences and thus preference heterogeneity.

\subsubsection{Selection of standard attributes and attribute levels}

Sustainability standards usually encompass a set of detailed requirements concerning farming practices, post-harvest management, and, in some cases, community development projects. Our objective was to select attributes of standards that are relevant in the local context but also beyond. We used a multi-method approach (Janssen and Anderies 2013). Prior to implementing the structured survey of farmers, we conducted focus group discussions with six female groups, six male groups, and three mixed groups. Each group was composed of six to twelve farmers. During each session, we asked farmers to discuss and list all benefits and requirements they associate with sustainability standards. All points mentioned were listed on a large sheet of paper. Based on these lists, we asked farmers to rank benefits and requirements by subjectively-felt level of importance. In addition, we conducted semi-structured key informant interviews with managers of the farmer organizations, coffee traders, and agricultural extension officers. Results from these focus group discussions and key 
informant interviews helped us in selecting relevant attributes for use in the choice experiment.

We selected six attributes with two to six levels (table 15). The last level for each attribute is referred to as the base scenario, characterizing the situation of non-certified farmers. We classify attributes as benefits or requirements, as explained in the following.

Table 15: Overview of attributes and attribute levels used in the choice experiment

\begin{tabular}{|c|c|}
\hline Attributes & Attribute levels \\
\hline \multicolumn{2}{|l|}{ Benefits } \\
\hline \multirow[t]{6}{*}{ Price/price premium } & 1. Market price $+500 \mathrm{UGX} / \mathrm{kg}$ \\
\hline & 2. Market price $+400 \mathrm{UGX} / \mathrm{kg}$ \\
\hline & 3. Market price $+300 \mathrm{UGX} / \mathrm{kg}$ \\
\hline & 4. Market price $+200 \mathrm{UGX} / \mathrm{kg}$ \\
\hline & 5. Market price $+100 \mathrm{UGX} / \mathrm{kg}$ \\
\hline & 6. Market price \\
\hline \multicolumn{2}{|l|}{ Agricultural training } \\
\hline & 1. Agricultural training \\
\hline & 2. No agricultural training offered \\
\hline \multicolumn{2}{|l|}{ Gender policies } \\
\hline & 1. Training on gender equality \\
\hline & 2. Credits for women \\
\hline & 3. No training on gender equality and/or no credits for women offered \\
\hline \multicolumn{2}{|l|}{ Requirements } \\
\hline \multirow[t]{3}{*}{ Quality requirements } & 1. Only ripe cherries \\
\hline & 2. No drying on soil \\
\hline & 3. Picking unripe cherries optional and/or drying on soil optional \\
\hline \multicolumn{2}{|l|}{ Handling of pesticides } \\
\hline & 1. Pesticides prohibited \\
\hline & 2. Protective clothing mandatory \\
\hline & 3. Pesticides allowed and/or protective clothing optional \\
\hline \multicolumn{2}{|l|}{ Record keeping } \\
\hline & 1. Record keeping mandatory \\
\hline & 2. Record keeping optional \\
\hline
\end{tabular}

The first attribute is the price received for the coffee sold. The most apparent benefit of certification is a price premium. The price attribute has six levels, each one representing a different price premium per kilogram of red, sundried coffee cherries, locally referred 
to as kiboko. ${ }^{26}$ In the base scenario, farmers would receive the usual market price. At the time of the survey, the average price for kiboko was about 2000 Ugandan shillings (UGX) per kilogram (ca. 0.58 US dollar). To determine a range of realistic price premiums, we used information provided by farmer organizations, traders, and homepages of certification bodies.

The second attribute relates to agricultural training. Most certification agencies require certified farmer organizations to offer training on different topics, such as plant nutrition, pest control, or soil management. We expect that farmers have positive preferences for agricultural training as this may help to increase yields.

As the third attribute we consider two gender policies: credits for women ${ }^{27}$ and specific training on gender equality. The goal of gender training is to raise awareness on gender issues and help participants appreciate the work undertaken by other household members and to work as a team. Such awareness elements are trained with participatory methods (e.g. drawing maps to visualize the distribution of tasks). We learned from focus group discussions that such training elements were considered useful by farmers. For instance, one married couple explained how the existing gender training helped them realize that they are better-off if they share certain responsibilities in coffee production. For instance, while previously the female spouse did not feel responsible for coffee placed outside the house for drying, she would now make sure it is not exposed to rain when her husband is not around. Most certification bodies do not require farmer organizations to introduce such training, although training on gender roles is sometimes encouraged. Concerning the other gender policy considered in our choice experiment, we are not aware of any sustainability standard that recommends introducing credit schemes targeted specifically at women.

The fourth attribute concerns coffee quality, where we focus on harvesting and postharvest management. Practices such as picking unripe coffee cherries and drying cherries on the bare soil are common practice but can reduce coffee quality. Avoiding such practices can be time-intensive and costly for farmers. Picking only ripe cherries

\footnotetext{
${ }^{26}$ Farmers in Uganda sell their coffee as red cherries, kiboko (sundried cherries), or FAQ (dried and shelled coffee beans). Since most farmers sell their coffee as kiboko, we use the kiboko price as the reference point, even though we explained to participants that selling coffee in other forms is also possible with certification.

${ }^{27}$ In the descriptions, credit conditions were specified such that female farmers can obtain credits at an interest rate of three percent. The money is handed out to women only and has to be paid back within a period of three months.
} 
requires repeated picking in the same plot since not all cherries are ripe simultaneously. And drying coffee on tarpaulins or cemented floorboards is more expensive than drying on bare soil. Thus, we hypothesize that farmers dislike these quality requirements.

As the fifths attribute we include requirements on the handling of chemical pesticides. Most standards do not prohibit pesticides in general but restrict the use of chemicals that are known to be particularly harmful for the environment and/or human health. Because of health concerns, many standards also require the use of protective clothing during pesticide application. We expect that farmers dislike such restrictions since costs and labor requirements may increase, whereas yields may possibly decrease when chemical pesticides are banned.

The last attribute in the choice experiment is record keeping. Documentation is a precondition to ensure traceability - and thus to maintain consumers' trust in the sustainability label. While most of the documentation for certification is undertaken at the level of farmer organizations, some standards also require individual farmers to keep records about the type and quantity of inputs used, the timing of applications, and the costs and revenues of farm production. Record keeping is a challenge for illiterate farmers. Even literate farmers are often not used to formal paperwork. Hence, we expect farmers to dislike record-keeping requirements.

\subsubsection{Experimental design}

The six attributes and their different levels imply a full factorial design with $648\left(6 \times 3^{3}\right.$ $x 2^{2}$ ) combinations, each representing a theoretically possible standard. This large number is impracticable to use in a choice experiment. There are different approaches to reduce the number of alternatives, including orthogonal fractional and d-optimal designs. The decision between these approaches represents a trade-off between statistical efficiency (d-optimal design) and non-correlation between attributes (orthogonal design) (Hensher, Rose, and Greene 2005). We consider statistical efficiency more important. Hence we used the d-optimal design, accounting for the possibility that attributes might be correlated. Additionally, we excluded combinations that only had base scenario levels for either benefits or requirements, as these combinations would represent dominant and not very realistic alternatives. For instance, a price premium without any strings attached would be an unlikely offer in reality.

The remaining alternatives were randomly assigned to five blocks, each block containing six choice sets. Respondents were randomly assigned one block, so each 
farmer was asked to make six consecutive decisions. Each choice set had three alternatives to choose from. On the choice cards that we used (figure 5) the first two options with variations of the attribute levels were labelled 'certification scheme'. The third option on every choice card was the 'non-standard' alternative with base scenario levels for all attributes.

Figure 5: Example of a choice card

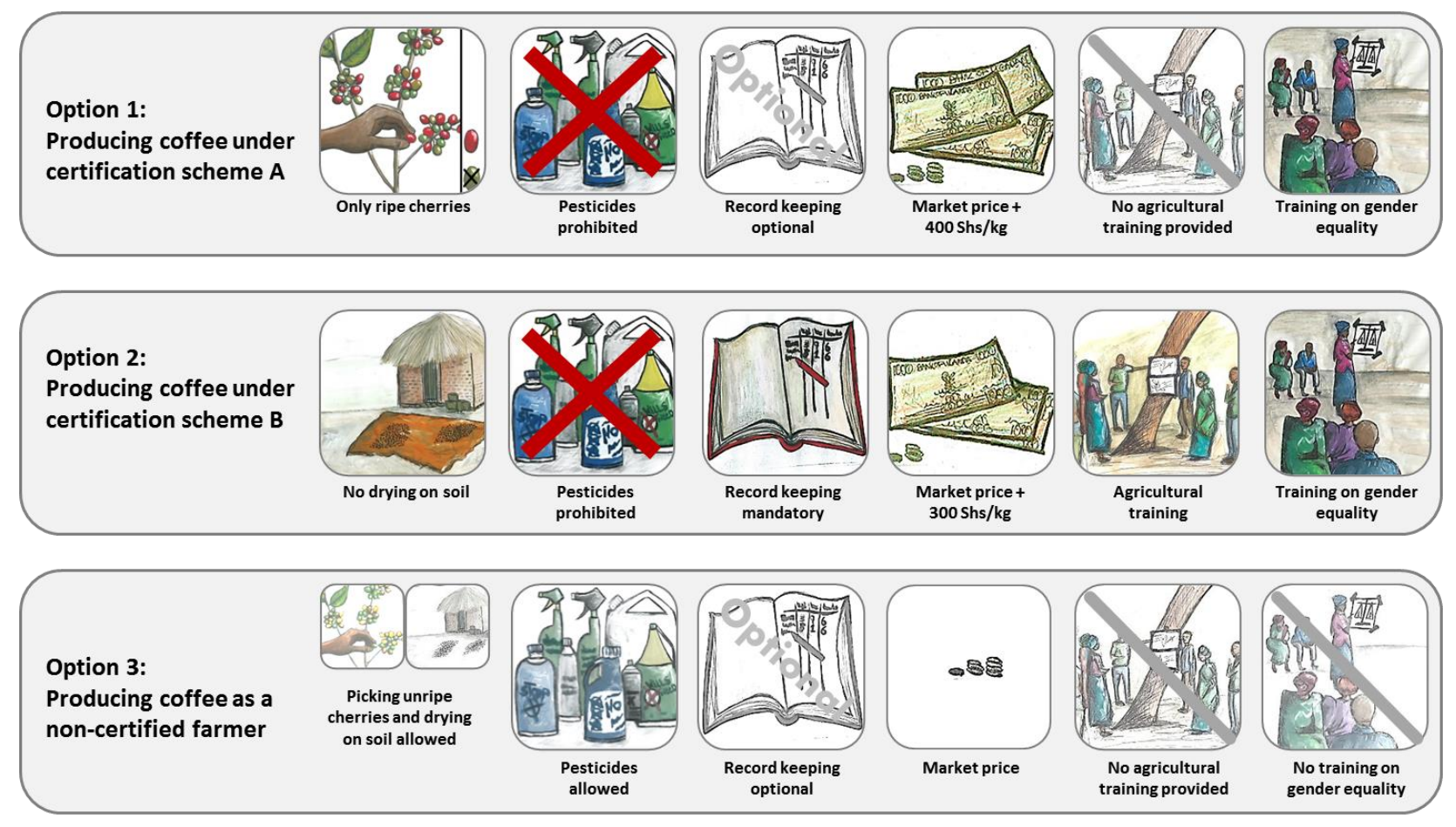

Source: Authors

\subsubsection{Econometric approach}

For the econometric analysis, we use mixed logit models (random parameter logit) that we estimate using maximum simulated likelihood (Train 2009). The mixed logit is frequently used in choice modelling as it relaxes some of the potentially unrealistic assumptions of the standard logit. Specifically, mixed logit models allow for preference heterogeneity and correlation of unobserved factors over time. Mixed logit models also relax the independence from irrelevant alternatives property (Train 2009).

Our models include an alternative specific constant (ASC) to account for the fact that the choice sets include a base scenario. The ASC is a dummy variable, coded 0 for the base scenario and 1 for the certification alternatives. By using effect-coding instead of dummy-coding we avoid correlation of the attribute estimates with the ASC (Bech and 
Gyrd-Hansen 2005). Thus, the ASC reflects general attitudes towards standards, capturing factors not included as attributes in the choice experiment. Such omitted variables may influence decision-making, as sample farmers are familiar with actual standards and may possibly think about attributes beyond those explicitly included.

After testing for correlated structures using the likelihood ratio test, we run all models with the specification that the random coefficients are correlated. Since we assume that farmers have a homogenous preference for higher coffee prices, we specify the price attribute to have a fixed coefficient. All other attributes and the ASC are specified as random and normally distributed, assuming that preference heterogeneity exists.

We run different model specifications. The base specification includes only the ASC and the attribute level as explanatory variables:

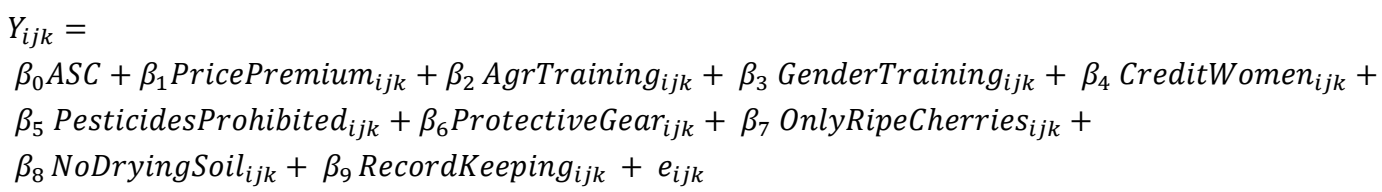

where $Y$ denotes the binary decision made by the male or female farmer $i$ for alternative $j$ and choice set $k$. This base specification allows us to assess if a given attribute level increases or decreases farmers' willingness to adopt the standard, as indicated by the sign of the coefficient.

In other model specifications, we additionally include interaction terms between the ASC or specific attribute levels and household or individual characteristics to explore some of the factors that drive preference heterogeneity. ${ }^{28} \mathrm{We}$ are particularly interested in gender effects across and within households, the latter of which we explore by differentiating between primary and secondary decision-makers. Furthermore, we expect that farmers' experience with existing standards may also influence their attitudes towards particular standard attributes.

\footnotetext{
${ }^{28}$ In order to analyze factors that contribute to preference heterogeneity in more detail, latent class models could also be used. We tried latent class models, but the additional insights were limited. Rather than fully exploring all drivers of preference heterogeneity, we are primarily interested in understanding how gender and experience with standards affect farmers' preferences. This can best be tested through including suitable interaction terms. Unexplained heterogeneity will not bias the estimation results in the mixed logit modeling framework.
} 


\subsection{Descriptive Statistics}

\subsubsection{Gender-specific differences}

Table 16 displays descriptive statistics. Column (1) shows the full sample of 714 individuals, whereas the other columns differentiate by gender. We compare men to female primary decision-makers and female secondary decision-makers.

Table 16: Summary statistics by gender (individual level)

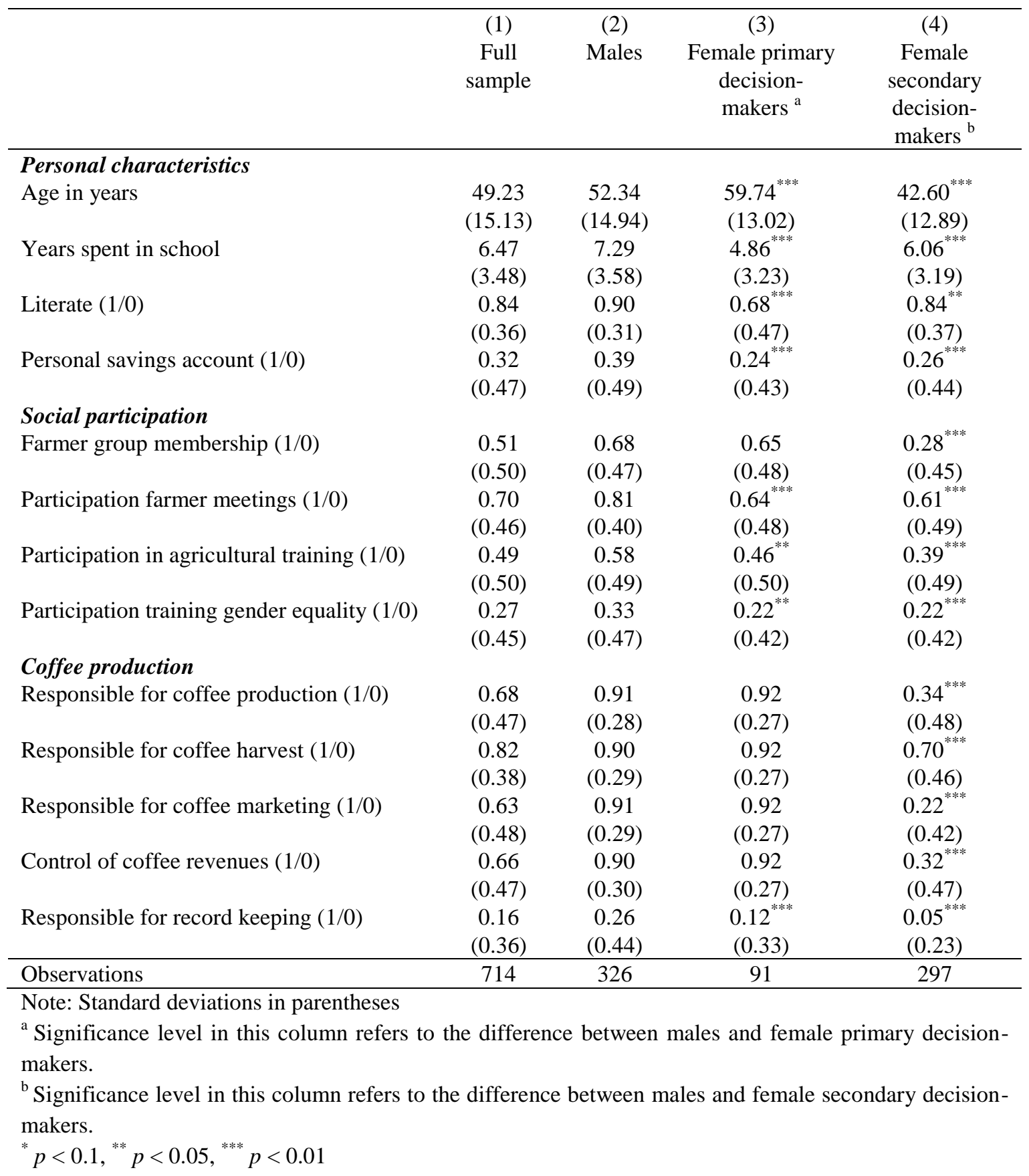


On average, female primary decision-makers are older than $\operatorname{men}^{29}$, whereas female secondary decision-makers are younger. Both types of female decision-makers are less educated and less likely to have a personal savings account than men. Females are also less likely to participate in training sessions and farmer group meetings. However, female primary decision-makers are as likely as men to be member of a farmer group.

Table 16 also provides an overview of gender-specific responsibilities in coffee production. In male-headed households, men usually hold the main responsibility in terms of production, harvesting, and sales. In most cases, they also control the revenues. However, in some of the households these responsibilities are shared with other household members, including female secondary decision-makers. Harvesting in particular often requires labor input from all household members. In female-headed households, the situation is different; female primary decision-makers usually take on responsibilities that are traditionally held by males.

\subsubsection{Differences between certified and non-certified households}

Table 17 also shows descriptive statistics, but now referring to the household level. In addition to the full sample of 453 households shown in column (1), we differentiate between non-certified households (column 2) and those that are certified under Fairtrade, Organic, and UTZ (columns 3-5). On average, the heads of certified households are better educated, except for Organic certified households. Fairtrade households have higher living standards (in terms of per capita expenditures and household assets), whereas UTZ households tend to have higher off-farm incomes.

There are also significant differences in terms of farm characteristics and coffee production practices: certified households have larger landholdings and larger areas grown with coffee. Fairtrade households are more likely to use pesticides than the other subsamples, whereas for Organic households the opposite holds true. In comparison to non-certified households, certified households are more likely to keep records and less likely to dry coffee on bare soil. Organic households are less likely to pick unripe cherries. These numbers are based on farmers' own reporting. As many of the listed practices are either recommended or discouraged by farmer organizations, the possibility of social desirability bias in these responses cannot be ruled out.

\footnotetext{
${ }^{29}$ Given the small number of male secondary decision-makers ( $N=19$ ), we do not disaggregate the group of men.
} 
Table 17: Summary statistics by standard (household level)

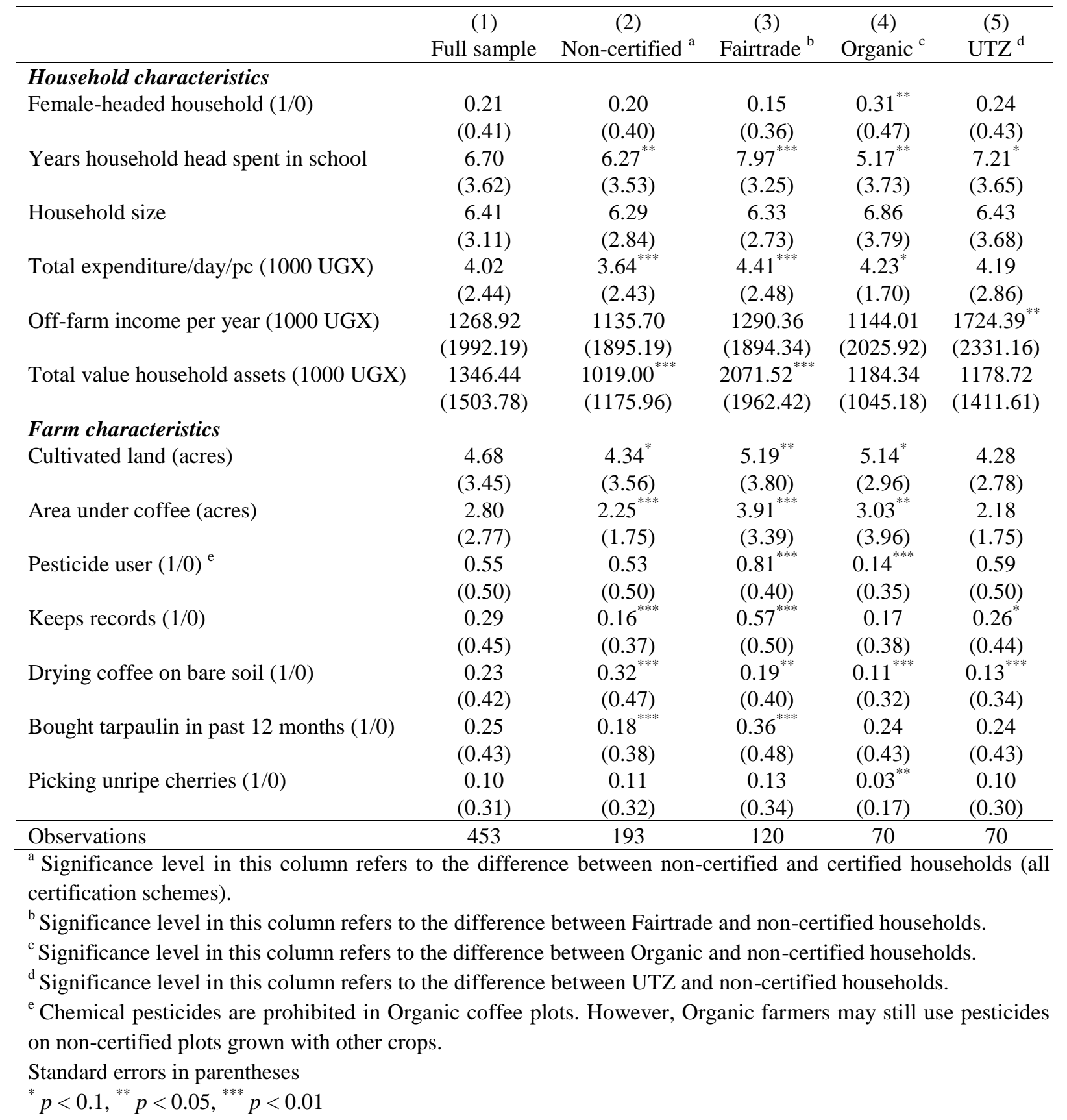




\subsection{Estimation results}

Estimation results for the first set of mixed logit models are shown in table 18 . Regardless of the exact specification, the ASC has a positive and significant coefficient, indicating that farmers have positive attitudes towards sustainability standards in general. Furthermore, the significant mean parameters for all attributes suggest that these attributes are relevant to farmers and affect their utility. Finally, the standard deviation parameters, which are shown in the lower part of table 18, confirm that significant preference heterogeneity exists. In the following, we discuss the model results in more detail, starting with preferences for the average farmer, before taking a closer look at factors influencing preference heterogeneity.

\subsubsection{General preferences for sustainability standards}

The base specification of the mixed logit model - with only the attributes included - is shown in column (1) of table 18. The coefficients of all attributes referred to as benefits (i.e. price premium, agricultural training, training on gender equality, and credits for women) have the expected positive sign. Farmers prefer sustainability standards that offer higher coffee prices, agricultural training, and gender policies. The latter result is particularly noteworthy; it suggests that promoting gender equality through sustainability standards is possible and appreciated.

Turning to the attributes referred to as requirements (i.e. handling of pesticides, quality requirements, and record keeping), only the coefficient of 'pesticides prohibited' has the expected negative sign. About half of the households in our sample use chemical pesticides, primarily herbicides. If pesticides are prohibited, more time would have to be spent on weeding, or yields would decrease. A negative preference for pesticide bans is therefore plausible. More surprising is that the other attributes on requirements have significantly positive coefficients. As discussed, compliance with these requirements can be associated with costs. Buying tarpaulins for drying coffee cherries or protective clothing for pesticide applications requires capital. Picking only ripe cherries requires additional labor; and record keeping requires some degree of discipline, or, for illiterate farmers, finding someone else to assist. 
Table 18: Mixed logit model estimates - Base specification and differences by certification status

\begin{tabular}{|c|c|c|c|c|c|}
\hline & (1) & (2) & (3) & (4) & (5) \\
\hline \multicolumn{6}{|l|}{ Mean parameters } \\
\hline ASC (certification) & $4.14^{* * * *}(0.83)$ & $1.63^{* * *}(0.42)$ & $1.87^{* * * *}(0.66)$ & $1.83^{* * * *}(0.45)$ & $2.04^{* * * *}(0.42)$ \\
\hline Price premium (in 100 UGX) & $0.34^{* * *}(0.05)$ & $0.33^{* * *}(0.04)$ & $0.33^{* * *}(0.04)$ & $0.34^{* * * *}(0.04)$ & $0.35^{* * * *}(0.04)$ \\
\hline Agricultural training $(1 / 0)^{\mathrm{a}}$ & $0.87^{* * *}(0.08)$ & $0.85^{* * *}(0.07)$ & $0.87^{* * *}(0.07)$ & $0.87^{* * *}(0.07)$ & $0.77^{* * *}(0.09)$ \\
\hline Training on gender equality $(1 / 0)^{b}$ & $0.68^{* * *}(0.12)$ & $0.68^{* * *}(0.11)$ & $0.66^{* * *}(0.11)$ & $0.72^{* * *}(0.10)$ & $0.65^{* * * *}(0.12)$ \\
\hline Credits for women $(1 / 0)^{b}$ & $0.54^{* * *}(0.09)$ & $0.50^{* * *}(0.08)$ & $0.50^{* * *}(0.08)$ & $0.53^{* * *}(0.08)$ & $0.63^{* * *}(0.09)$ \\
\hline Pesticides prohibited $(1 / 0)^{\mathrm{c}}$ & $-0.54^{* * * *}(0.11)$ & $-0.52^{* * *}(0.11)$ & $-0.52^{* * * *}(0.11)$ & $-0.53^{* * *}(0.12)$ & $-0.04(0.14)$ \\
\hline Protective clothing mandatory $(1 / 0)^{\mathrm{c}}$ & $0.65^{* * *}(0.10)$ & $0.67^{* * *}(0.09)$ & $0.66^{* * *}(0.09)$ & $0.74^{* * *}(0.10)$ & $0.65^{* * * *}(0.10)$ \\
\hline Only ripe cherries $(1 / 0)^{\mathrm{d}}$ & $1.28^{* * *}(0.14)$ & $1.38^{* * *}(0.13)$ & $1.42^{* * *}(0.13)$ & $1.44^{* * *}(0.13)$ & $1.49^{* * *}(0.14)$ \\
\hline No drying on soil $(1 / 0)^{d}$ & $0.28^{* * *}(0.10)$ & $0.31^{* * * *}(0.10)$ & $0.30^{* * *}(0.10)$ & $0.36^{* * *}(0.11)$ & $0.27^{* * *}(0.10)$ \\
\hline Record keeping $(1 / 0)^{\mathrm{e}}$ & $0.40^{* * *}(0.07)$ & $0.42^{* * *}(0.07)$ & $0.42^{* * *}(0.07)$ & $0.25^{* * *}(0.08)$ & $0.40^{* * * *}(0.07)$ \\
\hline \multicolumn{6}{|l|}{ ASC interactions $(1 / 0)$} \\
\hline ASC x Certified & & $0.38(0.26)$ & & & \\
\hline ASC x Fairtrade & & & $0.00(0.53)$ & & \\
\hline ASC x Organic & & & $1.10(0.87)$ & & \\
\hline ASC x UTZ & & & $1.34(0.83)$ & & \\
\hline \multicolumn{6}{|l|}{ Attribute interactions $(1 / 0)$} \\
\hline Pesticides prohibited $\mathrm{x}$ Fairtrade & & & & $-0.37^{* *}(0.17)$ & \\
\hline Pesticides prohibited $\mathrm{x}$ Organic & & & & $0.50^{* * *}(0.19)$ & \\
\hline Record keeping x Fairtrade & & & & $0.39^{* * *}(0.13)$ & \\
\hline Record keeping x UTZ & & & & $0.39^{* * *}(0.15)$ & \\
\hline Ag. training $\mathrm{x}$ participation ag. training & & & & & $0.31^{* * * *}(0.11)$ \\
\hline Training gender $\mathrm{x}$ particip. gender training & & & & & $0.39^{* *}(0.19)$ \\
\hline Pesticides prohibited $\mathrm{x}$ pesticide user & & & & & $-0.85^{* * *}(0.15)$ \\
\hline Record keeping x keeps records & & & & & $0.39^{* *}(0.15)$ \\
\hline \multicolumn{6}{|l|}{ Standard deviation parameters } \\
\hline ASC & $2.75^{* * * *}(0.71)$ & $0.82(0.65)$ & $0.13(0.46)$ & $1.74^{* * * *}(0.46)$ & $1.89^{* * * *}(0.50)$ \\
\hline Agricultural training & $0.46^{* * *}(0.13)$ & $0.47^{* * *}(0.08)$ & $0.48^{* * *}(0.08)$ & $0.50^{* * *}(0.09)$ & $0.57^{* * * *}(0.10)$ \\
\hline Training on gender equality & $0.53^{* * *}(0.14)$ & $0.61^{* * *}(0.16)$ & $0.58^{* * *}(0.14)$ & $0.55^{* * *}(0.16)$ & $0.61^{* * * *}(0.17)$ \\
\hline Credits for women & $0.81^{* * *}(0.14)$ & $0.89^{* * * *}(0.14)$ & $0.87^{* * * *}(0.14)$ & $0.66^{* * *}(0.12)$ & $0.99^{* * * *}(0.14)$ \\
\hline Pesticides prohibited & $1.55^{* * *}(0.16)$ & $1.62^{* * *}(0.15)$ & $1.64^{* * *}(0.14)$ & $1.52^{* * *}(0.13)$ & $1.58^{* * * *}(0.16)$ \\
\hline Protective clothing mandatory & $1.06^{* * *}(0.17)$ & $1.02^{* * *}(0.16)$ & $1.05^{* * *}(0.16)$ & $1.01^{* * *}(0.15)$ & $1.11^{* * *}(0.18)$ \\
\hline Only ripe cherries & $0.99^{* * *}(0.25)$ & $1.00^{* * *}(0.14)$ & $1.04^{* * *}(0.13)$ & $1.09^{* * *}(0.14)$ & $1.15^{* * *}(0.16)$ \\
\hline No drying on soil & $0.90^{* * *}(0.17)$ & $0.83^{* * *}(0.15)$ & $0.92^{* * *}(0.14)$ & $0.95^{* * *}(0.14)$ & $0.91^{* * * *}(0.17)$ \\
\hline Record keeping & $0.73^{* * * *}(0.16)$ & $0.71^{* * *}(0.12)$ & $0.72^{* * *}(0.11)$ & $0.78^{* * * *}(0.11)$ & $0.81^{* * * *}(0.12)$ \\
\hline Log Likelihood & -2375.33 & -2381.85 & -2381.24 & -2365.85 & -2352.05 \\
\hline Chi squared & $596.76^{* * *}$ & $572.75^{* * * *}$ & $571.85^{\text {**** }}$ & $568.48^{* * *}$ & $548.74^{* * *}$ \\
\hline Observations $(6 * 3 *$ no. of respondents $)$ & 12852 & 12852 & 12852 & 12852 & 12852 \\
\hline \multicolumn{6}{|l|}{ Note: Standard errors in parentheses } \\
\hline \multicolumn{6}{|l|}{${ }^{\mathrm{a}}$ Reference category is no agricultural training. } \\
\hline \multicolumn{6}{|c|}{${ }^{\mathrm{b}}$ Reference category is no gender policies (i.e. no training on gender equality and no credits for women). } \\
\hline \multicolumn{6}{|c|}{${ }^{\mathrm{c}}$ Reference category is no restrictions on pesticide use (i.e. pesticides allowed and protective clothing optional). } \\
\hline \multicolumn{6}{|c|}{$\begin{array}{l}\mathrm{d} \text { Reference category is no quality requirements (i.e. picking only ripe cherries and drying on tarpaulins both optional). } \\
{ }^{\mathrm{e}} \text { Reference category is record keeping optional. } \\
{ }^{*} p<0.1,{ }^{* *} p<0.05,{ }^{* * *} p<0.01\end{array}$} \\
\hline
\end{tabular}


However, these requirements are also associated with potential benefits that may accrue irrespective of an immediate price premium through the standard. More precisely, record keeping allows farmers to keep track of inputs and outputs and thus helps managing farm resources more efficiently. Similarly, compliance with safety and quality requirements can pay off: protective clothing for pesticide sprays can reduce possible health hazards; practices to improve coffee quality may help to fetch higher prices and reduce rejection rates by buyers. Hence, positive preferences for these attributes are not implausible. The question arising is why not more farmers use these practices, even without being a certification requirement, when the benefits are appreciated. For instance, only about 30 percent of the farm households keep records.

One reason might be that - in spite of a general willingness - compliance is simply not feasible for every farmer due to human capital constraints. Other possible reasons are self-control problems and present-biasedness, especially because the expected benefits do not occur immediately. In that case, certification may be seen as a welcome nudge to make investments, keep records, or allocate more labor to harvesting and post-harvest handling. It is well known that people often accept third-party monitoring to incentivize beneficial behavior. For instance, people attempt to overcome self-control problems through deadlines (Ariely and Wertenbroch 2002) or through binding contracts (Thaler and Benartzi 2004). In the small farm sector, Duflo et al. (2011) find that farmers are willing to join programs helping to overcome present-biasedness and make beneficial investments in time.

An additional factor that may also explain positive preferences for certain requirements is that the adoption of sustainability standards is often supported by farmer organizations through training or the provision of equipment and credits. Such measures and collective learning in the group may facilitate compliance and reduce possible psychological barriers for the individual. Sample farmers are mostly familiar with the general principles of sustainability standards and thus know that such support is often provided to certified farmers.

\subsubsection{Willingness to accept}

The model estimates can also be used to calculate farmers' willingness to accept (WTA) or willingness to pay (WTP) for the different attributes. Since the general idea is that the price premium compensates farmers for complying with particular requirements, we focus on the WTA. WTA estimates were obtained by dividing each attribute by the 
negative of the price coefficient. This approach is known as calculation in preference space. Results are shown in column (1) of table 19. Values may be biased when the price attribute is specified to be random (Hole and Kolstad 2012). Since we specified the price to be fixed, this should not be of concern here. However, as a robustness check we also calculated the WTA in WTP space, which is the preferred method with a random price specification (see column 2 in table 19). Results do not differ substantially.

WTA estimates are interpreted as the amount of money by which the price per kilogram had to be raised (or could be reduced) for farmers to opt for - or accept - a standard that includes the respective attribute. As indicated, the average coffee price during the time of our survey was about 2000 UGX. Regarding benefits, farmers would accept a price reduction of about 360 UGX if agricultural training was provided; about 200 UGX if training on gender was offered; and about 160 UGX if credits targeted at women were made available. This implies a range of 8-18 pecent of the average coffee price, which seems reasonable.

Table 19: Willingness to accept (WTA) estimates (UGX per kilogram of coffee)

\begin{tabular}{lcc}
\hline & $(1)$ & $(2)$ \\
& Mean WTA & Mean WTA \\
& calculated in preference space & calculated in WTP space \\
\hline Benefits & & \\
Agricultural training & -257 & -268 \\
Training on gender equality & {$[-311,-202]$} & {$[-262,-274]$} \\
& -199 & -179 \\
Credits for women & {$[-261,-136]$} & {$[-173,-186]$} \\
& -159 & -164 \\
Requirements & {$[-209,-109]$} & {$[-159,-169]$} \\
Pesticides prohibited & 159 & 152 \\
& {$[99,219]$} & {$[170,134]$} \\
Protective clothing & -192 & -179 \\
Only ripe cherries & {$[-251,-133]$} & {$[-177,-182]$} \\
No drying on soil & -378 & -394 \\
Record keeping & {$[-479,-277]$} & {$[-389,-399]$} \\
& {$[-142,-24]$} & -101 \\
Number of respondents & -117 & {$[-94,-107]$} \\
Note: WTA estimates were derived from mixed logit model parameter estimates (base specification), \\
using the delta method.
\end{tabular}


Regarding requirements, negative WTA estimates can be interpreted as farmers' willingness to invest in order to follow specified practices. Accordingly, the average farmer would be willing to invest 83 UGX in tarpaulins (per kilogram of coffee sold), used for drying coffee. Similarly, farmers would be willing to invest 380 UGX in hiring additional laborers for only picking ripe cherries. These values are above the actual costs for tarpaulins and labor, so the precise numbers should be interpreted with caution. However, price differences in the range of 80-400 UGX between high and low quality coffee are realistic. This supports our argument that farmers who are currently drying coffee on bare soil or pick unripe cherries may lack incentives to make investments in time - or may lack capital to make investments at all.

Finally, the average farmer is willing to invest about 190 UGX in protective clothing and about 120 UGX in record keeping. In contrast, farmers would only accept pesticide bans if the coffee price was raised by about 160 UGX, which could cover the cost of hiring additional laborers for weeding.

\subsubsection{Preference heterogeneity due to previous experience with standards}

We now explore some of the drivers of preference heterogeneity in more detail. In this subsection, we look at the role of farmers' actual experience with standards. In the next subsection, we focus on possible gender differences. In a first variation of the model's base specification we interact the ASC with the certification dummy. Results are shown in column (2) of table 18. The interaction term is insignificant, suggesting that actual experience with standards is not correlated with farmers' general preference for standards. Also when further disaggregating by type of standard (Fairtrade, Organic, or UTZ) no significant interaction effects with the ASC are found (column 3 of table 18).

However, experience with standards is correlated with preference heterogeneity for several attributes. We tested all possible interaction terms, but eventually excluded those that were individually or jointly insignificant. Column (4) of table 18 shows that Fairtrade and UTZ farmers have particularly strong preferences for the attribute 'record keeping'. We know that Fairtrade farmers are more likely to keep records (table 17). Thus, it is possible that larger preferences for record keeping among Fairtrade farmers are attributable to learning effects. On the other hand, it is also possible that farmers who have positive preferences for record keeping have self-selected into Fairtrade certification. Our data do not allow establishing the direction of causality. 
Further, we find that Organic farmers have less negative preferences for pesticide bans; the positive interaction term (0.50) in column (4) of table 18 is similar in magnitude to the negative 'pesticide prohibited' coefficient (-0.53), suggesting that Organic farmers would not require a significant price premium to be compensated for a pesticide ban. In reality, Organic farmers hardly use chemical pesticides anyway. Fairtrade farmers, on the other hand, have particularly negative attitudes towards a pesticide ban, which is in line with their higher actual pesticide use.

These results suggest that preferences are correlated with actual experience with sustainability standards. Farmers seem to have particularly positive preferences for requirements that they already comply with. As a robustness check, we estimate an additional model where we interact certification attributes with dummy variables for farmers' actual production and management practices. Results are shown in column (5) of table 18. Indeed, pesticide users have particularly negative attitudes towards pesticide bans, whereas record keepers have particularly positive preferences for record keeping. Similarly, farmers who have actually participated in agricultural or gender training have more positive preferences for such kinds of services.

\subsubsection{Gender-specific differences in preferences}

To explore possible gender differences, we specify a set of additional models, results of which are shown in table 20. In column (1), we interact the ASC with a simple female dummy. The interaction term is positive and significant, implying that women have a higher general preference for sustainability standards than men. In column (2), we further differentiate between female primary and female secondary decision-makers. While both interactions with the ASC produce positive estimates, only the coefficient for female secondary decision-makers is significant. This could mean that gender roles within the household and differences in decision-making power may be more relevant than the respondent's sex per se. Primary decision-makers are usually the ones who control revenues. Irrespective of their sex, they might be more concerned about the financial risk associated with the adoption of sustainability standards. In the worst case, investments may not pay off, contributing to more conservative attitudes. In contrast, female secondary decision-makers may not have a perfect overview of financial issues and may therefore give greater weight to the potential benefits of standards. 
Table 20: Mixed logit model estimates - Specifications to analyze gender differences

\begin{tabular}{|c|c|c|c|c|}
\hline & \multicolumn{3}{|c|}{ Full sample } & \multirow{2}{*}{$\begin{array}{r}\begin{array}{l}\text { Reduced } \\
\text { sample }\end{array} \\
(4)\end{array}$} \\
\hline Mean parameters & (1) & (2) & (3) & \\
\hline ASC (certification) & $1.27^{* * * *}(0.43)$ & $1.70^{* * *}(0.77)$ & $1.02^{* * *}(0.52)$ & $-0.59(0.77)$ \\
\hline Price premium (in 100 UGX) & $0.32^{* * *}(0.04)$ & $0.34^{* * *}(0.04)$ & $0.33^{* * * *}(0.04)$ & $0.26^{* * *}(0.06)$ \\
\hline Agricultural training $^{\text {a }}$ & $0.84^{* * *}(0.07)$ & $0.89^{* * *}(0.07)$ & $0.86^{* * *}(0.07)$ & $0.71^{* * * *}(0.09)$ \\
\hline Training on gender equality ${ }^{b}$ & $0.67^{* * *}(0.10)$ & $0.67^{* * *}(0.11)$ & $0.71^{* * * *}(0.11)$ & $0.57^{* * *}(0.14)$ \\
\hline Credits for women ${ }^{\mathrm{b}}$ & $0.51^{* * * *}(0.08)$ & $0.54^{* * * *}(0.08)$ & $0.42^{* * * *}(0.10)$ & $0.48^{* * * *}(0.11)$ \\
\hline Pesticides prohibited $^{\mathrm{c}}$ & $-0.52^{* * *}(0.10)$ & $-0.55^{* * *}(0.11)$ & $-0.56^{* * *}(0.11)$ & $-0.12(0.15)$ \\
\hline Protective clothing mandatory ${ }^{\mathrm{c}}$ & $0.70^{* * *}(0.09)$ & $0.65^{* * *}(0.09)$ & $0.69^{* * *}(0.09)$ & $0.24(0.16)$ \\
\hline Only ripe cherries ${ }^{\mathrm{d}}$ & $1.39^{* * *}(0.13)$ & $1.41^{* * *}(0.13)$ & $1.61^{* * *}(0.16)$ & $1.08^{* * *^{\prime}}(0.17)$ \\
\hline No drying on soil ${ }^{\mathrm{d}}$ & $0.35^{* * *}(0.10)$ & $0.28^{* * *}(0.10)$ & $0.29^{* * *}(0.10)$ & $0.24^{*}(0.13)$ \\
\hline Record keeping ${ }^{\mathrm{e}}$ & $0.43^{* * *}(0.07)$ & $0.42^{* * *}(0.07)$ & $0.44^{* * *}(0.07)$ & $0.22^{* *}(0.11)$ \\
\hline \multicolumn{5}{|l|}{ ASC and attribute interactions } \\
\hline ASC x Female & $0.93^{* *}(0.46)$ & & & \\
\hline ASC x Female primary & & $0.87(0.78)$ & $0.06(0.74)$ & \\
\hline ASC x Female secondary & & $1.02^{* *}(0.52)$ & $1.07^{* *}(0.51)$ & \\
\hline Credits for women $\mathrm{x}$ Female primary & & & $0.40^{*}(0.21)$ & \\
\hline Credits for women $\mathrm{x}$ Female secondary & & & $0.13(0.12)$ & \\
\hline Pesticides prohibited $\mathrm{x}$ Female primary & & & $0.39^{*}(0.22)$ & \\
\hline Only ripe cherries $\mathrm{x}$ Female secondary & & & $-0.38^{* * * *}(0.15)$ & \\
\hline No drying on soil $x$ Female primary & & & $0.31(0.24)$ & \\
\hline ASC $x$ Point estimate men & & & & $0.64^{* * *}(0.18)$ \\
\hline Pesticides proh. $\mathrm{x}$ Point estimate men & & & & $0.38^{* * *}(0.10)$ \\
\hline Protective clothing $\mathrm{x}$ Point estimate men & & & & $0.35^{* *}(0.15)$ \\
\hline Record keeping $\mathrm{x}$ Point estimate men & & & & $0.28^{*}(0.15)$ \\
\hline \multicolumn{5}{|l|}{ Standard deviation parameters } \\
\hline ASC & $0.41(039)$ & $0.16(0.52)$ & $0.60^{* *}(0.44)$ & $1.69^{* * *}(0.69)$ \\
\hline Agricultural training & $0.49^{* * *}(0.09)$ & $0.48^{* * *}(0.08)$ & $0.46^{* * *}(0.08)$ & $0.31^{* * *}(0.11)$ \\
\hline Training on gender equality & $0.51^{* * *}(0.14)$ & $0.61^{* * *}(0.14)$ & $0.55^{* * *}(0.13)$ & $0.63^{* * *}(0.21)$ \\
\hline Credits for women & $0.85^{* * *}(0.13)$ & $0.86^{* * *}(0.14)$ & $0.88^{* * *}(0.14)$ & $0.64^{* * *}(0.19)$ \\
\hline Pesticides prohibited & $1.58^{* * * *}(0.14)$ & $1.63^{* * * *}(0.15)$ & $1.59^{* * * *}(0.14)$ & $1.08^{* * * *}(0.17)$ \\
\hline Protective clothing mandatory & $0.90^{* * * *}(0.13)$ & $1.04^{* * * *}(0.15)$ & $0.97^{* * *}(0.16)$ & $0.62^{* * *}(0.20)$ \\
\hline Only ripe cherries & $1.12^{* * * *}(0.14)$ & $1.04^{* * * *}(0.13)$ & $1.02^{* * *}(0.12)$ & $0.88^{* * *}(0.18)$ \\
\hline No drying on soil & $0.97^{* * *}(0.14)$ & $0.94^{* * *}(0.16)$ & $0.94^{* * *}(0.14)$ & $0.82^{* * * *}(0.18)$ \\
\hline Record keeping & $0.70^{* * * *}(0.10)$ & $0.68^{* * * *}(0.10)$ & $0.67^{* * *}(0.09)$ & $0.53^{* * *}(0.17)$ \\
\hline Log Likelihood & -2384.38 & -2379.85 & -2370.72 & -880.98 \\
\hline Chi squared & $566.15^{* * *}$ & $574.37^{* * * *}$ & $571.35^{* * *}$ & $107.80^{* * *}$ \\
\hline Observations $(6 * 3 *$ No of respondents) & 12852 & 12852 & 12852 & 4698 \\
\hline \multicolumn{5}{|l|}{ Note: Standard errors in parentheses } \\
\hline \multicolumn{5}{|c|}{${ }^{a}$ Reference category is no agricultural training. } \\
\hline \multicolumn{5}{|c|}{${ }^{\mathrm{b}}$ Reference category is no gender policies (i.e. no training on gender equality and no credits for women). } \\
\hline \multirow{2}{*}{\multicolumn{5}{|c|}{$\begin{array}{l}{ }^{\mathrm{c}} \text { Reference category is no restrictions on pesticide use (i.e. pesticides allowed and protective clothing optional). } \\
\text { d Reference category is no quality requirements (i.e. picking only ripe cherries and drying on tarpaulins both } \\
\text { optional). }\end{array}$}} \\
\hline & & & & \\
\hline \multicolumn{5}{|c|}{ e Reference category is record keeping optional. } \\
\hline \multicolumn{5}{|c|}{$\begin{array}{l}{ }^{\mathrm{f}} \text { Only includes female respondents from households where a male respondent was also interviewed. } \\
{ }^{*} p<0.1,{ }^{* *} p<0.05,{ }^{* * *} p<0.01\end{array}$} \\
\hline
\end{tabular}


In a next step, we run a model with attribute-gender interaction terms, results of which are shown in column (3) of table 20. While we hypothesized women to have particularly strong preferences for gender policies, the interaction terms for 'training on gender equality' turned out to be insignificant (not shown). We conclude that such training is generally accepted by both male and female farmers. For the credit attribute, however, we do find gendered differences. Female primary decision-makers have a greater preference for credit access, probably because they are particularly suffering from constraints in formal credit markets.

We also find significant gender differences in terms of other attributes. Female primary decision-makers have less negative attitudes towards the prohibition of pesticides. This is probably related to the fact that only about 30 percent of the female-headed households in our sample actually used pesticides. As discussed above, non-users of pesticides tend to be more willing to accept a pesticide ban. Interestingly, compared to men, female secondary decision-makers have a less positive attitude towards the requirement to pick only ripe cherries. This is likely because spouses and other female members of the household are strongly involved in harvesting, so that more laborintensive quality requirements may increase their workload. In addition, female secondary decision-makers are often not involved in coffee sales, implying that they may be less aware of the financial benefit of supplying a higher-quality product.

So far, we have analyzed within-household heterogeneity only implicitly, by exploring differences due to gender and decision-making power. To analyze within-household heterogeneity more explicitly, we specify and estimate yet a different model. In column (4) of table 20, we restrict the sample to only those households in which we interviewed two respondents. Only in those households, we have data on preferences of both male and female members. In order to assess whether the preferences of male and female members in the same households are correlated, we use individual-level point estimates obtained from the model in column (1) of table 18 for male household members and include these as variables in explaining the preferences of female household members. More specifically, these point estimates are interacted with ASC and the standard attributes. Results in column (4) of table 20 reveal significantly positive interaction terms for the ASC (general standard preferences), for the requirements on pesticide use, and for record keeping, implying that male and female preferences for these attributes 
are positively correlated. ${ }^{30}$ For all other attributes, the interaction terms were found to be insignificant. The insignificant correlation between male and female preferences confirms the hypothesis that attitudes towards specific elements of sustainability standards are influenced by gender and decision-making power and may therefore vary within households.

\subsection{Conclusion}

A growing body of literature has analyzed whether sustainability standards are beneficial and feasible for smallholder farmers in developing countries, generally with mixed results. In this study, we have asked whether standards could be improved such that farm households benefit more. We have contributed to the literature by analyzing farmers' preferences for sustainability standards, and by employing a gendered research design. We have conducted a choice experiment with coffee growers in Uganda. Gender-disaggregated data have allowed us to explore possible differences in preferences between male and female decision-makers.

Sustainability certification is often understood as a scheme where farmers are compensated for complying with requirements that matter to consumers in developed countries. Our results suggest that a more differentiated consideration may be worthwhile. Some requirements are indeed seen by farmers as restrictions that are primarily associated with costs. A ban of chemical pesticides is one example of a requirement that the average farmer would only accept if she was compensated through a price premium. Even though not tested explicitly, we would expect negative attitudes also for bans on other productivity-enhancing inputs such as chemical fertilizers or certain types of seeds. This is consistent with Vlaeminck et al. (2015) who found negative preferences for some of the requirements associated with Organic farming.

However, other requirements may be beneficial for farmers, at least in the longer run. We have particularly analyzed requirements related to harvesting and post-harvest handling to improve coffee quality; record keeping to help manage farm resources more efficiently; and pesticide safety requirements to reduce health hazards for farmers and their families. Results show that farmers have positive attitudes towards such

\footnotetext{
${ }^{30}$ Note that the results in column (4) of table 20 cannot be compared directly with the other models in columns (1) to (3) because sample and model specification are different. This is why we confine interpretation of the estimates in column (4) to the interaction terms between male and female preferences.
} 
requirements and are willing to make related investments, even without an immediate output price premium. Sustainability standards that stipulate such practices are apparently seen by farmers as a welcome nudge to make investments that can pay off in the long run. In other words, standards could potentially be used more widely in the small farm sector to incentivize desirable investments and address possible issues of self-control and present-biasedness.

Model estimates also showed that preference heterogeneity exists. Positive preferences for particular requirements are more pronounced among farmers that already use related practices, for instance because they already adopted sustainability standards. This points at the important role of training, awareness building, and other support measures often provided by certified farmer organizations. Sustainability standards could also serve as a tool to reduce gender inequality. Gender equality can be promoted through specific training and awareness-building, services tailored to the needs of women (e.g. credit provision), or the introduction of gender-sensitive rules in farmer organizations. We showed that such attributes of sustainability standards are accepted and appreciated by male and female farmers alike. While some existing standards include related elements, a stronger focus on gender policies could be an interesting option to further explore.

The analysis also revealed that preferences for standards can be gender-specific. Female respondents have a stronger general preference for sustainability standards than male respondents, which is likely due to differences in the weighting of costs and benefits. We also identified preference heterogeneity with respect to certain attributes. Especially requirements related to harvesting and post-harvest handling to improve coffee quality were found to be less preferred by female spouses of male household heads, probably because these requirements can increase women's workload. Also other attributes of standards may affect male and female household members differently. Often, such differences are more related to gender-specific roles and tasks within the household rather than the sex of a person per se. Analysis of such nuances is not possible by comparing male-headed and female-headed households, but requires gender disaggregation of data within households, as was done here.

This is the first study that has analyzed gendered preferences for sustainability standards within farm households. Further work to refine the methodological approaches will be useful. It should be noted that - beyond actual experience with standards and gender, which were examined in this study - other factors can also contribute to preference heterogeneity and may be worth analyzing. Finally, we were only able to capture selected attributes of sustainability standards, even though many more are conceivable. 
Follow-up research should extend the focus and thus contribute to a deeper understanding of how sustainability standards can be designed for them to contribute to socially desirable developments in the small farm sector. This will require suitable combinations of quantitative and qualitative methods. 


\section{General conclusion}

Sustainability standards and certification schemes - such as Fairtrade, Organic, and UTZ - are gaining in importance for export crops produced by smallholder farmers in developing countries. Several studies have analyzed whether sustainability standards deliver on the promise to improve the livelihoods of poor farmers, with mixed results. This dissertation contributes to this literature in several ways. We have analyzed how different sustainability standards affect welfare outcomes at the household and individual levels. Unlike most previous studies that primarily looked at purely economic outcomes, we have examined welfare in terms of a broader set of social indicators, including child education, household nutrition, and gender equality. Further, based on choice experimental data, we have analyzed how sustainability standards could be improved to better address farmers' needs and preferences.

Previous studies have mainly focused on short-term economic effects of sustainability standards (e.g. prices, revenues, or income). Our results suggest that sustainability standards can have broader welfare implications, which may be difficult to capture when focusing on economic effects in a given year. Analyzing welfare effects beyond purely economic indicators is of particular importance for achieving the United Nation's Sustainable Development Goals (SDGs). For instance, our results suggest that sustainability standards can affect child education, household diets, and gender equality. Although we identify economic benefits (e.g. through higher prices) as one important pathway, we also identify other potential pathways.

We suggest that sustainability standards and certification should be understood as a package of requirements and benefits. The price premium is only one component of this package. Certified farmer organizations usually offer agricultural services to their members (e.g. extension, credits, access to farm inputs, and coffee processing). Indeed, our results indicate that certified farmers are significantly more likely to participate in agricultural trainings. Access to agricultural services can have important indirect economic effects. For instance, Fairtrade farmers in our sample are more likely to specialize on coffee and to use modern agricultural inputs. This may ultimately contribute to higher yield, higher incomes, and related broader welfare gains. Our results also show that farmers are, on average, willing to accept certification requirements related to better farm management (e.g. purchase of equipment to improve coffee quality or use of protective gear during pesticide application), even without being compensated through a price premium. This is because farmers are aware of the 
potential longer-term benefits associated with improved management practices. The combination of requirements related to farming practices and access to agricultural services seems to be a suitable avenue to incentivize desirable investments in the small farm sector.

Beyond farming, some sustainability standards also cover issues such as gender equality and child labor/education. Our results suggest that policies and rules on these issues can contribute to promoting related development outcomes (e.g. child education and gender equality). One key conclusion of this dissertation is that sustainability standards can have broader welfare implications, which cannot be captured by only looking at shortterm economic effects. Further, welfare effects are possibly not only related to the price premium for certified crops. From a development perspective, it is important to consider such broader welfare effects - and the underlying causal pathways.

Further, we show that effects of - and preferences for - sustainability standards can be heterogeneous, which should be considered in future impact studies. We find that gender and decision-making power matter, possibly because agricultural tasks and responsibilities are also allocated based on gender or decision-making power. For instance, female respondents have a stronger general preference for sustainability standards than male respondents, which is likely due to differences in the weighting of costs and benefits. Preference heterogeneity is also found for certain attributes. Especially requirements related to harvesting and post-harvest handling to improve coffee quality were found to be less preferred by female spouses of male household heads, probably because these requirements can increase women's workload. We also show that standards have heterogeneous effects on male household heads, female household heads, and female spouses. Further, sustainability standard may not only affect adult household members, but also children.

Our results further indicate that different type of sustainability standards can also have different types of effects. Differentiating between Fairtrade and Organic, we show that the effects of these two standards differ significantly: We find that Organic contributes to improved nutrition but has no effect on child education. For Fairtrade it is exactly the other way around. Dissimilar welfare implications are likely related to the specific design of each standard. Some differences are apparent - and likely matter for the overall effect of a given standard. For instance, Organic bans chemical pesticides completely, while Fairtrade only prohibits particularly harmful pesticides. We show that farmers have negative preferences for the ban of chemical pesticides, because such input restrictions increase the demand for manual labor for pest control (e.g. weeding). 
Other differences in the design of standards are less obvious - but may still be important. Written documents on specific standards often include components and details that can cover dozens of pages. Disentangling the effect of each component is probably impossible. Indeed, impact studies typically assess the effect of a given standard rather than the effect of specific components. As it remains unclear how different components contribute to the overall effect, results regarding one standard are not generalizable to other standards, as we show with our results.

Although not specifically analyzed here, the effect of sustainability standards probably also depends on context-specific factors. Standards encompass both mandatory certification requirement and recommended practices. Mandatory certification requirements may affect farmers in different ways, given local conditions. For instance, a ban of pesticides may not affect farmers who do not use chemical inputs anyways, but would affect farmers who do. Recommended practices may be implemented in different ways - or not at all, given local conditions. For instance, we have looked at the effect of gender policies, which largely fall under the category of recommended practices. The Fairtrade and UTZ farmer organizations in our sample chose to implement training workshops to promote awareness on gender equality. Other farmer organizations may introduce special programs targeting women farmers' needs instead. And yet other farmer organizations may not implement any of the recommended gender policies at all. Such differences in the implementation of standards in a specific context will likely affect certification outcomes.

Choices of farmer organizations on implementation may also matter beyond gender policies. For instance, after close interactions with the Fairtrade farmer organization leadership, we were convinced they are committed to serve and benefit their members. This commitment is reflected in the wide range of services provided by the Fairtrade farmer organization and the considerate use of the Fairtrade premium for developmentpromoting investments (e.g. establishment of an input shop, credit schemes, and processing facilities). Shortly after our survey, the Fairtrade farmer organization was even awarded a prize by Fairtrade International. Other farmer organizations may not (be able to) implement such a wide range of services to their members. Differences in the management quality of certified farmer organizations, and specific choices made, possibly affect certification outcomes. Given such location-specific factors, our concrete numerical findings cannot be broadly generalized. But the conceptual insights into welfare-enhancing potentials of sustainability standards, causal pathways, heterogeneous effects, and smallholder preferences will hopefully be useful and 
contribute to the research direction also beyond the concrete empirical setting in Uganda.

Although the concrete empirical findings cannot be generalized, they are in line with many previous studies, suggesting that sustainability standards can be beneficial for farmers who are able to adopt them. However, various studies also draw less positive conclusions. Thus, based on the existing body of literature, sustainability standards should neither be promoted as a silver bullet for poverty reduction, nor should they be seen as an empty promise. A remaining research task is to further disentangle the contextual conditions under which specific standards promote sustainability goals. This may require cross-country comparisons.

A few additional limitations have to be mentioned. First, impact studies presented in this dissertation disregard local spillover effects (e.g. how does the adoption of standards by some farmers affect neighboring farmers and farmer organizations that do not (cannot) adopt?). Future research into this direction may be useful. Second, in spite of the fairly comprehensive data collected, some data and methodological challenges remain. We use observational data for our impact studies, which is generally associated with possible issues of self-selection bias. We employed various econometric techniques - such as fixed effects panel data models, instrumental variable approaches, and entropy balancing - to reduce such bias. However, all of these approaches have their shortcomings. Impact evaluations with randomized controlled trials might address some of these shortcomings (and possibly be associated with other drawbacks), but have not yet been carried out for sustainability standards. Finally, although we have considered various economic and social effects, sustainability standards also have environmental objectives, which we did not consider here. Follow-up research should address these limitations. 


\section{References}

Alderman, H., P.-A. Chiappori, L. Haddad, J. Hoddinott, and R. Kanbur. 1995. Unitary versus collective models of the household: Is it time to shift the burden of proof. World Bank Research Observer 10(1): 1-19.

Alkire, S., R. Meinzen-Dick, A. Peterman, A. Quisumbing, G. Seymour, and A. Vaz. 2013. The Women's Empowerment in Agriculture Index. World Development 52: $71-91$.

Anderson, C. L., T. W. Reynolds, and M. Kay Gugerty. 2017. Husband and wife perspectives on farm household decision-making authority and evidence on intrahousehold accord in rural Tanzania. World Development 90: 169-183.

Ariely, D. and K. Wertenbroch. 2002. Procrastination, deadlines, and performance: Self-control by precommitment. Psychological Science 13(3): 219-224.

Arnould, E. J., A. Plastina, and D. Ball. 2009. Does Fair Trade deliver on its core value proposition? Effects on income, educational attainment, and health in three countries. Journal of Public Policy \& Marketing 28(2): 186-201.

Asfaw, S., D. Mithöfer, and H. Waibel. 2010. Agrifood supply chain, private-sector standards, and farmers' health: Evidence from Kenya. Agricultural Economics 41(34): 251-263.

Ayuya, O. I., E. O. Gido, H. K. Bett, J. K. Lagat, A. K. Kahi, and S. Bauer. 2015. Effect of certified Organic production systems on poverty among smallholder farmers: Empirical evidence from Kenya. World Development 67: 27-37.

Bacon, C. 2005. Confronting the coffee crisis: Can Fair Trade, Organic, and specialty coffees reduce small-scale farmer vulnerability in Northern Nicaragua? World Development 33(3): 497-511.

Bacon, C. M. 2010. A spot of coffee in crisis: Nicaraguan smallholder cooperatives, Fair Trade networks, and gendered empowerment. Latin American Perspectives 37(2): 50-71.

Bacon, C. M., V. Ernesto Méndez, M. E. F. Gómez, D. Stuart, and S. Raúl Díaz Flores. 2008. Are sustainable coffee certifications enough to secure farmer livelihoods? The 
Millenium Development Goals and Nicaragua's Fair Trade cooperatives. Globalizations 5(2): 259-274.

Bassett, T. J. 2010. Slim pickings: Fairtrade cotton in West Africa. Geoforum 41(1): $44-55$.

Becchetti, L., S. Castriota, and M. Michetti. 2013. The effect of Fair Trade affiliation on child schooling: Evidence from a sample of Chilean honey producers. Applied Economics 45(25): 3552-3563.

Becchetti, L. and M. Costantino. 2008. The effects of Fair Trade on affiliated producers: An impact analysis on Kenyan farmers. World Development 36(5): 823-842.

Bech, M. and D. Gyrd-Hansen. 2005. Effects coding in discrete choice experiments. Health Economics 14(10): 1079-1083.

Beuchelt, T. D. and M. Zeller. 2011. Profits and poverty: Certification's troubled link for Nicaragua's Organic and Fairtrade coffee producers. Ecological Economics 70(7): 1316-1324.

Black, R. E., L. H. Allen, Z. A. Bhutta, L. E. Caulfield, M. de Onis, M. Ezzati, C. Mathers, and J. Rivera. 2008. Maternal and child undernutrition: Global and regional exposures and health consequences. The Lancet 371(9608): 243-260.

Blackman, A. and M. A. Naranjo. 2012. Does eco-certification have environmental benefits? Organic coffee in Costa Rica. Ecological Economics 83: 58-66.

Bolwig, S. 2012. Poverty and gender effects of smallholder Organic contract farming in Uganda. USSP Working Paper 8, International Food Policy Research Institute (IFPRI). Washington, D.C.

Bolwig, S., P. Gibbon, and S. Jones. 2009. The economics of smallholder Organic contract farming in tropical Africa. World Development 37(6): 1094-1104.

Bonnan-White, J., A. Hightower, and A. Issa. 2013. Of couscous and occupation: A case study of women's motivations to join and participate in Palestinian Fair Trade cooperatives. Agriculture and Human Values 30(3): 337-350.

Chambolle, C. and S. Poret. 2013. When Fairtrade contracts for some are profitable for others. European Review of Agricultural Economics 40(5): 835-871. 
Chege, C. G., C. I. Andersson, and M. Qaim. 2015. Impacts of supermarkets on farm household nutrition in Kenya. World Development 72: 394-407.

Chiputwa, B. and M. Qaim. 2016. Sustainability standards, gender, and nutrition among smallholder farmers in Uganda. Journal of Development Studies 52(9): 1241-1257.

Chiputwa, B., D. J. Spielman, and M. Qaim. 2015. Food standards, certification, and poverty among coffee farmers in Uganda. World Development 66: 400-412.

Deere, C. D. and C. R. Doss. 2006. The gender asset gap: What do we know and why does it matter? Feminist Economics 12(1-2): 1-50.

Doss, C. 2013. Intrahousehold bargaining and resource allocation in developing countries. World Bank Research Observer 28(1): 52-78.

Doss, C. R. 2001. Designing agricultural technology for African women farmers: Lessons from 25 years of experience. World Development 29(12): 2075-2092.

Doss, C. R. 2002. Men's crops? Women's crops? The gender patterns of cropping in Ghana. World Development 30(11): 1987-2000.

Doss, C. R., C. D. Deere, A. D. Oduro, and H. Swaminathan. 2014. The gender asset and wealth gaps. Development 57(3-4): 400-409.

Duflo, E., M. Kremer, and J. Robinson. 2011. Nudging farmers to use fertilizer: Theory and experimental evidence from Kenya. American Economic Review 101(6): 23502390.

Duflo, E. and C. Udry. 2004. Intrahousehold resource allocation in Cote d'Ivoire: Social norms, separate accounts and consumption choices. Cambridge, MA: National Bureau of Economic Research.

Ecker, O. and M. Qaim. 2011. Analyzing nutritional impacts of policies: An empirical study for Malawi. World Development 39(3): 412-428.

Elder, S. D., J. Lister, and P. Dauvergne. 2013. Big retail and sustainable coffee: A new development studies research agenda. Progress in Development Studies 14(1): 7790. 
Elder, S. D., H. Zerriffi, and P. Le Billon. 2012. Effects of Fair Trade certification on social capital: The case of Rwandan coffee producers. World Development 40(11): 2355-2367.

Elder, S. D., H. Zerriffi, and P. Le Billon. 2013. Is Fairtrade certification greening agricultural practices? An analysis of Fairtrade environmental standards in Rwanda. Journal of Rural Studies 32: 264-274.

Fairtrade International. 2009. Gender Strategy 2016-2020: Transforming Equal Opportunity, Access and Benefits for All, Fairtrade International. Bonn.

Fairtrade International. 2011a. Fairtrade Standard for Small Producer Organizations, Fairtrade International. Bonn.

Fairtrade International. 2011b. Official homepage: Fairtrade International. www.fairtrade.net, Accessed October 13, 2016.

Fairtrade International. 2015. Scope and Benefits of Fairtrade, Fairtrade International. Bonn.

FAO. 2011. The State of Food and Agriculture: Women in Agriculture - Closing the Gender Gap for Development. Rome: Food and Agriculture Organization of the United Nations (FAO).

FAO. 2015. Official homepage: Food and Agriculture Organization of the United Nations (FAO): FAOSTAT. http://faostat.fao.org/, Accessed October 25, 2016.

FAO, WHO, and UNU. Human Energy Requirements: Report of a Joint FAO/WHO/UNU Expert Consultation: Rome, 17-24 October 2001. FAO food and nutrition technical report series, 1813-3923, United Nations University (UNU), World Health Organization (WHO) and Food and Agriculture Organization of the United Nations (FAO). Rome.

FiBL and IFOAM. 2016. The World of Organic Agriculture: Statistics and Emergining Trends 2016, Reseach Institute of Organic Agriculture (FiBL) and International Federation of Organic Agriculture Movements (IFOAM). Frick, Bonn.

Fischer, E. and M. Qaim. 2012. Gender, agricultural commercialization, and collective action in Kenya. Food Security 4(3): 441-453. 
Freier, R., M. Schumann, and T. Siedler. 2015. The earnings returns to graduating with honors — Evidence from law graduates. Labour Economics 34: 39-50.

Gitter, S. R., J. G. Weber, B. L. Barham, M. Callenes, and J. Lewis Valentine. 2012. Fair Trade-Organic coffee cooperatives, migration, and secondary schooling in southern Mexico. Journal of Development Studies 48(3): 445-463.

Grunert, K. G., S. Hieke, and J. Wills. 2014. Sustainability labels on food products: Consumer motivation, understanding and use. Food Policy 44: 177-189.

Hainmueller, J. 2012. Entropy balancing for causal effects: A multivariate reweighting method to produce balanced samples in observational studies. Political Analysis 20(1): 25-46.

Handschuch, C. and M. Wollni. 2015. Traditional food crop marketing in Sub-Saharan Africa: Does gender matter? Journal of Development Studies 52(3): 343-359.

Handschuch, C., M. Wollni, and P. Villalobos. 2013. Adoption of food safety and quality standards among Chilean raspberry producers - Do smallholders benefit? Food Policy 40: 64-73.

Hensher, D. A., J. M. Rose, and W. H. Greene. 2005. Applied Choice Analysis: A Primer. Cambridge: Cambridge University Press.

Hirano, K., G. W. Imbens, and G. Ridder. 2003. Efficient estimation of average treatment effects using the estimated propensity score. Econometrica 71(4): 11611189.

Hirano, K., G. W. Imbens, G. Ridder, and D. B. Rubin. 2001. Combining panel data sets with attrition and refreshment samples. Econometrica 69(6): 1645-1659.

Hoddinott, J. and L. Haddad. 1995. Does female income share influence household expenditures? Evidence from Côte d'ivoire. Oxford Bulletin of Economics and Statistics 57(1): 77-96.

Hole, A. R. and J. Riise Kolstad. 2012. Mixed logit estimation of willingness to pay distributions: A comparison of models in preference and WTP space using data from a health-related choice experiment. Empirical Economics 42(2): 445-469. 
Holmes, T. P. and W. L. Adamowicz. 2003. Attribute-based Methods. In P. A. Champ, K. J. Boyle and T. C. Brown (eds), A Primer on Nonmarket Valuation. Dordrecht: Springer Netherlands, 171-219.

Hoogland, C. T., J. de Boer, and J. J. Boersema. 2007. Food and sustainability: Do consumers recognize, understand and value on-package information on production standards? Appetite 49(1): 47-57.

Hope, R., M. Borgoyary, and C. Agarwal. 2008. Smallholder preferences for agrienvironmental change at the Bhoj wetland, India. Development Policy Review 26(5): 585-602.

Hotz, C., A. Lubowa, and C. Sison. 2012. A Food Composition Table for Central and Eastern Uganda. HarvestPlus Technical Monograph 9, International Food Policy Research Institute (IFPRI) and International Center for Tropical Agriculture (CIAT). Washington, DC, Cali.

Huang, H.-C. and C.-C. Yeh. 2014. Inflation targeting on unemployment rates: A quantile treatment effect approach. Applied Economics Letters 21(7): 453-458.

Hutchens, A. 2010. Empowering women through Fair Trade? Lessons from Asia. Third World Quarterly 31(3): 449-467.

Ibanez, M. and A. Blackman. 2016. Is eco-certification a win-win for developing country agriculture? Organic coffee certification in Colombia. World Development 82: 14-27.

Ibnu, M., P. Glasbergen, A. Offermans, and B. Arifin. 2015. Farmer preferences for coffee certification: A conjoint analysis of the Indonesian smallholders. Journal of Agricultural Science 7(6): 20-35.

ICO. 2016. Official homepage: International Coffee Organization (ICO). http://www.ico.org/.

IFOAM. 2014. The IFOAM Norms for Organic Production and Processing, International Federation of Organic Agriculture Movements (IFOAM). Bonn.

ITC. 2011. Trends in the Trade of Certified Coffees, International Trade Centre (ITC). Geneva. 
ITC. 2012. Uganda National Export Strategy: Coffee Sector Export Strategy Update 2012-2017, International Trade Centre (ITC). Geneva.

ITC. 2015. The State of Sustainable Markets: Statistics and Emerging Trends 2015, International Trade Centre (ITC). Geneva.

ITC. 2016. Standards Map: International Trade Centre (ITC). http://www.standardsmap.org/identify, Accessed September 20, 2016.

Janssen, M. A. and J. M. Anderies. 2013. A multi-method approach to study robustness of social-ecological systems: The case of small-scale irrigation systems. Journal of Institutional Economics 9(04): 427-447.

Jena, P. R., B. B. Chichaibelu, T. Stellmacher, and U. Grote. 2012. The impact of coffee certification on small-scale producers' livelihoods: A case study from the Jimma Zone, Ethiopia. Agricultural Economics 43(4): 429-440.

Jena, P. R., T. Stellmacher, and U. Grote. 2017. Can coffee certification schemes increase incomes of smallholder farmers? Evidence from Jinotega, Nicaragua. Environment, Development and Sustainability 19(1): 45-66.

Johnson, N. L., C. Kovarik, R. Meinzen-Dick, J. Njuki, and A. Quisumbing. 2016. Gender, assets, and agricultural development: Lessons from eight projects. World Development 83: 295-311.

Jones, A. D., A. Shrinivas, and R. Bezner-Kerr. 2014. Farm production diversity is associated with greater household dietary diversity in Malawi: Findings from nationally representative data. Food Policy 46: 1-12.

Jones, S. and P. Gibbon. 2011. Developing agricultural markets in Sub-Saharan Africa: Organic cocoa in rural Uganda. Journal of Development Studies 47(10): 1595-1618.

Kabeer, N. 1999. Resources, agency, achievements: Reflections on the measurement of women's empowerment. Development \& Change 30(3): 435-464.

Karki, S. K., P. R. Jena, and U. Grote. 2016. Fair Trade certification and livelihoods: A panel data analysis of coffee-growing households in India. Agricultural and Resource Economics Review 45(03): 436-458. 
Kersting, S. and M. Wollni. 2012. New institutional arrangements and standard adoption: Evidence from small-scale fruit and vegetable farmers in Thailand. Food Policy 37(4): 452-462.

Klasen, S. 2000. Measuring poverty and deprivation in South Africa. Review of Income and Wealth 46(1): 33-58.

Kleemann, L. and A. Abdulai. 2013. Organic certification, agro-ecological practices and return on investment: Evidence from pineapple producers in Ghana. Ecological Economics 93: 330-341.

Kleemann, L., A. Abdulai, and M. Buss. 2014. Certification and access to export markets: Adoption and return on investment of Organic-certified pineapple farming in Ghana. World Development 64: 79-92.

Kloos, J. and F. G. Renaud. 2014. Organic cotton production as an adaptation option in north-west Benin. Outlook on Agriculture 43(2): 91-100.

Lee, J., G. Gereffi, and J. Beauvais. 2012. Global value chains and agrifood standards: challenges and possibilities for smallholders in developing countries. Proceedings of the National Academy of Sciences of the United States of America 109(31): 1232612331.

Loconto, A. 2015. Can certified-tea value chains deliver gender equality in Tanzania? Feminist Economics 21(3): 191-215.

Lyon, S. 2008. We want to be equal to them: Fair-trade coffee certification and gender equity within organizations. Human Organization 67(3): 258-268.

Lyon, S., J. A. Bezaury, and T. Mutersbaugh. 2010. Gender equity in Fairtrade-Organic coffee producer organizations: Cases from Mesoamerica. Geoforum 41(1): 93-103.

Maertens, M. and J. F.M. Swinnen. 2009. Trade, standards, and poverty: Evidence from Senegal. World Development 37(1): 161-178.

Maertens, M. and J. F.M. Swinnen. 2012. Gender and modern supply chains in developing countries. Journal of Development Studies 48(10): 1412-1430.

Malapit, H. J. L. and A. R. Quisumbing. 2015. What dimensions of women's empowerment in agriculture matter for nutrition in Ghana? Food Policy 52: 54-63. 
Marcus, J. 2013. The effect of unemployment on the mental health of spouses Evidence from plant closures in Germany. Journal of health economics 32(3): 546558.

Marenya, P., V. H. Smith, and E. Nkonya. 2014. Relative preferences for soil conservation incentives among smallholder farmers: Evidence from Malawi. American Journal of Agricultural Economics 96(3): 690-710.

McFadden, D. L. 1973. Conditional Logit Analysis of Qualitative Choice Behavior. In P. Zarembka (ed.), Frontiers in Econometrics. New York: Academic Press, 105142.

Meemken, E.-M., P. C. Veettil, and M. Qaim. 2016. Small farmers' preferences for the design of certification schemes: Does gender matter? GlobalFood Discussion Paper 83, University of Göttingen.

Méndez, V. E., C. M. Bacon, and R. Cohen. 2013. Agroecology as a transdisciplinary, participatory, and action-oriented approach. Sustainable Food Systems 37(1): 3-18.

Méndez, V. E., C. M. Bacon, M. Olson, K. S. Morris, and A. Shattuck. 2010. Agrobiodiversity and shade coffee smallholder livelihoods: A review and synthesis of ten years of research in Central America. The Professional Geographer 62(3): $357-376$.

Méndez, V. E., C. M. Bacon, M. Olson, S. Petchers, D. Herrador, C. Carranza, L. Trujillo, C. Guadarrama-Zugasti, A. Cordón, and A. Mendoza. 2010. Effects of Fair Trade and Organic certifications on small-scale coffee farmer households in Central America and Mexico. Renewable Agriculture and Food Systems 25(03): 236-251.

Mitiku, F., Y. de Mey, J. Nyssen, and M. Maertens. 2017. Do private sustainability standards contribute to income growth and poverty alleviation? A comparison of different coffee certification schemes in Ethiopia. Sustainability 9(2): 246.

Neuenkirch, M. and F. Neumeier. 2016. The impact of US sanctions on poverty. Journal of Development Economics 121: 110-119.

Njuki, J., S. Kaaria, A. Chamunorwa, and W. Chiuri. 2011. Linking smallholder farmers to markets, gender and intra-household dynamics: Does the choice of commodity matter? European Journal of Development Research 23(3): 426-443. 
Ochieng, D. O., P. C. Veettil, and M. Qaim. 2017. Farmers' preferences for supermarket contracts in Kenya. Food Policy 68: 100-111.

Omidvar, V. and K. Giannakas. 2015. The effects of fair trade on coffee growers: A framework and analysis. Agricultural Economics 46(S1): 29-39.

Orr, A., T. Tsusaka, S. H. Kee-Tui, and H. Msere. 2016. What do we mean by 'women's crops'? Commercialisation, gender and the power to name. Journal of International Development 28(6): 919-937.

Parvathi, P. and H. Waibel. 2016. Organic agriculture and Fair Trade: A happy marriage? A case study of certified smallholder black pepper farmers in India. World Development 77: 206-220.

Pirracchio, R., M. Resche-Rigon, and S. Chevret. 2012. Evaluation of the propensity score methods for estimating marginal odds ratios in case of small sample size. BMC Medical Research Methodology 12: 70.

Potts, J., M. Lynch, A. Wilkings, G. A. Huppé, M. Cunningham, and V. Anand Voora. 2014. The State of Sustainability Initiatives Review 2014: Standards and the Green Economy. Winnipeg, Manitoba, London, Beaconsfield, Quebec: International Institute for Sustainable Development (IISD), International Institute for Environment and Development; Canadian Electronic Library.

Quisumbing, A. R., D. Rubin, C. Manfre, E. Waithanji, M. van den Bold, D. Olney, N. Johnson, and R. Meinzen-Dick. 2015. Gender, assets, and market-oriented agriculture: Learning from high-value crop and livestock projects in Africa and Asia. Agriculture and Human Values 32(4): 705-725.

Ranjan Jena, P. and U. Grote. 2016. Fairtrade certification and livelihood impacts on small-scale coffee producers in a tribal community of India. Applied Economic Perspectives and Policy: ppw006.

Rao, E. J. and M. Qaim. 2011. Supermarkets, farm household income, and poverty: Insights from Kenya. World Development 39(5): 784-796.

Raynolds, L. T. 2017. Fairtrade labour certification: The contested incorporation of plantations and workers. Third World Quarterly: 1-20. 
Raynolds, L. T., D. Murray, and A. Heller. 2007. Regulating sustainability in the coffee sector: A comparative analysis of third-party environmental and social certification initiatives. Agriculture and Human Values 24(2): 147-163.

Ruben, R. and R. Fort. 2012. The impact of Fair Trade certification for coffee farmers in Peru. World Development 40(3): 570-582.

Ruben, R. and G. Zuniga. 2011. How standards compete: Comparative impact of coffee certification schemes in Northern Nicaragua. Supply Chain Management: An International Journal 16(2): 98-109.

Schipmann, C. and M. Qaim. 2011. Supply chain differentiation, contract agriculture, and farmers' marketing preferences: The case of sweet pepper in Thailand. Food Policy 36(5): 667-677.

Sen, D. 2014. Fair Trade vs. swaccha vyāpār: Women's activism and transnational justice regimes in Darjeeling, India. Feminist Studies 40(2): 444-472.

Sibhatu, K. T., V. V. Krishna, and M. Qaim. 2015. Production diversity and dietary diversity in smallholder farm households. Proceedings of the National Academy of Sciences of the United States of America 112(34): 10657-10662.

Swinnen, J. and A. Vandeplas. 2011. Rich consumers and poor producers: Quality and rent distribution in global value chains. Journal of Globalization and Development 2(2).

Takahashi, R. and Y. Todo. 2017. Coffee certification and forest quality: Evidence from a wild coffee forest in Ethiopia. World Development 92: 158-166.

Tallontire, A., M. Opondo, and V. Nelson. 2014. Contingent spaces for smallholder participation in GlobalGAP: Insights from Kenyan horticulture value chains. Geographical Journal 180(4): 353-364.

Terstappen, V., L. Hanson, and D. McLaughlin. 2013. Gender, health, labor, and inequities: A review of the fair and alternative trade literature. Agriculture and Human Values 30(1): 21-39.

Thaler, R. H. and S. Benartzi. 2004. Save More Tomorrow ${ }^{\mathrm{TM}}$ : Using behavioral economics to increase employee saving. Journal of Political Economy 112: 164187. 
Train, K. 2009. Discrete Choice Methods with Simulation. Cambridge: New York.

UBOS. 2010. Uganda Census of Agriculture 2008/2009, Uganda Bureau of Statistics (UBOS) and Ministry of Agriculture, Animal Industry and Fisheries. Kampala.

UBOS. 2016. Statistical Abstract, Uganda Bureau of Statistics (UBOS). Kampala.

UCDA. 2016. Official homepage: Uganda Coffee Development Authority (UCDA). http://ugandacoffee.go.ug/general-2/, Accessed September 20, 2016.

UIA. 2016. Official homepage: Uganda Investment Authority (UIA). http://www.ugandainvest.go.ug/coffee-sector-profile/, Accessed September 20, 2016.

UN. 2016. Official homepage United Nations (UN): Sustainable Development Goals. https://sustainabledevelopment.un.org/sdgs, Accessed December 11, 2016.

UTZ. 2015a. Core Code of Conduct: For Group and Multi-Group Certification, UTZ. Amsterdam.

UTZ. 2015b. Official homepage: UTZ. www.utz.org, Accessed November 28, 2016.

UTZ. 2015c. Positioning Paper: Gender, UTZ. Amsterdam.

Valkila, J. 2009. Fair Trade Organic coffee production in Nicaragua - Sustainable development or a poverty trap? Ecological Economics 68(12): 3018-3025.

Valkila, J. and A. Nygren. 2010. Impacts of Fair Trade certification on coffee farmers, cooperatives, and laborers in Nicaragua. Agriculture and Human Values 27(3): 321333.

van Rijsbergen, B., W. Elbers, R. Ruben, and S. N. Njuguna. 2016. The ambivalent impact of coffee certification on farmers' welfare: A matched panel approach for cooperatives in central Kenya. World Development 77: 277-292.

Verhofstadt, E. and M. Maertens. 2014. Smallholder cooperatives and agricultural performance in Rwanda: Do organizational differences matter? Agricultural Economics 45(S1): 39-52.

Vlaeminck, P., L. Vranken, G. van den Broeck, K. Vande Velde, K. Raymaekers, and M. Maertens. 2015. Farmers' preferences for Fair Trade contracting in Benin. 
Bioeconomics Working Paper Series 225931, Katholieke Universiteit Leuven. Leuven.

von Braun, J. and Kennedy, E. T. (eds). 1994. Agricultural Commercialization, Economic Development, and Nutrition. Baltimore, MD: Johns Hopkins University Press.

Ward, P. S., D. L. Ortega, D. J. Spielman, and V. Singh. 2014. Heterogeneous demand for drought-tolerant rice: Evidence from Bihar, India. World Development 64: 125139.

Weber, J. G. 2011. How much more do growers receive for Fair Trade-organic coffee? Food Policy 36(5): 678-685.

Wollni, M. and M. Zeller. 2007. Do farmers benefit from participating in specialty markets and cooperatives? The case of coffee marketing in Costa Rica1. Agricultural Economics 37(2-3): 243-248.

Wooldridge, J. M. 2007. Inverse probability weighted estimation for general missing data problems. Journal of Econometrics 141(2): 1281-1301.

World Bank. 2016. Official homepage: World Bank. www.worldbank.org, Accessed May 3, 2016.

Yu, J. and Z. Bouamra-Mechemache. 2015. Production standards, competition and vertical relationship. European Review of Agricultural Economics 43(1): 79-111. 
General appendix 
“Dear Respondent! My name is.... I represent a survey team from the University of Goettingen (Germany). We are following up on respondents who were interviewed in 2012. The objective of this study is to learn more about farmer participation in coffee certification schemes and the adoption of mobile phone technologies. Your cooperation in answering the questions is very much appreciated. Please answer all questions as accurately and truthfully as possible. Your responses will be COMPLETELY CONFIDENTIAL - that is we assure that your individual responses will not be disclosed to anyone; the answers will be used for research purpose only. The survey would only take about 2.5 hrs. THANK YOU!! For this interview, we would like to talk to the two primary decision makers within this HH (over 18 years, one male and one female). The primary decision makers are referred to as the persons responsible for decision making both in economic and social terms. The interview has two parts. The first part should be answered by the person we interviewed in 2012. The second part should be answered by both the primary and secondary decision maker SEPARATELY.

\subsection{Enumerator}

\begin{tabular}{|c|c|c|c|}
\hline 1.1 Name & 1.2 Date of interview & 1.3 Time interview started & 1.4 Time interview ended \\
\hline & $/ 2015$ & & \\
\hline
\end{tabular}

\subsection{HH location}

\begin{tabular}{|l|l|l|l|l|}
\hline 2.1 District (1=Luwero 2=Masaka/Bukomansimbi) & 2.2 County & 2.3 Sub county & 2.4 Parish \\
\hline & & & \\
\hline
\end{tabular}

\begin{tabular}{|c|c|c|c|}
\hline 2.6 GPS_north & $2.7 \mathrm{GPS}:$ south & 2.8 GPS_east & 2.9 Altitude \\
\hline
\end{tabular}

3.1 Who is the primary decision maker (HHD) within this HH? Name:

3.2 Is this person available for an interview? ___ ( $0=$ No $1=$ Yes $) 3.3$ If no, why not?

(1=Temporarily away $2=$ Absent from home at least 6 month $3=$ Refused $\quad 4=0$ ther (specify))

4.1 Who is the secondary decision maker within this HH (usually spouse)? Name:

4.2 Is this person available for an interview?

(1=Temporarily away $2=$ Absent from home at least 6 month $3=$ Refused $4=0$ ther (specify))

5.0 Respondent interviewed in 2012

\begin{tabular}{|l|l|l|l|l|}
\hline 5.1 Name & 5.2 Gender (1=Male 2=Female) & 5.3 Phone number & $\begin{array}{l}5.4 \text { Was this person HHD in 2012? (0=No } \\
1=\text { Yes) }\end{array}$ & \\
\hline & & & \\
\hline
\end{tabular}

6.1 Who is the first respondent? (Preferably, this should be the respondent interviewed in 2012. This person should answer part 1 and part 2 of the questionnaire)

6.2 Is the respondent of 2012 available to answer part 1 and 2 of the questionnaire? _ ( $0=$ No $1=\mathrm{YES} \rightarrow$ make sure information above is correct and update it if necessary; then move to Q7).

6.3 If no, why is the respondent of 2012 not available for an interview? (1=Temporarily away $2=$ Absent from home at least 6 months $3=$ Death $4=0$ ther (specify))

\begin{tabular}{|c|c|c|c|}
\hline 6.4 Name of the first respondent & $\begin{array}{l}6.5 \text { Gender (1=Male } \\
2=\text { Female) }\end{array}$ & 6.6 Phone number & $\begin{array}{l}6.7 \text { This person is the } 1=\text { Primary decision maker } \\
2=\text { Secondary decision maker } 3=\text { Third decision maker }\end{array}$ \\
\hline
\end{tabular}

7.0 Who is the second respondent? (This person should answer part 2 of the questionnaire)

\begin{tabular}{|l|l|l|l|}
\hline 7.1 Name of the second respondent & $\begin{array}{l}7.2 \text { Gender (1=Male 2=Female) CANNOT } \\
\text { be the same as the first respondent! }\end{array}$ & $\begin{array}{l}7.3 \text { Phone number } \\
\text { 2=Secondary decision maker 3=Third decision maker }\end{array}$ & \\
\hline & & \\
\hline
\end{tabular}


SECTION 1 HOUSEHOLD INFORMATION (E)

1 How many people normally ${ }^{1}$ live and eat their meals in this HH (including servants and other workers). Tenants who pay rent are NOT considered members. Number of HH members:

2.0 Please provide the list of names of each member in this HH starting with the head, spouse, and his /her children in order of age.

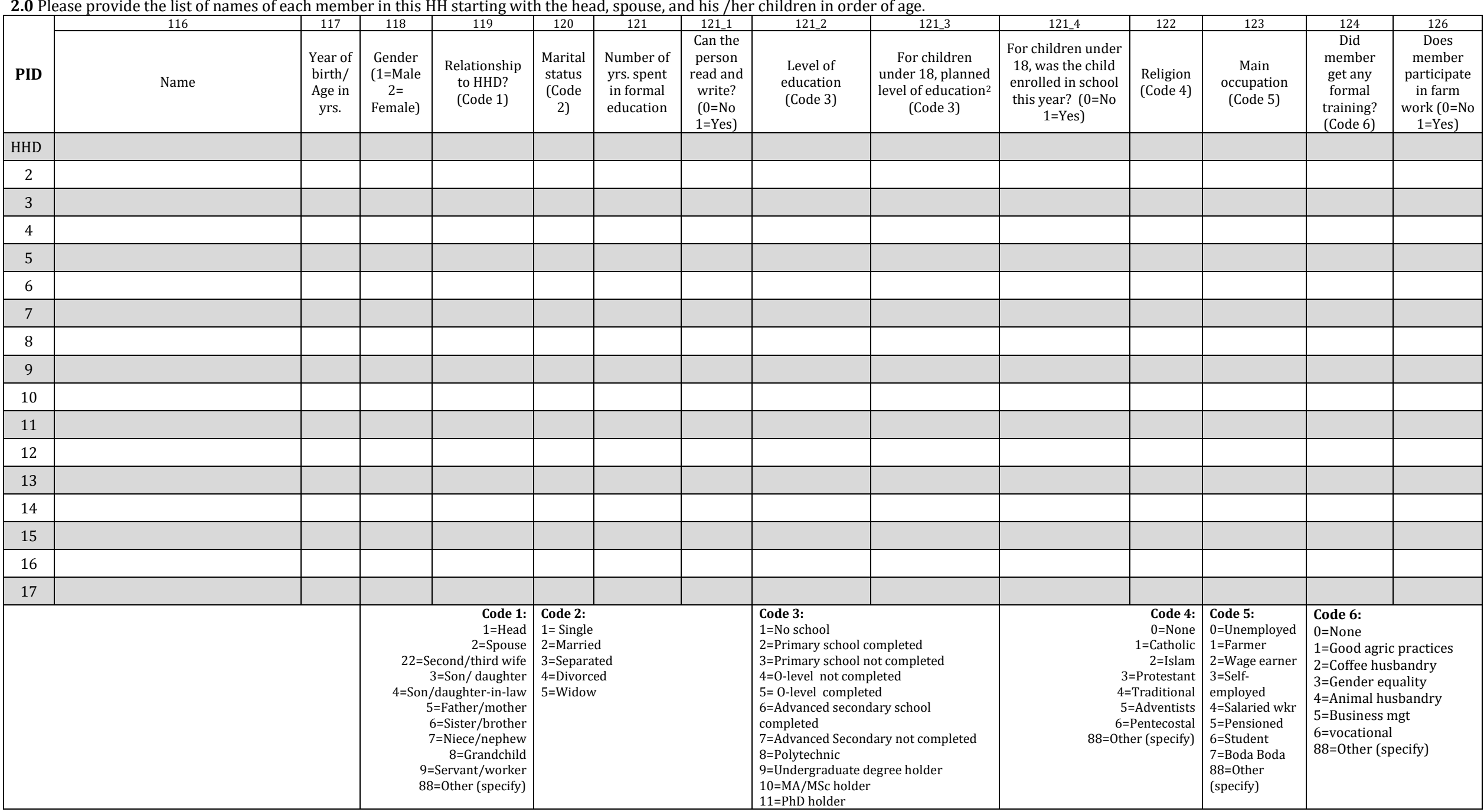

${ }^{1}$ Within the last 6 month, at least 3 days a week

2 Up to what level of education do the parents plan to educate the child? 
1.1 How long have you been a resident of this community?

$\mathbf{1 . 2}$ If new resident in this community, in which district were you a resident before settling in this community

1.3 Does this HH have any close relatives (parents, children, uncles, aunties, nieces, in-laws or nephews) leaving in cities away from this village?

1.4 How long have you been involved in farming? years

2.1 Are you a member of any farmer organization (includes association, cooperative, company)

2.2 Since when are you a member?
(indicate year)

$(0=\mathrm{No} \rightarrow$ move to $\mathrm{Q} 3 \quad 1=\mathrm{Yes})$

2.3 Who within the HH is a registered member? (1=HHD 2=Spouse $3=$ Both $88=0$ ther (specify))

3.1 Are you aware of any of the following certification schemes: Organic, Fairtrade, or UTZ?

3.2 Are you aware of any relative, neighbor or farmer that produces certified coffee?

3.3 If YES, do you interact with any of these persons?

$(0=$ No, $1=$ Yes $)$

$(0=\mathrm{No}, 1=\mathrm{Yes})$

4 Is or was this HH ever certified (that is under UTZ, Fairtrade, organic certified) to grow coffee? $(0=\mathrm{No}, 1=\mathrm{Yes})$

5.0 In which of the following certification scheme(s) (did) the HH participate?

\begin{tabular}{|c|l|l|}
\hline & & $\begin{array}{l}0=\text { No, never (move to next scheme) } \\
1=\text { Yes } \\
2=\text { Only formerly }\end{array}$ \\
\hline 1 & UTZ & \\
\hline 2 & Organic & \\
\hline 3 & Fairtrade & \\
\hline 5 & Area certified &
\end{tabular}

$(0=$ No, never $\rightarrow$ move to $Q 4 \quad 1=$ Yes $)$

6.0 Provide information on the total land area owned in the last 12 months.

\begin{tabular}{|c|c|c|c|c|}
\hline & 1 & 2 & 4 & 5 \\
\hline & & Current area (acres) & What type of title do you hold for this land? (Code 1) & How did you acquire this land? (Code 2) \\
\hline 1 & Total land owned & & & \\
\hline 2 & Land rented-in & & & \\
\hline 3 & Land rented-out & & & \\
\hline 4 & Total area cultivated & & & \\
\hline 5 & Area under pasture & & & \\
\hline 6 & Fallowed land & & & \\
\hline 7 & Area under coffee & & & \\
\hline
\end{tabular}

Code 1: 1=Freehold 2=Leasehold 3=Mailo 4=Customary rights 5=Bibanja

Code 2: $1=$ Purchased $\quad 2=$ Inherited/given by parents/relatives $3=$ Inherited (spouse) $\quad 4=$ Agreement with land/use rights owner $88=0$ ther (specify)

7.1 On average, how much time (in min) do you spend walking from your homestead to the NEAREST field? 
3.1 What is the average age [years] of your current productive coffee trees':

3.2 What is the average age [years] of your current unproductive coffee trees ${ }^{1}$

4 Is coffee production a tradition or a business for you? years.

5.0 Please provide information on your coffee production and marketing in the last 12 months.

\begin{tabular}{|c|c|c|c|c|c|c|c|c|}
\hline 36 & 37 & 38 & 39 & 40 & 41 & 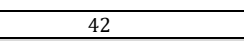 & 43 & 44 \\
\hline \multirow{2}{*}{$\begin{array}{l}\text { Total quantity of red cherries } \\
\text { harvested? (bag and kg per bag) }\end{array}$} & \multicolumn{4}{|c|}{ Quantity of coffee sold (kg) } & \multicolumn{4}{|c|}{ Coffee price received by farmer } \\
\hline & Flowers $^{2}(\mathrm{~kg})$ & $\operatorname{Red}^{3}(\mathrm{~kg})$ & Kiboko $^{4}(\mathrm{~kg})$ & $\operatorname{Kasse}^{5}(\mathrm{~kg})$ & Flowers $^{2}($ Shs $/ \mathrm{kg})$ & $\operatorname{Red}^{3}($ Shs/kg) & Kiboko $^{4}(\mathrm{Shs} / \mathrm{kg}$ ) & Kasse $^{5}($ Shs/kg) \\
\hline _______ bags & & & & & & & & \\
\hline One bag $=\ldots \ldots$ _ $\mathrm{kg}$ & & & & & & & & \\
\hline Total quantity: & kg & $\mathbf{k g}$ & kg & $\mathbf{k g}$ & Shs $/ \mathrm{kg}$ & Shs $/ \mathrm{kg}$ & Shs $/ \mathrm{kg}$ & Shs $/ \mathrm{kg}$ \\
\hline
\end{tabular}

6.0 Please provide the following information on coffee marketing:

\begin{tabular}{|c|c|c|c|c|c|c|c|c|c|}
\hline & & 84 & 85 & 86 & 87 & 88 & 89 & 90 & 91 \\
\hline & & \multicolumn{4}{|c|}{ Certified coffee } & \multicolumn{4}{|c|}{ NON-Certified coffee } \\
\hline & & Flower $^{2}$ & Red cherries $^{3}$ & Kiboko $^{4}$ & Kasse $^{5}$ & Flowers $^{2}$ & Red cherries $^{3}$ & Kiboko $^{4}$ & Kasse $^{5}$ \\
\hline 2 & $\begin{array}{l}\text { To whom do you normally sell the following type of coffee? (Allow multiple } \\
\text { responses BUT start with most important)? (Code 1) }\end{array}$ & & & & & & & & \\
\hline 3 & Who in the HH negotiates for coffee prices with buyers? (Code 2) & & & & & & & & \\
\hline
\end{tabular}

Code 1: 1=Farmer group 2=Middlemen 3=Kibinge FA 4=IBERO LTD 5=KAWACOM 6=Market in Kampala 7=Local exporter $8=$ =Farmer organization/association/company/cooperative $88=0$ ther (specify)

Code 2: $1=$ HHD $2=$ Spouse $3=$ Jointly $88=0$ ther (specify)

${ }_{1}$ Unproductive coffee is referred to as young coffee trees (gardens where coffee was only planted recently and does not yet produce coffee)

2 Flowers: Coffee sold in the field as flowers (also include Green berries)

${ }^{3}$ Red cherries: Wet coffee still in husks sold soon after harvesting

5 Kasse (or FAQ (Fair Average Quality): Dry, hulled green beans but not graded 
SECTION 4: LAND AREA AND GENERAL CROPS GROWN (A1)

1.0 Please provide the following details on ALL crops grown by the HH in the last 12 months. (Capture details for all the crops grown for the main harvest season and fly-harvest season'1).

\begin{tabular}{|c|c|c|c|c|c|c|c|c|c|c|c|c|c|c|c|}
\hline & & & & & & & & & Usuall & who in th & $\overline{\text { e HH (C }}$ & de 4).... & & & \\
\hline 10 & $10 \_1$ & 11 & $11 \_1$ & 12 & 13 & 14 & & $14 \_1$ & $14 \_2$ & 15 & 15 & 16 & $16 \_1$ & 17 & | Code 1: \\
\hline & $\begin{array}{l}\text { Season } \\
\text { grown } 1\end{array}$ & & $\begin{array}{l}\text { Inter- } \\
\text { cropped }\end{array}$ & & $\begin{array}{l}\text { Total quantity } \\
\text { sold }\end{array}$ & Price $/ \mathrm{kg}$ recei & ved & $\begin{array}{l}\text { How } \\
\text { important }\end{array}$ & $\begin{array}{l}\text { is } \\
\text { respon- }\end{array}$ & $\begin{array}{l}\text { controls } \\
\text { decisions on }\end{array}$ & $\begin{array}{l}\text { harvests } \\
\text { it? }\end{array}$ & $\begin{array}{l}\text { controls } \\
\text { retained }\end{array}$ & $\begin{array}{l}\text { sells the } \\
\text { marketed }\end{array}$ & \begin{tabular}{|l|} 
controls \\
revenues?
\end{tabular} & $1=$ Coffee \\
\hline $\begin{array}{l}\text { (Code } \\
\text { 1) }\end{array}$ & $\begin{array}{c}2=\text { Fly } \\
3=\text { Both) }\end{array}$ & $\begin{array}{l}\text { area } \\
\text { grown } \\
\text { (acre) }\end{array}$ & $\begin{array}{l}(0=\text { No } \\
1=\text { Yes })\end{array}$ & $\begin{array}{c}\text { harvested } \\
\text { (units*conversion } \\
\text { factor }(\mathrm{kg}) \text { ) }\end{array}$ & $\begin{array}{l}\text { Total quantity } \\
\text { (kg) }\end{array}$ & $\begin{array}{r}\text { Average } \\
\text { price per } \\
\text { unit }\end{array}$ & $\begin{array}{r}\text { Unit } \\
\text { code } \\
\text { (Code } \\
2)]\end{array}$ & $\begin{array}{l}\text { these } \\
\text { crops for } \\
\text { your HH? } \\
\text { (Code 3) }\end{array}$ & $\begin{array}{l}\text { this } \\
\text { crop? }\end{array}$ & activities? & & narvest? & proportuon? & & $\begin{array}{l}\text { 3=Pineapples } \\
\text { 4=Maize } \\
\text { 5=Beans } \\
\text { 6=Sweet } \\
\text { potatoes }\end{array}$ \\
\hline Coffee & & & & & & & & & & & & & & & $\begin{array}{l}\text { 7=Sweet } \\
\text { bananas } \\
\text { 8=Irish potatoes } \\
\text { 9=Groundnuts }\end{array}$ \\
\hline & & & & $-^{*}$ & - & & & & & & & & & & $\begin{array}{l}10=\text { Cowpeas } \\
11=\text { Tomatoes }\end{array}$ \\
\hline & & & & Units $\mathrm{kg} / \mathrm{unit}$ & Units $\mathrm{kg} / \mathrm{unit}$ & Shs & & & & & & & & & 12=Rice \\
\hline & & & & $--1+2>$ & & & & & & & & & & & $\begin{array}{l}14=\text { Sorghum } \\
15=\text { Onion }\end{array}$ \\
\hline & & & & Units $\mathrm{kg} / \mathrm{unit}$ & Units $\mathrm{kg} / \mathrm{unit}$ & Shs & & & & & & & & & $17=$ Sukuma \\
\hline & & & & - & $*$ & & & & & & & & & & $\begin{array}{l}18=\text { Sugarcane } \\
19=\text { Soybean }\end{array}$ \\
\hline & & & & Units $\mathrm{kg} / \mathrm{unit}$ & Units $\mathrm{kg} / \mathrm{unit}$ & Shs & & & & & & & & & 20=Pumpkin \\
\hline & & & & - & $*$ & & & & & & & & & & $\begin{array}{l}\text { fruits } \\
22=\text { Cabbages }\end{array}$ \\
\hline & & & & Units $\mathrm{kg} /$ unit & Units $\mathrm{kg} / \mathrm{unit}$ & Shs & & & & & & & & & $23=$ Leafy \\
\hline & & & & - & & & & & & & & & & & 88=0ther \\
\hline & & & & Units $\mathrm{kg} / \mathrm{unit}$ & Units $\mathrm{kg} / \mathrm{unit}$ & Shs & & & & & & & & & (specify) \\
\hline & & & & & ${ }^{*}$ & & & & & & & & & & $\begin{array}{l}\text { Code 2: } \\
1=\mathrm{kg}\end{array}$ \\
\hline & & & & Units $\mathrm{kg} / \mathrm{unit}$ & Units $\mathrm{kg}$ /unit & Shs & & & & & & & & & $2=\mathrm{Bag}$ \\
\hline & & & & $=-1+2>$ & & & & & & & & & & & $\begin{array}{l}3=\text { =Bunch } \\
4=\text { Basket }\end{array}$ \\
\hline & & & & Units $\mathrm{kg} / \mathrm{unit}$ & Units $\mathrm{kg} / \mathrm{unit}$ & Shs & & & & & & & & & $5=$ Debe \\
\hline & & & & - $^{*}$ & ${ }^{*}-$ & & & & & & & & & & \\
\hline & & & & Units $\mathrm{kg} /$ unit & Units $\mathrm{kg} / \mathrm{unit}$ & Shs & & & & & & & & & \\
\hline
\end{tabular}

Code 3: 1=Very important 2=Important 3=Not so important



${ }^{1}$ For Masaka district: Main season June-July and Fly season Dec-Jan; for Luwero district: Main season Dec -March and Fly in Aug-Oct

2 Importance in terms of revenues and own consumption 
SECTION 5: PRODUCTION COSTS FOR GENERAL CROPS AND COFFEE (A2/B2)

1.0 For your coffee gardens (total area), provide details on the costs of production in the past 12 months (i.e. for the two seasons ${ }^{1}$ ). Then ask for additional costs accuring for other crops individually, especially seeds and pesticides. Make sure to capture all costs (all categories) if the respective crop is not intercropped with coffee.

\begin{tabular}{|c|c|c|c|c|c|c|c|c|c|c|c|c|c|c|c|c|}
\hline \multirow{4}{*}{\begin{tabular}{|c|}
18 \\
\\
(use \\
Code 1)
\end{tabular}} & \multirow{4}{*}{$\begin{array}{l}\text { Total area } \\
\text { (acre) }\end{array}$} & & & & & & & & & & & \multirow{4}{*}{$\begin{array}{l}52 \\
\text { Hired } \\
\text { labor } \\
\text { Total } \\
\text { cost } \\
\text { (Shs) }\end{array}$} & & \multirow{4}{*}{$\begin{array}{c}53 \\
\text { Machiner } \\
\text { y total } \\
\text { cost } \\
(\mathrm{Shs})^{2}\end{array}$} & \multirow{4}{*}{$\begin{array}{c}54 \\
\text { Other } \\
\text { costs }^{3} \\
\text { (Shs) }\end{array}$} & \multirow{13}{*}{$\begin{array}{l}\text { Code 1: } \\
\text { 1=Coffee } \\
\text { 2=Matoke } \\
\text { 3=Pineapples } \\
\text { 4=Maize } \\
\text { 5=Beans } \\
\text { 6=Sweet } \\
\text { potatoes } \\
\text { 7=Sweet } \\
\text { bananas } \\
\text { 8=Irish potatoes } \\
\text { 9=Groundnuts } \\
\text { 10=Cowpeas } \\
\text { 11=Tomatoes } \\
\text { 12=Rice } \\
\text { 13=Cassava } \\
\text { 14=Sorghum } \\
\text { 15=Onion } \\
\text { 17=Sukuma } \\
\text { 18=Sugarcane } \\
\text { 19=Soybean } \\
\text { 20=Pumpkin } \\
\text { 21=Passion } \\
\text { fruits } \\
\text { 22=Cabbages } \\
\text { 23=Leafy } \\
\text { vegetables } \\
\text { 88=Other } \\
\text { (specify) }\end{array}$} \\
\hline & & 19 & \begin{tabular}{|l|}
20 \\
\end{tabular} & \begin{tabular}{l|l}
46 & \\
\end{tabular} & 47 & 48 & 49 & $49 \_1$ & 50 & 51 & $51 \_1$ & & $\begin{array}{c}52 \_1 \\
\end{array}$ & & & \\
\hline & & \multicolumn{2}{|c|}{ Seeds/seedlings } & \multicolumn{2}{|c|}{ Synthetic fertilizer } & \multicolumn{3}{|c|}{ Manure } & \multicolumn{3}{|c|}{ Pesticides, herbicides, fungicides } & & Tarpaulins & & & \\
\hline & & $\begin{array}{c}\text { Total } \\
\text { quantity } \\
\text { (kg or unit) }\end{array}$ & $\begin{array}{c}\text { Cost } \\
\text { (Shs/kg or } \\
\text { unit) }\end{array}$ & $\begin{array}{c}\text { Total } \\
\text { quantity } \\
(\mathrm{kg})\end{array}$ & $\begin{array}{l}\text { Cost } \\
\text { (Shs/ } \\
\text { kg) }\end{array}$ & $\begin{array}{l}\text { Quantity } \\
\text { (kg) }\end{array}$ & $\begin{array}{c}\text { Cost } \\
\text { (Shs/kg) }\end{array}$ & $\begin{array}{l}\text { Type } \\
\text { (Code } \\
2 \text { ) }\end{array}$ & $\begin{array}{c}\text { Total } \\
\text { quantity (kg } \\
\text { or l) }\end{array}$ & $\begin{array}{c}\text { Cost } \\
\text { (Shs/unit) }\end{array}$ & $\begin{array}{c}\text { Type } \\
\text { (Code } \\
3 \text { ) }\end{array}$ & & $\begin{array}{l}\text { Total cost } \\
\text { (Shs) }\end{array}$ & & & \\
\hline $\begin{array}{l}\text { Coffee } \\
\text { (coffee } \\
\text { garden) }\end{array}$ & & & & & & & & & & & & & & & & \\
\hline & & & & & & & & & & & & & & & & \\
\hline & & & & & & & & & & & & & & & & \\
\hline & & & & & & & & & & & & & & & & \\
\hline & & & & & & & & & & & & & & & & \\
\hline & & & & & & & & & & & & & & & & \\
\hline & & & & & & & & & & & & & & & & \\
\hline & & & & & & & & & & & & & & & & \\
\hline & & & & & & & & & & & & & & & & \\
\hline
\end{tabular}

Code 2: $1=$ Cow dung $2=$ Cooking material refuse $3=$ Compost $4=0$ ther (specify)

Code 3: $1=$ =Round-up, $2=$ Gramaxon, $3=$ Dudu bitooke, $4=$ Weedmaster $5=$ Antkiller $6=0$ thers (specify)

${ }^{1}$ For Masaka district: Main season June-July and Fly season Dec-Jan; for Luwero district: Main season Dec -March and Fly in Aug-Oct

If
If the farmer owns machinery ask for the cost of operation (e.g. fuel, hiring, maintenance). We are only interested in the variable costs.

3 Other additional costs incurred by the farmer (e.g. packaging). 


\section{SECTION 6: FARMING PRACTICES AND POST-HARVEST MANAGEMENT IN COFFEE}

\begin{tabular}{|c|l|}
\hline 1 & Do you intercrop or use crop cover on your coffee fields? \\
\hline 2 & Do you employ erosion measures (e.g. terraces) in your coffee gardens? \\
\hline 3 & On average, how many shade trees do you have in your gardens? (trees/acre) \\
\hline 4 & Did you cut any shade trees within the last 12 months? \\
\hline 5 & Do you use mulching/composting? \\
\hline 6 & Do you use organic pesticides? \\
\hline 7 & $\begin{array}{l}\text { Do you/ would you find it difficult to access chemical pesticides, herbizides, } \\
\text { fungicides? (e.g. because the products are too expensive, store too far away, etc.) }\end{array}$ \\
\hline 8 & Do you prune your coffee? \\
\hline 9 & Do you sometimes pick yellow or green cherries to reduce time spend on picking? \\
\hline 10 & Do you sometimes pick green cherries when you face urgent cash needs? \\
\hline 11 & How do you dry your coffee? [indicate most important one] \\
\hline 12 & How do you store your coffee after drying it? [indicate most important one] \\
\hline 13 & How do you ascertain the moisture content? [indicate most important one] \\
\hline 14 & What would you say is the factor constraining your coffee production most? \\
\hline 15 & Do you keep records for your coffee production activities? [if no, move to next section] \\
\hline $15 a$ & \\
\hline 15 & \\
\hline 16 & Who is usually responsible for record keeping? [indicate most important] \\
\hline 17 & Is someone helping that person in record keeping? [indicate most important] \\
\hline & \\
\hline
\end{tabular}

\begin{tabular}{|c|c|}
\hline & \\
\hline & \\
\hline & \\
\hline & \\
\hline & \\
\hline & \\
\hline & \\
\hline & \\
\hline & \\
\hline & \\
\hline & 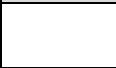 \\
\hline & \\
\hline & \\
\hline & \\
\hline & \\
\hline & \\
\hline & \\
\hline
\end{tabular}

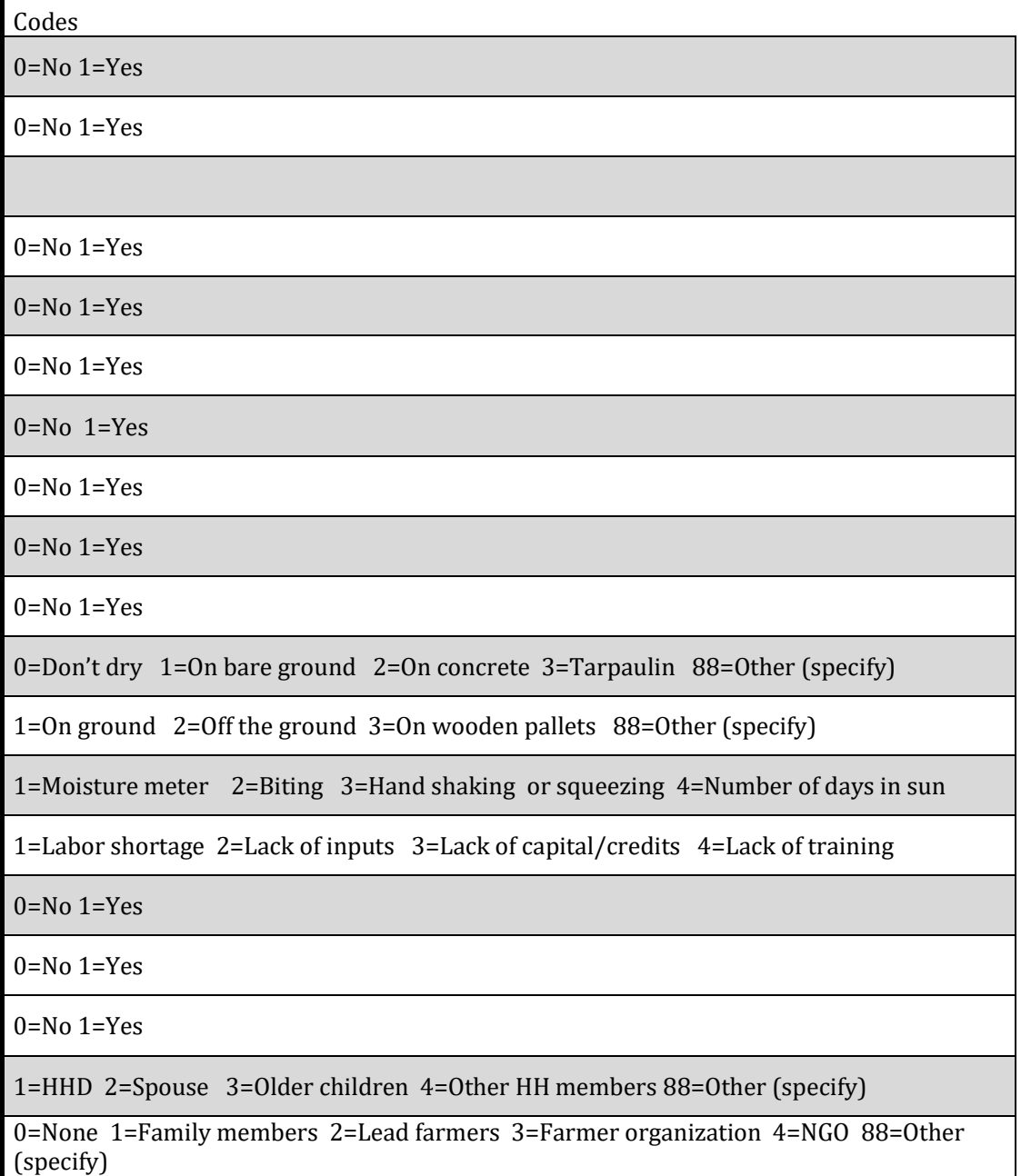


SECTION 7: LIVESTOCK PRODUCTION (C)

1.0 Please provide the following information on livestock owned by your $\mathrm{HH}$ in the past $\mathbf{1 2}$ months.

\begin{tabular}{|c|c|c|} 
Livestock and & 100 & $100 \_2$ \\
\cline { 2 - 3 } & 10mber & Who1
\end{tabular}

\begin{tabular}{l|c|c}
$\begin{array}{c}\text { livestock } \\
\text { products }\end{array}$ & $\begin{array}{c}\text { Number } \\
\text { currently }\end{array}$ & $\begin{array}{c}\text { Who }^{1} \\
\text { owns it? }\end{array}$ \\
\hline
\end{tabular} 101
Estimated total current
value if all items sold Did you sell [item] in
the last 12 months
$(0=$ No $1=$ Yes $)$

\begin{tabular}{|l|l|c|}
\hline \multirow{2}{*}{$\begin{array}{c}\text { If yes, what was the } \\
\text { total value received } \\
\text { (Shs) }\end{array}$} & \multicolumn{3}{|c|}{105} & \multicolumn{1}{|c|}{ Total Cost of Prod } \\
\cline { 2 - 3 } & Fodder & Hired labor \\
\hline & & \\
\hline & & \\
\hline & & \\
& & \\
& &
\end{tabular}

\begin{tabular}{|c|c|}
\hline 107 & $\mid$ \\
\hline & Veterinary \\
\hline & \\
\hline & \\
\hline
\end{tabular}

Other costs

Code 1:

(Code 1) \begin{tabular}{|rr} 
(Code 2) & (Shs) \\
& Shs \\
& Shs \\
& Shs \\
& Shs \\
\hline & Shs \\
& Shs \\
& Shs
\end{tabular}

Code 2: $1=$ HHD 2=Spouse $3=$ Jointly $88=0$ ther (specify)

\section{SECTION 8: ASSET OWNERSHIP (D)}

1.0 Please provide the following information on assets owned by this HHD in the past 12 months?

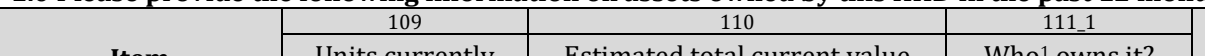

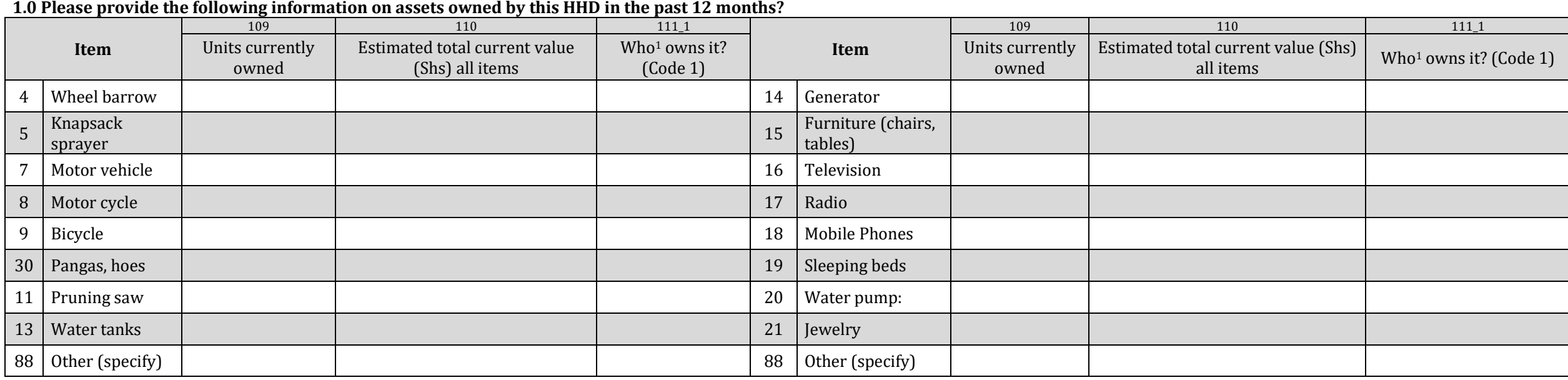

Code 1: $1=$ HHD 2=Spouse 3=Jointly 4=N.A. $88=0$ ther

${ }^{1}$ If more than one item, add ID Code

2Value of all livestock and livestock products sold by the household in the past 12 months 
2.0 Provide the following information on $\mathrm{HH}$ assets currently owned.

\begin{tabular}{|c|c|c|c|}
\hline \multicolumn{2}{|c|}{ 然 } & \multirow{2}{*}{ Current ownership } & \\
\hline 1 & Type of dwelling & & $\begin{array}{l}\begin{array}{l}1=\text { =Mud hut with grass thatching } 2=\text { =Mud hut with asbestos/iron roof } 3=\text { =Brick house with grass thatching } 4=\text { =Brick house with } \\
\text { asbestos/iron roof }\end{array} \\
\end{array}$ \\
\hline 2 & Tenure status of dwelling & & $1=0$ wn with title deeds $2=0$ wn without title deeds $3=$ Rented $\quad 4=$ Borrowed without pay $88=0$ ther (specify) \\
\hline 3 & Total number of rooms owned & & \\
\hline 4 & Type of toilet & & $1=$ Bush $\quad 2=$ Flush $3=$ Ventilated latrine $\quad 4=$ Pit latrine $\quad 88=0$ ther (specify) \\
\hline 5 & Main source of drinking water & & $1=$ Private tap $2=$ =Public tap/borehole 4=River, stream, lake, pond, well, springs $\quad 5=$ Rain water $\quad 88=0$ ther (specify) \\
\hline 6 & Do you usually treat your drinking water? & & 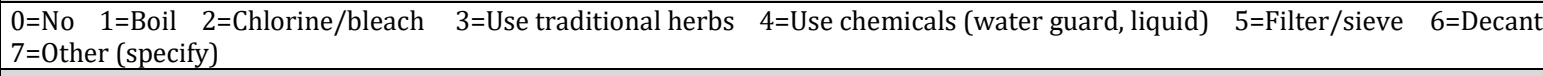 \\
\hline 7 & Main source of lighting & & 1=Electric bulbs $\quad 2=$ =Paraffin lantern $3=$ =andles $\quad 4=$ Wick Lamp $88=0$ ther (specify) \\
\hline 8 & Main type of cooking fuel & & 1=Charcoal $2=$ Firewood $3=$ =as $\quad 4=$ =Electricity $\quad 5=$ =Paraffin/Kerosene $\quad 6=$ Solar $\quad 7=$ Biogas $\quad 88=0$ ther (specify) \\
\hline
\end{tabular}

2.9 Does this HH have any close wealthy ancestors (parents, uncles, aunties, nieces, in-laws or nephews)? 1=Yes, 0=No

3.0 Provide information on how much Off-farm income was earned by members of this HH in the past 12 months. (Please use PID)

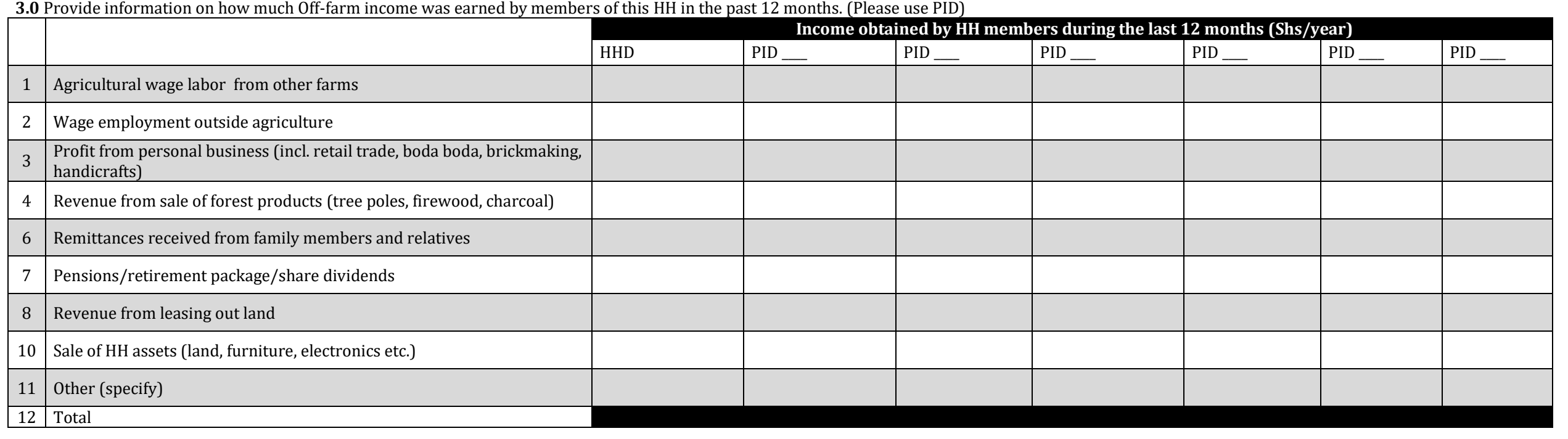


SECTION 9: NON-FOOD EXPENDITURE (H)

1.0 Consider the last 12 months, generally how much has your HH spent on the items listed in a typical month, term, or year (see specification indicated for each item)? (Enter zero if nothing is consumed. Enter 99 if they don't know)

\begin{tabular}{|c|c|c|c|c|c|}
\hline \multicolumn{2}{|r|}{ Item } & \multirow{2}{*}{$\begin{array}{c}162 \\
\begin{array}{c}\text { Expenditure (total value in } \\
\text { Shs) }\end{array} \\
\end{array}$} & \multicolumn{2}{|r|}{ Item } & \multirow{2}{*}{$\begin{array}{c}163 \\
\text { Expenditure (total value in Shs) }\end{array}$} \\
\hline 1 & Rentals (house) per month & & 8 & Telephone bills (including mobile) per month & \\
\hline $1 \mathrm{~b}$ & Rentals land per year & & 9 & Ceremonies (church, weddings, festivals, burials) per year & \\
\hline 2 & Kitchen utensils (pots, pans, plates, cutlery) per year & & 10 & Firewood/Charcoal per month & \\
\hline 3 & HH furniture (beds, tables, chairs) per year & & 11 & Remittances or transfers to other individuals per year & \\
\hline 4 & Clothing and footwear (fabric, clothes, towels, shoes) per year & & 12 & Repairs of machinery, equipment, housing per year & \\
\hline 5 & Electricity, Gas, Paraffin, Biogas (for cooking and light) per month & & 13 & Amount paid as interest, other fees, amagoba per month & \\
\hline 6 & Education, books, school fees, uniforms per term & & 14 & Public transport (boda-boda, taxi, bus) per month & \\
\hline 7 & Health care (consultation fees, medicines, spectacles) per year & & 30 & Hygiene and beauty products, cosmetics per year & \\
\hline 15 & Membership fees (COOP, Fmr groups, Burial societies per year & & 88 & Other major non-food items (specify) per month & \\
\hline
\end{tabular}

2.0 In the past 12 months, how much did your HH spend on the items for individual HH members under 25? (Use PID; write down "0" if the item/service is provided for free and "88" if not applicable)

\begin{tabular}{|c|c|c|c|c|c|}
\hline $\begin{array}{l}\text { HH member } \\
\text { PID }\end{array}$ & $\begin{array}{l}\text { How much do you pay for school } \\
\text { fees and uniforms for [NAME]? }\end{array}$ & $\begin{array}{l}\text { For extra classes, books, and stationary } \\
\text { (exercise books, pens, etc.)?? }\end{array}$ & $\begin{array}{l}\text { For transport to } \\
\text { school? }\end{array}$ & $\begin{array}{l}\text { For health related costs (vaccinations, medical } \\
\text { consultation, medicine, other health measures) }\end{array}$ & For clothing and footwear? \\
\hline & & & & & \\
\hline & & & & & \\
\hline & & & & & \\
\hline & & & & & \\
\hline & & & & & \\
\hline & & & & & \\
\hline & & & & & \\
\hline & & & & & \\
\hline
\end{tabular}

SECTION 10: FOOD EXPENDITURE (GI)

$10 \mathrm{n}$ average, how many people were present in the last 7 days? In this section children are defined as persons under 18 years.

\begin{tabular}{|c|c|c|c|}
\hline \multicolumn{3}{|c|}{ 1On average, how many people were present in the last 7 days? In this section children are defined as } \\
\hline \multicolumn{3}{|c|}{ HH members } \\
\hline \multicolumn{3}{|c|}{ Adults } & \multicolumn{2}{c|}{ Children (under 18) } \\
\hline Male & Female & Male & Female \\
\hline & & & \\
\hline & & & \\
\hline
\end{tabular}

\begin{tabular}{|c|c|c|c|c|}
\hline \multicolumn{3}{|c|}{ Visiting members } \\
\multicolumn{3}{|c|}{ Adults } & & \multicolumn{2}{c|}{ Children (under 18) } \\
\hline Male & Female & Male & Female \\
\hline & & & & \\
\hline
\end{tabular}


2.0 In the past 7 days indicate how much of the following food items your HH consumed and the value in Shs. (For all food consumed, including own-produced, bought, gifts and from food aid program, by all people living in $\mathrm{HH}$ )

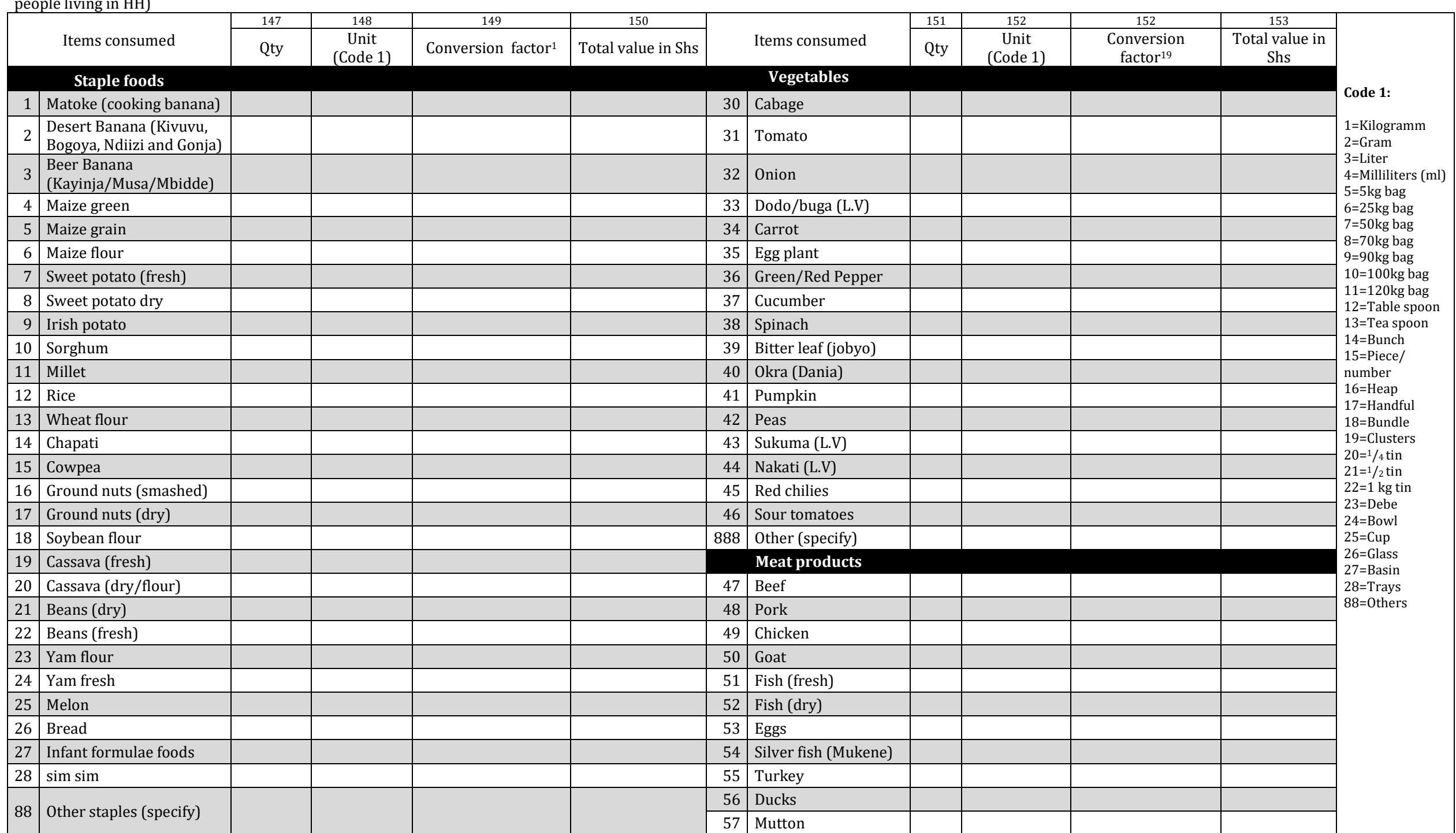

${ }^{1}$ For items that are not clearly transferrable (bundle, bunch, etc.), indicate corresponding amount of kg/liter) 
2.0 Continued (In the past 7 days indicate how much of the following food items your HH consumed and the value in Shs. (For all food consumed, including own-produced, bought, gifts and from food aid program, by all people living in $\mathrm{HH}$ ))

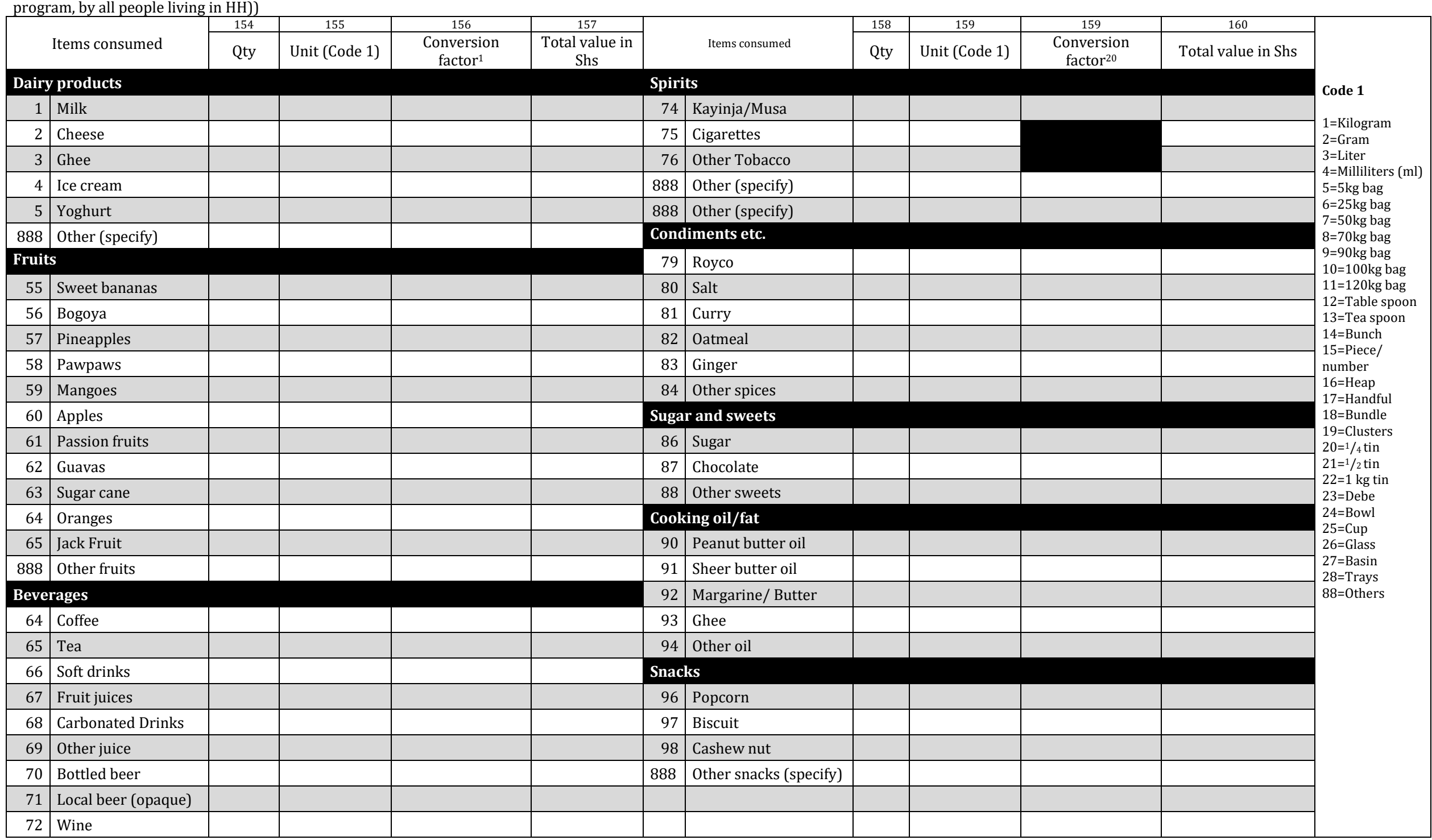

${ }^{1}$ For items that are not clearly transferrable (bundle, bunch, etc.), indicate corresponding amount of $\mathrm{kg} / \mathrm{liter}$ 
3.1 During the past 7 days, did any of the HH members eat food away from home (e.g. in schools, restaurants, during ceremonies)?____ (0=No 1=Yes)

3.2 If yes, indicate the number of times they ate food away and the value of meals in the past 7days.

\begin{tabular}{|c|c|c|c|c|c|c|c|c|c|c|c|}
\hline & & HHD & PID:___ & PID: & PID:__ & _ & PID:___ & PID: & PID:_ & PID: & PID:_ \\
\hline 1 & Number of times & & & & & & & & & & \\
\hline 2 & $\begin{array}{l}\text { Value of meals (Shs) (total for all } 7 \\
\text { days) }\end{array}$ & & & & & & & & & & \\
\hline 3 & $\begin{array}{l}\text { Which foods does member normally } \\
\text { eat (Code 1) }\end{array}$ & & & & & & & & & & \\
\hline
\end{tabular}

Code 1: 1=Beans 2=Posho 3=Maize porridge 4=Meat 5=Fish 6=Rice 88=0ther (specify)

\section{SECTION 11: SERVICES (I)}

1.0 How far in kilometers would you have to travel to access the following services? (Indicate distance to nearest source in $\mathrm{km}$ )

\begin{tabular}{|c|c|c|c|c|c|}
\hline & & Distance to nearest source $(\mathrm{km})$ & & & Distance to nearest source $(\mathrm{km})$ \\
\hline 1 & Financial credit & & 12 & Tarmac road (and bus connection to Kampala) & \\
\hline 22 & Micro-credit and saving facility & & 15 & Electricity & \\
\hline 10 & Commercial bank & & 16 & Water source for $\mathrm{HH}$ use & \\
\hline 3 & Input market for coffee & & 14 & Watering source (livestock) & \\
\hline 4 & Output market for coffee (different from farm-gate) & & 18 & Internet & \\
\hline 5 & Coffee collection center & & 20 & Religious facilities & \\
\hline 6 & Extension office/r & & 22 & Hospital, clinic or doctor & \\
\hline 21 & Coffe nurseries run by fellow farmers & & 7 & Primary school & \\
\hline 9 & Source of coffee seedlings & & 8 & Secondary school & \\
\hline 11 & Promoter lead farmer for coffee & & 19 & University & \\
\hline
\end{tabular}




\section{SECTION 12: MOBILE PHONE USE WITHIN HOUSEHOLDS}

1.1 Did anyone in this HHD own a mobile phone handset?

$(0=$ No $1=$ Yes $) \rightarrow$ If NO, move to the next section!

1.2 Which year did the earliest user of a mobile phone in this HH start using a mobile phone? [indicate year]

2.0

\begin{tabular}{|c|c|c|c|c|c|c|c|}
\hline & & Currently (2015) & in 2012 & & & \begin{tabular}{|l} 
Currently \\
\end{tabular} & in 2012 \\
\hline 1 & $\begin{array}{l}\text { Is /Was your HH's area covered with mobile phone network? } \\
(\mathrm{No}=0 \text { Yes=1) }\end{array}$ & & & 5 & $\begin{array}{l}\text { How far was the (nearest) shop/place where members of this HH could } \\
\text { buy/bought a mobile phone handset? }\end{array}$ & $\mathrm{km}$ & $\mathrm{km}$ \\
\hline 2 & $\begin{array}{l}\text { Is/Did this HH's area have mobile phone telecom service center } \\
\text { in nearest town? ( } \mathrm{No}=0 \text { Yes }=1)\end{array}$ & & & 6 & $\begin{array}{l}\text { How far was the (nearest) shop/place where members of this HH } \\
\text { could/bought airtime? }\end{array}$ & $\mathrm{km}$ & $\mathrm{km}$ \\
\hline 3 & $\begin{array}{l}\text { Did sometimes members of this HH use neighbors' or friends' } \\
\text { phones for calls or messages? [ALL] (No=0 Yes }=1 \text { ) }\end{array}$ & & & 7 & $\begin{array}{l}\text { How far was the (nearest) shop/place where members of this HH } \\
\text { could/recharged the phone battery? }\end{array}$ & $\mathrm{km}$ & $\mathrm{km}$ \\
\hline 4 & $\begin{array}{l}\text { Of the TEN neighbors to this HH, how many of them have } \\
\text { mobile phones? (indicate number) }\end{array}$ & & & 8 & $\begin{array}{l}\text { Are/were there mobile phone or computer use training centers in your } \\
\text { HH's vicinity? }\end{array}$ & & \\
\hline
\end{tabular}

3.0

Who in this HH owned a mobile phone? (For only those who had, record their PIDs under respective

1 years in second and third column on the right).

Generally, how many times did (PERSON (record PID in second and third column under respective

year)) use mobile phone per day? (Making and receiving calls)?

3 How many network bars (reflecting network strength) is/would your mobile phone show?

4 Which Mobile Phone network provider is $\backslash$ was the HHD subscribed to?

(1=MTN 2=Airtel 3=UTL 4=KT 5=Orange 6=Smile 7=Warid 8=Vodafone 9=0thers (specify))

How does $\backslash$ did the HHD rate the quality of telephone network coverage here?

(1= very good $2=$ Good $3=$ Fair $4=$ =Poor $5=$ =very poor $)$

6 On average, how much did the HHD spend in total on airtime per month?

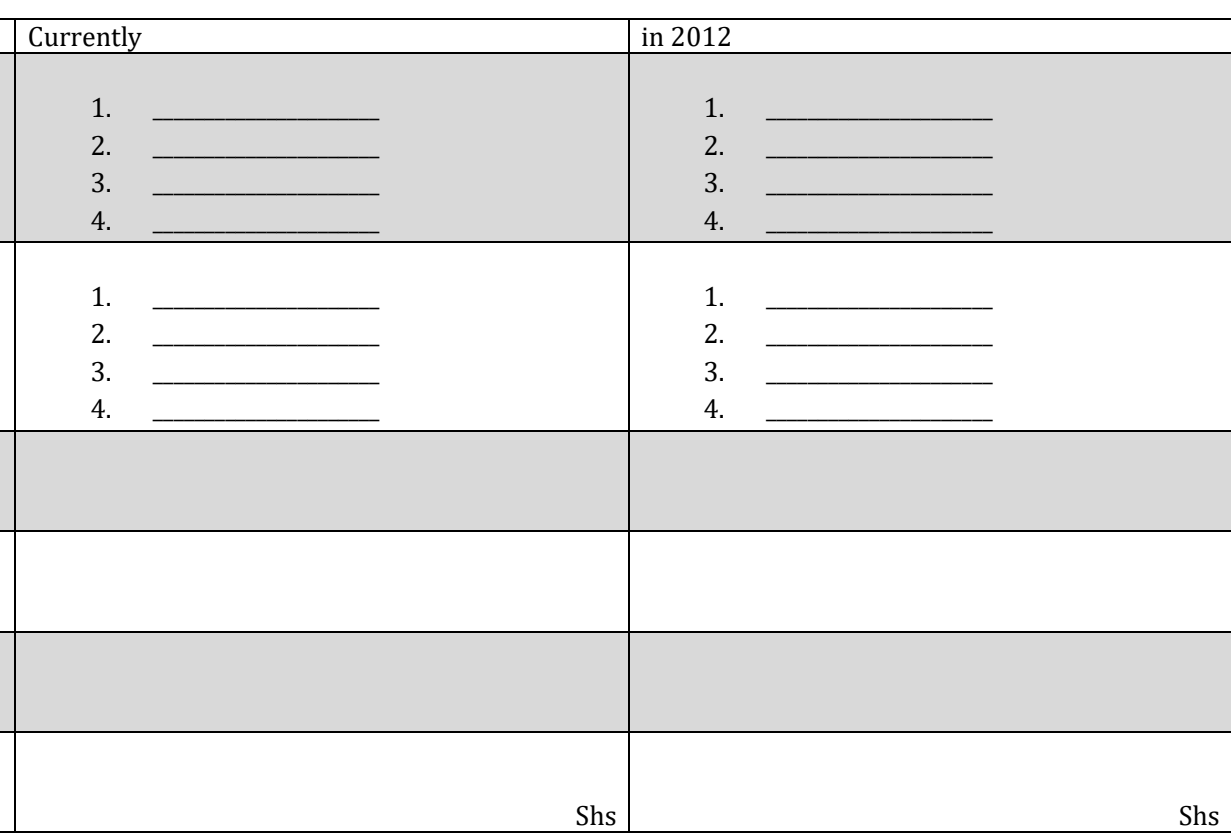


1 Does any member in this HH use mobile money services?

$(0=$ No $1=$ Yes $) \rightarrow$ If NO, move to the next section!

2 Which year did the earliest user of MM in this $\mathrm{HH}$ start using mobile money services? Year

\begin{tabular}{|c|c|c|c|}
\hline & & Currently & in 2012 \\
\hline 3 & Is/Was your HH's area covered with MM service network? & & \\
\hline 4 & Of TEN of this HH's nearest neighbors, how many in this village use MM? & & \\
\hline 5 & Did this HH's area have a MM service center in the nearest town? $(1=\mathrm{Yes}, 0=\mathrm{No})$ & & \\
\hline 6 & How far was the nearest MM service center where members of this HH could withdraw or deposit money into M-account? & $\mathrm{Km}$ & $\mathrm{Km}$ \\
\hline 7 & How much would it cost (one way trip) for a member from this HH to reach that MM center? & Shs & Shs \\
\hline 8 & Did any member of this HH, ever used friends' or neighbors MM services? (No=0 Yes=1) & & \\
\hline $8 \mathrm{~b}$ & If you received a text message could you open and read it? & & \\
\hline
\end{tabular}

$8 \mathrm{~b}$ If you received a text message could you open and read it?

9 Who in this HH used Mobile Money? [Record only PIDs for those who used MM in the second and third columns on the righ].

\begin{tabular}{|l|r|}
\hline Currently & in 2012 \\
\hline 1. & 1. \\
2. & 2. \\
\hline 3. & 3. \\
$4 .-$ & 4. \\
\hline & \\
\hline & \\
\hline & \\
\hline
\end{tabular}

10 On average, how much money did/would the HHD receive per year?

11 On average, how much money did/would the HHD send per year?

12 Which mobile money operator is the HHD subscribed to?

1=MTN 2=Airtel 3=UTL 4=Smile 5=0range 6=Easy Money 7=Vodafone 8=K2 9=Warid 10=0thers (specify)

13 Does/did the HHD know how much he/she is/was charged to send 10,000 UGX via MM services? (No=0 Yes=1)

14 What activity did the HHD mostly do via MM services? (tick ONE) (1=Withdrawing money 2=Sending Money 3=Paying bills 4=Paying school fees $5=$ =Saving money

14 6=Buying airtime $7=$ =Buying inputs $8=$ =Paying laborers $9=$ Transfer money $10=0$ thers (specify))

15 Generally, for WHAT purpose did the HHD receive most remittances via MM services? (tick ONE)

15 (1=Paying school fees $\quad 2$ =Given birth $3=$ =Had food shortage $\quad 4$ =Lost (dead) relative $\quad 5=$ =Social group function $\quad 6=$ General HH financial aid $\quad 7=0$ thers (specify))

16 To whom did the HHD mostly send money/remittances via MM services? Tick ONE (Code 6)

17 From whom did the HHD mostly receive money/remittances via MM services? Tick ONE (Code 6)

18 Averagely, how distant (in Km) was the person the HHD mostly sent/received money?

19 Did the HHD ever receive/pay back any money from the person he/she mostly sent/received money via MM services?

20 Did the HHD sometimes channel MM remittances sent/planned/aimed for a particular purpose to other abruptly urgent HH needs? (No=0, Yes=1)

Code 6: 1=Spouse 2=Son(s) 3=Daughter(s) 4=Friend(s) 5=Farmer group members 6=Father 7=Mother 8=Politician 9=Farm laborers 10=Business partners 11=0thers (specify)

22 Do you sometimes have severe HH misunderstandings? 1=Yes, 0=No 
Explain selection and measurement procedure to respondents. Select one male and one female infant (2-5 years) for the anthropometric measurement. Use random selection procedure if there are more than two potential infants. Also select the parents of the selected infants. If one or all of them are not present -or if the mother of the selected children is pregnant - use random selection procedure to select another male and/or female HH member.

1.0 Report information on each
\begin{tabular}{|l|l|}
\hline 1 & Name \\
\hline 2 & Person ID \\
\hline 3 & Age \\
\hline 4 & Name of the mother \\
\hline 5 & Name of the father \\
\hline 6 & $\begin{array}{l}\text { Is the selected female adult } \\
\text { pregnant? (If yes, do not } \\
\text { continue. Select another } \\
\text { female HH member!) }\end{array}$ \\
\hline
\end{tabular}

\begin{tabular}{|c|c|}
\hline person selected for measurement & Male infant \\
\hline & \\
\hline & \\
\hline & \\
\hline
\end{tabular}

\begin{tabular}{|l|l|}
\hline Female adult & Male adult \\
\hline & \\
\hline & \\
\hline Pregnant__ & \\
\hline
\end{tabular}

2.0 Report information on each person selected for measurement

\begin{tabular}{|c|c|c|c|c|c|c|c|c|c|}
\hline & 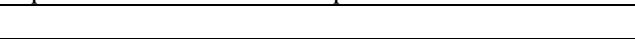 & Female infant & Male infant & Female adult & Male adult & \multirow{7}{*}{ 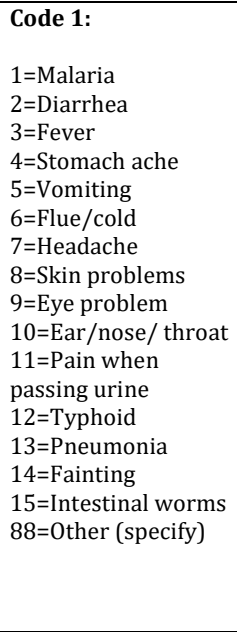 } & \multirow{7}{*}{$\begin{array}{l}\text { Code 2: } \\
\text { (If more than one, } \\
\text { record the two most } \\
\text { severe) } \\
\text { 1=Diabetes } \\
2=\text { Hypertension } \\
3=\text { Cardiovascular/ heart } \\
\text { disease } \\
4=\text { Kwashiorkor } \\
5=\text { Cancer } \\
6=\text { Rickets } \\
7=\text { If not at birth } \\
\text { (blindness) } \\
8=\text { Goiter } \\
9=\text { Gout } \\
10=\text { Bad teeth } \\
11=\text { HIV/Aids } \\
12=\text { Tuberculosis } \\
\end{array}$} & \multirow{7}{*}{$\begin{array}{l}\text { Code 3: } \\
1=\text { All of the } \\
\text { time } \\
2=\text { Most of the } \\
\text { time } \\
3=\text { Some of the } \\
\text { time } \\
4=\text { None/almos } \\
\text { t none of the } \\
\text { time }\end{array}$} & \multirow{7}{*}{$\begin{array}{l}\text { Code 4: } \\
0=\text { Never } \\
1=\text { Sometimes } \\
\text { 2=Frequently } \\
\text { 88=Not asked } \\
\text { because } \\
\text { respondent } \\
\text { may be } \\
\text { offended }\end{array}$} \\
\hline 1 & $\begin{array}{l}\text { During the last } 2 \text { weeks, did any selected HH } \\
\text { member suffer from an acute illness/condition that } \\
\text { resulted in weight loss? (Code } 1 \text { ) }\end{array}$ & & & & & & & & \\
\hline 2 & $\begin{array}{l}\text { Does this member suffer from any chronic diseases? } \\
\text { (Code 2) }\end{array}$ & & & & & & & & \\
\hline 3 & $\begin{array}{l}\text { Does this infant have a pot belly or skin/hair } \\
\text { changes? }{ }^{1}\end{array}$ & & & & & & & & \\
\hline 4 & $\begin{array}{l}\text { How often does your occupation/work activity } \\
\text { require lots of physical effort in a typical week? } \\
\text { (Code 3) }\end{array}$ & & & & & & & & \\
\hline 5 & Do you drink alcohol? (Code 4) & & & & & & & & \\
\hline 6 & $\begin{array}{l}\text { Did you ever regularly smoke cigarettes? }(0=\text { No } \\
1=\text { Yes })\end{array}$ & & & & & & & & \\
\hline
\end{tabular}




\begin{tabular}{|c|c|c|c|c|c|}
\hline \multicolumn{2}{|c|}{ 3.0 Report result of measurements } & Female infant & Male infant & Female adult & \multirow[t]{2}{*}{ Male adult } \\
\hline 1 & Calibration weight & & & & \\
\hline 2 & Weight in kg & $=\mathrm{kg}$ & $\mathrm{kg}$ & $\mathrm{kg}$ & $=\mathrm{kg}$ \\
\hline 3 & Type of cloth ( $0=$ Light clothing $1=$ Heavy clothing) & & & & \\
\hline 4 & Height in $\mathrm{cm}$ & - $\mathrm{cm}$ & $c \mathrm{~cm}$ & ccm & c $\mathrm{cm}$ \\
\hline 5 & Hip circumference in cm & & & c $\mathrm{cm}$ & c cm \\
\hline 6 & Waist circumference in $\mathrm{cm}$ & & & ccm & $\mathrm{cm}$ \\
\hline
\end{tabular}

4.1 Is any $\mathrm{HH}$ member currently pregnant?

4.2 PID: months:
( $0=$ No $1=Y e s$ ) (If yes, indicate PIDs and moths of pregnancy)

PID: months:

PID: months:

5.1 Is any member within the $\mathrm{HH}$ currently breastfeeding?

$(0=$ No $1=$ Yes $)$ [number of breastfeeding women]

6.1 In 2012 (May to August), was any HH member pregnant? $(0=$ No $1=$ Yes $)$

6.2 If yes, how many HH members? [number of pregnant women]

7.1 In 2012 (May to August), was any HH member breastfeeding? $(0=$ No $1=$ Yes $)$

7.2 If yes, how many HH members? [number of breastfeeding women] 
Smallholder participation in certification schemes / adoption of mobile phone technologies

Part 2: Gender disaggregated part (Make sure to interview the male and female respondent separately!)

Name of the respondent:

\section{SECTION 15: CHOICE EXPERIMENT}

1.1 Carefully explain the procedure using choice card No. 0 and translate the description of the pictures, record choices made by the respondent.

\begin{tabular}{|l|l|c|c|c|}
\hline & Choice set number & Indicate respondent's choice here with a 1 (put 0 for the options not chosen) \\
\hline Choice set & & & Option 2 & Option 3 \\
\hline Choice set & & & & \\
\hline Choice set & & & & \\
\hline Choice set & & & & \\
\hline Choice set & & & & \\
\hline Choice set & & & & \\
\hline
\end{tabular}

\section{SECTION 16: ATTITUDES TOWARDS CERTIFICATION}

$(0=$ No $1=Y e s) \rightarrow$ If no, move to question 3.

2.1 Do you think your HH benefits from being certified? ___ ( $0=$ No $1=$ Yes $)$

2.2 Do you feel you personally benefit from certification? ___ ( $\quad(0=$ No $1=Y e s)$

[only report the most important benefit derived from certification]

Code 1: $1=$ =Economic benefits (higher price, more income, better market access) $2=$ Training opportunities $3=$ Social benefits (bargai ning power, trust among members, collective activities, development projects, being famous) $4=0$ ther (specify)

2.4 Would you say that certification is costly for your HH (e.g. because of required investments)? $(0=$ No $1=$ Yes $)$

2.5 Do you find it difficult to meet certification requirements such as rules regarding the use of pesticides, prohibition of child and prisoner labor, or coffee quality requirements?

3.0 Only ask NON certified respondents that were NEVER certified. $\rightarrow$ If the HH is certified, move to next section.

3.1 Did you ever consider participating in a certification scheme such as UTZ, Fairtrade, Organic?

3.2 Did anyone ever offer you support to become certified (e.g. farmer organization or NGO)? $(0=$ No $1=$ Yes $)$

3.3 Do you think your $\mathrm{HH}$ could benefit from certification? $(0=$ No $1=$ Yes $)$

3.4 Do you think you could personally benefit from certification?

$(0=\mathrm{No}$

$(0=$ No $1=$ Yes $)$

3.5 If yes, what would you think would be the GREATEST benefit YOU could derive from certification? (Code 1) (only report the MOST important benefit) Code 1: $1=$ Economic benefits (higher price, more income, better market access) $2=$ Training opportunities $3=$ Social benefits (bargaining power, trust among members, collective activities, development projects) 4=0ther (specify)

3.6 Do you feel certification would be too expensive for your $\mathrm{HH}$ (because it requires certain investments)? $(0=$ No $1=Y e s)$

3.7 Would you find it difficult to meet certification requirements such as rules regarding the use of pesticides, prohibition of child and prisoner labor, or coffee quality requirements? $(0=$ No $1=$ Yes $)$ 


\section{SECTION 17: SOCIAL NETWORKS, ACCESS TO SERVICES (S.I \&S.L)}

\begin{tabular}{|c|c|}
\hline \multicolumn{2}{|c|}{ 1.0 Access to services } \\
\hline 1 & Are you a (registered) member of any group? \\
\hline 2 & Is any other HH member a member of any group? \\
\hline 3 & $\begin{array}{l}\text { Did you hold any leadership position/role in the community within } \\
\text { the last } 3 \text { years? }\end{array}$ \\
\hline 4 & $\begin{array}{l}\text { Did any other member of this HH hold any leadership position/role } \\
\text { in the community within the last } 3 \text { years? }\end{array}$ \\
\hline 5 & $\begin{array}{l}\text { Are you able to receive credit for agricultural production when you } \\
\text { need such credit? }\end{array}$ \\
\hline 6 & $\begin{array}{l}\text { Has anyone in your HH in fact taken any loan or borrowed cash/in- } \\
\text { kind in the past } 12 \text { months? }\end{array}$ \\
\hline 7 & $\begin{array}{l}\text { Who makes the decision about what to do with the borrowed } \\
\text { money/item? (Code 4) }\end{array}$ \\
\hline 8 & Do you, personally, (not your HH) have a savings account? \\
\hline 9 & Did you have a savings account in $2012 ?$ \\
\hline 10 & Does any other HH member have a savings account? \\
\hline
\end{tabular}

\begin{tabular}{|l|l}
$0=$ No $1=Y e s$ \\
\hline
\end{tabular}

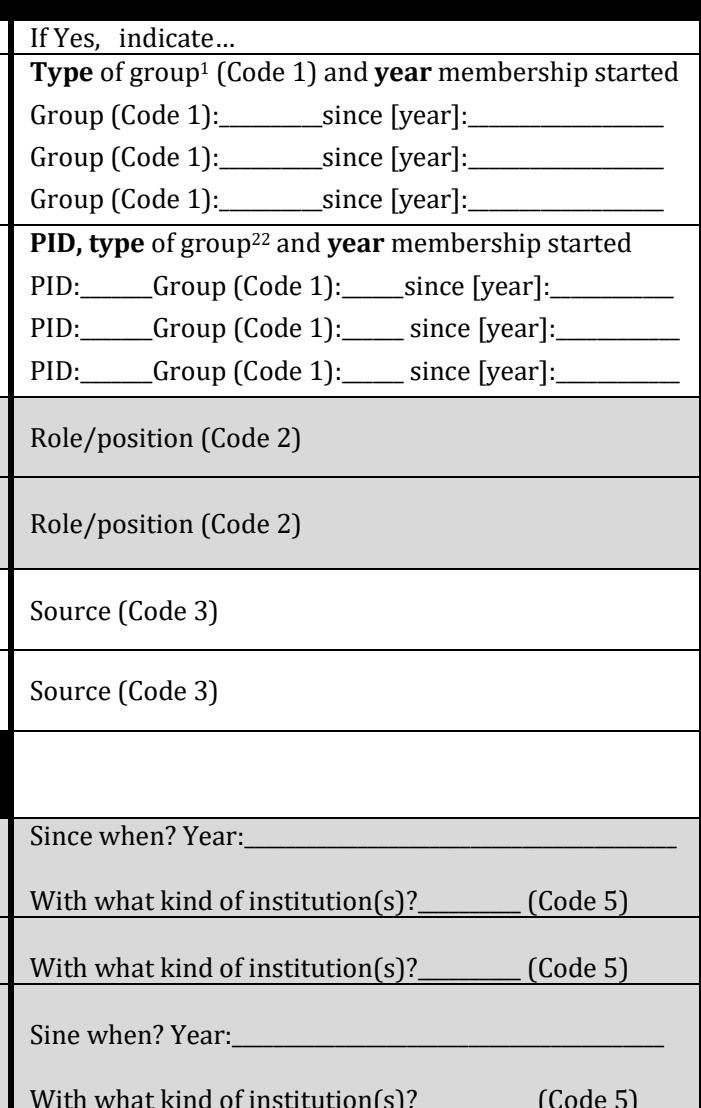

Codes

Code 1

$1=$ Farmer organization

$2=$ Women's group

$3=$ Credit and savings group

4=Religious groups

ce groups (e.g. burial societies)

$6=$ Trade and business associations

\section{Code 2: $\quad 4=$ Religious organization}

$1=$ Sub-county chief $\quad 5=$ Farmer organization/group

$2=$ Parish chief $\quad 6=$ Extension officer

$\begin{array}{ll}3=\text { Local Council } & 7=\text { Cultural-spiritual leader } \\ \text { Committee } & \text { 8=Lead farmer }\end{array}$

Code 3:

=Farmer group $\quad 6=$ Local agro dealers

$2=$ Cooperative $\quad 7$ =Farmers

4=Local bank $\quad 8=$ Mobile money service providers

$5=$ Exporter $\quad 88=0$ ther (specify)

Code 4: $\quad 3=$ Jointly

$\begin{array}{ll}\text { Code 4: } & \text { 3=Jointly } \\ 1=\text { HHD } & \text { 88=0ther (specify) }\end{array}$

$2=$ Spouse

$=$ Formal bank (e.g. Centenary)

=Semi-formal financial institution (e.g. MFI, SACCO)

=Mobile Telephone Operator

$4=0$ ther (specify)

5.0 Agricultural training and information

5.1 Are you able to receive agricultural information when you need such information? $(0=$ No $1=$ Yes $)$

5.2 If yes, what is the most important source (Code 5) (allow multiple answers, but START with the most important)

Code 5: 1=Extension staff 2 =Extension bulletins $4=0$ ther farmers $5=$ Radio $\quad 6=$ TV $\quad 7=$ Newspaper $8=$ Mobile phone alerts $88=0$ ther (specify)

${ }_{1}^{1}$ If multiple, indicate 3 most important ones. 


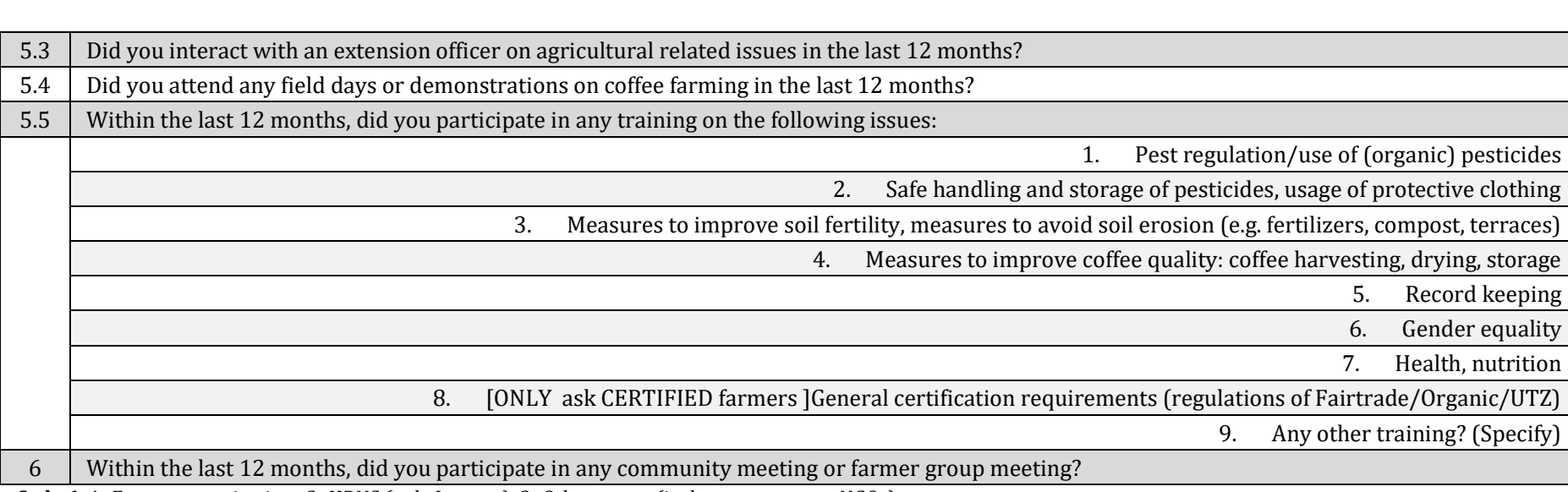

\begin{tabular}{|l|l|l|l|}
\hline $0=$ No 1=Yes & No. of times & Provider (Code 6) \\
\hline & & \\
\hline & & & \\
\hline & & & \\
\hline & & & \\
\hline & & & \\
\hline & & & \\
\hline & & & \\
\hline
\end{tabular}
Code 6: $1=$ Farmer organization $2=\mathrm{HRNS}$ (only Luwero) $3=0$ ther actors (incl. government or NGOs)

7.0 Speaking in the public

1 Do you feel comfortable speaking up during community or farmer group/organization meetings to share your point of view?

2 Do you feel comfortable speaking up in public to protest the misbehavior of authorities or elected officials?

\begin{tabular}{|l|}
\hline (Code 7) \\
\hline
\end{tabular}

\section{Code 7:}

$1=\mathrm{No}$, not at all

$2=$ Yes, but with a great deal of difficulty

$3=$ Yes, but with little difficulty

$4=$ Yes, fairly comfortable
$5=$ Yes, very comfortable

\begin{tabular}{|c|c|c|c|c|c|}
\hline \multicolumn{2}{|r|}{ 8.0 How are travel arrangements in your HH? Do you travel? } & Yes, alone & Yes, accompanied by...(Code 8) & No/never & Distance (min travel time) \\
\hline 1 & Do you travel to markets? & & & & $\min$ \\
\hline 2 & Do you travel to relatives? & & & & $\min$ \\
\hline 3 & Do you travel to health centers/doctor? & & & & $\min$ \\
\hline 4 & Do you travel to Masaka/Luwero town? & & & & $\min$ \\
\hline 5 & Do you travel to Kampala? & & & & $\min$ \\
\hline
\end{tabular}

Code 8: 1=By HHD 2=By male HH member 3=By female HH member $\quad 88=0$ ther (specify)

SECTION 18: INTRA HOUSEHOLD DECISION MAKING

\section{Do you agree with the following statements?}

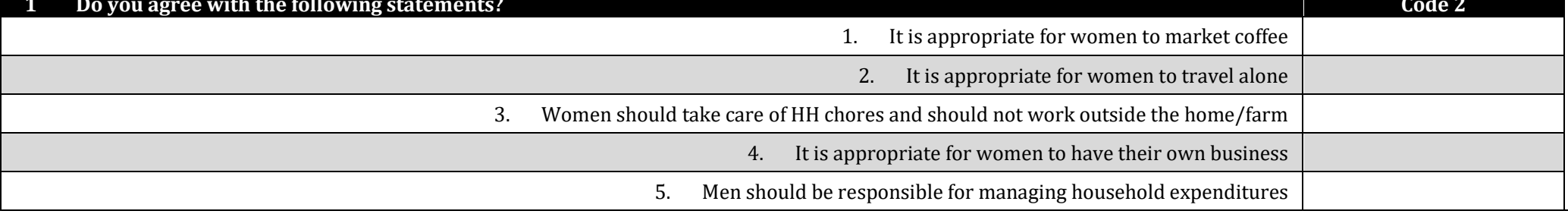

Code 2:

3. Women should take care of HH chores and should not work outside the home/farm

$1=$ Strongly agree

$2=$ Agree

$3=$ Disagree
$4=$ Strongly disagree 


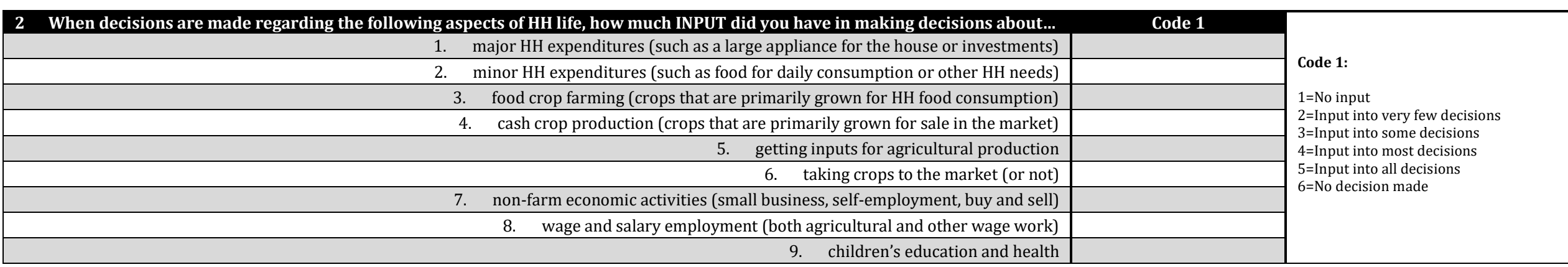

\section{SECTION 19: TIME ALLOCATION}

1 In the last complete 24 hours, starting yesterday morning at 3 am, finishing 2:59 am of the current day, which activities did you carry out? (Intervals are marked in 15 min intervals. Please insert numbers given under code 1 . Ask respondents to narrate their day themselves.)

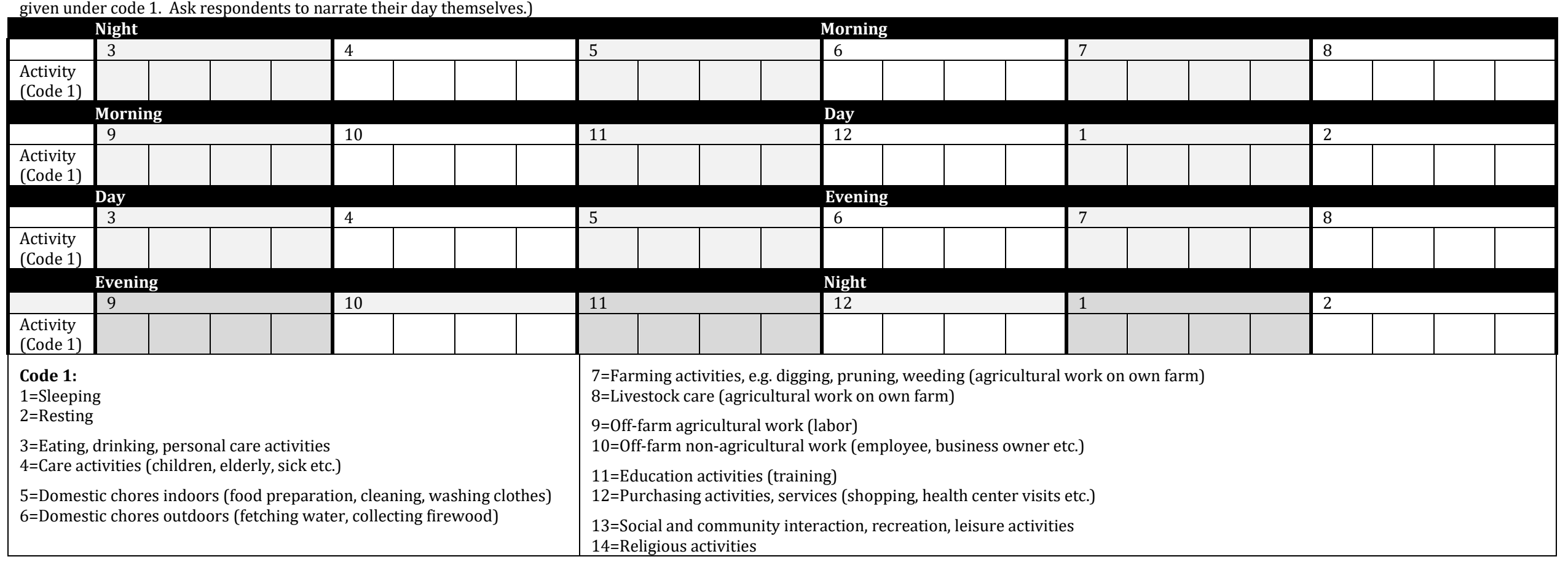

2 How satisfied are you with your available time for leisure activities like visiting neighbors, watching TV, listening to the radio, seeing movies or doing sports?(Code 2 ) Code 2: 1=Very satisfied $2=$ Satisfied $3=$ =Fairly satisfied $\quad 4=$ Unsatisfied $\quad 5=$ Very unsatisfied 
Part 2: Gender disaggregated part (Make sure to interview the male and female respondent separately!)

Name of the respondent:

\section{SECTION 15: CHOICE EXPERIMENT}

1.1 Carefully explain the procedure using choice card No. 0 and translate the description of the pictures, Record choices made by the respondent.

\begin{tabular}{|l|l|c|c|c|}
\hline & & \multicolumn{2}{|c|}{ Indicate respondent's choice here with a 1 (put 0 for the options not chosen) } \\
\hline & Choice set number & Option 1 & Option 2 & Option 3 \\
\hline Choice set & & & & \\
\hline Choice set & & & & \\
\hline Choice set & & & & \\
\hline Choice set & & & & \\
\hline Choice set & & & & \\
\hline Choice set & & & & \\
\hline
\end{tabular}

\section{SECTION 16: ATTITUDES TOWARDS CERTIFICATION}

2.0 Is or was this HH ever certified?

2.1 Do you think your HH benefits from being certified?

2.2 Do you feel you personally benefit from certification?

$(0=\mathrm{No} \quad 1=\mathrm{Yes}) \rightarrow$ If no, move to question 3. $(0=$ No $1=Y e s)$

2.3 If yes, what is the GREATEST benefit for YOU? (Code 1) $(0=$ No $1=$ Yes)

Code 1: 1 =Economic benefits (higher price, more income, better market access) $2=$ Training opportunities $3=$ Social benefits (bargaining power, trust among members, collective activities, developmen projects, being famous) $4=0$ ther (specify)

2.4 Would you say that certification is costly for your HH (e.g. because of required investments)? $(0=$ No $1=$ Yes)

2.5 Do you find it difficult to meet certification requirements such as rules regarding the use of pesticides, prohibition of child and prisoner labor, or coffee quality requirements?

3.0 Only ask NON certified respondents that were NEVER certified. $\rightarrow$ If the HH is certified, move to next section

3.1 Did you ever consider participating in a certification scheme such as UTZ, Fairtrade, Organic?

3.2 Did anyone ever offer you support to become certified (e.g. farmer organization or NGO)? $(0=$ No $1=$ Yes)

3.3 Do you think your $\mathrm{HH}$ could benefit from certification? $(0=$ No $1=$ Yes $)$

3.3 Do you think your $\mathrm{HH}$ could benefit from certification?

3.5 If yes, what would you think would be the GREATEST benefit YOU could derive from certification? (Code 1) (only report the MOST important benefit) Code 1: 1=Economic benefits (higher price, more income, better market access) $2=$ Training opportunities $3=$ Social benefits (bargaining power, trust among members, collective activities, development projects) $4=0$ ther (specify)

3.6 Do you feel certification would be too expensive for your $\mathrm{HH}$ (because it requires certain investments)? $(0=$ No $1=$ Yes $)$

3.7 Would you find it difficult to meet certification requirements such as rules regarding the use of pesticides, prohibition of child and prisoner labor, or coffee quality requirements? $(0=$ No $1=$ Yes $)$ 


\section{SECTION 17: SOCIAL NETWORKS, ACCESS TO SERVICES (S.I \&S.L)}

\begin{tabular}{|c|c|}
\hline 1 & Are you a (registered) member of any group? \\
\hline 2 & Is any other HH member a member of any group? \\
\hline 3 & $\begin{array}{l}\text { Did you hold any leadership position/role in the community within } \\
\text { the last } 3 \text { years? }\end{array}$ \\
\hline 4 & $\begin{array}{l}\text { Did any other member of this HH hold any leadership position/role } \\
\text { in the community within the last } 3 \text { years? }\end{array}$ \\
\hline 5 & $\begin{array}{l}\text { Are you able to receive credit for agricultural production when you } \\
\text { need such credit? }\end{array}$ \\
\hline 6 & $\begin{array}{l}\text { Has anyone in your HH in fact taken any loan or borrowed cash/in- } \\
\text { kind in the past } 12 \text { months? }\end{array}$ \\
\hline 7 & $\begin{array}{l}\text { Who makes the decision about what to do with the borrowed } \\
\text { money/item? (Code 4) }\end{array}$ \\
\hline 8 & Do you, personally, (not your HH) have a savings account? \\
\hline 9 & Did you have a savings account in $2012 ?$ \\
\hline 10 & Does any other HH member have a savings account? \\
\hline
\end{tabular}

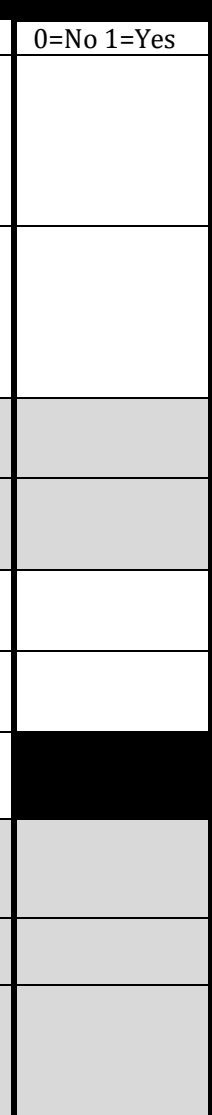

If Yes, indicate...
Type of group ${ }^{1}$ (Code 1) and year membership started
Group (Code 1):___ since [year]:___
Group (Code 1):___ since [year]:___
Group (Code 1):___since [year]:__

PID, type of group ${ }^{22}$ and year membership started

PID:___ Group (Code 1):___ since [year]:

PID:_ Group (Code 1):___ since [year] PID:___ Group (Code 1):____ since [year]

Role/position (Code 2)

Role/position (Code 2)

Source (Code 3)

Source (Code 3)

Sine when? Year:___
With what kind of institution(s)?_____ (Code 5)
With what kind of institution(s)?_____ (Code 5)
Sine when? Year:___

Codes

$1=$ Farmer organization

$2=$ Women's group

$3=$ Credit and savings group

4=-Religious groups

nce groups (e.g. burial societies)

$6=$ Trade and business associations

Other (specify)

With what kind of institution(s)?

5.0 Agricultural training and information

5.1 Are you able to receive agricultural information when you need such information? $(0=$ No $1=$ Yes $)$

5.2 If yes, what is the most important source (Code 5) (allow multiple answers, but START with the most important) Code 5: 1=Extension staff $\quad 2=$ Extension bulletins $\quad 4=0$ ther farmers $\quad 5=$ Radio $\quad 6=\mathrm{TV} \quad 7=$ Newspaper $\quad 8=$ Mobile phone alerts $\quad 88=0$ ther (specify)

1 If multiple, indicate 3 most important ones. 
\begin{tabular}{|c|l}
5.3 & Did you interact with an extension officer on agricultural related issues in the last 12 months?
\end{tabular}

5.4 Did you attend any field days or demonstrations on coffee farming in the last 12 months?

5.5 Within the last 12 months, did you participate in any training on the following issues:

1. Pest regulation/use of (organic) pesticides \begin{tabular}{|rr}
\hline & Safe handling and storage of pesticides, usage of protective clothing \\
\hline $3 . \quad$ Measures to improve soil fertility, measures to avoid soil erosion (e.g. fertilizers, compost, terraces) \\
\hline & $4 . \quad$ Measures to improve coffee quality: coffee harvesting, drying, storage \\
\hline & $5 . \quad$ Record keeping \\
\hline & $6 . \quad$ Gender equality \\
\hline & $7 . \quad$ Health, nutrition \\
\hline
\end{tabular} \begin{tabular}{|rr}
\hline & Safe handling and storage of pesticides, usage of protective clothing \\
\hline $3 . \quad$ Measures to improve soil fertility, measures to avoid soil erosion (e.g. fertilizers, compost, terraces) \\
\hline & $4 . \quad$ Measures to improve coffee quality: coffee harvesting, drying, storage \\
\hline & $5 . \quad$ Record keeping \\
\hline & $6 . \quad$ Gender equality \\
\hline & $7 . \quad$ Health, nutrition \\
\hline
\end{tabular} \begin{tabular}{|rr}
\hline & Safe handling and storage of pesticides, usage of protective clothing \\
\hline $3 . \quad$ Measures to improve soil fertility, measures to avoid soil erosion (e.g. fertilizers, compost, terraces) \\
\hline & $4 . \quad$ Measures to improve coffee quality: coffee harvesting, drying, storage \\
\hline & $5 . \quad$ Record keeping \\
\hline & $6 . \quad$ Gender equality \\
\hline & $7 . \quad$ Health, nutrition \\
\hline
\end{tabular} 8. [ONLY ask CERTIFIED farmers ] General certification requirements (regulations of Fairtrade/Organic/UTZ) Within the last 12 months, did you participate in any community meeting or farmer group meeting? 9. Any other training? (Specify) Code 6: 1=Farmer organization $2=$ HRNS (only Luwero) $3=0$ ther actors (incl. government or NGOs)

7.0 Speaking in the public

$1 \quad$ Do you feel comfortable speaking up during community or farmer group/organization meetings to share your point of view?

2 Do you feel comfortable speaking up in public to protest the misbehavior of authorities or elected officials?

\begin{tabular}{|l|l|l|l|}
\hline & $0=$ No $1=$ Yes & No. of times & Provider (Code 6) \\
\hline & & & \\
\hline & & & \\
\hline & & & \\
\hline & & & \\
\hline & & & \\
\hline & & & \\
\hline & & & \\
\hline & & & \\
\hline
\end{tabular}

8.0 How are travel arrangements in your HH? Do you travel?

\begin{tabular}{c|c|} 
8.0 & How are travel arrangements \\
\hline 1 & Do you travel to markets?
\end{tabular}

2 Do you travel to relatives?

3 Do you travel to health centers/doctor?

4 Do you travel to Masaka/Luwero town?

$\begin{array}{ll}5 & \text { Do you travel to Kampala? }\end{array}$

Code 8: 1=By HHD $\quad$ 2=By male HH member $3=$ =By female HH member $\quad$ 88=0ther (specify)

\section{SECTION 18: INTRA HOUSEHOLD DECISION MAKING}

\section{$1 \quad$ Do you agree with the following statements?}

\begin{tabular}{|l|l|l|r|}
\hline Yes, alone & Yes, accompanied by...(Code 8) & No/never & Distance (min travel time) \\
\hline & & & $\min$ \\
\hline & & & $\min$ \\
\hline & & & $\min$ \\
\hline & & & $\min$ \\
\hline
\end{tabular}

1. It is appropriate for women to market coffee 2. It is appropriate for women to travel alone

3. Women should take care of HH chores and should not work outside the home/farm 4. It is appropriate for women to have their own business
Code 2

Code 2:

$1=$ Strongly agree

$2=$ Agree
$3=$ Disagree

$4=$ Strongly disagree 


\begin{tabular}{|c|c|c|c|}
\hline 2 & When decisions are made regarding the following aspects of HH life, how much INPUT did you have in making decisions about... & Code 1 & \multirow{10}{*}{$\begin{array}{l}\text { Code 1: } \\
\text { 1=No input } \\
2=\text { Input into very few decisions } \\
3=\text { Input into some decisions } \\
4=\text { Input into most decisions } \\
5=\text { Input into all decisions } \\
6=\text { No decision made }\end{array}$} \\
\hline & 1. major HH expenditures (such as a large appliance for the house or investments) & & \\
\hline & 2. minor HH expenditures (such as food for daily consumption or other HH needs) & & \\
\hline & 3. food crop farming (crops that are primarily grown for $\mathrm{HH}$ food consumption) & & \\
\hline & 4. cash crop production (crops that are primarily grown for sale in the market) & & \\
\hline & 5. getting inputs for agricultural production & & \\
\hline & 6. $\quad$ taking crops to the market (or not) & & \\
\hline & 7. non-farm economic activities (small business, self-employment, buy and sell) & & \\
\hline & 8. wage and salary employment (both agricultural and other wage work) & & \\
\hline & 9. children's education and health & & \\
\hline
\end{tabular}

\section{SECTION 19: TIME ALLOCATION}

1 In the last complete 24 hours, starting yesterday morning at $3 \mathrm{am}$, finishing 2:59 am of the current day, which activities did you carry out? (Intervals are marked in 15 min intervals. Please insert numbers given under code 1 . Ask respondents to narrate their day themselves.)

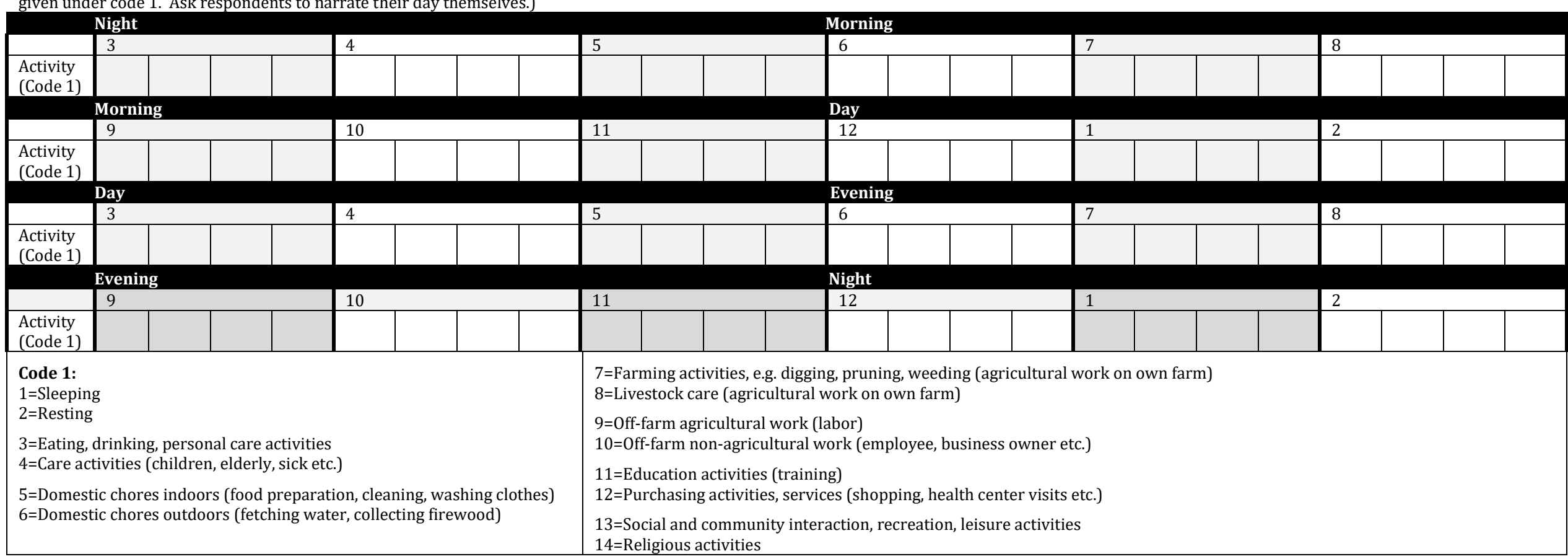

2 How satisfied are you with your available time for leisure activities like visiting neighbors, watching TV, listening to the radio, seeing movies or doing sports?(Code 2 ) Code 2: 1=Very satisfied $\quad 2=$ Satisfied $3=$ =Fairly satisfied $\quad 4=$ =Unsatisfied $\quad 5=$ Very unsatisfied 